

\section{Propositions}

1. The economic burden of rabies in cattle is an under-utilized incentive for canine vaccination.

(this thesis)

2. Maximising canine vaccination coverage does not maximise net health benefit. (this thesis)

3. Multi-disciplinary research requires systems thinking.

4. Even when the utility of two options is similar, the option chosen still matters.

5. In the current era, an increasing trend of intercultural diversities and controversial thoughts is inevitable.

6. Education does not, per definition, make the world a better place to live.

Propositions belonging to the thesis, entitled

'Rabies in Ethiopia: Modelling the burden and the effectiveness of control'

Tariku Jibat Beyene

Wageningen, 18 December 2017 
Rabies in Ethiopia: Modelling the burden and the effectiveness of control

Tariku Jibat Beyene 


\section{Thesis committee}

\section{Promotor}

Prof. Dr H. Hogeveen

Personal chair at the Business Economics Group

Wageningen University \& Research

\section{Co-promotor}

Dr M.C.M. Mourits

Associate professor, Business Economics Group

Wageningen University \& Research

\section{Other members}

Prof. Dr J. Zinsstag, Swiss Tropical and Public Health Institute, Basel, Switzerland

Prof. Dr M.A. Koelen, Wageningen University \& Research

Prof. Dr M.C.M. de Jong, Wageningen University \& Research

Dr M.A.P.M. van Asseldonk, Wageningen University \& Research

This research was conducted under the auspices of the Wageningen School of Social Sciences (WASS). 


\title{
Rabies in Ethiopia: Modelling the burden and the effectiveness of control
}

\author{
Tariku Jibat Beyene
}

\section{Thesis}

submitted in fulfillment of the requirements for the degree of doctor

at Wageningen University

by the authority of the Rector Magnificus,

Prof. Dr A.P.J. Mol,

in the presence of the

Thesis committee appointed by the Academic Board

to be defended in public

on Monday 18 December 2017

at $1: 30$ p.m. in the Aula. 
Tariku Jibat Beyene

Rabies in Ethiopia: Modelling the burden and the effectiveness of control, 194 pages.

$\mathrm{PhD}$ thesis, Wageningen University, Wageningen, the Netherlands (2017) With references, with summary in English

DOI: $10.18174 / 424984$

ISBN: 978-94-6343-217-7 
To Asni, Fikru and Jibat 



\title{
Table of Contents
}

\author{
Abstract \\ Chapter 1 General introduction 11 \\ Chapter 2 Systematic review and meta-analysis on dog rabies vaccination $\quad 19$ \\ coverage in Africa: A question of dog accessibility or cost recovery? \\ Chapter 3 Dog rabies data reported to multinational organizations from 39 \\ Southern and Eastern African Countries \\ Chapter 4 Incidence and economic impact of rabies in the cattle population of 49 \\ Ethiopia \\ Chapter 5 Estimating the burden of rabies in Ethiopia by dog bite victims $\quad 73$ \\ tracing \\ Chapter 6 Determinants of health seeking behaviour following rabies exposure \\ 97 \\ in Ethiopia \\ Chapter 7 Evaluation of canine vaccination strategies to control rabies in 119 \\ Ethiopia \\ $\begin{array}{lll}\text { Chapter } 8 \text { General discussion } & 165\end{array}$ \\ Summary 183 \\ Acknowledgments 189

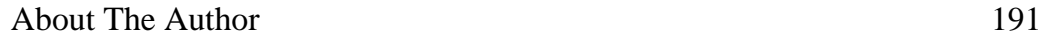 \\ Completed Training and Supervision Plan 193
}





\section{Abstract}

Rabies claims the lives of more than 24,000 people in Africa annually, but efforts to control the disease are still lacking, particularly in sub-Saharan Africa such as Ethiopia. The overall objective of this study was to support the design of an appropriate cost-effective rabies control policy in Ethiopia by providing insights in the health burden of the disease and its economic impacts, as well as an understanding of the relationship between intervention levels, implementation costs and potential returns.

As most human rabies cases result from the bite of domestic dogs, the disease can be eliminated by mass canine rabies vaccination. An extensive literature review on mass canine vaccination programs in Africa indicated that most dogs in Africa are owned and therefore accessible for vaccination, but vaccination coverages strongly depend on the implemented cost schemes. Canine vaccination in Ethiopia is voluntarily based, i.e. “owner-charged”, resulting in one of the lowest coverages in the world. To assess the current burden of rabies in Ethiopia a retrospective study was conducted by collecting data on human rabies exposure over the period of one year through extensive bite case searching in three representative districts of Ethiopia. Extrapolation of the results to national level indicated an annual average of 3,000 human deaths and 97,000 rabies-exposed persons treated at an average costs of 21 USD per case, causing 2 million USD on treatment costs per year and a health loss of about 93,000 DALYs. About $77 \%$ of the exposure cases visited a health centre, while only $57 \%$ received sufficient doses of post exposure treatment. Important factors that influenced victim's medical treatment seeking behaviour were ownership status of the biting dog, severity of the bite, body part bitten, monthly spending and distance to the nearest health centre whereas the likelihood of receiving sufficient doses of treatment were determined by monthly spending and distance to health centre. The district in which victims lived appeared to have a relevant influence on the likelihood of seeking medical treatment but not on the likelihood of treatment compliance. By means of a structured questionnaire administered to cattle-owning households the economic impact of rabies in livestock was assessed. Herd-level incidence rates appeared higher in the mixed crop-livestock system (21\%) than in the pastoral system (11\%). Average economic losses per herd due to rabies were estimated at 49 USD per year for the mixed-crop livestock system, and at 52 USD per year for the pastoral system.

In light of policy support for rabies control, an evaluation of the cost-effectiveness of control strategies was performed by the use of a dynamic epidemiological model coupled with an economic analysis to predict the human health impact and economic benefit (reducing human treatment costs and livestock rabies-related losses) across a range of vaccination scenarios. Human exposures, human deaths, and rabies-related livestock losses decreased monotonically with increasing vaccination coverage. In the evaluated urban and rural districts, $50 \%$ coverage was identified as most likely scenario to provide the greatest net health benefits at the WHO-recommended willingness-to-pay threshold over a time frame of 10 years. The additional economic benefit from rabies control in livestock justified the additional costs of vaccination campaigns with higher coverages than would have been efficient from a strict human health perspective, highlighting the importance of applying a broad perspective with regard to the evaluation of vaccination benefits.

Key words: Rabies, Dogs, Cattle, Cost-effectiveness, Ethiopia, Health burden, Health economics, Medical treatment seeking behavior, One health, Post-exposure treatment, Vaccination, Zoonoses 

Chapter 1

General Introduction 


\subsection{Background}

Rabies is among the oldest and most recognised diseases affecting both humans and animals (Jackson and Wunner, 2013; King et al., 2004). Rabies in humans has one of the highest case fatalities of all infectious diseases. Every year, rabies is responsible worldwide for an estimated 60,000 human deaths, 15 million people receive rabies post-exposure prophylaxis (PEP), 4 million disability-adjusted life years (DALYs) and 9 billion USD economic losses. The economic burden is caused, by premature death, direct costs of post-exposure prophylaxis and lost income whilst seeking PEP, costs to the veterinary sector due to dog vaccination, and losses due to livestock deaths (Hampson et al., 2015). More than 95\% of the human deaths and related DALYs occur in Africa and Asia, while it has been eliminated from human population in North America, Europe, Australia, and most parts of South America (Davlin and VonVille, 2012; Vigilato, et al., 2012). The livestock-related loss is mainly in African countries with livestockdependent economies, especially Ethiopia, Sudan, and Tanzania (Hampson et al., 2015).

The principal manner of transmission to humans is through an animal bite in which the virus in saliva is inoculated. Dogs are identified as the major reservoir. The virus is highly neurotropic, and once it enters the body through a break in the skin or mucous membrane, it migrates along the nerves from the site of infection to the brain where it causes fatal encephalitis. From the brain, the virus travels back to the organs of the body, eventually causing them to shut down. Symptoms of the disease are variable, but some distinctive signs include severe anxiety, fear of water, and fear of air (Jackson and Wunner, 2013).

Dog vaccination is indicated as the most cost-effective prevention method (OIE, 2011). In humans it can also be prevented by appropriate post-exposure prophylaxis treatment. However, treatment is not always available and affordable in resource-limited countries (Zinsstag et al., 2009). In Sub-Saharan Africa, rabies' control is rarely prioritised, due to poor quality of information on its distribution and mortality, and morbidity rates, as well as to a lack of insight in its related economic losses.

\subsection{Problem statement}

Rabies has been recognised and documented in Ethiopia as early as the $17^{\text {th }}$ century (Fekadu, 1982). The first epidemic was recorded in Addis Ababa in 1903 (Pankhurst, 1970). The Ethiopian Institute of Public Health and several others studies have reported occurrence of rabies in several regions of the country (Yimer et al., 2002; EHNRI, 2012; Jemberu et al., 2013; G/hiwot et al., 2016; Tschopp et al., 2016; Teklu et al., 2017). Since 2007, an annual estimate of 12 exposed human cases and 1.6 deaths per 100,000 population has been reported (Deressa et al., 2013). These numbers do not represent countrywide incidences, but mainly reflect on incidences specific for the area geographically close to the capital city of Ethiopia (EHNRI, 
2012). The true burden of the disease is thought to be higher, as a considerable proportion of animal-bite victims might not report to health centres, especially in many African rural communities (Cleaveland et al., 2002; Tschopp et al., 2016). Over 98\% of the human exposure and deaths cases were caused by a bite from unvaccinated domestic dogs (Yimer et al., 2012; Jemberu et al., 2013).

Ethiopia is a land-locked country in the Horn of Africa with a surface area of 1.1 million km² (FAO, 2017). About 80\% of Ethiopians are dependent on agriculture, mostly living in rural areas (Leta and Mesele, 2014). Rural areas are heavily reliant on livestock for household revenues and cattle serves as traction power for crop land cultivation (Behnke and Metaferia, 2011). With 57 million heads of cattle, Ethiopia owns the largest cattle population in Africa (CSA, 2012). As the livestock sector in Ethiopia provides livelihoods to $65 \%$ of the human population (Solomon, 2003), occurrence of rabies in cattle can have serious economic consequences. Accurate information on the incidences of rabies in humans, dogs and livestock is, however, scarce in Ethiopia (Jemberu et al, 2013), due to the absence of proper registration systems. Rabies-related medical and veterinary records are incomplete and cannot be used for a proper quantification of the impact of rabies (Tefera et al., 2002; Adane et al., 2013; EHNRI, 2012).

The canine vaccination coverage in Ethiopia is far lower than the standard WHO recommendation of $70 \%$ vaccination coverage (Coleman and Dye, 1996). The dog vaccination coverage in cities is far below this recommended coverage (Ali et al., 2010) and even nonexistent in rural communities (Abay et al., 2011). The coverage is limited due to the lack of knowledge on rabies epidemiological distribution, lack of insight into the actual health and economic burden of rabies which might also prevent governments and stakeholders from allocating appropriate resources for control and prevention (Nel, 2013). Moreover, dog vaccination services are generally concentrated in the cities and only dog-owners who can afford the vaccination costs will consider the option to vaccinate.

During the last decade, there has been an increasing interest to launch a national rabies control programme to mitigate the impact of the disease on human and animal health as well as to minimise the economic burden (Coetzer et al., 2016; Pieracci et al., 2016). Designing an appropriate cost-effective control policy requires a clear understanding of the disease burden, the economic impacts, the factors associated with the burden, understanding the relationship between intervention levels, the costs of intervention, and their respective potential returns. Thus, generating empirical data on the burden of rabies in terms of public health, treatment costs, and livestock-related losses, and evaluation of cost-effective control options are of vital significance to support decision making on control (WHO, 2017). In addition, considering a varying human population and canine densities, and livestock production systems, evaluation 
of the cost-effectiveness on the level of vaccination coverage is relevant to support prioritisation under such a resource-constrained situation.

\subsection{Objectives}

The overall objective of this thesis was to support decision-making in the control of rabies in Ethiopia by providing evidence on its health and economic burden in humans as well as cattle, and evaluate the cost-effectiveness of canine vaccination strategies in different human settlements and cattle production systems. The broader objective was addressed through the following six sub-objectives:

1. To systematically assess the level of canine vaccination coverage against rabies in Africa.

2. To examine the trend of rabies incidences in African countries based on data reported to regional and international organisations through human and veterinary health authorities.

3. To estimate the incidence and economic impacts of cattle rabies in two representative livestock production systems of Ethiopia.

4. To estimate the public health burden and post-exposure treatment costs due to rabies in three representative human settlement settings of Ethiopia.

5. To assess factors affecting likelihood of medical treatment-seeking and compliance to treatment regimen behaviour following rabies exposure

6. To evaluate canine vaccination strategies to control rabies in Ethiopia.

\subsection{Thesis outline}

This thesis is organised into eight chapters, including this general introduction (as Chapter 1) and a general discussion (as Chapter 8). Chapters 2 to 7 address each of the aforementioned sub-objectives. Figure 1 provides a schematic overview of the structure of the thesis. Chapter 1 provides a brief account on global and Ethiopian context of rabies burden and its level of control, the motivation of this study, objectives, and outline of this thesis. In order to obtain insight in dog rabies vaccination coverage in Africa and dog rabies data reported from human health and veterinary authorities to World Organisation for Animal health (OIE) and Southern and Eastern African Rabies Group (SEARG), a systematic literature review (Chapter 2) and database exploration (Chapter 3) were carried out. Rabies incidences, health burden and associated economic losses in humans and cattle are assessed in Chapters 4 and 5. Chapter 6 is dedicated to factors influencing rabies-exposed people's medical treatment-seeking behaviour in terms of visiting health centres and receiving sufficient doses of post-exposure prophylaxis. Based on the empirical evidence and information generated in the preceding chapters, an overarching chapter, Chapter 7 evaluates different canine vaccination coverages in urban and rural settings to identify the cost-effective vaccination strategy. Finally, the concluding chapter (8) synthesises the main findings of this thesis, explains the methods applied, outlines the policy implication of the results for rabies control and outlines future research directions. 


\section{Chapter 1}

General Introduction

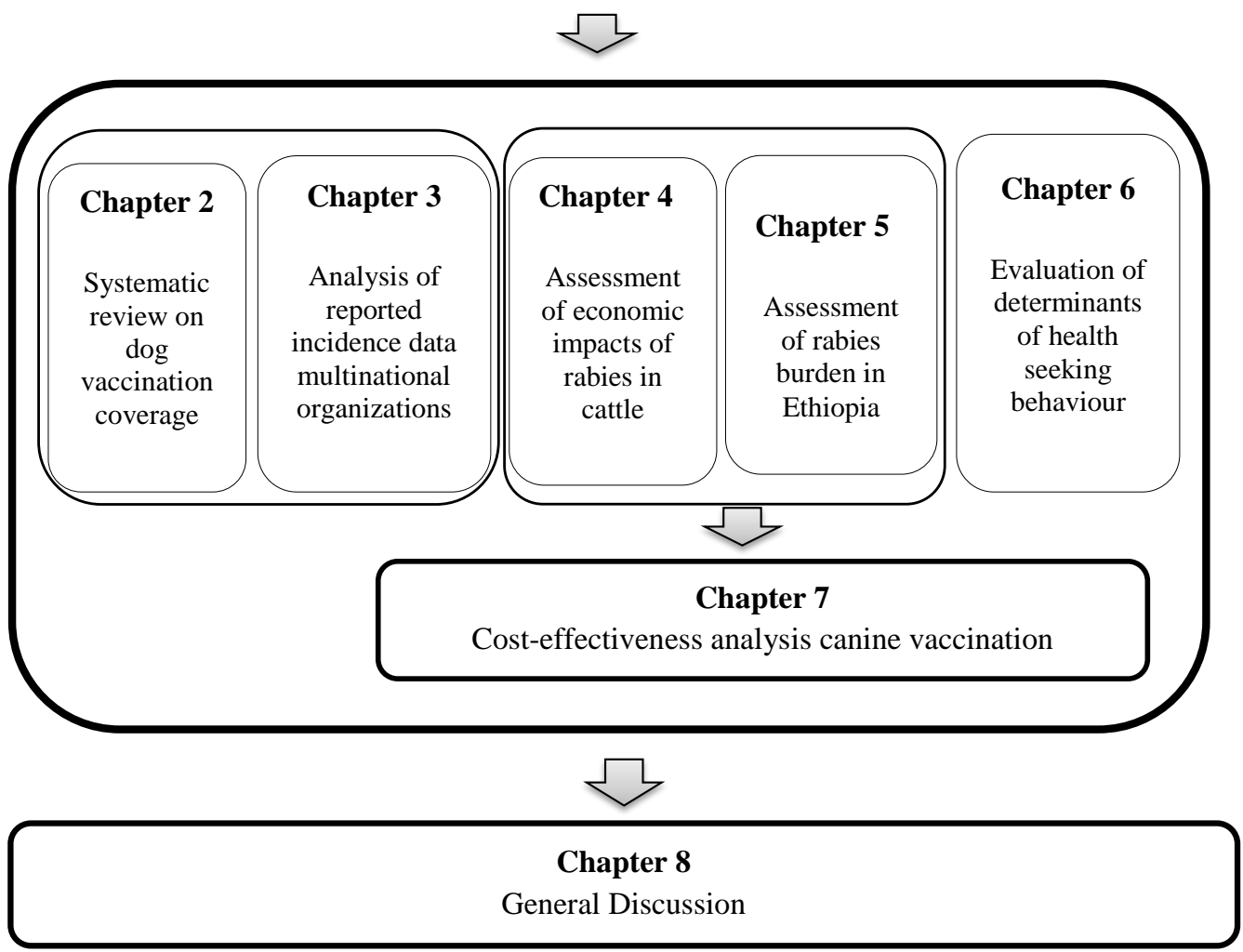

Figure 1.1. Schematic diagram showing linkage between components of the study 


\subsection{References}

Abay, G. Y., Bauer, H., Gebrihiwot, K.,and Deckers, J., 2011. Peri-urban spotted hyena (Crocuta crocuta) in northern Ethiopia: diet, economic impact, and abundance. Euro Jour of Wildlife Res, 57(4), 759-765.

Adane, K., Muluye, D., Abebe, M., 2013. Processing medical data: a systematic review. Archives of Public Health, 71(1), 27.

Ali, A., Yimer E., Mengistu F., Hussen K., and K, T., 2010. Overview of Rabies in and around Addis Ababa, in Animals Examined in EHNRI Zoonoses Laboratory Between, 2003 and 2009. Ethio Vet J, 14(2), 91-101.

Behnke, R.,and Metaferia, F., 2011. The contribution of livestock to the Ethiopian economyPart II. IGAD LPI Working Paper No. 02 - 11, Addis Ababa, Ethiopia.

Coetzer, A., Kidane, A. H., Bekele, M., Hundera, A. D., Pieracci, E. G., Shiferaw, M. L., . . . Nel, L. H. 2016. The SARE tool for rabies control: Current experience in Ethiopia. Antiviral Research, 135, 74-80. doi:10.1016/j.antiviral.2016.09.011.

Cleaveland, S., Fevre, E. M., Kaare, M., \& Coleman, P. G. 2002. Estimating human rabies mortality in the United Republic of Tanzania from dog bite injuries. Bulletin of the World Health Organization, 80(4), 304-310.

Coleman, P. G.,and Dye, C., 1996. Immunization coverage required to prevent outbreaks of dog rabies. Vaccine, 14(3), 185-186.

CSA (Central Statistical Agency), 2012. Household Consumption and Expenditure (HCE) Survey 2010/11. The Federal Democratic Republic of Ethiopia Central Statistical Agency. Analytical report. Addis Ababa, Ethiopia. pp. 47-69.

Davlin, S. L.,and VonVille, H. M., 2012. Canine rabies vaccination and domestic dog population characteristics in the developing world: a systematic review. Vaccine, 30(24), 3492-3502.

EHNRI, 2012. The Ethiopian Health and Nutrition Research Institute. Proceedings of The National Workshop on Rabies Prevention and Control in Ethiopia. October 18-19, 2012. Adama, Ethiopia.

FAO, 2017. http://www.fao.org/ag/aquastat/ETHIOPIA.HTM. access date May 22, 2017.

Fekadu, M., 1982. Rabies in Ethiopia. American Journal of Epidemiology, 115(2), 266-273.

G/hiwot, T. T., Sime, A. G., Deresa, B., Tafese, W., Hajito, K. W.,and Gemeda, D. H., 2016. Community Health Seeking Behavior for Suspected Human and Animal Rabies Cases, Gomma District, Southwest Ethiopia. Plos One, 11(3), e0149363. doi:10.1371/journal.pone.0149363

Hampson, K., Coudeville, L., Lembo, T., Sambo, M., Kieffer, A., Attlan, M., . . . Global Alliance for Rabies Control Partners for Rabies, P., 2015. Estimating the global burden of endemic canine rabies. PLoS Negl Trop Dis, 9(4), e0003709. doi:10.1371/journal.pntd.0003709

Jackson, A. C.,and Wunner, W., 2013. Rabies: scientific basis of the disease and its management. New York. 
Jemberu, W. T., Molla, W., Almaw, G.,and Alemu, S., 2013. Incidence of rabies in humans and domestic animals and people's awareness in North Gondar Zone, Ethiopia. PLoS Negl Trop Dis, 7(5), e2216. doi:10.1371/journal.pntd.0002216

Leta, S., and Mesele, F., 2014. Spatial analysis of cattle and shoat population in Ethiopia: growth trend, distribution and market access. SpringerPlus, 3(1), 310. doi:10.1186/2193-1801-3-310

Mengistu, F., Hussen, K., Ali, A., Getahun, G.,and Sifer, D., 2011. Dog bite as a public health concern in Addis Ababa. Ethiopian Journal of Health Development, 25(1), 58-60.

Nel, L. H., 2013. Discrepancies in data reporting for rabies, Africa. Emerging Infectious Diseases, 19(4), 529-533. doi:10.3201/eid1904.120185

OIE, 2011. World Organization for Animal Health. Rabies Control-Towards Sustainable Prevention at the Source." Compendium of the OIE Global Conference on Rabies Control. September 7-9, 2011. Incheon-Seoul (Republic of Korea).

Okell, C., Pinchbeck, G., Stringer, A., Tefera, G.,and Christley, R., 2013. A community-based participatory study investigating the epidemiology and effects of rabies to livestock owners in rural Ethiopia. Prev Vet Med, 108(1), 1-9.

Pankhurst, R. (1970). The history and traditional treatment of rabies in Ethiopia. Medical history, 14(04), 378-389.

Pieracci, E. G., Hall, A. J., Gharpure, R., Haile, A., Walelign, E., Deressa, A., . . Ermias, B., 2016. Prioritizing zoonotic diseases in Ethiopia using a one health approach. One Health, 2, 131-135. doi:http://dx.doi.org/10.1016/j.onehlt.2016.09.001.

Solomon, A., 2003. Livestock marketing in Ethiopia: a review of structure, performance, and development initiatives: ILRI (ILCA and ILRAD). Report series volume 52.

Teklu, G. G., Hailu, T. G.,and Eshetu, G. R., 2017. High Incidence of Human Rabies Exposure in Northwestern Tigray, Ethiopia: A Four-Year Retrospective Study. Plos Neglected Tropical Diseases, 11(1), e0005271. doi:10.1371/journal.pntd.0005271

Tefera, G., Yimer, E., Geyid, A., 2002. Endemic existence of rabies in Ethiopia. Eth. Med. J. l, 40(2), 163-170.

Tschopp, R., Bekele, S.,and Aseffa, A., 2016. Dog Demography, Animal Bite Management and Rabies Knowledge-Attitude and Practices in the Awash Basin, Eastern Ethiopia. Plos Neglected Tropical Diseases, 10(2), e0004471.

Vigilato, M. A. N., Ottorino Cosivi, Terezinha Knöbl, Alfonso Clavijo,and Silva, H. M. T., 2012. Rabies update for Latin America and the Caribbean. Morb Mortal Wkly Rep, 61, 213-214.

WHO, 2017. Human rabies: 2016 updates and call for data. Weekly Epidemiol Rec, 92(7), 7786.

Yimer, E., Mesfin, A., Beyene, M., Bekele, A., Taye, G., Zewdie, B.,and Alemayehu, T., 2012. Study on knowledge, attitude and dog ownership patterns related to rabies prevention and control in Addis Ababa, Ethiopia. Ethiopian Veterinary Journal, 16(2), 27-39. 
Yimer, E., Newayeselassie, B., Teferra, G., Mekonnen, Y., Bogale, Y., Zewde, B., . . B Bekele, A., 2002. Situation of rabies in Ethiopia: a retrospective study 1990-2000. Ethiopian Journal of Health Development, 16(1), 105-112.

Zinsstag, J., Schelling, E., Bonfoh, B., Fooks, A. R., Kasymbekov, J., Waltner-Toews, D.,and Tanner, M., 2009. Towards a 'One Health’research and application tool box. Veterinaria Italiana, 45(1), 121-133. 
Chapter 2

Systematic review on dog rabies vaccination coverage in Africa: A question of dog accessibility or cost recovery?

Tariku Jibat Beyene, Henk Hogeveen, Monique C.M. Mourits

Published in PLoS Negl Trop Dis 9(2): e0003447.

https://doi.org/10.1371/journal.pntd.0003447 


\begin{abstract}
Rabies still poses a significant human health problem throughout most of Africa, where the majority of the human cases results from dog bites. Mass dog vaccination is considered to be the most effective method to prevent rabies in humans. Our objective was to systematically review research articles on dog rabies parenteral vaccination coverage in Africa in relation to dog accessibility and vaccination cost recovery arrangement (i.e. free of charge or owner charged). A systematic literature search was made in the databases of CAB abstracts (EBSCOhost and OvidSP), Scopus, Web of Science, PubMed, Medline (EBSCOhost and OvidSP) and AJOL (African Journal Online) for peer reviewed articles on 1) rabies control, 2) dog rabies vaccination coverage and 3) dog demography in Africa. Identified articles were subsequently screened and selected using predefined selection criteria like year of publication (viz. $\geq 1990$ ), type of study (cross-sectional), objective(s) of the study (i.e. vaccination coverage rates, dog demographics and financial arrangements of vaccination costs), language of publication (English) and geographical focus (Africa). The selection process resulted in sixteen peer-reviewed articles which were used to review dog demography and dog ownership status, and dog rabies vaccination coverage throughout Africa. The main review findings indicate that 1) the majority (up to 98.1\%) of dogs in African countries are owned (and as such accessible), 2) puppies younger than 3 months of age constitute a considerable proportion (up to $30 \%$ ) of the dog population and 3) male dogs are dominating in numbers (up to 3.6 times the female dog population). Dog rabies parenteral vaccination coverage was compared between "free of charge” and "owner charged” vaccination schemes by the technique of Meta-analysis. Results indicate that the rabies vaccination coverage following a free of charge vaccination scheme (68\%) is closer to the World Health Organization recommended coverage rate (70\%) than the achieved coverage rate in owner-charged dog rabies vaccination schemes (18\%). Most dogs in Africa are owned and accessible for parenteral vaccination against rabies if the campaign is performed "free of charge".
\end{abstract}




\subsection{Introduction}

Rabies is one of the infectious diseases with the highest human case fatality rate (almost 100\%) (WHO, 2005). Globally, rabies is responsible for more than 60,000 human deaths, while approximately 15 million people receive rabies post-exposure prophylaxis (PEP) annually. More than 95\% of the global deaths occur in Asia and Africa, where canine rabies is enzootic (WHO, 2013). Africa contributes to $43 \%$ of the human deaths due to rabies (Knobel et al., 2005). In addition to human life losses, rabies is also a cause of substantial livestock losses (Okell et al., 2013) and a threat to rare carnivores like the Ethiopian wolf (Canis simensis) (Randall et al., 2004) and the African wild dog (Lycaon pictus) (Kat et al., 1996). Despite these consequences, rabies has been seriously neglected in Africa (Zinsstag, 2013).

The main cause of transmission of rabies to human in Africa is by a bite of a rabid dog (Aworh, 2011). Once bitten by a rabid dog, development of the disease in human can be prevented by an appropriate post-exposure prophylaxis (PEP). However, PEP is relatively expensive and not always available. Moreover, PEP lacks long-term benefits as it will not stop the virus transmission from rabid dogs to other humans or dogs (Bogel and Meslin, 1990). Dog rabies parenteral vaccination is therefore more cost-effective measure in preventing human rabies (Zinsstag et al., 2009).

To eliminate rabies from the dog population in an endemic area at least $70 \%$ of the dog population needs to be vaccinated during an annual rabies mass vaccination campaign (Coleman and Dye, 1996). In many African countries, the proportion of dogs vaccinated against rabies is far below 70\% (Cleaveland et al., 2003). Accessibility of free roaming dogs for vaccination is often mentioned as an operational constraint (Lembo et al., 2010) with the assumption that parenteral dog vaccination requires catching and restraining dogs physically. Catching free roaming dogs is easier if the dogs are owned. Therefore, dog ownership is an important factor in determining the percentage of dogs vaccinated during a campaign. Dog ownership status and management factors in developing countries in relation to dog rabies vaccination have extensively been addressed in literature (see for example (Davlin and VonVille, 2012)). But, as developing countries at different continents have a wide variation in social and cultural context, studies on African specific socio-economic situations related to dogs and rabies are a necessity for a valid interpretation and practical application of effective vaccination campaigns in Africa. Besides, there is no valid evidence to what extent charging owners for the costs of dog vaccination against rabies contribute to a low vaccination coverage.

Therefore, the objective of our study is to systematically review articles on parenteral vaccination coverage on dog rabies achieved in Africa, in relation to dog demographics and financial arrangements on vaccination costs. 


\subsection{Materials and Methods}

\subsubsection{Article search strategy}

To obtain insight in the trend of peer reviewed articles focussing on "dog rabies control in Africa” during the last 20 years (1994 - 2013), a systematic search was made in the databases of CAB abstracts (EBSCOhost and OvidSP), Scopus, Web of Science, PubMed, Medline (EBSCOhost and OvidSP) and AJOL (African Journal Online).

Subsequently, a search was made in the above mentioned databases for peer reviewed articles in the themes: 1) "rabies control”, 2) "dog vaccination coverage” and 3) "dog demography”. All theme searches were limited to papers regarding the continent of Africa. The search for each theme was conducted in the search items "title/abstract/key words" using the following search protocol: 1) ((dog? OR canine OR livestock OR human? OR wild? life) AND rabies AND control AND Africa?), 2) ((dog? OR canine) AND rabies AND vaccine* AND coverage AND Africa?) and 3) ((dog OR canine) AND (demography OR population) AND Africa?). The search protocol was designed, based on standard procedures of a systematic literature search (Gough, 2012). However, as the AJOL database webpage has no feature to select the search protocol in title/abstract/keywords, the search in AJOL was done within the entire article. The systematic literature search included articles published between 1990 and January 2014.

\subsubsection{Framework for screening and selection criteria}

Publications were screened systematically according to the schematic framework as shown in Figure 1 using EndNote X5 (Endnote @ 2013) reference manager. First, an evaluation of titles and abstracts was performed followed by a removal of duplicates (i.e., publications indexed in more than one databases and published in more than one format, including conference proceedings and book chapters). Several inclusion and exclusion criteria were considered including year of publication (viz. $\geq 1990$ ), type of study (cross sectional), objective(s) of the study (parenteral vaccination coverage rates on dog rabies in Africa, dog demographics and financial arrangements with respect to vaccination costs), language of publication (English) and geographical focus (Africa). As a result, a publication could be excluded for more than one reason, making it impractical to reflect the number of publications excluded per criteria. Articles, of which the full text was not electronically available, were requested from the Royal College of Veterinary Surgeons Trust Library (in United Kingdom). For each selected article, a record was made in Microsoft Excel describing the studied dog population by the main purpose for keeping dogs, dog demography (mean age, age distribution, sex ratio age and sex distribution), ownership status (percentage of owned, free roaming and ownerless dogs), and parenteral vaccination coverage based on either "free of charge" or "owner charged" financial arrangements. The selected articles encompassed research from Southern (South Africa, Madagascar, Zimbabwe, Zambia), Central (Chad), Northern (Tunisia), Eastern (Tanzania, Kenya, Ethiopia) and Western (Nigeria) Africa. The methodological quality of candidate peer 
review articles was critically assessed by the Assessment of Multiple Systematic Reviews (AMSTAR) measurement tool (Shea et al., 2007, Moher et al., 2009) and (Supporting information: Checklist 2.1).

\subsubsection{Statistical analyses}

Results on dog demography (i.e. sex ratio and mean age of dogs) and ownership status were extracted from the selected articles and presented in tabular form without further analysis. In a meta-analysis, we evaluated the difference in the percentage of dogs vaccinated against rabies (i.e. vaccination coverage) by the applied financial arrangement on vaccination costs; i.e. whether the vaccination was provided for free to the dog owners or not (i.e. free versus charged). From the selected articles the presented parenteral vaccination coverage was entered as an event rate in the meta-analysis software (Comprehensive meta-analysis V2, 2013). A Forest plot was created to serve as a visual representation of the data in a combined point estimate for the free and charged vaccination study groups, bounded by its confidence interval. Statistical differences, called heterogeneity tests, between the two groups of studies were tested as indicated by $\mathrm{I}^{2}$ and tau square. $\mathrm{I}^{2}$ represents the percentage of the total variation across studies due to heterogeneity across studies within a group and across a group. It takes values from $0 \%$ to $100 \%$, with the value of $0 \%$ indicating no observed heterogeneity. Tau square is an estimate of the between study and between group variance. If greater than 1 , it suggests the presence of substantial statistical heterogeneity in each group, which is a statistical variation due to heterogeneity rather than chance between the free-of-charge and charged study groups (Egger and Smith, 1997, Higgins JP, 2003). 


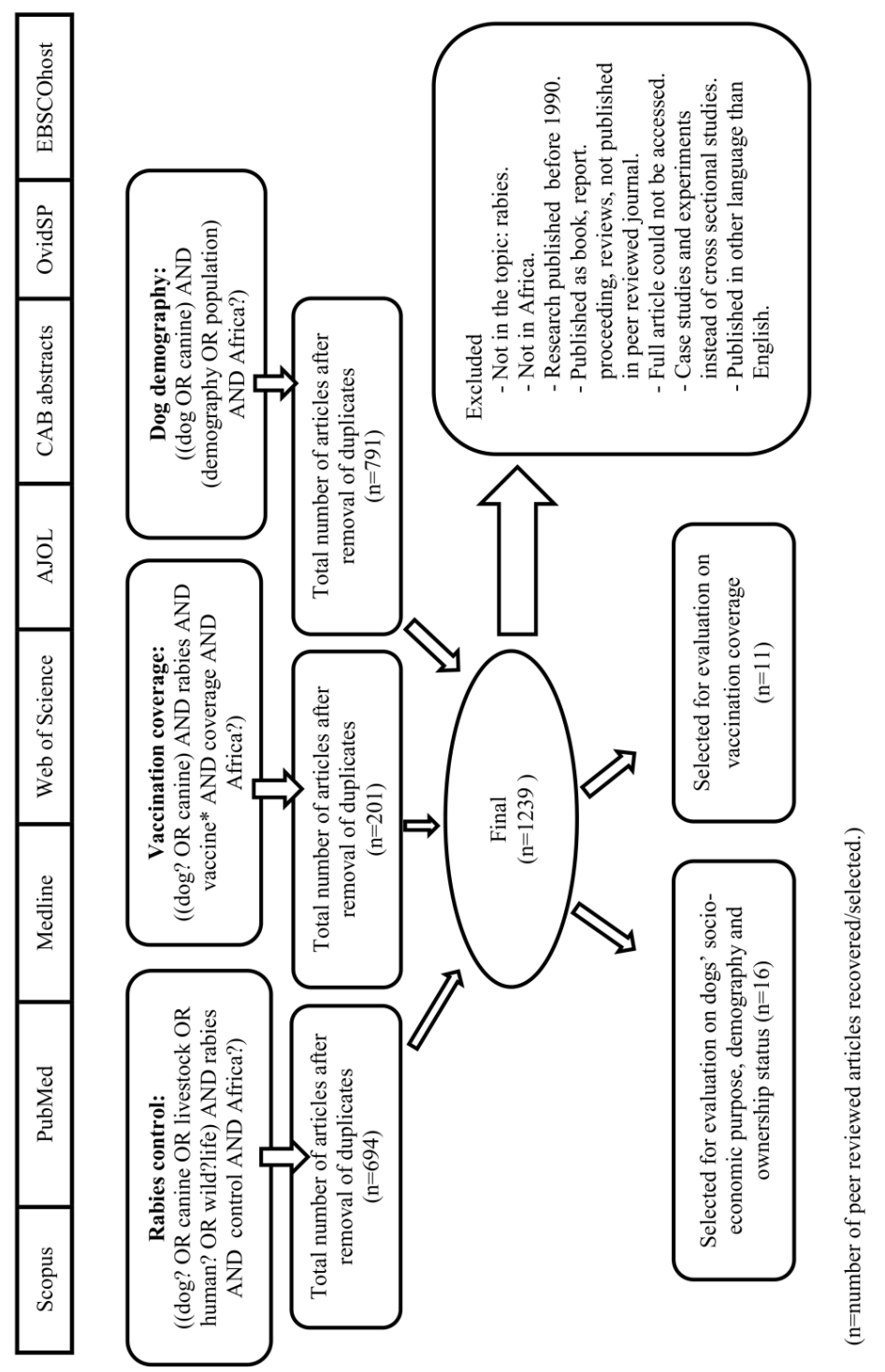

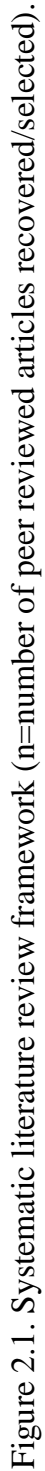




\subsection{Results}

\subsubsection{Literature search study}

Based on the systematic literature search using the phrase "Rabies control in Africa", the highest number of research publications was found indexed by the Web of Science/Knowledge database. In Figure 2 the trend in the number of scientific publications on the topics "Rabies in Africa”, "Dog/Canine rabies in Africa” and "Control of "Dog/Canine rabies in Africa" during the last 20 years indicates an increase in scientific interest on rabies in Africa (Figure 2). While a worldwide search on "Rabies" during the same period resulted in 9,836 selected entries, only 328 of them were specifically referring to the African situation. Of these 328 papers, approximately half focussed specifically on dog rabies ("Dog/Canine rabies in Africa”, n=172) and one fifth on dog rabies control ("Control of "Dog/Canine rabies in Africa”, n=76).

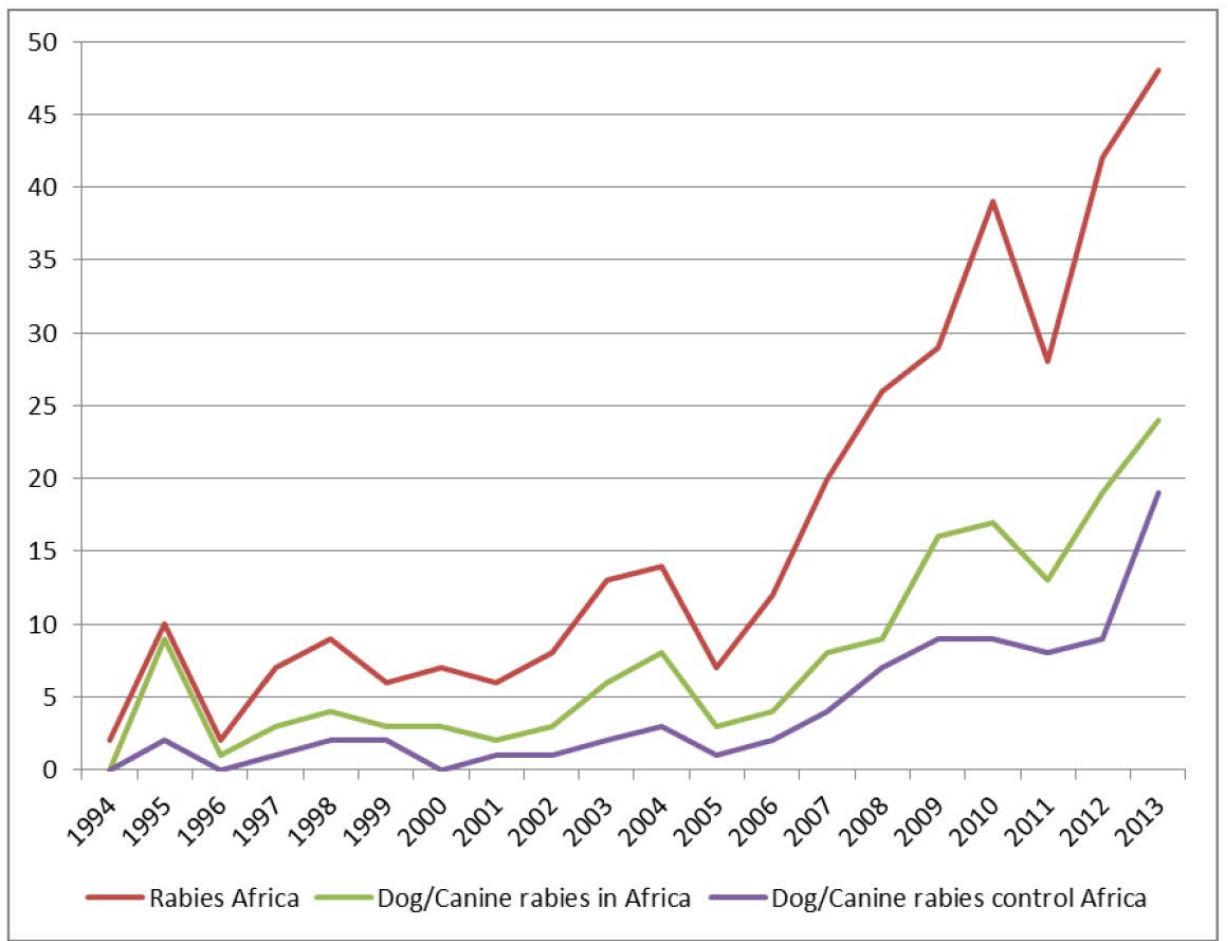

Figure 2.2 Trend in number of scientific publications on "Rabies in Africa”, "Dog/Canine rabies in Africa" and "Control of "Dog/Canine rabies in Africa" as indexed by Web of Science/Knowledge during the last 20 years.

The systematic search from the databases by the defined framework resulted in 1,239 articles (Figure 2.1). After removal of all duplicates and exclusion of publications not fulfilling the selection criteria, 16 peer reviewed articles remained to be included in the study on dog 
demography (sex ratio and mean age of dogs) and ownership status (owned confined, owned but free roaming and proven to be ownerless). Seven of these papers have been published during the last five years. For the comparison of dog parenteral vaccination coverage against rabies related to the dog owners' costs of vaccination, 11 peer reviewed articles with 15 entries (including four studies comparing free and owner charged vaccination arrangements) remained. The majority of these papers (7 out of 11) has been recently published (e.g. between 2009 and 2013).

\subsubsection{Socio-economic purpose, demography and ownership status of dogs}

The majority (up to 98\%) of dogs in African countries is kept for socio-economic purposes including guarding livestock from predators, homestead from intruders, crops from wildlife and hunting. Dogs are also used as pets, income generation means and as a protein source (Table 2.1) (Ratsitorahina et al., 2009; Knobel et al., 2008; Yimer et al., 2012; Aiyedun and Olugasa, 2012). Furthermore, puppies younger than 3 months of age constitute up to $30 \%$ of the dog population (Gsell et al., 2012). Male dogs dominate the female dogs up to 3.6 times in number within the population (Yimer et al., 2012). The mean age of the dogs varies between 1.8 and 3.4 years. Studies accounting for ownership of dogs (Table 2.1) showed that the percentage of ownerless dogs ranges between $0.7 \%$ and $20 \%$ of a dog population within the 11 represented African countries. Except for a study in Tanzania (Gsell et al., 2012), all studies reported that more than two third of the free roaming dogs has a responsible owner (Ratsitorahina et al., 2009; Yimer et al., 2012, Aiyedun and Olugasa, 2012; Van Sittert et al., 2010; Kitala et al., 2001; Kaare et al., 2009). Owned dogs with confined housing constitute $18.5 \%$ to $60.9 \%$ of the dog population. 


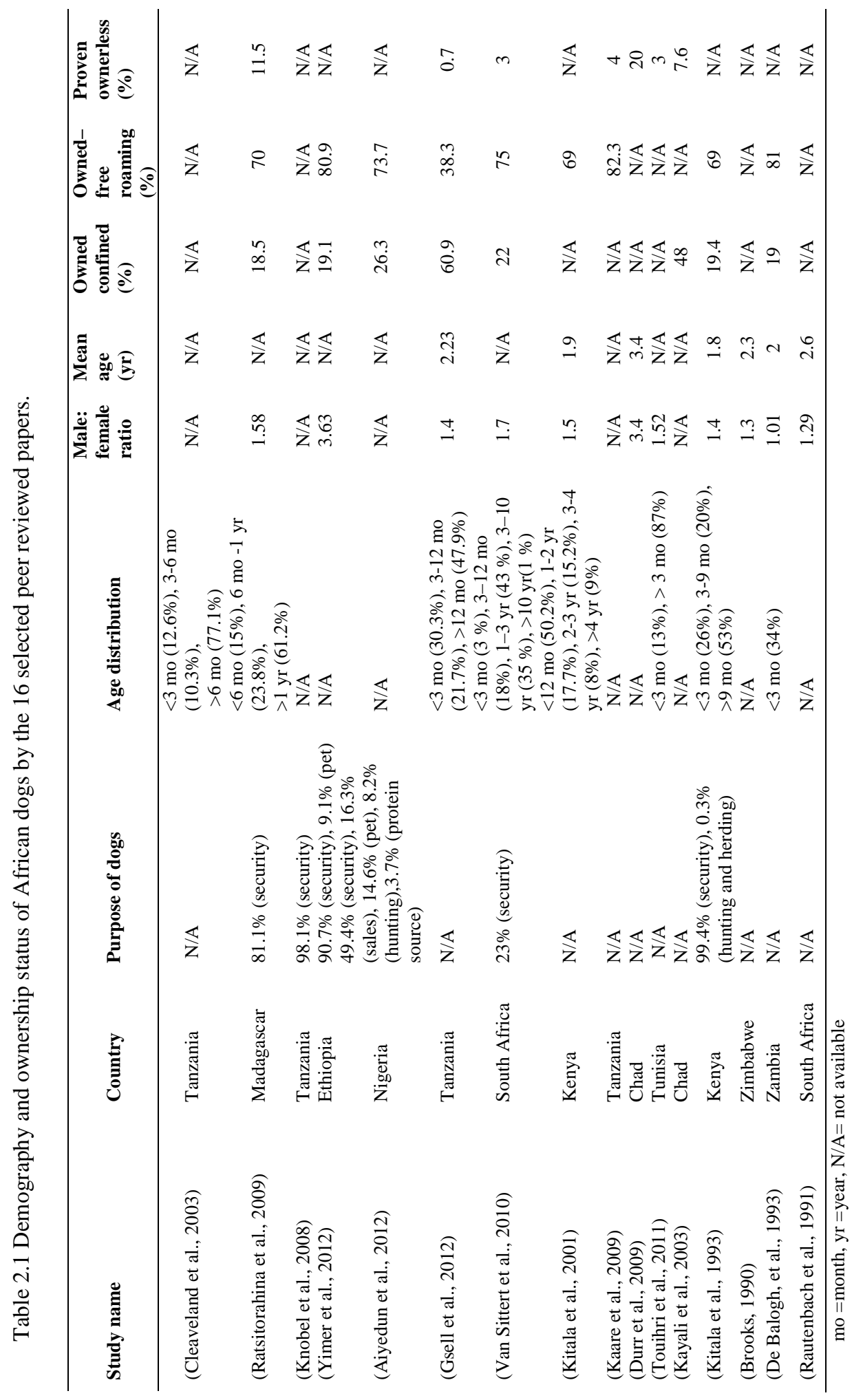




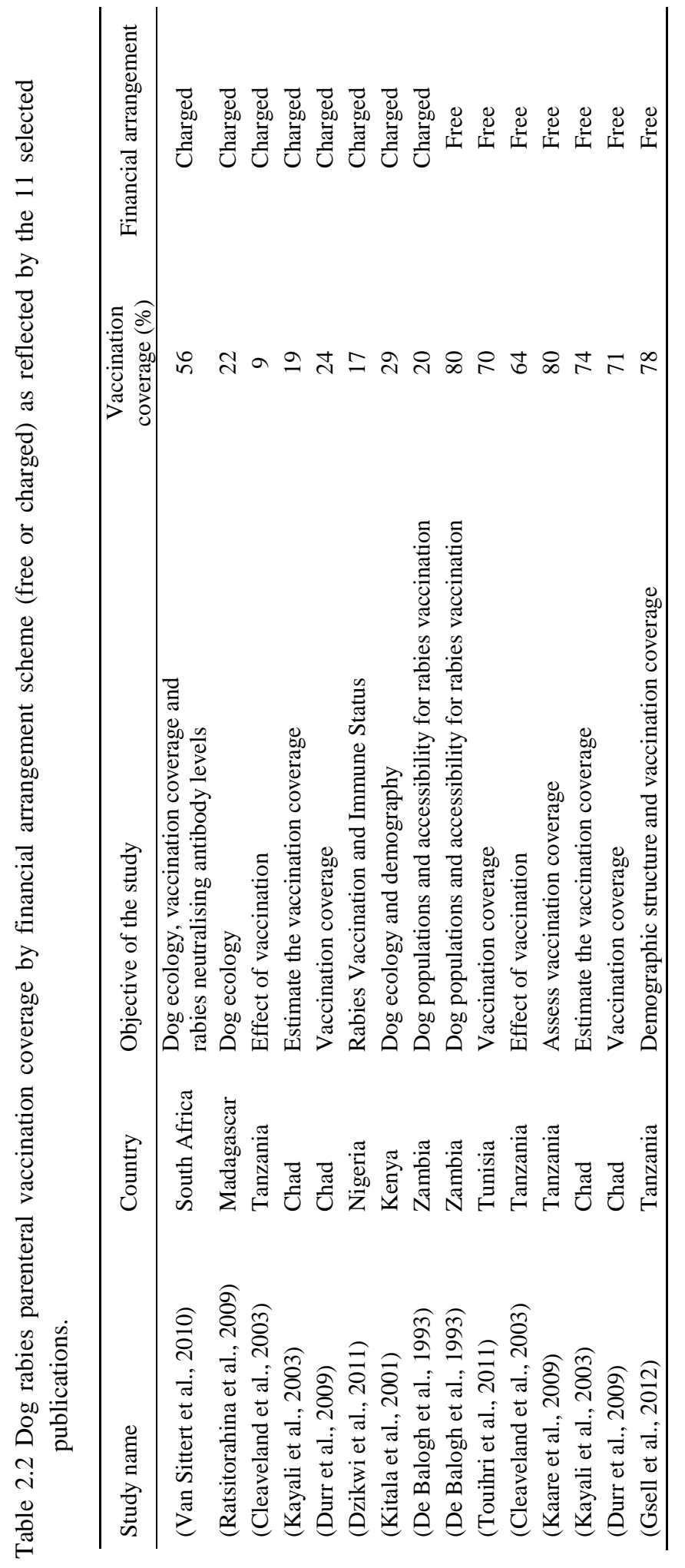




\subsubsection{Coverage rate by free versus charged parenteral mass dog vaccination schemes}

The published studies selected for vaccination coverage comparison by vaccination costs arrangement schemes consisted of eleven studies in eight different countries representing all regions of Africa (Table 2.2). Four studies compared vaccination coverage under "free of charge" and "charged" arrangements schemes, four studies evaluated vaccination coverage resulting from "charged” vaccination arrangement schemes only and three studies estimated parenteral vaccination coverage by a "free-of-charge" scheme. The Forest plot (Figure 2.3) shows a coverage of less than $50 \%$ in the charged groups except for one study, while all studies under free of charge arrangements resulted in a coverage above $50 \%$. The vaccination coverage in studies based on free of charge vaccination $(68 \%)$ is significant higher $(\mathrm{p}<0.001)$ than the studies based on a charged vaccination campaign (18.1\%).

Table 2.3 Statistical heterogeneity tests comparing vaccination coverage rates resulting from charged versus free-of-charge vaccination schemes

\begin{tabular}{|c|c|c|c|c|c|c|}
\hline \multirow[t]{2}{*}{ Groups } & \multirow[t]{2}{*}{ No. studies } & \multicolumn{3}{|c|}{ Vaccination coverage and 95\% CI } & \multicolumn{2}{|c|}{ Heterogeneity } \\
\hline & & Point estimate & $\begin{array}{l}\text { Lower } \\
\text { limit }\end{array}$ & $\begin{array}{l}\text { Upper } \\
\text { limit }\end{array}$ & $\mathrm{I}^{2}$ & $\mathrm{Tau}^{2}$ \\
\hline Charged & 8 & 0.18 & 0.17 & 0.19 & 98.45 & 0.48 \\
\hline Free & 7 & 0.68 & 0.67 & 0.69 & 98.22 & 0.16 \\
\hline Overall & 15 & 0.55 & 0.55 & 0.56 & 99.75 & 1.49 \\
\hline
\end{tabular}

\begin{tabular}{ll} 
Group by & Study name \\
\cline { 1 - 1 } Comparison & \\
Charged & Van Sittert et al., 2010 \\
Charged & Ratsitorahina et al., 2009 \\
Charged & Cleaveland et al., 2003 \\
Charged & Kayali et al., 2003 \\
Charged & Durr et al., 2009 \\
Charged & Dzikwi et al., 2011 \\
Charged & Kitala et al., 2001 \\
Charged & De Belogn et al., 1993 \\
Free & \\
Free & Cleaveland et al., 2003 \\
Free & Kayali et al., 2003 \\
Free & Durr et al., 2009 \\
Free & Touihri et al., 2011 \\
Free & Kaare et al., 2009 \\
Free & Gsell et al., 2012 \\
Free & De Balogh et al., 1993 \\
Overall & \\
& \\
\hline
\end{tabular}

\begin{tabular}{|c|c|c|c|}
\hline & tatistics & 5 for each study & \\
\hline $\begin{array}{c}\text { Event } \\
\text { rate }\end{array}$ & $\begin{array}{l}\text { Lower } \\
\text { limit }\end{array}$ & $\begin{array}{l}\text { Upper } \\
\text { limit }\end{array}$ & p-Value \\
\hline 0.560 & 0.491 & 0.627 & \\
\hline 0.216 & 0.200 & 0.233 & \\
\hline 0.091 & 0.083 & 0.100 & \\
\hline 0.190 & 0.157 & 0.228 & \\
\hline 0.240 & 0.215 & 0.267 & \\
\hline 0.169 & 0.122 & 0.229 & \\
\hline 0.290 & 0.242 & 0.343 & \\
\hline 0.202 & 0.159 & 0.254 & \\
\hline 0.181 & 0.173 & 0.189 & 0.000 \\
\hline 0.639 & 0.630 & 0.648 & \\
\hline 0.736 & 0.694 & 0.774 & \\
\hline 0.710 & 0.681 & 0.737 & \\
\hline 0.700 & 0.676 & 0.723 & \\
\hline 0.803 & 0.783 & 0.822 & \\
\hline 0.778 & 0.761 & 0.794 & \\
\hline 0.800 & 0.748 & 0.843 & \\
\hline 0.680 & 0.673 & 0.687 & 0.000 \\
\hline 0.556 & 0.550 & 0.563 & 0.000 \\
\hline
\end{tabular}

Event rate and $95 \% \mathrm{Cl}$

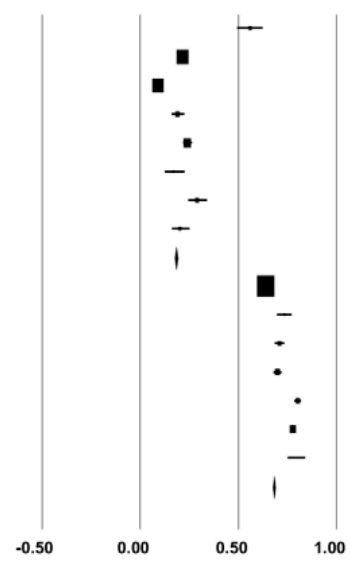

Figure 2.3 Forest plot comparing dog rabies vaccination coverage by financial arrangement 
Table 2.3 provides the heterogeneity test results from the two groups of vaccination cost arrangement schemes. $\mathrm{I}^{2}$ describes the percentage of variation across groups due to heterogeneity rather than chance. This study shows $99.9 \%$ heterogeneity between free and owner charged groups, indicating significant difference between the vaccination coverage in the two groups. Tau square is an estimate of the between-study variance in the meta-analysis. As Tau square between studied groups is larger than one (i.e. 1.45), it shows a substantial heterogeneity between the studied groups, while Tau squares within the free and charged groups were smaller than one ( 0.16 and 0.54 , respectively).

\subsection{Discussion}

According to the World Health Organization (WHO), the adequate vaccination coverage of a dog population in a community vaccinated annually against rabies should be at least $70 \%$ in order to block the occurrence of an outbreak (WHO, 2005). In this study dog accessibility for parenteral vaccination reflected by the ownership status and vaccination costs arrangement schemes were assessed to explore their influence on the realised vaccination coverage in Africa.

When resources have to be allocated to the control of a disease, this should be done on scientific evidence. For instance, the organized efforts of the Pan American Health Organization (PAHO) in Latin America (Schneider et al., 2007) and the Bohol Rabies Prevention and Elimination Project of the Philippines (Lapiz et al., 2012) have witnessed the possibility of reducing the incidence and burden of rabies with concerted efforts of experts. In Africa also, as growing scientific interest was shown through publications produced in the last few decades, it is possible to control rabies with organization of resources from different stakeholders together with a high local community involvement.

Accessibility of dogs is perceived to be the major operational constraint to achieve adequate coverage for dog vaccination against rabies through mass dog vaccination schemes(Lembo $\mathrm{T}$ et al., 2010). Our study shows that the majority of dogs in Africa is free roaming but owned. Dogs having responsible owners are accessible for parenteral mass vaccination indicating the possibility of achieving the minimum proportion of dogs that ought to be vaccinated to reduce the incidence of rabies. However, it doesn't mean that all owned dogs are presented for vaccination(Wera E, 2013). Oral rabies vaccination could be an option for those dogs that are difficult to capture, whether these dogs are owned or ownerless (WHO, 1998; Ben Youssef et al., 1998). As long as the proportion of ownerless dogs is less than $20 \%$ it is still possible to obtain sufficient immunity coverage by focussing on the mass vaccination of owned dogs. The relative impact of ownerless dogs could be studied by looking at the proportion of ownerless dogs compared to owned dogs in reported cases of human bites. For instance, in Nigeria only 9.7\% of the dog bites could not be traced back to a dog with a responsible owner (Bata et al., 2011). A study in South Africa showed that only a small proportion of dog bite reports resulted 
from unknown dogs (Hergert and Nel, 2013). In Chad, only 3\% of the biting dogs were ownerless or from an unknown owner (Kayali et al., 2003).

Studies referred in this review showed that the mean age of the African dog is between 1.9 and 3.4 years indicating an average turnover rate between $53 \%$ and $29 \%$. These numbers are higher when compared to the turnover rates in industrialized countries as for instance, in North America and Europe where the dogs have an average life expectancy of respectively 4.5 years (Wandeler et al., 1988) and 5.7 years (Egenvall et al., 1999) resulting in an average turnover rate of $20 \%$. Insight in the dog population demography, population size and turnover rates supports the selection of a vaccine in a vaccination scheme in terms of protection time period and frequency of required boosting to keep the required level of immunity.

Male dogs represent a considerable higher proportion of the African owned dog population than female dogs. Male dogs are more aggressive than female dogs and are, therefore, preferred for guarding and hunting. This might have implications in the transmission of rabies to humans and other dogs. For instance, a study in Chad showed that $80 \%$ of the human dog bites originated from male dogs (Frey et al., 2013). Male dogs are also more likely to be diagnosed positive for rabies than females (Durr et al., 2008).The risk of acquiring an infection may possibly be influenced by males' fighting over females during the breeding season (Ali et al., 2010).

According to the performed meta-analysis, the vaccination costs recovery arrangement is one of the factors determining the proportion of dogs vaccinated against rabies in a community. In most African countries, where dog vaccination is not free of charge, the coverage is as low as $9 \%$ (Tanzania) (Cleaveland et al., 2003). The willingness of dog owners to pay for dog rabies vaccination is generally low as they perceive no direct economic and/or health benefit for themselves. This is supported by the knowledge that the majority of humans bitten were not bitten by their own dogs (Lapiz et al., 2012; Hergert and Nel, 2013).

The majority of the reviewed studies on charged vaccination schemes did not stated explicitly which costs were charged from the dog owners. In the study of Dürr et al. (Durr et al., 2009) in Chad, it was indicated that only $24 \%$ of the dogs was vaccinated during a parenteral mass dog vaccination campaign in which owners had to pay $21 \%$ of the vaccination costs themselves. Dog owners in Chad (Durr et al., 2008) were willing to pay $\approx 400-700$ CFA francs per animal, while the average vaccination costs corresponded to 4000 CFA francs per animal (e.g. 10$17.5 \%$ of total costs). These findings also indicate the need for substantial subsidise to vaccinate the required $>70 \%$ of dogs to interrupt rabies transmission.

Within the African context, the percentage of dogs vaccinated under free of charge vaccination schemes is much higher compared to the owner charged vaccination coverage. However, in 
developing countries in Asia, like Indonesia, a different situation was observed. Despite the application of a free of charge vaccination campaign (Wera et al., 2013) vaccination coverage remained as low as 33\%. This difference might be explained by the diverse levels of awareness, beliefs and socio-economic factors among the different continents. Similarly, studies in Asia showed that educational level, dog ownership and veterinary service access are important factors affecting the vaccination coverage (Lapiz et al., 2012; Tenzin et al., 2012). The estimation of percentage of dogs vaccinated in owner charged schemes might have been slightly overestimated compared to the vaccination coverage in costs free schemes, because only owned dogs are considered in the case of owner charged vaccination schemes.

Despite the fact that puppies younger than 3 months of age are generally excluded from vaccination campaigns (Durr et al., 2009), they contribute to a significant proportion of the dog population. This will influence the vaccination coverage and also the risk of human rabies especially the risk for children who have more frequent contacts with puppies than adults. The general exclusion of this age group is often a result of acting upon vaccine manufactures' guidelines and recommendations. Many rabies vaccines are licensed and approved for primary vaccination of dogs older than 3 months of age. However, it has been shown that most young puppies born from non-vaccinated mothers develop protective antibody titres after a vaccination as early as 4 weeks of age (Dzikwi et al., 2011; Aghomo et al., 1990).

A high vaccination coverage rate might not necessarily guarantee an effective rabies control. Studies have shown that African dogs experience reduced sero-conversions after rabies vaccination. The study of, for instance, indicated that only $71.9 \%$ of the vaccinated dogs in Nigeria developed neutralizing antibodies to rabies virus. The antibody titre depends on the time from vaccination, the technical efficacy of the vaccine used, the nutritional status, sex and age of the dogs (Kennedy et al., 2007; Moreno et al., 2012) also on the quality of the vaccine conservation. Furthermore, vaccination must be repeated more than once to effectively control rabies in a dog population.

A limitation of this study is the exclusion of unpublished reports and non-English articles in the review. Assessment of publications such as country reports of the Southern and Eastern Africa rabies group (SEARG) and the African Rabies Expert Bureau (AfroREB) could have provided additional information. However, as comparable reports are lacking or not accessible due to language barriers it could have created bias if only those country reports were included that were publicly accessible. Therefore, we focused our literature search on objective scientific articles that are internationally accessible.

The language selection criteria also excluded peer reviewed research papers published in French. This is could be a serious limitation of our study due to the fact that French is a very 
common language in African countries. A comparable systematic search in the French literature resulted in a selection of only 28 French papers (compared to the 1,239 hits resulted from the English search). Based on an evaluation of the English abstracts, only two publications (Zinsstag et al., 2011; Mindekem et al., 2005) fulfilled the remainder selection criteria as applied in this study. Main findings as presented in the abstracts of these papers were completely in line with the presented results.

\subsection{Conclusion}

This review provided a comprehensive account on the dog rabies parenteral vaccination coverage, dog demography and ownership within the African situation. The main findings of this systematic review indicate that 1 ) there has been a growing scientific interest in dog rabies control in Africa during the last two decades, which reflects a positive development given the argument that scientific evidence dictates stakeholders in allocation of resources for control and prevention of infectious diseases, 2) only a small proportion of the African dog population is ownerless and, 3) puppies younger than 3 months of age constitute a considerable proportion of the African dog population, 4) male dogs are dominant in the African dog population and 5) the proportion of dogs vaccinated against dog rabies when vaccination is free of charge is closer to WHO recommendations compared to owner charged vaccination schemes. Therefore, as most dogs in Africa are owned and therefore accessible for parenteral vaccination, a high vaccination coverage can be obtained once the necessary financial arrangements are arranged through organized community participation and/or public funding arrangements. 


\subsection{References}

Aghomo HO, O. O., Rupprecht CE, . 1990. The serological response of young dogs to the Flury LEP strain of rabies virus vaccine. Vet Res Commun, 14(5), 415-425.

Aiyedun, J. O., Olugasa, B. O. 2012. Identification and analysis of dog use, management practices and implications for rabies control in Ilorin, Nigeria. Sokoto Journal of Veterinary Sciences, 10(2). doi:10.4314/sokjvs.v10i2.1

Ali A, Y. E., Mengistu F, Hussen K, Tafese K. 2010. Overview of Rabies in and around Addis Ababa, in Animals Examined in EHNRI Zoonoses Laboratory Between, 2003 and 2009. Ethiop. Vet. J., 2010, 14(2), 91-101.

Aworh, M. K., Nwosuh C., Ajumobi, O.O., Okewole, P.A., Okolocha, E.C., Akanbi B.O., Nguku P. 2011. A Retrospective study of rabies cases reported at Vom Christian Hospital, Plateau State, Nigeria, 2006 - 2010. Nigerian Veterinary Journal, VOL:32 (4) 366-370. doi:10.1016/S0140-6736(14)60497-9

Bata SI, Dzikwi AA, Ayika, D. 2011. Retrospective study of dog bite cases reported to ECWA veterinary clinic, Bukuru, plateau state, Nigeria. . Science World Journal., 6(4 ), 17-19.

Ben Youssef S, M. H., Schumacher CL, Kharmachi H, Jemli J, et al., Mrabet, L., Gharbi, M., Hammami, S., El Hicheri, K., Aubert, M. F., Meslin, F. X. 1998. Field evaluation of a dog owner, participation-based, bait delivery system for the oral immunization of dogs against rabies in Tunisia. Am J Trop Med Hyg, 58(6), 835-845.

Bogel, K., Meslin, F.-X. 1990. Economics of human and canine rabies elimination:guidelines for programme orientation*. Bulletin of the World Health Organization, 66(3), 281-291.

Brooks, R. 1990. Survey of the dog population of Zimbabwe and its level of rabies vaccination. Vet Rec, 127(24), 592-596.

Cleaveland, S., Kaare, M., Tiringa, P., Mlengeya, T., Barrat, J. 2003. A dog rabies vaccination campaign in rural Africa: impact on the incidence of dog rabies and human dog-bite injuries. Vaccine, 21(17-18), 1965-1973. doi:10.1016/s0264-410x(02)00778-8

Coleman, P. G., \& Dye, C. 1996. Immunization coverage required to prevent outbreaks of dog rabies. Vaccine, 14(3), 185-186.

Davlin, S. L., VonVille, H. M. 2012. Canine rabies vaccination and domestic dog population characteristics in the developing world: a systematic review. Vaccine, 30(24), 34923502. doi:http://dx.doi.org/10.1016/j.vaccine.2012.03.069.

De Balogh, K. K., Wandeler, A. I., Meslin, F. X. 1993. A dog ecology study in an urban and a semi-rural area of Zambia. Onderstepoort J Vet Res, 60(4), 437-443.

Durr S, M. M., Mindekem R, Zinsstag J. (008. Owner valuation of rabies vaccination of dogs, Chad. Emerg Infect Dis, 14(10), 1650-1652. doi:10.3201/eid1410.071490

Durr S, M. R., Kaninga Y, Moto D, Doumagoum MD, et al., Meltzer, M. I., Vounatsou, P., Zinsstag, J. 2009. Effectiveness of dog rabies vaccination programmes: comparison of owner-charged and free vaccination campaigns. Epidemiology and Infection, 137(11), 1558-1567. doi:10.1017/s0950268809002386 
Durr S, N. S., Mindekem R, Diguimbye C, Niezgoda M, et al., Kuzmin, I., Rupprecht, C. E., Zinsstag, J. 2008. Rabies diagnosis for developing countries. PLoS Negl Trop Dis, 2(3), e206. doi:10.1371/journal.pntd.0000206

Dzikwi AA, U. J., Kwaga JKP, Ahmad AA. 2011. Rabies vaccination and immune status of owned dogs in Zaria, Nigeria. Nigerian Veterinary Journal, 32(3), 204-207.

Egenvall A, H. A., Bonnett BN, Olson P, 1999. Survey of the Swedish dog population: age, gender, breed, location and enrollment in animal insurance. Acta Vet Scand, 40(3), 231240.

Egger, M., Smith, G. D. 1997. Meta-Analysis. Potentials and promise. Bmj, 315(7119), 13711374.

Frey J, M. R., Kessely H, Doumagoum MD, Naissengar S, et al., Zinsstag, J., Schelling, E. 2013. Survey of animal bite injuries and their management for an estimate of human rabies deaths in N'Djamena, Chad. Trop Med Int Health, 18(12), 1555-1562. doi:10.1111/tmi.12202

Gough DOS, T. J. 2012. An Introduction to systematic reviews, . SAGE publications Inc., p. 107-135.

Gsell AS, K. D., Kazwala RR, Vounatsou P, Zinsstag J. 2012. Domestic dog demographic structure and dynamics relevant to rabies control planning in urban areas in Africa: the case of Iringa, Tanzania. BMC Veterinary Research, 8, 1-10. doi:10.1186/1746-61488-236

Hergert, M., Nel, L. H. 2013. Dog bite histories and response to incidents in canine rabiesenzootic KwaZulu-Natal, South Africa. PLoS Negl Trop Dis, 7(4), e2059. doi:10.1371/journal.pntd.0002059

Higgins JP, T. S., Deeks JJ, Altman DG. 2003. Measuring inconsistency in meta-analyses. BMJ, 327(7414), 557-560. doi:10.1136/bmj.327.7414.557

Kaare M, L. T., Hampson K, Ernest E, Estes A, et al., Mentzel, C., Cleaveland, S. 2009. Rabies control in rural Africa: Evaluating strategies for effective domestic dog vaccination. Vaccine, 27(1), 152-160. doi:10.1016/j.vaccine.2008.09.054

Kat, P. W., Alexander, K. A., Smith, J. S., Richardson, J. D., Munson, L. 1996. Rabies among African wild dogs (Lycaon pictus) in the Masai Mara, Kenya. J Vet Diagn Invest, 8(4), 420-426.

Kayali U, M. R., Yemadji N, Oussiguere A,Naissengar S, , Ndoutamia, A. G., Zinsstag, J. 2003. Incidence of canine rabies in N'Djamena, Chad. Prev Vet Med, 61(3), 227-233.

Kayali U, M. R., Yemadji N, Vounatsou P, Kaninga Y, et al., Ndoutamia, A. G., Zinsstag, J. 2003. Coverage of pilot parenteral vaccination campaign against canine rabies in N'Djamena, Chad. Bull World Health Organ, 81(10), 739-744.

Kennedy LJ, L. M., Barnes A, McElhinney L, Fooks AR, et al., Baxter, D. N., Ollier, W. E. 2007. Factors influencing the antibody response of dogs vaccinated against rabies. Vaccine, 25(51), 8500-8507. doi:10.1016/j.vaccine.2007.10.015 
Kitala P, M. J., Kyule M, Gathuma J, Perry B, et al., Wandeler, A. 2001. Dog ecology and demography information to support the planning of rabies control in Machakos District, Kenya. Acta Tropica, 78(3), 217-230. doi:10.1016/s0001-706x(01)00082-1

Kitala PM, M. J., Kyule MN, Cathuma JM, . 1993. Features of dog ecology relevant to rabies spread in Machakos District, Kenya. Onderstepoort J Vet Res, 60(4), 445-449.

Knobel, D. L., Cleaveland, S., Coleman, P. G., Fevre, E. M., Meltzer, M. I., Miranda, M. E. G., ... Meslin, F.-X. 2005. Re-evaluating the burden of rabies in Africa and Asia. Bulletin of the World Health Organization, 83(5), 360-368.

Knobel DL, L. M., Kazwala RR, Boden L, Cleaveland S. 2008. A cross-sectional study of factors associated with dog ownership in Tanzania. BMC Veterinary Research, 4, 5. doi:1746-6148-4-5 [pii]10.1186/1746-6148-4-5.

Lapiz SM, M. M., Garcia RG, Daguro LI, Paman MD, et al., Madrinan, F. P., Rances, P. A., Briggs, D. J. 2012. Implementation of an intersectoral program to eliminate human and canine rabies: the Bohol Rabies Prevention and Elimination Project. PLoS Negl Trop Dis, 6(12), e1891. doi:10.1371/journal.pntd.0001891

Lembo T, H. K., Kaare MT, Ernest E, Knobel D, et al., Kazwala, R. R., Haydon, D. T., Cleaveland, S. 2010. The feasibility of canine rabies elimination in Africa: dispelling doubts with data. PLoS Negl Trop Dis, 4(2), 0000626.

Mindekem R, K. U., Yemadji N, Ndoutamia AG, Zinsstag J. 2005. [Impact of canine demography on rabies transmission in N'djamena, Chad]. Médecine tropicale : revue du Corps de santé colonial, 65(1), 53-58.

Moher D, Liberati A, Tetzlaff J, Altman DG, \& ., T. P. G. 2009. Preferred Reporting Items for Systematic Reviews and Meta-Analyses: The PRISMA Statement. PLoS Med, 6((6): ), e1000097. doi:1000010.1001371/journal.pmed1000097.

Moreno J, B. N., Piaggio J, Puentes R. 2012. Canine immune response to rabies vaccination. . Veterinaria, 48(186), p. 19-22.

Okell, C. N., Pinchbeck, G. P., Stringer, A. P., Tefera, G., Christley, R. M. 2013. A communitybased participatory study investigating the epidemiology and effects of rabies to livestock owners in rural Ethiopia. Preventive Veterinary Medicine, 108(1), 1-9. doi:10.1016/j.prevetmed.2012.07.003

Randall, D. A., Williams, S. D., Kuzmin, I. V., Rupprecht, C. E., Tallents, L. A., Tefera, Z., . . . Laurenson, M. K. 2004. Rabies in endangered Ethiopian wolves. Emerg Infect Dis, 10(12), 2214-2217. doi:10.3201/eid1012.040080

Ratsitorahina M, R. J., Raharimanana S, Rakotonandrasana H, Andriamiarisoa M, et al., Rakalomanana, F. A., Richard, V. 2009. Dog ecology and demography in Antananarivo, 2007. BMC Veterinary Research, 5, 21. doi:1746-6148-5-21 [pii]10.1186/1746-61485-21 [doi] 
Rautenbach, G. H., Boomker, J., de Villiers, I. L. 1991. A descriptive study of the canine population in a rural town in southern Africa. Journal of the South African Veterinary Association, 62(4), 158-162.

Schneider MC, B. A., Ade MP, Hendrickx S, Leanes LF, et al., Rodrigues, M. J., Medina, G., Correa, E. 2007. Current status of human rabies transmitted by dogs in Latin America. Cad Saude Publica, 23(9), 2049-2063.

Shea BJ, G. J., Wells GA, Boers M, Andersson N, et al., Hamel, C., Porter, A. C., Tugwell, P., Moher, D., Bouter, L. M. 2007. Development of AMSTAR: a measurement tool to assess the methodological quality of systematic reviews. BMC Med Res Methodol, 7, 10. doi:10.1186/1471-2288-7-10

Tenzin, D. N., Rai BD, Changlo,Tenzin S, et al., Tsheten, K., Ugyen, P., Singye, K., Ward, M. P. 2012. Community-based study on knowledge, attitudes and perception of rabies in Gelephu, south-central Bhutan. Int Health, 4(3), 210-219. doi:10.1016/j.inhe.2012.03.005

Touihri L, Z. I., Elhili K, Dellagi K, Bahloul C, . 2011. Evaluation of Mass Vaccination Campaign Coverage Against Rabies in Dogs in Tunisia. Zoonoses and Public Health, 58(2), 110-118. doi:10.1111/j.1863-2378.2009.01306.x

Van Sittert SJ, R. J., Akol GW, Miyen JM. Mlahlwa B, et al., \& Sabeta, C. T. 2010. Rabies in the Eastern Cape Province of South Africa - Where are we going wrong? Journal of the South African Veterinary Association-Tydskrif Van Die Suid-Afrikaanse Veterinere Vereniging, 81(4), 207-215.

Wandeler AI, B. A., Capt S, Kappeler A, Matter H. 1988. Dog ecology and dog rabies control. Rev Infect Dis, 10 Suppl 4, S684-688.

Wera E, V. A., Geong M, Hogeveen H. (2013). Costs of rabies control: an economic calculation method applied to Flores Island. PLoS ONE, 8(12), e83654. doi:10.1371/journal.pone.0083654

WHO. 1998. Oral immunization of dogs against rabies. Report of the Sixth WHO Consultation, Geneva, 24-25 July 1995. Geneva. . WHO document WHO/EMC/ZDI/98.13.

WHO. 2013. World Health Organization- Rabies http://www.who.int/mediacentre/ factsheets/fs099/en/index.html. Updated July 2013, accessed date Nov 21, 2013.

WHO. 2005. World Health Organization expert consultation on rabies. . World Health Organization, Technical report series., 931, 1-88.

Yimer E, M. A., Beyene M, Bekele A, Taye G, et al., Zewdie, B., Alemayehu, T. 2012. Study on knowledge, attitude and dog ownership patterns related to rabies prevention and control in Addis Ababa, Ethiopia. Ethiopian Veterinary Journal, 16(2). doi:10.4314/evj.v16i2.3

Zinsstag, J. 2013. Towards a science of rabies elimination. Infectious diseases of poverty, 2(1), 22. 
Zinsstag J, D. S., Penny MA, Mindekem R, Roth F, et al., Menendez Gonzalez, S., Naissengar, S., \& Hattendorf, J. 2011. [Transmission dynamics and cost-effectiveness of rabies control in dogs and humans in an African city]. Médecine tropicale : revue du Corps de santé colonial, 71(6), 596-604.

Zinsstag, J., Dürr, S., Penny, M. A., Mindekem, R., Roth, F., Menendez Gonzalez, S., . . . Hattendorf, J. 2009. Transmission dynamics and economics of rabies control in dogs and humans in an African city. Proceedings of the National Academy of Sciences of the United States of America, 106(35), 14996-15001. doi:10.1073/pnas.0904740106

Supporting information: Checklist 2.1

PRISMA Checklist

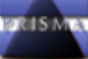

Checklist S1: PRISMA Check list Flow Diagram for the Systematic review of "Review on dog rabies vaccination coverage in Africa: A question of dog accessibility or cost recovery?"
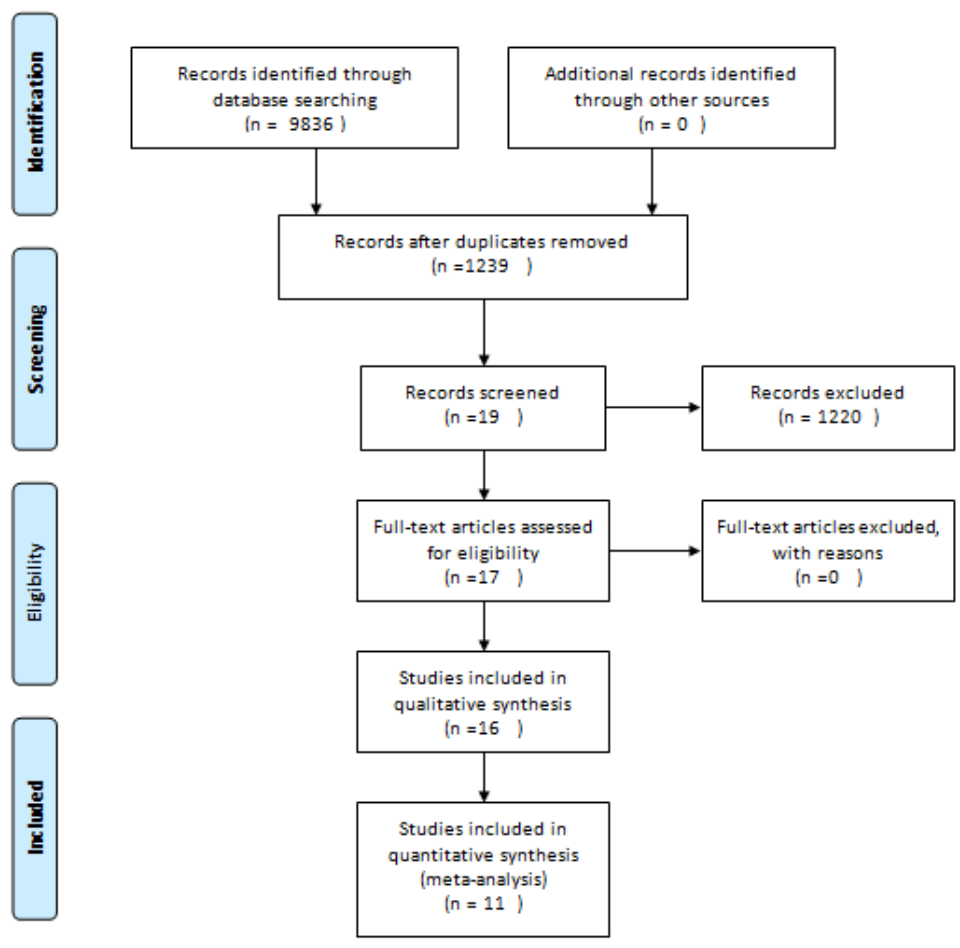

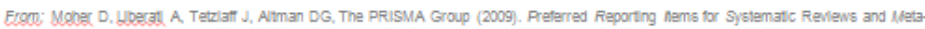
Analyses: The PRISMA Stalenent. RLQS Med 6(6): e1000097. do: 10.13711journal. pned 1000097 
Chapter 3

Dog rabies data reported to multinational organizations from Southern and Eastern African Countries

Tariku Jibat Beyene, Monique C.M. Mourits, Henk Hogeveen,

Published in BMC Research Notes 2017 10:199

https://doi.org/10.1186/s13104-017-2527-7 


\begin{abstract}
Rabies is one of the viral diseases with the highest case fatality rate in humans. The main transmission route to humans is through bites, especially of infected dogs. Decisions on the allocation of resources to control and reduce the socio-economic impacts of rabies require reliable data. Several national, regional and international organizations have been gathering rabies data for more than a decade. The objective of this paper was to examine the consistencies in the number of dog rabies cases reported to different multinational organizations by Southern and Eastern African Countries and to explore the presence of any time trend among the reported rabies data. Data was systematically extracted from the databases of the Southern and Eastern African Rabies Group - SEARG and the World Organization for Animal Health/World animal health information - OIE/WAHID. Despite differences in entities by which data have been reported to the two organisations, reported numbers were significantly correlated (Spearman's rho $=0.52, \mathrm{p}<0.001$ ). The reported data did not indicate the presence of any trend in the number of reported dog rabies outbreaks. Inconsistencies in the reported numbers were observed between the databases, possibly due to the fact that human and animal health authorities report separately to the organisations involved in addition to the use of indefinite definitions of report categories set by report receiving organizations. The observed report inconsistency underlines the need for collaboration within "One-health" approach between global and national authorities with respect to reliable data reporting, which is a necessity for a sound interpretation of developments within the disease status to implicate how the control of rabies need to be directed in the future.
\end{abstract}




\subsection{Introduction}

Rabies is one of the infectious diseases of humans and animals with almost hundred percent case fatalities (WHO, 2013). Annually more than 55,000 people die due to rabies globally with approximately $45 \%$ of these cases occurring in African countries. The main transmission route to humans is through animal bites, especially of infected dogs (Knobel et al., 2005).

Underreporting and data inconsistency are often mentioned as partial reasons for the lack of effective control and prevention of dog rabies in Africa (Cleaveland et al., 2002). Disease reporting is an essential component of monitoring and surveillance systems. Southern and Eastern African countries report on their rabies situation regularly to the SEARG (Southern and Eastern African Rabies Group) (SEARG, 2013) and to the OIE-World Animal Health Information Database (OIE, 2014). SEARG represents a group of independent researchers and public health officers of the various Southern and Eastern African countries and serves as a forum for gathering and disseminating rabies information. Available databases from regional, multi-national and international organizations need to be regularly evaluated for reasonable interpretation and possible implications on how the future rabies control needs to be directed. In previous studies like (Nel, 2013) the rabies data discrepancy have only been considered for a few African countries within a specific year (viz. 2007). Data reporting on dog rabies cases in the full region of Southern and Eastern African countries over a period of successive years has not been evaluated before. The objective of this paper was, therefore, to examine the consistencies in the number of dog rabies cases reported over a period of 8 years to the multinational organizations SEARG and OIE/WAHID by the Southern and Eastern African Countries and to explore the presence of any time trend among the reported rabies data. Such evidence based studies are potential inputs for devising regional rabies control strategies through improved information management.

\subsection{Materials and Methods}

Information of the occurrence of rabies was reported to SEARG and OIE/WAHID in different formats and entities. In SEARG records, the number of suspected and confirmed dog cases were registered with "suspected" referring to clinically diagnosed cases and "confirmed"' to cases tested positive for rabies by available laboratory tests. OIE/WAHID registers rabies outbreaks and defines an "outbreak" as the occurrence of one or more cases in an epidemiological unit (OIE, 2013). However, spatial and temporal specifications to distinguish one outbreak from another are not mentioned in the OIE/WAHID outbreak definition.

SEARG reports of 1996 till 2013 were examined on the number of rabies suspected and confirmed cases in dogs (SEARG, 2013). However, as many of the SEARG member countries did not report regularly during the indicated period, only countries with complete data reports 
between 2008 and 2012 were selected for further analysis. Subsequently, the respective annual reports of the selected countries from the OIE/WAHID - World Animal Health Information Database (OIE, 2013) were assessed to evaluate the number of reported outbreaks between 2005 and 2012.

The data from SEARG and OIE/WAHID were extracted from their online databases and stored in Microsoft Excel 2010. The stored data were analysed and presented using descriptive statistics (SPSS statistics 21). Data from the SEARG and OIE/WAHID datasets were compared to evaluate the extent of data registration and its consistency in registered numbers among the selected Southern and Eastern African countries. Consistency in the reported data to SEARG and OIE/WAHID was evaluated by means of a Spearman correlation test and a basic non parametric sign test to test the expectation that - in case of consistency - the confirmed number of cases reported to SEARG was at least equal or higher than the number of outbreaks reported to OIE/WAHID.

The presence of a trend in the number of dog rabies outbreaks was examined for each country by a linear regression on the number of outbreaks as reported to OIE/WAHID every 6 months during the period of 2005-2012. We also tested these data with a mixed effect model assuming country as random effect to evaluate the presence of an aggregated trend over time within the South East region of Africa. Due to the limited number of available data points from the SEARG database, trend analysis was only limited to the OIE/WAHID data.

\subsection{Results}

A total of 19 countries reported to SEARG between 1996 and 2013. Of these countries, only ten provided sustained data during the period of 2008-2012. These countries were South Africa, Botswana, Ethiopia, Kenya, Mozambique, Namibia, Tanzania, Uganda, Zambia and Zimbabwe. During the evaluated period, the highest number of reported cases and outbreaks was from South Africa while the lowest number was from Uganda. In countries like Zambia rabies was reported to SEARG as suspected and submitted for laboratory examination, while no outbreak was reported to OIE/WAHID (for example in 2011). On the other hand, no suspected case was reported to SEARG while about 500 outbreaks were reported to OIE/WAHID by South Africa in 2008 and 2009 (Table 3.1). This might be due to a lack of diagnostic facilities or inconsistency between reporting to SEARG and OIE. However, the number of suspected cases is higher than or the same as the number of confirmed cases (based on SEARG) and the number of outbreaks (based on OIE/WAHID) for all selected countries.

Spearman's rho between the numbers of confirmed cases (SEARG) and outbreaks (WHO/WAHID) as registered by the countries during 2008-2012 indicated a positive and significant correlation (Spearman's rho=0.52, $\mathrm{p}<0.001$ ). Consistency in the reported numbers 
of confirmed cases and outbreaks to SEARG and OIE evaluated by means of a non-parametric sign test revealed the $\mathrm{H} 0$ (median of difference between reported numbers $=0$ ) could not be rejected $(\mathrm{p}=0.312$ ), indicating the occurrence of inconsistency in the number reporting.

The linear regression analysis of number of outbreaks reported by OIE/WAHID revealed that there is no evidence of a significant increasing or decreasing trend in the number of rabies outbreaks ( $p>0.05$ ) in one of the countries. The mixed effect model also indicated that there was no indication of an increasing or decreasing trend within the complete South East African region (coefficient=0.08; $\mathrm{p}=0.11$ ) (Figure 3.1).

Table 3.1 Number of dog rabies suspected and confirmed cases as registered by SEARG and number of dog rabies outbreaks as registered by OIE/WAHID between 2008 and 2012 (SEARG suspected rabies cases / SEARG confirmed rabies cases)

\begin{tabular}{|c|c|c|c|c|c|c|c|c|c|c|}
\hline \multirow[t]{3}{*}{ Countries } & \multicolumn{10}{|c|}{ Year } \\
\hline & \multicolumn{2}{|c|}{2008} & \multicolumn{2}{|c|}{2009} & \multicolumn{2}{|c|}{2010} & \multicolumn{2}{|c|}{2011} & \multicolumn{2}{|c|}{2012} \\
\hline & SEARG & OIE & SEARG & OIE & SEARG & OIE & SEARG & OIE & SEARG & OIE \\
\hline Ethiopia & $67 / 46$ & 30 & $224 / 183$ & 38 & $278 / 206$ & 83 & $336 / 255$ & 34 & NA/NA & 50 \\
\hline Kenya & $63 / 54$ & 55 & $28 / 24$ & 63 & $32 / 30$ & 59 & $42 / 35$ & 79 & $61 / 50$ & 94 \\
\hline Mozambique & $41 / 31$ & 41 & $11 / 11$ & 5 & $78 / 11$ & 7 & $55 / 5$ & 2 & $53 / 10$ & 9 \\
\hline Botswana & $58 / 54$ & 67 & $65 / 32$ & 39 & $59 / 11$ & 29 & $71 / 32$ & 29 & $97 / 50$ & 46 \\
\hline Tanzania & NA/NA & 28 & NA/NA & 14 & $63 / 27$ & 16 & $71 / 36$ & 10 & $55 / 24$ & 3 \\
\hline Namibia & $125 / 51$ & 32 & 198/68 & 90 & $184 / 138$ & 175 & $183 / 57$ & 207 & $179 / 50$ & 245 \\
\hline South Africa & NA/NA & 488 & NA/NA & 522 & $1069 / 409$ & 401 & $912 / 331$ & 295 & $1186 / 508$ & 426 \\
\hline Zimbabwe & $84 / 27$ & 61 & $27 / 11$ & 38 & $116 / 65$ & 138 & $169 / 134$ & 184 & $104 / 59$ & 160 \\
\hline Uganda & NA/NA & NA & NA/NA & NA & $4 / 3$ & 1 & $9 / 9$ & 3 & $9 / 6$ & 4 \\
\hline Zambia & $46 / 16$ & 106 & $39 / 17$ & 125 & $62 / 46$ & 96 & $51 / 36$ & NA & $42 / 24$ & 40 \\
\hline
\end{tabular}




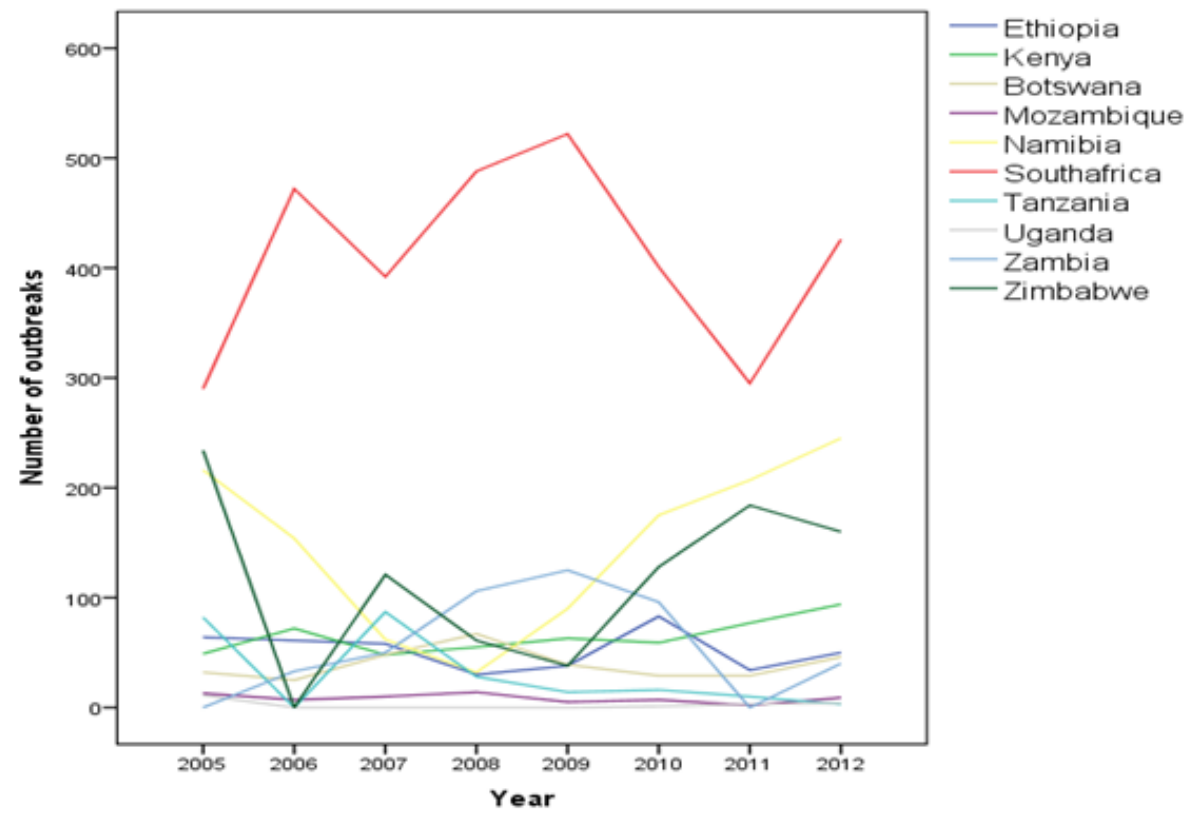

Figure 3.1 Trend of number of rabies outbreaks in Southern and Eastern Africa countries from 2005 through 2012 based on OIE/WAHID.

\subsection{Discussion}

Despite the differences in entities by which the data on dog rabies incidences have been reported by SEARG and OIE/WAHID, the positive correlation between the registered data indicates that both systems would be able to signal changes in number of occurrences between succeeding years. Given the half yearly OIE data of 2008-2012 there was, however, no evidence of an increasing or decreasing dog rabies trend in the evaluated Southern and Eastern African countries.

Due to entity differences as well as the limited SEARG data set size, the occurrence of inconsistency in the reported numbers was only tested indirectly by means of a non-parametric sign test. After all, one reported outbreak in OIE should at least correspond to one reported confirmed SEARG case, resulting in the hypothesis that the number of reported confirmed cases in SEARG should be equal or larger than the number of registered outbreaks in OIE. The test showed that this hypothesis is not supported by the registered data, indicating the occurrence of inconsistency in the reported numbers when comparing the data entries of both data sets.

The inconsistency might be due to the process of separate reporting by the countries to regional and international organizations as well as to a lack of collaboration between reporting national 
human health and veterinary sectors (Nel, 2013). For instance in Ethiopia, the veterinary department, which is under the Ministry of Agriculture, reports to OIE, while the Ethiopian Institute of Public Health, which is under the Ministry of Health, reports to SEARG about rabies without consulting each other or cross checking the reported data (personal communication Dr Assefa Deressa). With the reporting hierarchy of Ethiopia reflecting the general structure of most reporting hierarchies, similar reasons could be valid for the other African countries. Moreover, the ambiguous definitions on the terms of "suspected" cases, "confirmed" cases and "outbreaks", and the lack of enforcing compulsory reporting will have contributed to the inconsistency as well.

As such the comparison of registered data between the two data bases does not provide insight in the extent of underreporting as neither of the databases can be considered as a golden standard. According to a recent study on the global disease burden of 2010 (Hampson et al., 2015) human fatalities due to rabies were expected to occur in each of the 10 evaluated countries. Estimated human case fatalities (n) equalled in this study to 2771 in Ethiopia, 523 in Kenya, 1326 in Mozambique, 3 in Botswana, 345 in Tanzania, 4 in Namibia, 42 in South Africa, 410 in Zimbabwe, 133 in Uganda and 48 in Zambia. With domestic dogs as the main transmitters of the rabies virus to human (Knobel et al., 2005), some serious underreporting is expected when comparing the number of registered cases and outbreaks with the indicated number of estimated human casualties. For instance, Uganda reported for 2010 only 3 confirmed dog cases and 1 outbreak, while the global rabies burden estimated 133 human deaths (Hampson et al., 2015; Fevre et al., 2005).

The limitations of this study also extend to the unsuitability of the datasets to apply better methods of dataset comparison over a period of time, like autoregressive models for time series, and application of robust statistical tests. This is because the OIE/WAHID datasets we used for this study have a limited number of entries. Moreover, they are reported by human health and veterinary authorities in each country who practice different reporting structures as mentioned earlier for the case of Ethiopia. Reporting structures might even have been changed within a country over time. As a consequence, the time series are relatively short and the data points are difficult to compare between countries. Since robust statistical tests could not be performed, the findings from this study should be interpreted with caution. Finally, there is no similar study published. This means that we could not triangulate our findings with other findings.

The emerging approach of controlling zoonotic diseases like rabies is through an coordinated effort of animal and human health authorities or by the so-called One Health approach (Zinsstag et al., 2005). An example of such an approach is the Zoonotic Disease Unit of Kenya, which is organized by the Ministry of Livestock Development together with the Ministry of Public Health and Sanitation established to improve prevention and control of diseases transmissible 
between animals and human. This approach strengthens the One Health concept by maturing the collaboration and synergy between human health and veterinary officers. With this synergy it is possible to identify and deal across sectoral issues which reduces redundancy and increases efficiency of resource utilization with better outcomes in terms of disease information and burden reduction. In addition, reporting disease information to a single national body from which other regional or international organizations obtain their information could prevent the occurrence of discrepancies and deficiencies in rabies reports (Nel, 2013).

\subsection{Conclusion}

Although the regional organisation SEARG and the international organisation OIE collect their information on rabies occurrence in different entities, numbers on reported rabies cases (SEARG) and outbreaks (OIE) were significantly correlated. Reported data did not provide any evidence of an increasing or decreasing of any trend in rabies occurrence in Southern and Eastern African countries. Inconsistencies between the reported numbers in the two databases were observed, while underreporting in general is expected. The hierarchic reporting system, lack of enforcing bodies for compulsory reporting and ambiguous definition for the report heading terms might have contributed to these discrepancies in rabies data. A strict application and enforcement of the One Health concept by animal health and human health authorities working on a common understanding is potentially a solution for better data reporting and further utilization in the future direction of rabies control. Furthermore, there is a need for an improved collaborative effort and effective communication between all relevant authorities.

\section{List of abbreviations}

SEARG- Southern and Eastern African Rabies Group

OIE/WAHID- World Organization for Animal Health/World animal health information

\section{Acknowledgements}

The authors would like to acknowledge SEARG- Southern and Eastern African Rabies Group for availing and permission to use the data while OIE/WAHID- World Organization for Animal Health/World animal health information for availing the data online. 


\subsection{References}

Cleaveland, S., Fevre, E. M., Kaare, M., Coleman, P. G. 2002. Estimating human rabies mortality in the United Republic of Tanzania from dog bite injuries. Bulletin of the World Health Organization, 80(4), 304-310.

Fevre, E. M., Kaboyo, R. W., Persson, V., Edelsten, M., Coleman, P. G., Cleaveland, S. 2005. The epidemiology of animal bite injuries in Uganda and projections of the burden of rabies. Tropical Medicine and International Health, 10(8), 790-798. doi:10.1111/j.1365-3156.2005.01447.x

Hampson, K., Coudeville, L., Lembo, T., Sambo, M., Kieffer, A., Attlan, M., . . . on behalf of the Global Alliance for Rabies Control Partners for Rabies, P. 2015. Estimating the Global Burden of Endemic Canine Rabies. PLoS Negl Trop Dis, 9(4), e0003709. doi:10.1371/journal.pntd.0003709

Knobel, D. L., Cleaveland, S., Coleman, P. G., Fevre, E. M., Meltzer, M. I., Miranda, M. E., . . . Meslin, F. X. 2005. Re-evaluating the burden of rabies in Africa and Asia. Bulletin of the World Health Organization, 83(5), 360-368. doi:/S0042-96862005000500012

Nel, L. H. 2013. Discrepancies in data reporting for rabies, Africa. Emerging Infectious Diseases, 19(4), 529-533. doi:10.3201/eid1904.120185

OIE. 2013. Terrestrial Animal Health Code. Volume I. General provision. http://web.oie.int/eng/normes/mcode/en_glossaire.htm.

OIE. 2014. http://www.oie.int/wahis_2/public/wahid.php/Diseaseinformation/statusdetail. Date accessed January 07, 2014.

SEARG. 2013. http://www.searg.info/doku.php, Date accessed December 20, 2013. .

WHO. 2013. World Health Organization- Rabies http://www.who.int/mediacentre/ factsheets/fs099/en/index.html. Updated July 2013, accessed date Nov 21, 2013.

Zinsstag, J., Schelling, E., Wyss, K., \& Mahamat, M. B. 2005. Potential of cooperation between human and animal health to strengthen health systems. The Lancet, 366(9503), 21422145. 



\section{Chapter 4}

\section{Incidence and economic impacts of rabies in the cattle population of Ethiopia}

Tariku Jibat Beyene, Monique C. M. Mourits, Henk Hogeveen

Published in Preventive Veterinary Medicine 130 (2016) 67-76

http://dx.doi.org/10.1016/j.prevetmed.2016.06.005 


\begin{abstract}
Rabies is a viral disease that can cause fatal encephalomyelitis both in animals and humans. Although incidences of the disease in cattle have been reported, insight in the economic impact of the disease in livestock remains limited. By affecting cattle in subsistence systems, rabies may have extensive economic impacts at household and country levels, in addition to the effects on human health. This study presents estimates of the direct economic impact of rabies at herd level in two representative subsistence cattle-farming systems in Ethiopia, the mixed croplivestock and pastoral production systems. The economic impacts were assessed by a structured questionnaire administered to 532 cattle-owning households. These households were selected from four districts within two administrative zones; each zone representing a cattle production system. Rabies incidence rates of $21 \%$ and $11 \%$ at herd level were calculated for the mixed crop-livestock and pastoral production systems, respectively. The incidence rate at cattle level was the same in both systems, i.e. $2 \%$. Herd-level incidence rates were higher in the mixed crop-livestock system than in the pastoral system $(\mathrm{P}<0.05)$. Average economic losses per herd due to rabies were estimated at 49 USD per year for the mixed-crop livestock system, and at 52 USD per year for the pastoral system; whereas in affected herds the average losses per year were 228 USD (range 48-1016 USD) in the mixed crop-livestock system, and 477 USD (range 173-1140 USD) in the pastoral system. The average herd-level economic losses were not significantly different between the farming systems; however once the herd was affected, the losses were significantly higher for the pastoral system than for the mixed crop-livestock system $(\mathrm{P}<0.01)$. The losses due to rabies in cattle are relatively high for pastoral households, due to their complete dependency on livestock for their livelihoods. Although the current estimates only account for the direct losses resulting from cattle mortality, the estimates already indicate the potential economic gains from a rabies intervention in the dog population, of which the benefits can be shared by the public health sector.
\end{abstract}




\subsection{Introduction}

Rabies is a viral disease that causes fatal encephalomyelitis in animals and humans (Wunner and Conzelmann, 2013). A recent global estimate indicates that rabies is annually responsible for 60,000 human death cases (Hampson et al., 2015). Most of these cases occur in Asian and African countries (Knobel et al., 2005; Hampson et al., 2015), where domestic dogs are the main transmitters of the rabies virus (Knobel et al., 2005).

In Africa, rabies has also been reported as a potential problem for cattle production in freerange production systems, such as the mixed crop-livestock and pastoral production systems. In these systems dogs are kept in close contact with cattle (Sillero-Zubiri and Switzer, 2004), providing an opportunity to transmit the virus to cattle through a bite of an infected dog. By affecting cattle in subsistence systems, rabies may have extensive economic impacts at the household and country levels, in addition to the effects on human health (Okell et al., 2013). Rabies in cattle causes direct losses due to mortality and reduced milk yield. It affects the livelihoods of people through lower food security, due to the loss of protein resources and draught power (Tambi et al., 2006). Worldwide, only a few studies have estimated the economic impact of rabies in livestock (Hampson et al., 2015). These estimates were made at the aggregated level of continent or region and did not explain the impact at individual country level.

A large dog population size in combination with poor dog management has resulted in a high endemicity of canine rabies in Ethiopia, accounting for an estimated death rate of 10,000 people a year (Jemberu et al., 2013). In Ethiopia many households own dogs usually for herding livestock or guarding property. Statistics on the dog population size is lacking, but a conservative estimation indicates an average of one owned dog per five households (Deressa et al., 2010). Available studies on the incidence of rabies in the dog population indicate rates as high as 413 cases per 100,000 in dogs (Jemberu et al., 2013). Currently, there is no official rabies control program in Ethiopia. Dog vaccination coverage is very low due to the limited availability of vaccines, lack of awareness, and the perception of high vaccination costs (Ali et al., 2010; Jibat et al, 2015).

With 57 million heads of cattle, Ethiopia owns the largest cattle population in Africa (CSA, 2012). Occurrence of rabies in cattle has been reported in central and northern parts of the country (Ali et al., 2010; Jemberu et al., 2013). As the livestock sector in Ethiopia provides livelihoods to $65 \%$ of the human population (Solomon, 2003) occurrence of rabies in cattle can have serious economic consequences. Accurate information on the incidences of rabies in humans, dogs and livestock is, however, scarce in Ethiopia (Jemberu et al., 2013), due to the absence of proper registration systems. Rabies-related medical and veterinary records are 
incomplete and cannot be used for a proper quantification of the impact of rabies (Tefera et al., 2002; Adane et al., 2013; EHNRI, 2012; Randall et al, 2004).

Representative information on the economic impact of rabies in livestock needs, therefore, to be collected from livestock owners directly, using participatory and conventional survey approaches. Quantitative information on the impact of rabies in livestock can support decisions about rabies control and strengthen the justification for the implementation of a one-health approach to fight diseases in resource-limited countries, such as Ethiopia (Zinsstag et al., 2011). In this study, we aim to assess the direct economic impact of rabies in cattle in the subsistence oriented farming systems of Ethiopia, using representative and quantitative data obtained through participatory and conventional survey approaches.

\subsection{Materials and methods}

\subsubsection{Study site selection}

In Ethiopia, cattle production takes primarily place in subsistence farming systems, i.e. the mixed crop-livestock system and the pastoral system (Tolera and Abebe, 2007; Alemayehu, 2011). The mixed crop-livestock system prevails in the central highland parts of the country and accounts for about $80-85 \%$ of the cattle population (MoARD, 2007). In this system, cattle provide draft power for the crop production as well as manure for fertilizer and cooking fuel, and meat and milk for household consumption. The pastoral system is practised in the arid and semiarid peripheral parts of the country and accounts for $15-20 \%$ of the population (MoARD, 2007). In this system, cattle farming is the main livelihood providing farm cash income as well as food for subsistence.

The study was conducted in the administrative zones of Arsi and Borena (Figure 4.1). These zones were selected for their representation of the two subsistence farming systems. Arsi represents the mixed crop-livestock production system and is located in the central highlands about $150 \mathrm{~km}$ east of Addis Ababa. People in this zone live predominantly from crop production. Borena represents the pastoral production system and is located in the southern lowlands about $600 \mathrm{~km}$ south of Addis Ababa. This zone predominantly produces livestock, as crop production is not feasible given the shortage of rainfall throughout the year.

\subsubsection{Study design}

A multistage cluster sampling technique was used to select representative districts and villages from both zones. Selection for the first cluster (district level) was done purposely by selecting the districts with the largest livestock populations. For Arsi these districts were Munessa (population size $=242,400$ ) and Lemuna-Bilbilo (population size $=232,949$ ), for Borena these 
districts were Yabelo (population size = 232,949) and Dugda Dawa (population size = 294,202). Selection for the next cluster (village) was done randomly within the district. Five villages per district were chosen based on a pragmatic consideration of logistic feasibility. Accordingly, 20 villages were selected in total, resulting in a sample size of 532 livestock-owning households; 248 in the mixed crop-livestock system and 284 in the pastoral system.

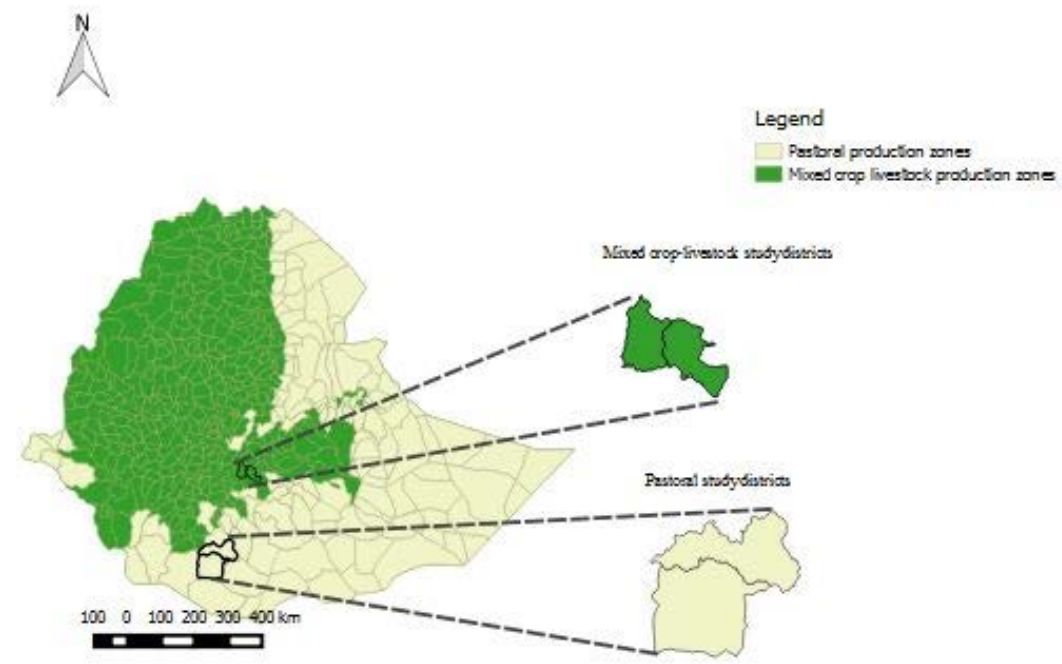

Figure 4.1 Map of Ethiopia showing major livestock production systems and the study districts.

\section{General setup}

We first performed a preliminary exploration using a participatory approach, because of uncertainty about the presence of rabies in the selected areas and about the ability of livestock owners to identify the disease among their livestock. The preliminary exploration was undertaken by means of a group discussion with elder livestock owners in each of the 20 selected villages. This was followed by interviews with all livestock-owning households $(\mathrm{N}=532)$ in the selected villages, using a structured questionnaire to collect data to assess the direct economic impact of rabies in the mixed crop-livestock and pastoral systems.

\section{Preliminary exploration of rabies in cattle}

In each of the selected villages, we asked the village administrators to identify 15 elder livestock owners who had lived in the village for more than 20 years and were older than 50 years of age. We approached these elders to participate in a group discussion on zoonotic diseases. As a 
group size of 10 participants is accepted practice in participatory appraisal (Catley et al., 2002), the group discussions were joined by 10-12 livestock owners in each of the selected villages.

The group discussion was conducted in the local language (Oromifa), which all participants understood. Local facilitators were available to communicate in other local languages if needed, to ensure equal understanding. The discussions were recorded using a voice recorder and transcribed at a later date.

Each group was asked to identify and list the local names of zoonotic diseases that are transmitted to cattle and humans and which have occurred in their area recently during the past five years. Neither the livestock owners nor the local facilitators were informed that the main disease of interest was rabies, to minimise response bias. The group discussions were structured so that participants could easily share their opinions with the group, in an environment that encouraged everyone's participation to reach a final consensus on the list of zoonotic diseases (Catley et al., 2002).

Once the participants had listed the local names of recently (during the past five years) occurring zoonotic diseases, they were asked to describe the clinical signs of these diseases in cattle, which they either knew or had experienced. Subsequently, the group was asked to rank these diseases according to five criteria, using the procedure described by (Okell et al., 2013). The criteria were: 1) the perceived risk of the disease in terms of susceptibility and severity to human health, 2) the perceived possibility to treat and 3) prevent the disease in cattle, 4) the perceived mortality rate in cattle, and 5) the perceived morbidity rate in cattle. To identify the English name of each disease, we compared the description of clinical signs given by the participants with the description of a particular disease in the Merck veterinary manual (Kabeta et al., 2015; Kahn et al., 2010).

\section{Structured questionnaire on rabies in cattle}

Once the preliminary exploration indicated the presence of rabies in cattle in a village, each livestock-owning household in the village was interviewed to assess the extent to which the household's cattle were affected by rabies or bitten by suspected rabid dogs during the past year (September 2013 to August 2014).

For this purpose, a structured questionnaire was developed in English and translated to the local language, Oromifa, in which it was administrated. The Oromifa version was translated back to English to check consistency. People who assisted the respondents while filling the questionnaire were trained in assisting to obtain a uniform type of response. A pilot of the questionnaire was pretested on ten households in each zone. Based on the results of this pilot, minor modifications were made to match the dialects of Oromifa in the two zones. 
All livestock-owning households in the selected villages (100\%) were interviewed to assess the extent to which the household's cattle were affected by rabies or bitten by suspected rabid dogs during the past year (September 2013 to August 2014). In Ethiopia, cattle are generally slaughtered when bitten by a suspected rabid dog, as owners do not want to face the risk that their cattle will develop clinical signs. For this reason households were explicitly asked about their experiences in affected cattle and exposed (bitten by a suspected dog) cattle.

The questionnaire started with a general question about the experience of rabies in the households' cattle during the last year, followed by specific questions on the cattle that were considered to be affected by rabies and/or bitten by a suspected rabid dog. These questions covered the gender and estimated age of the cattle, the clinical symptoms of suspected rabid dogs in cases where cattle were bitten, the amount of money received for 'sale' of meat from cattle bitten by a suspected dog and the clinical signs observed in cattle in the case of an unknown source of exposure. See for an overview of the questionnaire Supplementary information 4.1 .

\section{Cattle exposed to a known suspected rabid dog}

If the livestock owner experienced his cattle to have been exposed to a known, suspected rabid dog, then the rabies status of the dog was evaluated based on the six criteria for rabies diagnosis in living dogs, as described by Tepsumethanon et al. (2005) (Figure 4.2). If the dog was judged to be rabid, then the bitten cattle were considered as exposed to rabies.

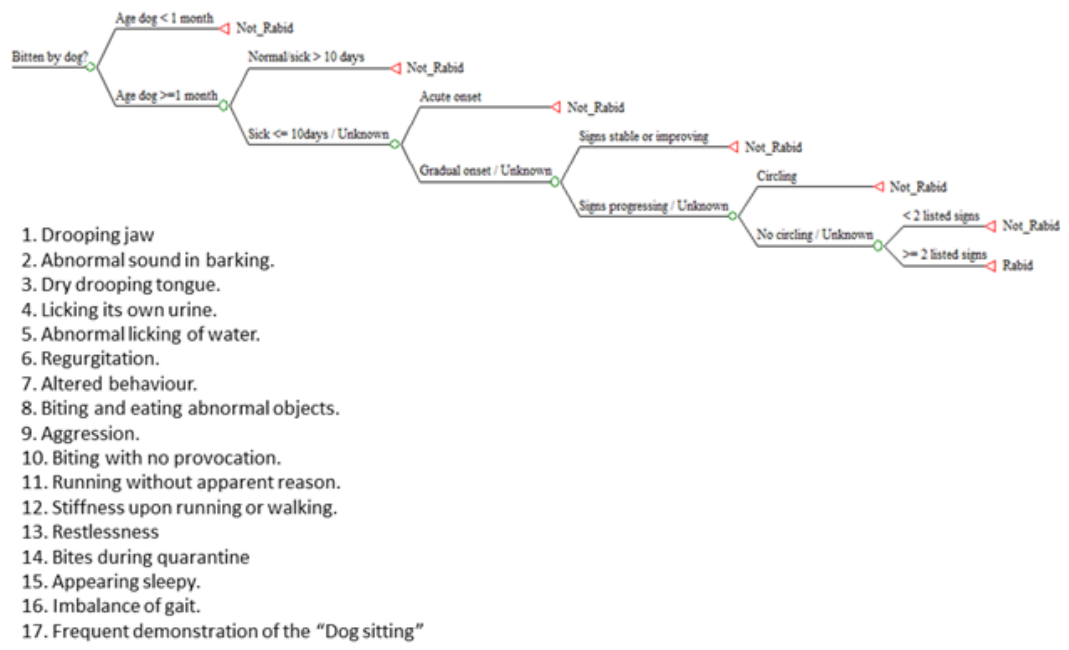

Figure 4.2 Decision tree based on the six criteria of Tepsumethanon et al. (2005) to retrospectively assess the rabies status of a biting dog. The final criterion in the decision tree refers to the list of 17 clinical signs at the left of the figure. 


\section{Cattle showing clinical signs of rabies without known exposure}

If the livestock owner considered that his cattle exhibited neurological signs without knowing whether the cattle were exposed to a suspected rabid dog, then the evaluation of the rabies status of the cattle was done by comparing the clinical signs described by the livestock owner with the clinical signs of rabies as described in scientific literature (Kahn et al., 2010) and summarized in Supplementary information 4.2. To verify the cattle-owner's statements, we asked the neighbours if they had seen or heard that the cattle were sick from rabies or bitten by a suspected rabid dog. In Ethiopia, most households in neighbourhoods are socially close to each other (Fafchamps et al., 2009), and are therefore well informed about events happening to households in the village. As cattle are important, cattle losses due to disease or any other cause are well known in the neighbourhood.

\subsubsection{Statistical analyses}

\section{Analysis of group discussion}

The group discussions captured by voice recorder were transcribed and recorded in Microsoft Excel 2007. The relative importance of zoonotic diseases was assessed by ranking the diseases for each evaluated criterion, the first rank reflecting the highest importance. For ease of interpretation, the ranks were converted to standard scores as described by Okell et al. (2013). The ranks given to each disease for each criterion were converted to scores so that the lowest rank (reflecting the highest importance) received the highest score (Equation 4.1). When a group did not identify a disease, a score of zero was assigned. As the number of diseases identified by each group varied, a further standardisation was made (Equation 4.2).

$$
\begin{aligned}
& S_{i j k}=N_{i}-R_{i j k}+1 \\
& \text { Equation } 1
\end{aligned}
$$

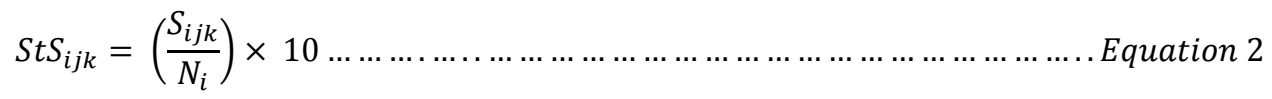

Where, $S_{i j k^{-}}$the score given by the $i^{\text {th }}$ group for the $j^{\text {th }}$ disease for criterion $k$;

$N_{i}$ - the number of diseases identified by the $i^{\text {th }}$ group;

$R_{i j k}$-the rank given to disease $j$ by group $i$ for criterion $k$;

$S t S_{i j k}$-the standardised score for the $j^{\text {th }}$ disease by the $i^{\text {th }}$ group for criterion $k$.

Due to the non-parametric nature of the data collected, median values of the standardised scores for each criterion were used to compute the Kruskal Wallis pairwise comparison test to evaluate the relative importance of rabies compared to the other identified zoonotic diseases. 


\section{Analysis of questionnaire survey}

\section{Estimation of incidence rate}

The incidence rate at herd level was calculated per village by dividing the number of herds affected by the number of herds studied. In this study, herd is defined as a group of cattle that belong to a household. The incidence rate at individual cattle level was calculated for each village by dividing the total number of cattle affected by the total number of cattle at risk.

The number of cattle affected per herd was obtained from the data collected during the survey. However, reliable information on individual herd sizes was lacking. During the preliminary group discussion, we were informed that people would not be willing to tell the exact total number of cattle they own in fear of local taxes. We could not guarantee the anonymity of our registration as we interviewed every household in the village. Therefore, we calculated individual cattle incidence rates at village level and not at individual herd level (personal communication with local animal health workers and district officers) by using livestock population data as registered at village level by district agricultural offices for the year 20132014. We also used the registered number of other livestock species to get the average number of livestock per herd at village level for comparison with the number of cattle kept in each herd.

\section{Estimation of economic loss due to occurrence of rabies in livestock}

In Ethiopia, cattle that are considered to be affected by rabies and/or bitten by a suspected rabid dog are killed and/or slaughtered. The estimation of direct economic losses due to the occurrence of rabies in livestock was, therefore, associated with the number of exposed cattle that died as a consequence of these rabies incidence related events, reflected by the surveyed mortality in cattle.

Cattle bitten by a suspected rabid dog do not per definition develop rabies. In practice, however, these animals are slaughtered as a precautionary measure, as owners do not want to face the risk that their cattle gradually develop clinical signs. Instead, they slaughter the animals and sell the meat to their neighbours. In this way, cattle bitten by a suspected rabid dog have some salvage value. In contrast, cattle that are considered to be rabid have no salvage value, as they are killed and buried by the owners. Thus, all exposed cases ended up dead as a result of the disease (if unnoticed when exposed) or due to the precautionary slaughter. The estimation of the direct losses was conducted for the average herd and the average affected herd in a village. 
Mathematically

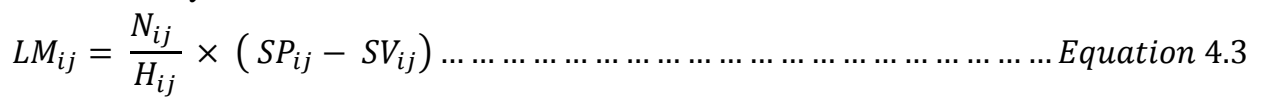

Where:

$L M_{i j}$ - Loss due to mortality per herd in village $i$ of district $j$;

$N_{i j}$ - Total number of cattle affected by rabies in village $i$ of district $j$;

$H_{i j}$ - Number of herds studied in village $i$ of district $j$;

$S P_{i j}$ - Average sales price of live cattle in village $i$ of district $j$;

$S V_{i j}$ - Average salvage value of the meat and hides in village $i$ of district $j$.

Thus, the economic losses due to cattle mortality per herd were calculated by dividing the number of affected cattle within a village by the number of herds at risk, multiplied by the difference between the average sales value and average salvage value of cattle. The actual losses due to mortality were estimated as the difference between the market price and the salvage value of the animal.

The market prices of cattle at different ages were obtained from weekly records of each district's revenue office, collected over the period from September 2013 to August 2014. From this data, the average cattle sales value was estimated to be 248 USD in the districts representing the mixed crop-livestock system and 280 USD in the districts representing the pastoral production system. Data on the salvage value of the affected cattle with known exposure was obtained during the survey by asking the amount of money the owner collected from the meat 'sale'. The average salvage value of these animals was 81 USD for the districts representing the mixed crop-livestock system and 77 USD for the pastoral system districts. We assumed a salvage value of zero for cattle considered to be rabid with unknown exposure.

We tested if there were differences in herd-level economic losses from rabies between farming systems and between districts within farming systems. A chi-square test was used to evaluate the differences in herd-level and cattle-level incidence rates between farming systems and between districts within farming systems. As the economic losses were not normally distributed (Shapiro and Wilk, 1965; Razali and Wah, 2011), we used a Kruskal-Wallis pairwise comparison test to compare the differences in herd-level economic losses between the two farming systems. The non-parametric tests and comparison of economic losses per herd were performed using IBM SPSS statistical package Version 21, (IBM corp., USA). 


\subsection{Results}

\subsubsection{Characteristics of herds within the studied villages}

The average size and composition of the livestock herds in each district are presented in Table 4.1. There is no statistical difference $(p>0.05)$ in the mean number of cattle per herd between the farming systems. The mean number of cattle per herd is 13.0 in the mixed crop-livestock system and 15.5 in the pastoral system. Cattle constitute the largest proportion of the livestock herds in both farming systems. Camels are absent and goats are rare in the mixed crop-livestock system, whereas horses and donkeys are rare in the pastoral system.

Table 4.1 Average size and composition of the livestock per herd in each district and farming system as derived from livestock population data registered at village level (= data on 5 villages per district) by district agricultural offices for the year 2013-2014 and the number of surveyed herds per village (Munessa 119 herds, Lemuna-Bilbolo 129 herds, Yabelo 134 herds and Dugda Dawa 150 herds). Range indicates the variation among the 10 villages studied in each system.

\begin{tabular}{|c|c|c|c|c|c|c|c|c|}
\hline & \multicolumn{4}{|c|}{ Mixed crop-livestock farming system } & \multicolumn{4}{|c|}{ Pastoral farming system } \\
\hline & \multicolumn{2}{|l|}{ District } & \multirow[t]{2}{*}{ Mean } & \multirow[t]{2}{*}{ Range } & \multicolumn{2}{|l|}{ District } & \multirow[t]{2}{*}{ Mean } & \multirow[t]{2}{*}{ Range } \\
\hline & Munessa & $\begin{array}{l}\text { Lemuna- } \\
\text { Bilbilo }\end{array}$ & & & Yabelo & $\begin{array}{l}\text { Dugda } \\
\text { Dawa }\end{array}$ & & \\
\hline Cattle & 11.3 & 14.5 & 13.0 & $6-21$ & 16.2 & 15.5 & 15.5 & 6-48 \\
\hline Camel & 0.0 & 0.0 & 0.0 & $0-0$ & 3.0 & 2.4 & 3.0 & $0-8$ \\
\hline Goat & 0.5 & 1.0 & 0.7 & $0-2$ & 11.9 & 10.2 & 11.0 & $4-29$ \\
\hline Sheep & 7.8 & 14.5 & 11.0 & $6-25$ & 5.7 & 4.5 & 5.0 & $0-13$ \\
\hline Horse & 1.8 & 2.4 & 2.0 & $0-5$ & 0.0 & 0.0 & 0.0 & $0-1$ \\
\hline Donkey & 1.3 & 1.2 & 1.2 & $0-2$ & 0.4 & 0.9 & 0.5 & $0-3$ \\
\hline
\end{tabular}

\subsubsection{Relative importance of rabies}

Based on the results of the 20 group discussions, a total of nine zoonotic diseases were identified that could spread from animals to humans: Rabies, Anthrax, Brucellosis, Tuberculosis, Teania, Mange mite, Wart, "Liver disease" and "Milk contaminant". We were unable to find the direct English names for the latter two diseases. The elder livestock owners described "Liver disease" as being transmitted from cattle to humans when the meat is consumed raw from cattle whose liver is diseased and yellow. "Milk contaminant" was described as a sickness occurring when raw milk is consumed, and which is sometimes related to milk obtained from cows with udder or milk abnormalities. Rabies was the only zoonotic disease identified by all of the 20 groups; it was therefore clearly occurring and being recognised in all of the selected villages. Anthrax was identified by 19 groups, followed by Tuberculosis (9), Teania (8), Brucellosis (6), Mange mite (4), Wart (3), "Liver disease” (3) and "Milk contaminant” (2) (Table 4.2). The diseases were identified in both farming systems, except "Liver disease” and "Milk contaminant”, which were mentioned only in the pastoral discussion groups. Rabies was ranked the highest with respect to the perceived risk to human health and the mortality and morbidity rates in cattle, 
followed by Anthrax. Anthrax was ranked the highest in terms of possibility to treat and possibility to prevent, followed by rabies.

The pairwise comparison test indicates that rabies was ranked significantly different in terms of mortality rate in cattle, showing that rabies is perceived as the most fatal disease in cattle compared to the other zoonotic diseases identified. It was also ranked differently ( $p<0.01$ ) from the other zoonotic diseases in terms of the perceived risk to human health, with the exception of Anthrax.

\subsubsection{Incidence rates of rabies in cattle}

The total population of households in the selected villages participated in the survey, as every household had at least one head of cattle and no households refused to participate in the survey. Of the 248 herds surveyed in the mixed crop-livestock system, 52 experienced rabies in cattle, with a total of 72 affected cattle. In the pastoral system, 31 of the 284 herds surveyed were affected by rabies during 2013-2014, resulting in a total of 73 affected cattle. The rabies incidence rates at herd level and cattle level for each village, calculated from the structured questionnaire, are presented in Figure 4.3.

The average herd-level incidence rate per village was $20.9 \%$ in the mixed crop-livestock system and $10.9 \%$ in the pastoral system; the overall herd-level incidence rate per village was $15.6 \%$ (Table 4.3). The herd-level incidence rates per village were higher $(\mathrm{p}<0.05)$ in the mixed croplivestock system than in the pastoral system. The cattle-level incidence rates within a village were $2.2 \%$ in the mixed crop-livestock system and $1.7 \%$ in the pastoral system. The overall cattle-level incidence rate per village was $1.9 \%$. Cattle-level incidence rates per village were not statistically different $(p>0.05$ ) between the farming systems. The comparison of the districts within a farming system revealed that there was a significant difference in terms of herd-level and cattle-level incidence rates per village between the districts of the pastoral farming system.

Of the 72 cattle cases in the mixed crop-livestock farming system, $16.7 \%$ were exposed to rabies from an unknown source (e.g. the livestock owner was not aware of any bite incident), resulting in actual affected cattle cases. Results for the pastoral farming system were similar; $16.4 \%(12 / 73)$ of the cattle cases were exposed to an unknown source. All of the clinical signs in cattle and suspected rabid dogs, which were described by the owners, were consistent with the clinical signs in cattle as described in the Merck veterinary manual (Kahn et al., 2010) and the six criteria for rabies diagnosis in living dogs (Tepsumethanon et al., 2005). The cross-check we performed to assess the validity of the data obtained from livestock owners confirmed the data provided by owners; for all rabies cases we were able to find at least one neighbour who confirmed the owner's statements. 
Table 4.2 Identified zoonotic diseases, standardised scores per criterion and significant differences in the ranking of rabies compared to the other identified diseases (number of groups $=20$ )

\begin{tabular}{|c|c|c|c|c|}
\hline Criteria & Disease & $\begin{array}{l}\text { Number of groups } \\
\text { identifying the disease }\end{array}$ & $\begin{array}{c}\text { Standardised } \\
\text { median score (min*, } \\
\text { max) }\end{array}$ & $\mathbf{P}$ value $* *$ \\
\hline \multirow{9}{*}{$\begin{array}{l}\text { Perceived risk to human } \\
\text { health }\end{array}$} & Rabies & 20 & $10(6.7-10)$ & $\mathrm{Na}$ \\
\hline & Anthrax & 19 & $7.1(0-10)$ & 0.2 \\
\hline & Brucellosis & 6 & $0(0-6)$ & $<0.01$ \\
\hline & $\mathrm{TB}$ & 9 & $0(0-6)$ & $<0.01$ \\
\hline & Teania & 8 & $0(0-5)$ & $<0.01$ \\
\hline & Mange mite & 4 & $0(0-4)$ & $<0.01$ \\
\hline & Wart & 3 & $0(0-6)$ & $<0.01$ \\
\hline & "Liver disease" & 3 & $0(0-10)$ & $<0.01$ \\
\hline & “Milk contaminant” & 2 & $0(0-6)$ & $<0.01$ \\
\hline \multirow[t]{9}{*}{ Possibility to treat locally } & Rabies & 20 & $3.3(2-6.7)$ & $\mathrm{Na}$ \\
\hline & Anthrax & 19 & $6.7(0-10)$ & $<0.01$ \\
\hline & Brucellosis & 6 & $0(0-10)$ & 0.06 \\
\hline & $\mathrm{TB}$ & 9 & $0(0-10)$ & 0.21 \\
\hline & Teania & 8 & $0(0-10)$ & 0.20 \\
\hline & Mange mite & 4 & $0(0-10)$ & $<0.01$ \\
\hline & Wart & 3 & $0(0-7.5)$ & $<0.01$ \\
\hline & "Liver disease" & 3 & $0(0-10)$ & 0.12 \\
\hline & “Milk contaminant” & 2 & $0(0-6.7)$ & $<0.05$ \\
\hline \multirow{9}{*}{$\begin{array}{l}\text { Possibility to prevent } \\
\text { locally }\end{array}$} & Rabies & 20 & $3.3(2-8)$ & Ref \\
\hline & Anthrax & 19 & $10(0-10)$ & $<0.05$ \\
\hline & Brucellosis & 6 & $0(0-10)$ & $<0.05$ \\
\hline & TB & 9 & $0(0-7.5)$ & $<0.05$ \\
\hline & Teania & 8 & $0(0-10)$ & 0.08 \\
\hline & Mange mite & 4 & $0(0-10)$ & $<0.01$ \\
\hline & Wart & 3 & $0(0-7.5)$ & $<0.01$ \\
\hline & "Liver disease" & 3 & $0(0-8.3)$ & $<0.05$ \\
\hline & “Milk contaminant” & 2 & $0(0-6.7)$ & $<0.01$ \\
\hline \multirow{9}{*}{ Mortality rate in cattle } & Rabies & 20 & $10(6.7-10)$ & $\mathrm{Na}$ \\
\hline & Anthrax & 19 & $8(0-10)$ & $<0.01$ \\
\hline & Brucellosis & 6 & $0(0-6)$ & $<0.01$ \\
\hline & TB & 9 & $0(0-5)$ & $<0.01$ \\
\hline & Teania & 8 & $0(0-5)$ & $<0.01$ \\
\hline & Mange mite & 4 & $0(0-10)$ & $<0.01$ \\
\hline & Wart & 3 & $0(0-6)$ & $<0.01$ \\
\hline & "Liver disease" & 3 & $0(0-6.7)$ & $<0.01$ \\
\hline & "Milk contaminant" & 2 & $0(0-6)$ & $<0.01$ \\
\hline \multirow[t]{9}{*}{ Morbidity rate in cattle } & Rabies & 20 & $6.7(2-10)$ & $\mathrm{Na}$ \\
\hline & Anthrax & 19 & $3.3(0-7.5)$ & 0.19 \\
\hline & Brucellosis & 6 & $0(0-10)$ & $<0.01$ \\
\hline & TB & 9 & $0(0-10)$ & 0.12 \\
\hline & Teania & 8 & $0(0-10)$ & 0.12 \\
\hline & Mange mite & 4 & $0(0-10)$ & 0.16 \\
\hline & Wart & 3 & $0(0-10)$ & $<0.01$ \\
\hline & "Liver disease" & 3 & $0(0-10)$ & 0.14 \\
\hline & "Milk contaminant" & 2 & $0(0-8.3)$ & $<0.05$ \\
\hline
\end{tabular}

* If a group did not identify a disease, a score of zero was assigned

**Kruskal Wallis pairwise comparison test to test for differences in the ranking of rabies and the ranking of other diseases 


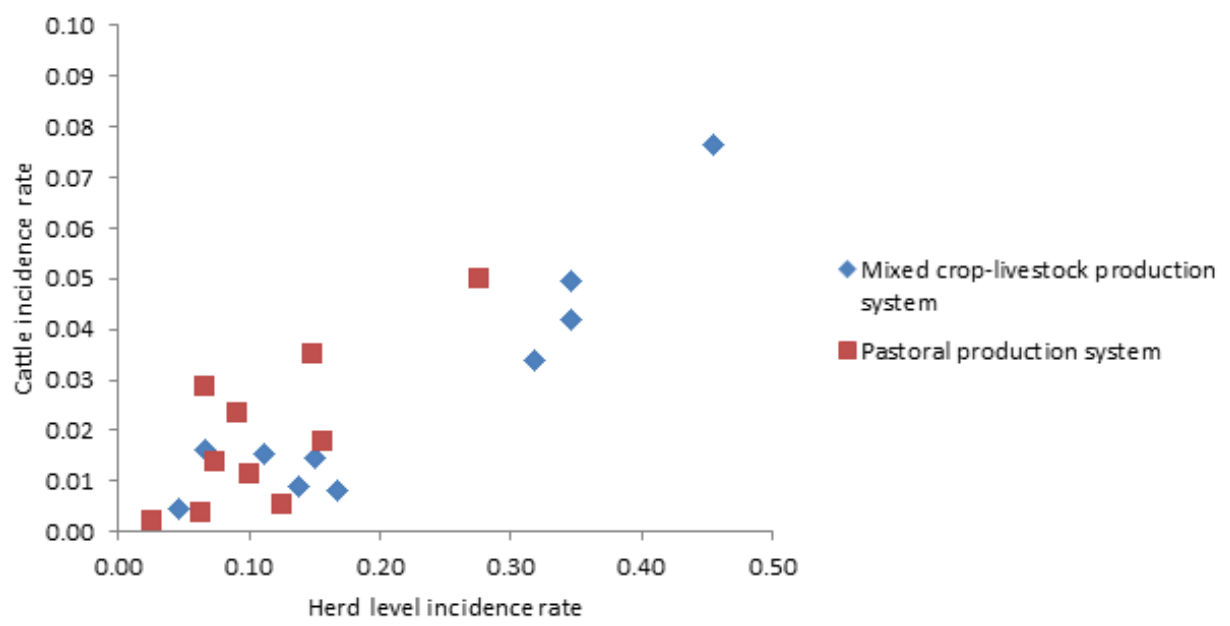

Figure 4.3 Distribution plot of village-specific rabies incidence rates at herd level and cattle level per cattle production system.

Table 4.3: Average herd-level and cattle-level incidence rates of rabies per village within each district and farming system.

\begin{tabular}{clcccc}
\hline Farming system & District & $\begin{array}{c}\text { Number of } \\
\text { herds }\end{array}$ & $\begin{array}{c}\text { Herd-level } \\
\text { incidence (\%) }\end{array}$ & $\begin{array}{c}\text { Number of } \\
\text { cattle }\end{array}$ & $\begin{array}{c}\text { Cattle-level } \\
\text { incidence (\%) }\end{array}$ \\
\hline & Munessa & 119 & 22.7 & 1345 & 2.1 \\
& Lemuna-Bilbilo & 129 & 19.4 & 1871 & 2.4 \\
Mixed crop-livestock & Average & & $\mathbf{2 0 . 9}$ & & $\mathbf{2 . 2}$ \\
\hline & Yabelo & 134 & 6.7 & 2171 & 1.1 \\
& Dugda Dawa & 150 & 14.7 & 2325 & 2.2 \\
Pastoral & Average & & $\mathbf{1 0 . 9}$ & & $\mathbf{1 . 7}$ \\
\hline & Overall average & & $\mathbf{1 5 . 6}$ & & $\mathbf{1 . 9}$ \\
\hline
\end{tabular}

\subsubsection{Economic losses related to rabies}

The average direct economic losses resulting from rabies mortality in cattle during the period September 2013 to August 2014 were approximately 49 USD per herd in the mixed croplivestock system and 51 USD in the pastoral system. There were no significant differences in the economic losses due to mortality per herd between the districts within a system. However, the economic losses per affected herd were significantly higher $(\mathrm{p}<0.01)$ in the pastoral production system (Table 4.4). Average losses per affected herd equalled 228 USD per herd in the mixed crop-livestock system and 478 USD in the pastoral system, although losses per affected herd were rather negatively skewed (Table 4.4). The number of cattle affected per 
affected herd was on average higher in the pastoral districts but not significantly different $(\mathrm{p}>$ 0.05) from the corresponding number in the mixed crop-livestock districts.

Table 4.4 Number of cattle affected by rabies per herd and resulting economic losses for the two cattle farming systems during the period September 2013-August 2014 presented by median and range

\begin{tabular}{|c|c|c|c|}
\hline \multirow[b]{2}{*}{ Level of loss } & \multicolumn{2}{|c|}{ Average loss per production system } & \multirow[b]{2}{*}{ p-value } \\
\hline & $\begin{array}{c}\text { Mixed crop- } \\
\text { livestock }\end{array}$ & Pastoral & \\
\hline Number of cattle affected per affected herd & $1(1-4)$ & $2(1-5)$ & $>0.05$ \\
\hline Losses per affected herd (USD*) & $147(48-1016)$ & $429(173-1140)$ & $<0.01$ \\
\hline
\end{tabular}

\subsection{Discussion}

This study provides an estimate of the direct economic losses due to rabies in two cattle farming systems in Ethiopia. To the best knowledge of the authors, this is the first quantitative assessment of economic losses due to cattle rabies reported for an African region.

In this study, the disease incidence estimated in this study relies on the farmers' ability to recognise and identify rabies, as we considered this the best option available in the situation, where registered data on rabies incidence in cattle is unavailable. The participatory method we used to verify whether-livestock rabies was present in the study area has been validated and used in several studies in livestock-keeping communities (e.g.Rufael et al. 2008). Livestock owners' ability to effectively identify livestock diseases, especially in areas with poor diagnostic facilities for livestock diseases, is also documented in literature (Jost et al.,2007;Rufael et al., 2008). This is supported by laboratory records from the Ethiopian Public Health Institute over 2010-2014 (unpublished data); suspected cattle rabies cases were confirmed positive, in more than $80 \%$ of the submitted samples of which the majority was submitted by farmers. Previous reports from the same institute similarly showed that between $62.5 \%$ and $100 \%$ of the annually reported suspected rabies cases in cattle were confirmed positive during a ten-year period (1990-2000) (Yimer et al., 2002). Comparable results were obtained in a study in Tanzania, where the reported recognition rate of livestock owners for rabies was above 74\% (Lembo et al., 2008). Although we have not sampled suspected cattle cases to confirm the livestock owners' ability to effectively identify rabies, we expect that the performed retrospective diagnosis of rabies based on clinical signs did not compromise the reliability of the estimates due to the obvious symptoms and invariably fatal consequence of rabies. 
To minimise response bias, neither the livestock owners nor the local facilitators were informed during the group discussion that the main disease of interest was rabies. Moreover, during the household surveys, neighbours were approached to verify survey responses indicating the presence of affected and or suspected cattle in the surveyed household. As such there were no indications of information bias, although this would certainly have resulted in higher incidence rates.

Surveyed households were not selected at random from the entire Ethiopian population of livestock- owning households but from a predefined number of villages (e.g. 20) from 4 districts in 2 zones, where districts were selected for their large cattle population sizes. As such this selection procedure could have resulted in some selection bias with respect to the overall representativeness of the indicated incidence rates. Uncertain are, however, the implications of this source of bias due to a lack of knowledge on dog population size as well as on the rabies dog incidence rate among districts.

Rabies was the only zoonotic disease identified by all discussion groups in both farming systems. Independent of the farming system, it was ranked highest in terms of perceived risk to human health and mortality rate in cattle, but not for its possibility to prevent or treat. Despite the availability of safe and effective animal and human vaccines, livestock farming communities in Ethiopia appear not to practise rabies prevention and control methods in dogs. This could be the result of a low level of awareness about preventive options and poor vaccine availability (Jemberu et al., 2013; Kabeta et al., 2015).

In total 532 livestock-owning households were surveyed with 248 in the mixed crop-livestock system and 284 in the pastoral system. Although the herd sample sizes per system resulted from the pragmatic selection of 10 villages per system, the surveyed numbers were in line with the required sample size to estimate the proportion of cattle owners experiencing rabies in their cattle during the last year based on the assumption of $20 \%$ rabies affected herds at a $95 \%$ confidence level and a 5\% desired absolute precision, which would require a sample size of 246 households per system (Thrustfield, 2005).

The incidence rates of cattle rabies estimated in this study are higher than those reported by Jemberu et al., (2013) and Knobel et al., (2005). The study of Jemberu et al., (2013) resulted in an estimated incidence of 18.9 per 100,000 head of cattle. This study analysed the situation in an urban area in northern Ethiopia, where cattle are kept partly indoors and have, therefore, reduced contacts with dogs. Moreover, the study was based on data of officially reported cases, resulting in a conservative estimation. The study by Knobel et al., (2005) provided a continentwide incidence estimation, derived from cattle samples submitted to central veterinary laboratories, which revealed an annual rabies incidence of $0.5-2$ deaths per 100,000 head of 
cattle. By considering an underreporting rate of 10, the final incidence was estimated at $5-20$ deaths per 100,000 head of cattle. Although in current study estimations might to some extent be specific for the selected study region, the large differences in comparison to the reported values of Jemberu et al., (2013) and Knobel et al., (2005) indicate once more the incompleteness of the veterinary records and their unsuitability for impact assessment studies (Tefera et al. , 2002; Adane et al., 2013; EHNRI, 2012).

The economic losses due to rabies estimated in this study assumed the opportunity cost of losing cattle from rabies to be equal to the market value of cattle. Mortality, in itself, does not seem to influence the market situation, as most of the cattle raised in the studied production systems are for subsistence purposes. However, mortality can influence food security in affected households. Livestock owners are aware that rabies is a fatal disease once clinical signs have manifested. Therefore, livestock owners kill cattle that are considered to be rabid and slaughter cattle that have been bitten by a rabid dog. This occurs in both of the zones in this study. The economic losses per herd are reduced slightly, as communities share and consume boiled meat from cattle bitten by a suspected rabid dog, by trimming off the bitten part.

Jemberu et al. (2013) found that only 50\% of the exposed cattle actually developed rabies and died. From a risk-neutral perspective, the average economic burden per herd could be reduced by keeping the exposed cattle for the incubation period, as only a proportion of the exposed cattle actually develop rabies. If the probability of developing rabies is greater than the ratio between the loss due to mortality and the market value of the cattle, i.e., ((market price-salvage value) / market price), then it would be economical to slaughter the exposed cattle. Based on the data collected in this study, the breakeven probabilities are 0.67 ((248 USD market price 81 USD salvage value) / 248 USD market price) for the mixed crop-livestock system and 0.72 ((280 USD market price - 77 USD salvage value) / (280 USD market price)) for the pastoral system. This would mean that, on average, it is economical to slaughter cattle as a precautionary measure if the chance that cattle will develop rabies after exposure exceeds $67 \%$ in the mixed crop-livestock system and $72 \%$ in the pastoral system, given the indicated market prices and salvage values.

The indirect impacts of an extended loss of milk production and draft power were not considered in this study, as the replacement process in cattle herds in the studied farming systems follows the natural process. Immediate replacement by purchasing cattle is restricted by a lack of liquidity in these livestock systems. Thus our analysis may have underestimated the magnitude of the economic losses. On the other hand, the average losses per herd might also have been overestimated, as we did not account for the saved costs that are no longer spent to maintain the animal. We expect these saved costs to be negligible as both of the cattle production systems studied are low-input production systems. There are negligible costs saved 
by farmers when a cattle is lost from a herd, as they use communal grazing land and watering points and temporary cattle housing in their backyards (Tegegne et al., 2009). In this study, we did not consider cattle raised in the market-oriented commercial production system $(<1 \%$ of cattle population), as they are kept in an intensive system with minimal direct contact with dogs, which are the main source of rabies for livestock.

We found higher number of cattle rabies incidences in affected herds in the pastoral system compared to the mixed crop-livestock system. Consequently, the estimated economic losses per affected herd are also higher in the pastoral system indicating higher expected economic losses in affected households as well. This could be due to the fact that pastoral herds are larger in size and more animals are bitten once a rabid dog enters a herd. Assuming an average annual expenditure of rural households of 1014 USD as a proxy for income (CSA, 2012), the direct economic losses due to rabies per affected pastoral herd is equivalent to about $5 \%$ of the average income. This can be seen as a significant burden, given that a loss of more than $10 \%$ of income is seen as a benchmark for a catastrophic shock (Shankar et al., 2012). When accounting for the indirect economic losses, this percentage is even higher and the consequences are thus more severe for pastoral households, because of their total dependency on livestock for their livelihood and food security. Although rabies has a lower direct economic impact at household level in comparison to other cattle diseases, such as FMD (Jemberu et al., 2014), our results suggest that rabies still presents a significant economic burden, particularly for pastoral households.

The estimated incidence rates found in this study can be extrapolated to provide a national estimate for the economic losses caused by rabies in cattle. Assuming that $83 \%$ and $17 \%$ of the 56.7 million cattle currently held in Ethiopia are raised in mixed crop-livestock and pastoral livestock production systems, respectively (Ofcansky and Berry, 1991; CSA, 2012), and using the average cattle herd sizes (13.0 cattle per mixed crop-livestock herd and 15.5 cattle per pastoral herd) as well as the average direct economic losses resulting from rabies mortality per system (49 USD per herd in mixed crop-livestock systems and 51 USD per herd in pastoral systems), then the annual national economic loss due to rabies in cattle is estimated to be about 209 million USD. This estimate falls within the range estimated by Hampson et al., (2015) in a study on the global burden of canine rabies, of between 10 and 412 million USD per year for comparable countries. Extrapolation to the national level requires numerous assumptions, but the estimate does provide some indication of the extent of national losses, the size of which lends support for strengthening initiatives towards controlling the disease (Onono et al., 2014).

Studies show that it is uneconomical to vaccinate cattle against rabies, primarily because the risk of individual animals contracting rabies is low (Yakobson et al., 2015). Given the size of the economic losses in cattle and considering that domestic dogs are the major sources of the 
disease for cattle, intervention through mass dog vaccination seems to be cost effective from a broader perspective, as the benefits would be shared by the public health sector. This has been demonstrated in many developing countries, such as for rabies in Chad and brucellosis in Mongolia (Zinsstag et al., 2007; Zinsstag et al., 2009). Assuming that a farmer household in rural Ethiopia has an average of 3-5 dogs and the vaccination costs per dog are 5-10 USD annually, and given the average losses found in this study of 49 USD per herd per year in mixed crop-livestock zones and 52 USD in pastoral zones, the saved economic losses would be similar or higher than the cost of vaccinating dogs. This suggests that dog vaccination is cost effective, even when the benefits for public health are not considered. However, livestock owners may perceive the cost-effectiveness differently as the losses due to rabies are mainly in kind and do not result in actual reductions of revenues, while the costs for dog vaccination result in actual expenditures which might go beyond the financial resources of most livestock owners.

\subsection{Conclusion}

This study explored the incidence of rabies in cattle herds and related economic losses. Results indicated a large impact on the livelihoods of affected households in the pastoral system, as they completely dependent on livestock for their livelihoods. Our economic analysis shows that the saved economic losses from mass dog vaccination are similar or higher than the costs of vaccination, even without considering the benefits for public health. This provides support for rabies control at the dog population level.

\section{Acknowledgements}

We would like to thank the village elders who contributed to the group discussions, the farmers who participated in the questionnaire survey and the animal health personnel in the study districts for their assistance during data collection. 


\subsection{References}

Adane, K., Muluye, D., Abebe, M., 2013. Processing medical data: a systematic review. Archives of Public Health, 71(1), 27.

Alemayehu, K., 2011. Value Chain assessment of beef cattle production and marketing in Ethiopia. Challenges and Opportunities of linking smallholder farmers to the markets. LRRD, 23(12), 255-265.

Ali, A., Yimer E., Mengistu F., Hussen, K., Tafese, K ., 2010. Overview of Rabies in and around Addis Ababa, in Animals Examined in EHNRI Zoonoses Laboratory Between, 2003 and 2009. Ethiop. Vet. J, 14 (2), 91-101.

Catley, A., Irungu, P., Simiyu, K., Dadye, J., Mwakio, W., Kiragu, J., Nyamwaro, S. O., 2002. Participatory investigations of bovine trypanosomiasis in Tana River District, Kenya. Med and Vet Ent, 16(1), 55-66.

CSA (Central Statistical Agency), 2012. Household Consumption and Expenditure (HCE) Survey 2010/11. The Federal Democratic Republic of Ethiopia Central Statistical Agency. Analytical report. Addis Ababa, Ethiopia. pp. 47-69.

Deressa A, Ali A, Beyene M, Newaye Selassie B, Yimer E, et al., 2010. The status of rabies in Ethiopia: A retrospective record review. Ethiopian Journal of Health Development 24:127-132.

EHNRI (Ethiopian Health and Nutrition Research Institute). 2012. Proceedings of The National Workshop on Rabies Prevention and Control in Ethiopia. The Ethiopian Health and Nutrition Research Institute. October 18-19, 2012. Adama, Ethiopia.

Fafchamps, M., Kebede, B., Quisumbing, A. R., 2009. Intra-household Welfare in Rural Ethiopia. Oxford Bulletin of Economics and Statistics, 71(4), 567-599. doi: DOI 10.1111/j.1468-0084.2009.00553.x.

Hampson, K., Coudeville, L., Lembo, T., ... Global Alliance for Rabies Control Partners for Rabies Prevention., 2015. Estimating the global burden of endemic canine rabies. PLoS Negl. Trop. Dis, 9(4), e0003709. doi: 10.1371/journal.pntd.0003709.

Jemberu, W. T., Molla, W., Almaw, G., Alemu, S., 2013. Incidence of rabies in humans and domestic animals and people's awareness in North Gondar Zone, Ethiopia. PLoS Negl. Trop. Dis, 7(5), e2216. doi: 10.1371/journal.pntd.0002216

Jemberu, W. T., Mourits, M. C., Woldehanna, T., Hogeveen, H., 2014. Economic impact of foot and mouth disease outbreaks on smallholder farmers in Ethiopia. Prev. Vet. Med, 116(1-2), 26-36. doi: 10.1016/j.prevetmed.2014.06.004.

Jibat, T., Hogeveen, H., Mourits, M. C., 2015. Review on dog rabies vaccination coverage in Africa: a question of dog accessibility or cost recovery? PLoS Negl. Trop. Dis, 9(2), e0003447. doi: 10.1371/journal.pntd.0003447.

Jost, C. C., Mariner, J. C., Roeder, P. L., Sawitri, E., Macgregor-Skinner, G. J., 2007. Participatory epidemiology in disease surveillance and research. OIE Revue Scientifique et Technique, 26(3), 537-549. 
Kabeta, T., Deresa, B., Tigre, W., Ward, M. P., Mor, S. M., 2015. Knowledge, Attitudes and Practices of Animal Bite Victims Attending an Anti-rabies Health Center in Jimma Town, Ethiopia. PLoS Negl. Trop. Dis, 9(6), e0003867.

Kahn, C., Line, S., Co, M., 2010. The Merck Veterinary Manual. A handbook of diagnosis, therapy, and disease prevention and control for the veterinarian. Eds: . Inc., New Jersey, USA, 905-908.

Knobel, D. L.Cleaveland, S.Coleman, P. G.Fevre, E. M.Meltzer, M. I.Miranda, M. E.Shaw, A.Zinsstag, J.Meslin, F. X., 2005. Re-evaluating the burden of rabies in Africa and Asia. Bul. Wor. Health Org, 83(5), 360-368. doi: /S0042-96862005000500012.

Lembo, T., Hampson, K., Haydon, D. T., Craft, M., Dobson, A., Dushoff, J., Ernest, E., Hoare, R., Kaare, M., Mlengeya, T., Mentzel, C., Cleaveland, S., 2008. Exploring reservoir dynamics: a case study of rabies in the Serengeti ecosystem. J. Appl. Ecol., 45(4), 12461257. doi: 10.1111/j.1365-2664.2008.01468.x.

MOARD, 2007. Livestock Development Master Plan Study. Phase I Report -Data collection and analysis. Volume V - Policy and Institutions. Ministry of Agriculture and Rural Development, Government of Ethiopia, Addis Ababa, Ethiopia.

Nicholson, K. G., 1990. Rabies. The Lancet, 335(8699), 1201-1202. doi: http://dx.doi.org/10.1016/0140-6736(90)92710-Y. [Originally published as Volume 1, Issue 8699].

Ofcansky, T. P., Berry, L., 1991. Ethiopia: A country study. Washington: GPO for the Library of Congress.

Okell, C. N., Pinchbeck, G. P., Stringer, A. P., Tefera, G., Christley, R. M., 2013. A communitybased participatory study investigating the epidemiology and effects of rabies to livestock owners in rural Ethiopia. Prev. Vet. Med., 108(1), 1-9. doi: DOI 10.1016/j.prevetmed.2012.07.003.

Onono, J. O., Wieland, B., Rushton, J., 2014. Estimation of impact of contagious bovine pleuropneumonia on pastoralists in Kenya. Prev. Vet. Med., 115(3-4), 122-129. doi: 10.1016/j.prevetmed.2014.03.022.

Randall, D.A.,Williams, S. D.,Kuzmin, I. V., Rupprecht, C. E.,Tallents, L. A.,Tefera, Z.,Argaw, K.,Shiferaw, F.,Knobel, D. L., Sillero-Zubiri, C., 2004. Rabies in endangered Ethiopian wolves. Emerg. Infec. Dis., 10(12), 2214-2217.

Razali, N. M., Wah, Y. B., 2011. Power comparisons of shapiro-wilk, kolmogorov-smirnov, lilliefors and anderson-darling tests. Journal of Statistical Modeling and Analytics, 2(1), 21-33.

Rufael, T., Catley, A., Bogale, A., Sahle, M., Shiferaw, Y., 2008. Foot andmouth disease in the Borana pastoral system in southern Ethiopiaand implications for livelihoods and international trade. Trop. Anim. Health Prod. 40, 29-386. 
Shankar, B., Morzaria, S., Fiorucci, A., Hak, M., 2012. Animal disease and livestock-keeper livelihoods in Southern Cambodia. International Development Planning Review, 34(1), 39-63. doi: Doi 10.3828/Idpr.2012.3.

Shapiro, S. S., Wilk, M. B., 1965. An analysis of variance test for normality. Biometrika, 591611.

Sillero-Zubiri, C., Switzer, D., 2004. Management of wild canids in human-dominated landscapes. Canids: foxes, wolves, jackals and dogs. Status survey and conservation action plan. $2^{\text {nd }}$ Edition. IUCN Canid Specialist Group, Gland, Switzerland and Cambridge, UK, 257-266.

Solomon, A., 2003. Livestock marketing in Ethiopia: a review of structure, performance, and development initiatives: ILRI (ILCA and ILRAD). Report series volume 52.

Tambi, N. E., Maina, W. O., Ndi, C., 2006. An estimation of the economic impact of contagious bovine pleuropneumonia in Africa. Rev. sci. tech. Off. int. Epiz, 25(3), 999-1011.

Tefera, G., Yimer, E., Geyid, A., 2002. Endemic existence of rabies in Ethiopia. Eth. Med. J. 1, 40(2), 163-170.

Tegegne, A., Tesfaye, M.., Tesfaye,D., Worku, T., Eshete, D., 2009. Transhumance cattle production system in North Gondar, Amhara Region, Ethiopia: Is it sustainable? IPMS (Improving Productivity and Market Success) of Ethiopian Farmers Project. Working Paper No. 14. ILRI (International Livestock Research Institute), Nairobi, Kenya. 73 pp.

Tepsumethanon, V., Wilde, H., Meslin, F. X., 2005. Six criteria for rabies diagnosis in living dogs. J Med Assoc Thai, 88(3), 419-422.

Tolera, A., Abebe, A., 2007. Livestock production in pastoral and agro-pastoral production systems of southern Ethiopia. LRRD, 19(12), 4-7.

Thrustfield, M. 2005. Veterinary epidemiology,3rd edition UK, Black well Science Ltd, pp: 182-189.

Wunner, W. H., Conzelmann, K.K., 2013. Rabies virus. AC Jackson. Rabies, $3^{\text {rd }}$ Edition. Oxford, UK: Acad Press/Elsevier, 17-60.

Yakobson, B.,Taylor, N., Dveres, N., Rozenblut, S.,Tov, Boris E.,Markos, M.,Gallon, N., Homer, D.,Maki, J., 2015. Cattle rabies vaccination-A longitudinal study of rabies antibody titres in an Israeli dairy herd. Prev. Vet. Med, 121(1-2), 170-175. doi: http://dx.doi.org/10.1016/j.prevetmed.2015.05.004.

Yimer,E.,Bethelehem, N.,Girma, T.,Yared, M.,Yosef, B.,Badeg, Z.,Mekoro, B.,Abebe, B., 2002. Situation of rabies in Ethiopia: a retrospective study 1990-2000. Eth. J. Health Dev., 16(1), 105-112.

Zinsstag, J., Durr, S., Penny, M. A., Mindekem, R., Roth, F., Gonzalez, S. M., Naissengar, S., Hattendorf, J., 2009. Transmission dynamics and economics of rabies control in dogs and humans in an African city. Proc Natl Acad Sci U S A, 106(35), 14996-15001. doi: DOI 10.1073/pnas.0904740106. 
Zinsstag, J., Schelling, E., Roth, F., Bonfoh, B., de Savigny, D., Tanner, M., 2007. Human benefits of animal interventions for zoonosis control. Emerg. Inf. Dis., 13(4), 527-531.

Zinsstag, J., Schelling, E., Waltner-Toews, D., Tanner, M., 2011. From "one medicine" to "one health" and systemic approaches to health and well-being. Prev. Vet. Med, 101(3-4), 148-156.

\section{Supplementary information 4.1: Set up of household survey}

During the last year, did you experience:

1) Any rabid cases among your cattle

- If you experienced rabid cases, how many cattle were affected?

- What were their sex and age?

- Could you describe the clinical signs as shown by the rabid cattle? and/or 2) any cattle being bitten by a suspected rabid dog?

- If you experienced any cattle being bitten by a suspected rabid dog, how many cattle were bitten?

- What were their sex and age?

- Could you describe the clinical signs shown of the biting rabid animal?

\section{Supplementary information 4.1: Clinical signs of rabies in cattle (source: Kahn et al., 2010)}

Rabid animals of all species usually exhibit typical signs of Central Nervous System disturbance. The most reliable signs, regardless of species, are acute behavioural changes and unexplained progressive paralysis. Behavioural changes include sudden anorexia, signs of apprehension or nervousness, irritability and hyper excitability (including priapism). Ataxia, altered phonation and changes in temperament are apparent. Uncharacteristic aggressiveness may develop - a normally docile animal may suddenly become vicious. Rabid animals commonly lose their fear of people. Cattle with rabies can be dangerous, attacking and pursuing people and other animals. Lactation ceases abruptly in dairy cattle. The usual placid expression is replaced by one of alertness. The eyes and ears follow sounds and movements. A common clinical sign is a characteristic abnormal bellowing, which may continue intermittently until shortly before death. 



\section{Chapter 5}

\section{Estimating the burden of rabies in Ethiopia by dog bite victims tracing}

Tariku Jibat Beyene, Monique C.M. Mourits, Abraham Haile Kidane, Henk Hogeveen Under review by PLOS ONE Journal 


\begin{abstract}
In developing countries where financial resources are limited and numerous interests compete, there is a need for quantitative data on the public health burden and costs of diseases to support intervention prioritization. This study aimed at estimating the health burden and post-exposure treatment (PET) costs of canine rabies in Ethiopia by an investigation of exposed human cases. Data on registered animal bite victims during the period of one year were collected from health centers in three districts, i.e. Bishoftu, Lemuna-bilbilo and Yabelo, to account for variation in urban highland and lowland areas. This data collection was followed by an extensive case search for unregistered victims in the same districts as the registered cases. Victims were visited and questioned on their use of PET, incurred treatment costs and the behavioral manifestations of the animal that had bitten them. Based on the collected data PET costs were evaluated by financial accounting and the health burden was estimated in Disability-Adjusted Life Years (DALYs). In total 655 animal bite cases were traced of which $96.5 \%$ was caused by dog bites. $73.6 \%$ of the biting dogs were expected to be potentially rabid dog. Annual rabid dog exposures were estimated per evaluated urban, rural highland and rural lowland district at, respectively, 135, 101 and 86 bites, which led, respectively, to 1, 4 and 3 deaths per 100,000 inhabitants. In the same district order average costs per completed PET equalled to 23, 31 and 40 USD, which was significantly higher in rural districts. Weighted extrapolation of results to the national level indicated an annual estimate of approximately 3,000 human deaths resulting in about 93,000 DALYs per year and 97,000 exposed persons requiring on average 2 million USD treatment costs per year countrywide. These estimations of the burden of rabies to the Ethiopian society provide decision makers insights into the potential benefits of implementing effective interventions.
\end{abstract}




\subsection{Introduction}

Rabies is a viral infection that infects all mammals. Estimations on the global burden of rabies indicate a health impact of 59,000 human deaths per year, a loss of 3.7 million DALYs per year and about 8.6 billion USD of economic losses mainly due to premature deaths (productivity losses) and post-exposure treatment (PET) costs (Hampson et al., 2015).

Most of the human death cases occur in Asian and African countries (Knobel et al., 2005). Of these countries, Ethiopia is one of the worst affected (Hampson et al., 2015), with domestic dogs as the major transmitters of the disease to humans (Wunner and Jackson, 2010). Dog management is, however, poor and dog rabies vaccination is limited to a limited number of dogs in urban centers (Ali et al., 2010). The large dog population size in combination with poor dog management contributes to a high endemicity of canine rabies in Ethiopia (Yimer et al., 2002).

Basic information on the health and economic burden, e.g., on the amount of lives lost due to rabies and on the costs of treatment amongst those at risk, is crucial in the development of sustainable control programs. In most rabies endemic countries, reliable reports of incidence data on rabies and rabies exposure are lacking. Official reports generally underestimate the true number of human rabies cases and hence the true burden (Hampson et al., 2015; Taylor and Knopf, 2015). For instance, in sub-Sahara African countries like Tanzania, the incidence of human rabies predicted on the basis of active surveillance data on bite incidences was up to 100 times larger than the officially recorded number of deaths (Cleaveland et al., 2002). In Ethiopia, the national annual estimates from official reports indicate 12 exposure cases per 100,000 heads of inhabitants and 1.6 rabies deaths per 100,000 heads of inhabitants (Deressa et al., 2013). However, the actual numbers are expected to be higher as many cases are not reported (Moges, 2015).

The reliability of the reported incidence data is expected to differ among the regions in Ethiopia due to geographical as well as cultural differences. For instance, in rural Ethiopia, individuals who are exposed to rabies often see traditional healers for the diagnosis and treatment of the disease because of cultural background, lack of knowledge or limited accessibility to medical treatment. These widespread traditional practices of handling rabies cases might interfere with medical treatment seeking practice, resulting in an underreporting of the actual number of rabies cases and its related health burden. To fill the disparities between officially recorded and likely occurring rabies cases, researchers have applied approaches like extensive animal bite case searching, and predictive modelling (Ly et al., 2009; Tenzin et al., 2011; Hossain et al., 2012; Hampson et al., 2008). 
Rabies in exposed humans is preventable when an effective post-exposure treatment (PET) is applied immediately after exposure which includes post- exposure prophylaxis (PEP), wound washing, antibiotic and tetanus antitoxin administration. With respect to the applied PEP vaccine WHO recommends to use inactivated rabies vaccines produced in cell culture or embryonated eggs (CCEEV) (WHO, 2007). In Ethiopia, the PEP vaccine used to treat most of the rabies exposures is produced from nervous tissue composed of rabies virus-infected sheep or goat brain inactivated with phenol and also called Nervous Tissue Vaccine (NTV) (WHO, 2017; Piyasirisilp et al., 1999; EHNRI, 2012). This type of vaccine is less immunogenic than the CCEEV and known for its fatality rate and disability rate (Knobel et al., 2005; WHO, 2017). The health burden of rabies in Ethiopia, therefore, not only includes years of life lost due to the premature death following upon infection but also years of life lost due to the premature death following vaccination by NTV and years of life lived with a disability as a consequence of the adverse events of NTV.

At present, although rabies control associated working groups have been established across the regions of Ethiopia, there is no national strategic plan to clearly define targets for rabies control (Coetzer et al., 2016). The relative burden of rabies compared to other neglected tropical diseases is also not known. In countries like Ethiopia, where financial resources are limited, there is a need for quantitative data on the costs of certain diseases to support intervention prioritization (Murray, 1994; Lea, 1993). Moreover, quantification of the health burden enhances the understanding of its long term effects and of the comparative advantages of different levels of treatment and prevention. Therefore, in this study, we aim to estimate the status of rabies in Ethiopia by assessing its health burden and associated PET costs using data obtained by an extensive animal bite case search.

\subsection{Materials and Methods}

A retrospective study was conducted to assess the burden of rabies by collecting data on the incidence of human rabies exposure over the period of one year (September 2013 to August 2014) through an extensive bite case search. Animal bite victims were traced using data collected from the recorded cases at health centers as well as by information obtained from questioning the local community to trace unregistered bite cases. After the tracing, victims (or their family) were contacted and questioned about their use of PET, the health impact of the bite, the incurred costs and on the behavioral manifestation of the biting animal. Based on these data the health burden of rabies was assessed as well as the costs of the applied PET.

\subsubsection{Description of the study population}

This study was conducted in three preselected districts of Ethiopia, i.e. Bishoftu, Lemunabilbilo and Yabelo to account for the geographical variation within the country. Bishoftu is an urban district located at $45 \mathrm{~km}$ South East of Addis Ababa, the capital, with a relatively good 
infrastructure and health centers available within a reasonably short distance. Lemuna-bilbilo is a rural district located in the central highlands of Ethiopia, where most people live on a mixed crop and livestock farming system. Yabelo is a rural district in the lowlands of southern Ethiopia, where the majority of the people are pastoralists keeping livestock for their living. Both rural districts have poor road and health facility coverage. Bishoftu, Lemuna-bilbilo and Yabelo contain, respectively, 140000, 187000 and 101000 inhabitants (CSA, 2013), located in, respectively, 9, 27 and 25 villages. Each district has one or more public health centers delivering medical services including PET and every village within a district has a local health extension worker.

\subsubsection{Data collection}

A list of animal bite victims registered by the districts' health centers in the period between September 2013 and August 2014 was obtained. Animal bite incidences were claimed in 55 out of the total of 61 villages in the three districts. Local health extension workers of these villages were trained in techniques of detailed questionnaire interviews as described by Hampson et al., (2008). After this training, the extension workers contacted and interviewed all dog bite victims using a structured questionnaire. In cases where the victim was deceased or a child, an adult family member was interviewed. The health extension workers also performed an extensive search for non-registered animal bite victims by asking the contacted victims whether they knew other people that were bitten but did not visit a health center. In Ethiopia, most neighborhoods are socially close to each other and are therefore well informed about events happening to households in their village. Something serious as suspected rabid animal bite cases would, therefore, be known within the community (Teshome et al., 2014). The indicated nonregistered bite victims were subsequently contacted and interviewed in the same manner.

\section{Questionnaire survey}

The bite victims were interviewed using a structured questionnaire on: 1) demographic features including age and gender, 2) body part bitten, 3) characteristics of the dog that had bitten them to reflect its behavioural manifestation and ownership (either own, neighbours' or unknown), 4) whether the victim visited a health centre and received PET, 5) whether the person died or survived and 6) the expenditures incurred related to treatment of the bite. The behavioral manifestation and clinical signs of the biting dogs as described by the bite victims were subsequently used to categorize the dog as rabid or non-rabid based on the six-criteria method of Tepsumethanon et al., (Tepsumethanon et al., 2005).

\section{Ethical clearance statement}

The study protocol was approved by the scientific and ethical review committee of the Ethiopian Public Health Institute (Reference No. EPHI-6-13-824). Before administering the questionnaire, the purpose of the study was explained to each victim (or an adult family member 
in case the victim was deceased or a child) as "This study aims at producing evidence on the health and economic burden of rabies in the community. This is done by asking bite victims questions about their demographic situation, the bite injury and followed PET procedure. This is helpful for the policy makers to enforce preventive and control measures. Individual's specific information will neither be published nor passed to any other party”. We also indicated that participation was voluntary and that collected data would be treated as confidential. All respondents approached agreed verbally and participated in the conducted interviews. Permission to conduct interviews was also obtained from the district village health posts and administrative officials in all the study areas.

\subsubsection{Estimating burden of rabies}

The health and economic burden of rabies was estimated based on the cost classification of Jo (Jo, 2014) with the PET costs reflecting the direct costs, consisting of healthcare and nonhealthcare costs expressed in monetary terms, and indirect costs in terms of DALYs as shown in Table 5.1.

Table 5.1 Cost classification for burden of rabies exposure

\begin{tabular}{|c|c|c|}
\hline \multicolumn{2}{|l|}{ Direct costs } & \multirow[t]{2}{*}{ Indirect costs } \\
\hline Health care costs & Non-healthcare costs & \\
\hline Diagnosis & Transportation & Mortality (DALYs) \\
\hline Vaccine & Accommodation and food & Morbidity (DALYs) \\
\hline Wound care and disinfection & Communication & \\
\hline Antibiotics & Productivity loss / & \\
\hline \multirow[t]{2}{*}{ Tetanus immunizations } & opportunity costs of time & \\
\hline & while seeking treatment & \\
\hline
\end{tabular}

\section{Estimating post-exposure treatment costs}

The costs of rabies post-exposure treatment were classified into two direct costs categories: 1 ) healthcare costs reflected by the expenditures for rabies vaccine, antibiotics, tetanus immunizations and disinfection (but not for rabies immunoglobulin as this was not used in the study areas) and 2) non-healthcare costs as the expenses for transport, food, and accommodation, while seeking PET (Table 5.1). Costs were estimated given the interview responses of the bite victims on the number of PEP doses received, the number of visits to the health centres to receive PEP (in case the patient or their parents was not sure or forgot the number of doses we asked the health centres) expenditures during the medical treatment related to transportation, accommodation, food and communication, and the time spent by themselves and their caregivers (if applicable) to account for productivity losses while seeking treatment. 
For patients older than 15 years, the productivity losses while seeking treatment were valued in monetary terms using the GDP per capita daily income. When patients were accompanied during their treatment by an adult caregiver (older than 15 years), related productivity losses were also captured. Considering 568 USD as the Ethiopian average income per capita (World Bank, 2015), we valued the daily average income at 1.6 USD per day. All the costs collected were in Ethiopian birr (ETB) and later converted to US dollars (USD) using the average annual exchange rate for the study period published by the National Bank of Ethiopia (1 USD = 19.22 ETB) (NBE, 2014).

For the estimation of PET costs, we considered PET costs per dog bite irrespective of the rabid status of biting dog and PET costs per sufficient treatment. The average PET costs per dog bite were defined as the sum of health care and non-health care costs including the opportunity cost of time spent while seeking for treatment divided by the total number of bite victims, including those who didn't seek treatment.

A complete PEP dose of NTV consists of 17 doses of vaccine administered consecutively for the first 14 days, with the remaining 3 doses at intervals of 10 days i.e. at day 24, 34 and 44 . A treatment consisting of at least 14 of the 17 PEP doses was considered to be "sufficient", while any treatment consisting of less than 14 doses was regarded to be "insufficient", based on the minimum required number of doses to produce the neutralizing antibody level (WHO, 1984; Ayele et al., 2001). Consequently, the average costs per sufficient treatment only accounted for the costs of patients who successfully received the minimum recommended doses (at least 14 out of the 17 doses) of PEP.

\section{Estimating the health burden of the disease}

We adopted the disability-adjusted life years (DALYs) estimation method developed by the World Health Organization (Prüss-Ustün et al., 2003) to calculate the rabies health burden (Table 5.1). In accordance, DALY is the sum of years of life lost (YLL) due to rabies and adverse effects of NTV and of years lived with disability (YLD) due to adverse effects of NTV.

$$
D A L Y=Y L L+Y L D=\sum_{i=1}^{18}\left(\left(\mathrm{Ndeath}_{i} \times \mathrm{e}_{i}\right)+\left(\text { Ndis }_{i} \times t \times D W\right)\right) \ldots \ldots \ldots \ldots \ldots \ldots \text { Equation } 5.1
$$

YLL is calculated as the number of human deaths (Ndeath) within age category (i) multiplied by the life expectancy $(e)$ of the concerned age category $(i)$, cumulated over all age categories. Eighteen age groups with a 5 years age interval from 0 to 85 years and above were considered ( $i=1 \ldots 18$ ). The average life expectancy for Ethiopia is derived from the Life table estimate of 2013 as published by WHO (WHO, 2013). Similarly, YLD is calculated by multiplying the total number of disability cases (Ndis) of the concerned age group (i), with the duration of the 
disability ( $t$ in years) resulting from the NTV vaccine and its corresponding disability weight (DW) (Knobel et al., 2005), cumulated over the 18 age categories.

More precisely, the DALY estimation is subjected to age weighting and time discounting ( Murray and Lopez, 1996; Murray and Acharya, 1997), following the World Bank recommendation of an age weight of 1 and a time discount rate of 3\% (Mathers et al., 2001). Accordingly, the simplified formulas for YLL and YLD (Equation 5.1) were adjusted.

$$
\begin{aligned}
\mathrm{YLL}=\sum_{i=1}^{18}\left(\frac{\mathrm{KCe}^{\mathrm{ra}}}{(r+\beta)^{2}}\left[e^{-(\mathrm{r}+\beta)\left(\mathrm{L}_{i}+\mathrm{a}\right)}\left[-(\mathrm{r}+\beta)\left(\mathrm{L}_{i}+\mathrm{a}\right)-1\right]-e^{-(\mathrm{r}+\beta) \mathrm{a}}[-(\mathrm{r}+\beta) \mathrm{a}-1]\right]\right. \\
\left.\quad+\frac{1-\mathrm{K}}{r}\left(1-e^{-\mathrm{rl}}\right)\right) \mathrm{N}_{i} \ldots \ldots \ldots \ldots \ldots \ldots \ldots \ldots \ldots \ldots \ldots \ldots \ldots \ldots \ldots \ldots \ldots \ldots \ldots \ldots \ldots \ldots \ldots \ldots \ldots \ldots \ldots \ldots \ldots \ldots \ldots \ldots \\
\end{aligned}
$$

Similarly, by replacing the standard life expectancy in the YLL formula by the duration of disability and accounting for the disability weight, the YLD formula becomes the following

$$
\begin{aligned}
& \mathrm{YLD}=\sum_{i=1}^{18} D W( \frac{\mathrm{KCe}^{\mathrm{ra}}}{(r+\beta)^{2}}\left[e^{-(\mathrm{r}+\beta)\left(\mathrm{L}_{i}+\mathrm{a}\right)}\left[-(\mathrm{r}+\beta)\left(\mathrm{L}_{i}+\mathrm{a}\right)-1\right]-e^{-(\mathrm{r}+\beta) \mathrm{a}}[-(\mathrm{r}+\beta) \mathrm{a}-1]\right] \\
&\left.+\frac{1-\mathrm{K}}{r}\left(1-e^{-\mathrm{rl}}\right)\right) \mathrm{N}_{i} \ldots \ldots \ldots \ldots \ldots \ldots \ldots \ldots \ldots \ldots \ldots \ldots \ldots \ldots \ldots \ldots \ldots \ldots \ldots \ldots \ldots \ldots \ldots \ldots \ldots \ldots \ldots \ldots \ldots \ldots \ldots \ldots \\
& \ldots
\end{aligned}
$$

Where:

$\mathrm{a}=$ average age within age group $\mathrm{i}$ (years)

$r=$ discount rate $(3 \%)$

$\beta=$ age weighting constant $(0.04)$

$\mathrm{K}$ = age-weighting modulation constant (1)

$\mathrm{C}=$ adjustment constant for age-weights (0.1658)

$\mathrm{L}_{\mathrm{i}}=$ life expectancy at age of death in case of YLL or duration of disability in case of YLD (years)

$\mathrm{N}_{\mathrm{i}}=$ number of deaths per age group in case of YLL or number of disabilities per age group in case of YLD

$\mathrm{i}$ = age group (1 to 18$)$

The YLL due to the disease were estimated from the number of deaths captured from the field survey. The disability (YLD) due to the disease itself was considered insignificant as the disease is very acute, leading to death once it develops (Hotez et al., 2014). The field survey primarily indicated the number of death due to rabies disease as specifically asked for. It was, however, not possible to derive a direct DALY estimation due to the adverse effect of NTV because the time lag between NTV administration and occurrence of adverse effects could have been longer 
than the duration of the field survey and because of the difficulty to attribute health problems specifically to the NTV application. As a consequence, the health burden due to NTV was indirectly derived from the number of persons receiving PEP corrected for the number of deaths due to rabies and estimated likelihoods of adverse effects based on the study of Knobel et al (Knobel et al., 2005). YLL was derived from the number of patients who got at least one dose of NTV PEP, multiplied by the expected rate of neurological complications ( 0.4 out of 100 patients receiving NTV) and case fatality rate (17 out of 100 cases of neurological complications) (Knobel et al., 2005). The YLD due to NTV was calculated by multiplying the number of patients who got at least one dose of NTV PEP by the expected rate of neurological complications ( 0.4 out of 100 patients receiving NTV) and corresponding disability weight $(\mathrm{DW}=0.613)$ for a most likely duration of disability of 8 days $(\mathrm{t}=0.022$ years) (Knobel et al., 2005). The weighted and discounted number of deaths and disabilities were computed using the WHO programmed spreadsheet template for DALYs estimation (WHO, 2015).

\subsubsection{Statistical analyses}

Descriptive statistics were used to summarize proportions of rabid suspected dog bite cases per age category, health center visits and sufficient doses of PEP receiving rates. Chi-square test and analysis of variance (ANOVA) were used to compare statistical difference between categorical variables and between group means. Tukey post hoc multiple testing for significance was used for additional exploration of the differences among means in cases of three or more group if means are significantly different from each other. The statistical analysis was done in R statistical software (R 3.3.2) (R Core Team, 2000).

\subsection{Results}

\subsubsection{Rabies exposure}

A total of 655 bite victims was traced and interviewed with 210 cases in Bishoftu (urban), 262 cases in Lemuna-bilbilo (rural highland) and 183 cases in Yabelo (rural lowland) districts. From this total, 23 cases originated from bites by animals other than dogs, i.e. cat (2), cattle (7) donkey (7), horse (1), wild animal/rodents (3), and human (3). These cases were excluded from further analysis, focusing on the remaining $96.5 \%$ cases originating from dog bites.

From the total of, respectively, 208, 250 and 174 dog bite cases in Bishoftu, Lemuna-bilbilo, and Yabelo, 95.7\%, 61.6\% and 56.3\% were reported to health centers. The remainder did not seek medical attention and was identified through contact trace search. Based on the sixcriterion evaluation of a biting dog (Tepsumethanon et al., 2005), 189, 189 and 87 cases in the respective districts were found to be potentially caused by a rabid dog, indicating that on average $73.6 \%$ of the biting dogs was expected to be rabid. 
Figure 5.1 schematically presents the number of dog bite victims who visited health center (450) and who didn't visit health center (182) as identified through the 'snow balling', exercise during the contact tracing. All (632) bite victims were interviewed using a structured questionnaire out of which, according to our six criteria, 360 victims from those who visited health centers and 105 from those who didn't be bitten by a potentially suspected rabid dog. Victims who visited health centers received either sufficient doses of PEP or at least one dose or no PEP at all. Similarly, victims who did not visit a health center either went to traditional healers or nowhere. All the deaths (12) resulted from potentially suspected rabid dog bites. The table below the schematic diagram presents the types of costs considered under each action taken (Direct health cost related to modern PET (post-exposure treatment) and non-PET (traditional/spiritual healers), Direct non-health care costs, and indirect costs in terms of DALY loss (Years of Life Lost (YLL) due to rabies and or adverse effect of the vaccine) and Years Lived with Disability (YLD) due to adverse effect of the vaccine).

When considering only rabid suspected dog bite cases, our data suggest that per 100,000 inhabitants, annually 135, 101 and 86 were exposed to potentially rabid dog cases in Bishoftu, Lemuna-bilbilo and Yabelo, respectively. Using chi-square test, we found a significant difference $(p<0.01)$ in the incidence rate between the urban and rural districts, while no significant difference was found between the highland (Lemuna-bilbilo) and lowland (Yabelo) rural districts $(\mathrm{p}>0.05)$. 


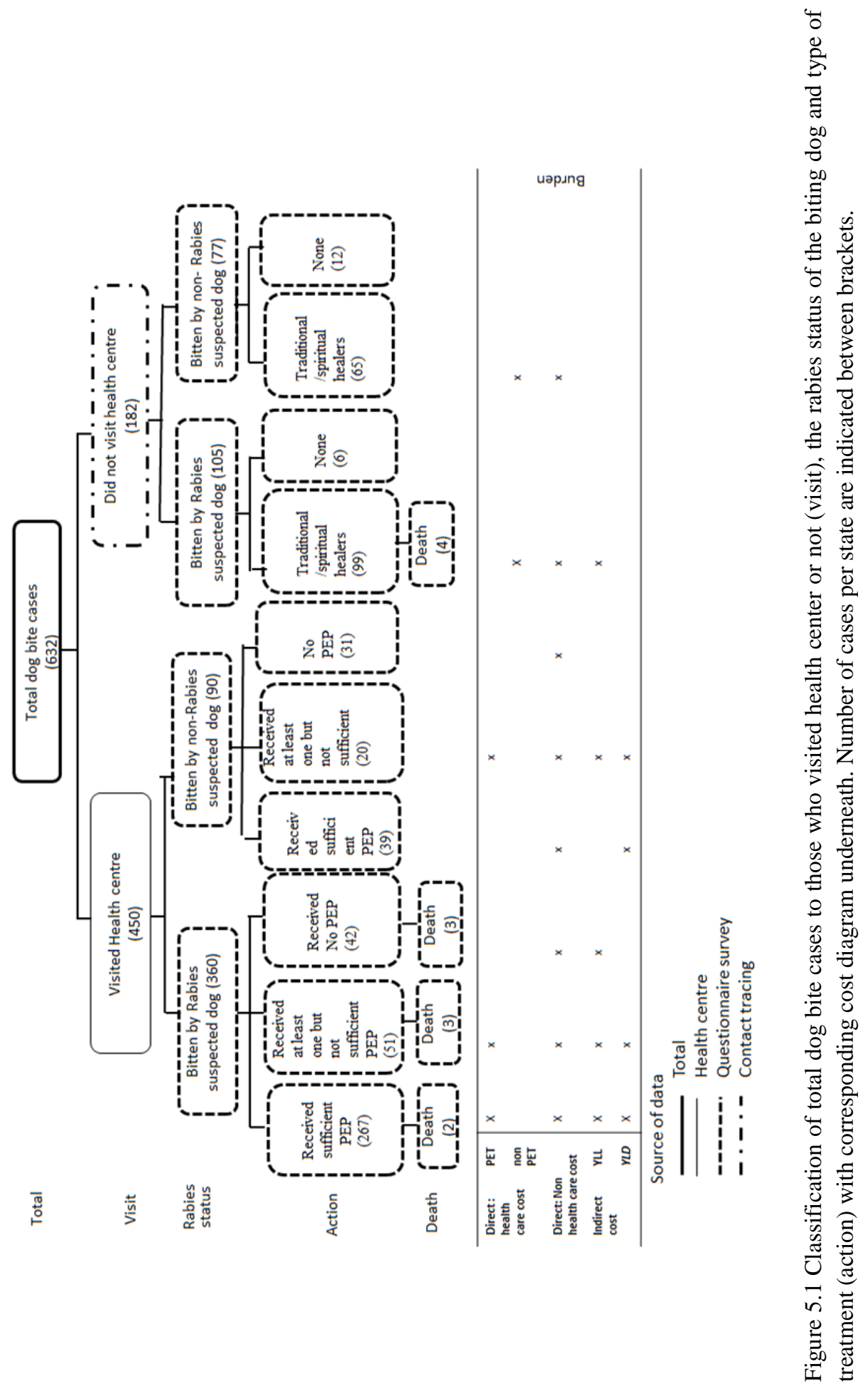


The overall rabid suspected dog bite exposure ratio of male to female was 0.56:0.44. There were no statistical differences between the gender exposure rates in the urban area (Bishoftu), but significantly higher $(\mathrm{p}<0.01)$ exposure rates were observed in males than in females in both rural districts $(\mathrm{p}<0.01)$. The urban district (Bishoftu) have the highest rate of bite victims visiting a health center and receiving sufficient PEP compared to the rural districts (Lemunabilbilo and Yabelo). The proportion of suspected rabid dog bite victims visiting health center was $97 \%$ in Bishoftu, 64\% in Lemuna-bilbilo and 66\% Yabelo, while the proportion of suspected rabid dog bite victims receiving sufficient PEP was 78\% in Bishoftu, 50\% in Lemuna-bilbilo and 29\% Yabelo. Conversely, in the same district order, 2.6\%, 35.5\% and 31\% of the rabid suspected dog bite victims visited traditional/spiritual healers. Rabies-related human death cases compared to the number of total dog bite cases were higher in rural areas than in the urban area. Out of the 12 deaths counted during the survey, one was from Bishoftu, eight from Lemuna-bilbilo and three from Yabelo rural districts (Table 5.2).

Table 5.2 Number of dog bite cases, the number of rabid suspected dog bite cases (RSDB cases), the number of RSDB cases visiting a health center and the number of RSDB cases receiving sufficient PEP (in absolute figures and relative to the number of RSDB cases (italic)) and the number of rabies-related death cases

\begin{tabular}{lllllrrrr}
\hline & $\begin{array}{l}\text { Total } \\
\text { survey }\end{array}$ & Bishoftu & & Lemuna-bilbilo & Yabelo \\
& 632 & -- & 208 & -- & 250 & -- & 174 & -- \\
\hline Dog bite cases & 465 & & 189 & & 189 & & 87 & \\
RSDB cases & 360 & 77.4 & 183 & 96.8 & 120 & 63.5 & 57 & 65.5 \\
RSDB visiting a health centre & & & & & & & \\
RSDB receiving sufficient & 267 & 57.4 & 147 & 77.8 & 95 & 50.3 & 25 & 28.7 \\
PEP & 12 & & 1 & & 8 & & 3 & \\
Rabies deaths & 12 & & & & & & &
\end{tabular}

Age wise, the exposure to rabies suspected dogs in Bishoftu and Lemuna-bilbilo was highest in the 15-29 years age category and in Yabelo in the 5-14 years age category. Very young children and elderly people, i.e. 0-4 years of age and older than 60 years of age, were the least exposed age categories in all the districts. There was no significant difference among age groups across districts regarding the proportion of bite victims that received sufficient treatment $(p>0.05)$. Overall, the age category of 15-29 was the group with the highest proportion of bite victims visiting a health center (85\%) and receiving sufficient PEP (67\%). In terms of visiting a health center and receiving sufficient PEP, the variations between age categories within district were significant in the rural districts ( $\mathrm{p}<0.05$ ) but not in the urban area (Table 5.3). 
Table 5.3 Proportion of RSDB (rabies suspected dog bite) cases per age category

\begin{tabular}{|c|c|c|c|c|}
\hline & Total & $\begin{array}{l}\text { Districts } \\
\text { Bishoftu/ } \\
\text { Urban }\end{array}$ & $\begin{array}{l}\text { Lemuna-bilbilo/ } \\
\text { Rural Highland }\end{array}$ & $\begin{array}{l}\text { Yabelo } \\
\text { Rural-Lowland }\end{array}$ \\
\hline \multicolumn{5}{|c|}{$\begin{array}{l}\text { Proportion of RSDB cases per age } \\
\text { category }\end{array}$} \\
\hline $0-4$ & 3.7 & 3.7 & 4.8 & 1.1 \\
\hline $5-14$ & 30.5 & 27.5 & 29.1 & 40.2 \\
\hline $15-29$ & 38.5 & 44.4 & 35.4 & 32.2 \\
\hline $30-44$ & 16.8 & 14.3 & 19.0 & 17.2 \\
\hline $45-59$ & 6.9 & 6.3 & 7.4 & 6.9 \\
\hline$>60+$ & 3.7 & 3.7 & 4.2 & 2.3 \\
\hline \multicolumn{5}{|c|}{$\begin{array}{l}\text { Proportion of RSDB cases within an } \\
\text { age category visiting a health center }\end{array}$} \\
\hline $0-4$ & 76.5 & 100 & 55.6 & 100 \\
\hline $5-14$ & 74.6 & 98.1 & 61.8 & 58.3 \\
\hline $15-29$ & 84.9 & 96.4 & 77.6 & 70.4 \\
\hline $30-44$ & 62.8 & 96.3 & 38.9 & 60.0 \\
\hline $45-59$ & 81.3 & 91.7 & 71.4 & 83.3 \\
\hline$>60+$ & 82.4 & 100 & 75.0 & 100 \\
\hline \multicolumn{5}{|c|}{$\begin{array}{l}\text { Proportion of RSDB cases within an } \\
\text { age category receiving sufficient PEP }\end{array}$} \\
\hline $0-4$ & 64.7 & 71.4 & 55.6 & 100 \\
\hline 5-14 & 52.8 & 80.8 & 45.5 & 22.8 \\
\hline $15-29$ & 66.5 & 79.8 & 61.2 & 39.3 \\
\hline $30-44$ & 44.9 & 77.8 & 33.3 & 13.3 \\
\hline $45-59$ & 59.4 & 58.3 & 64.3 & 50.0 \\
\hline$>60+$ & 47.1 & 71.4 & 37.5 & 0.0 \\
\hline
\end{tabular}

An overview of bite cases and deaths by bite site and age of patient is given in Table 5.4. Out of the 465 rabies suspected dog bite cases, $6 \%$ occurred in the head/neck region, $19 \%$ on arms/hands, $70 \%$ on the legs and $6 \%$ on the trunk. Overall, proportions of RSDB cases were significantly different across body parts bitten with the highest proportion of bites on the legs and lowest proportion on the trunk. Based on age category, these proportions demonstrated a similar pattern except that for the age category beyond 15-29 years with the lowest proportions of bites on the head/neck part of the body. Of the 12 recorded deaths, $75 \%$ occurred in victims being bitten on the legs. 
Table 5.4 Number of rabid suspected dog bite cases (RSDB cases) and deaths (indicated in brackets) according to bite site and age of the bite victim.

\begin{tabular}{lccccc}
\hline & Head/neck & Arms/hands & Legs & Trunk & Total \\
\hline $0-4$ & 3 & 4 & 9 & 1 & $\mathbf{1 7}$ \\
$5-14$ & 17 & 23 & $94(3)$ & 9 & $\mathbf{1 4 3}$ \\
$15-29$ & $2(1)$ & $36(1)$ & $132(3)$ & 8 & $\mathbf{1 7 8}$ \\
$30-44$ & 2 & 12 & $59(3)$ & $5(1)$ & $\mathbf{7 8}$ \\
$45-59$ & 1 & 6 & 21 & 4 & $\mathbf{3 2}$ \\
$60+$ & 1 & 7 & 9 & - & $\mathbf{1 7}$ \\
\hline Total & $\mathbf{2 6 ( 1 )}$ & $\mathbf{8 8 ( 1 )}$ & $\mathbf{3 2 4}(\mathbf{9})$ & $\mathbf{2 7}(\mathbf{1})$ & $\mathbf{4 6 5}(\mathbf{1 2})$ \\
\hline
\end{tabular}

\subsubsection{Rabies burden}

i. $\quad$ Post-exposure treatment (PET) costs

The average PET costs per bite case were estimated at 21.3, 19.1 and 22.6 USD in Bishoftu, Lemuna-bilbilo and Yabelo, respectively. These estimates included health care and non-health care costs of all 632 dog bite cases which account for all type of treatment received (modern, traditional or none) and did not significantly differ across the three districts $(p=0.12)$. The average total PET costs for those victims who received sufficient PEP doses of NTV were 23.4, 31.5 and 40.1 USD in the same district order. For these average costs per sufficient treatment, significant statistical differences were shown among the three districts.

Table 5.5 indicates the distribution of health care costs and non-health care costs per dog bite case and per sufficient treatment. Generally, the non-health care costs account for the largest part in total PET costs. Healthcare costs per bite case were generally higher in the urban area than in the rural areas due to a higher application level of supplementary treatments such as tetanus antitoxins in addition to the basic wound care. The non-healthcare costs per sufficient treatment were higher in the rural districts than in the urban area as the distance traveled to the nearest health center was longer than in the urban area.

The largest portion of non-health care costs in the rural districts was attributed to the productivity losses due to the time spent on searching treatment. The number of days spent by victims in search of treatment ranged from 0 to 30 days per case with averages of 7.0, 9.0 and 8.4 days per bite case, and 8.5, 16.5 and 16.7 days per sufficient treatment in Bishoftu, Lemunabilbilo and Yabelo, respectively. Out of the total dog bite victims, about 35\% was at least once accompanied by an adult family member (older than fifteen years of age) to the health center. The opportunity costs of time spent seeking for treatment per bite case ranged from zero to 54 USD with averages of 7.6, 10.0, and 8.7 USD for the victims and 1.6, 2.4, and 3.0 USD for the caregivers in Bishoftu, Lemuna-bilbilo and Yabelo, respectively. However, per sufficient treatment, the average opportunity costs of time seeking for treatment were equal to 9.2, 18.4, 
18.1 USD for the victims and to 0.9, 3.5 and 4.1 USD for the caregivers in the same district order.

The number of days required to get a single PEP dose in the urban area was considered half a day, while this was about one day in rural areas. The average number of days spent and the related opportunity costs while seeking treatment per case were comparable across districts due to a lower proportion of victims that was seeking for medical treatment in rural areas.

Table 5.5 Average (min, max) treatment costs (in USD) per dog bite (including all bite cases) and per sufficient PEP treatment.

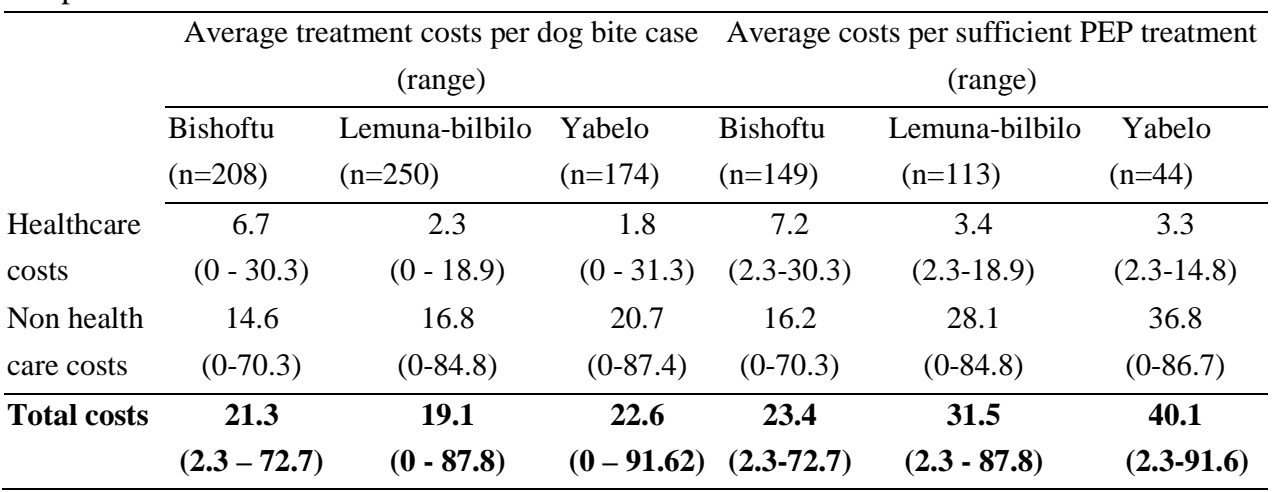

\section{ii. $\quad$ Health burden}

Based on the total of 465 rabies suspected dog bites, the numbers of deaths due to rabies disease out of 100,000 inhabitants in each district of Bishoftu, Lemuna-bilbilo and Yabelo were, respectively, 0.7, 4.2 and 2.9. The health burden, in terms of DALY, was corresponding to 19.4, 136.7 and 105.2 DALYs per 100,000 inhabitants per year under the age and time discounting scenario. The numbers of human deaths and DALYs were significantly higher in rural areas compared to the urban area ( $p<0.05$ ). Additionally, the estimated health burden due to the NTV application was equal to 2.7, 1.3, and 1.3 DALYs per 100,000 inhabitants per year in Bishoftu, Lemuna-bilbilo, Yabelo, respectively. The largest contribution to this health burden resulted from YLL (i.e. 99\%). The overall health burden due to rabies disease and NTV in Ethiopia was estimated to be 92.7 DALYs per 100,000 inhabitants per year (Table 5.6).

Table 5.6 Annual health burden due to rabies disease and adverse effects of Nervous Tissue Vaccine (NTV) in DALYs per 100,000 inhabitants.

\begin{tabular}{lllll}
\hline Source of burden & Overall & Bishoftu & $\begin{array}{c}\text { Districts } \\
\text { Lemuna-bilbilo }\end{array}$ & Yabelo \\
\hline Rabies disease & 90.9 & 19.4 & 136.7 & 105.2 \\
NTV & 1.8 & 2.7 & 1.3 & 1.3 \\
\hline Total & 92.7 & 22.1 & 138.0 & 106.5 \\
\hline
\end{tabular}




\subsection{Discussion}

This study aimed to assess the burden of rabies in Ethiopia in terms of DALYs and PET costs while accounting for variation across different agro-ecologies. We estimated an average annual human mortality rate due to rabies between 0.7 (in Bishoftu and 4.2 (in Lemuna-bilbilo) cases per 100,000 inhabitants with exposure rates ranging from 19-137 per 100,000 inhabitants with the highest number in Lemuna-bilbilo. Our estimates on number of exposures and deaths were higher than previous national estimates (Deressa et al., 2013) consisting of 12 exposure cases per 100,000 inhabitants and 1.6 rabies deaths per 100,000 inhabitants, respectively. These estimates were calculated from exposure cases that were reported to health centers. Our exposure estimate was also higher than the estimate of 1-5 exposure rates per 100,000 inhabitants as made by Yibrah and Damtie (Yibrah and Damtie, 2015), which also mainly referred to officially registered rabies cases. Underreporting in Ethiopia primarily occurs due to the deep-rooted traditional practice of treating rabies by healers, which as such interferes with assessing the real magnitude of the problem (Deressa et al., 2010). In line with this, we found that about half of the bite victims we contacted in rural areas did not report to health centers but visited traditional or spiritual healers. In the urban area, this proportion was less than $4 \%$. This considerable difference between the rural and urban areas could also relate to other socio-economic factors including difference in knowledge level, accessibility to health centres or differences in the availability of financial means that call for further investigation in an attempt to improve the rate of sufficient PET in the rural areas.

The health burden of rabies in Ethiopia ranged from 19-137 DALYs in urban and rural areas with an average of 3 human deaths per 100,000 inhabitants, which is comparable to findings in countries in the region like Tanzania where 4.9 rabies caused mortalities led to 154 DALYs per 100,000 inhabitants (Cleaveland et al., 2002). A recent global rabies burden study (Hampson et al., 2015) reported that Ethiopia suffered the highest national health impact due to rabies among the SADC Southern African Development Community with 86.4 DALYs per 100,000 inhabitants or 73,390 DALYs nationally. Our study as well as the study in Tanzania (Cleaveland et al., 2002), didn't consider the temporary disability related to the time lived with severe open wounds which was given a disability weight of 0.005 (0.002-0.013) DALYs per patient (Murray et al., 2013). The health burden due to the adverse effects of NTV is equivalent to $1 \%$ of the total health burden due to rabies disease. This estimate is comparable with a recent study on the global burden of rabies by Hampson et al., (Hampson et al., 2015) reporting the neurological adverse effect constituting $0.8 \%$ of total rabies-related DALYs contributed by the 10 countries that reported to use NTV at the time of the study.

The health impact per 100,000 inhabitants and post-exposure treatment costs per sufficient treatment were estimated to be higher in rural districts. Consequently, the health and financial 
burden of rabies for rural victims were higher than the urban area, although the human rabies exposure rate to potential rabid dogs was higher in the urban district.

People in the age category between 15 and 29 years followed by children in the age between 5 and 14 years represented the largest groups among the rabid suspected dog bite cases. Children of 0-14 years of age had the highest probability of being bitten on the head/neck compared to other age groups. As acknowledged by WHO (Prüss-Ustün et al., 2003), the high life expectancy allocated to children and the elevated chance of exposure result in a higher burden of rabies in these age groups. Comparable proportions across age groups were reported by other studies for Northern Ethiopia (Mengistu et al., 2011) and countries in the region (Hampson et al., 2008).

In this study, the average treatment costs per dog bite case irrespective of treatment status were more or less comparable among the districts. However, the proportion of health costs and nonhealth costs varied among the districts due to the fact that a higher proportion of the victims in the urban area sought medical treatment and needed to travel a shorter distance towards the nearest health center than victims in the rural areas. Health costs would generally increase if rabies immunoglobulin would also be administered for those who received PET. This is rarely applied in Ethiopian situation but recommended to all severe bite cases described by a single or multiple transdermal bites, scratches or contamination of mucous membrane with saliva (i.e. licks) (WHO, 2002). The non-health care costs which include opportunity costs of productivity/time losses while seeking for treatment represented the most relevant costs for treatment. The non-health care costs varied across districts and were significantly higher in rural districts that in the urban districts. On average, the PET costs of rabies was equal to approximately $4 \%$ of the GDP per capita (CSA, 2012). Considering only out of pocket spending, i.e. expenditures without considering the opportunity cost of time spent looking for treatment, the economic burden of PET would reduce with 50\% to $2 \%$ of the GDP per capita, which is still a severe financial burden.

Application of the WHO recommended cell culture PEP by a 5-dose vaccine regimen (Rupprecht et al., 2010) instead of the currently applied 17 doses NTV, would result in considerably higher health care costs (average of 62.5 USD per five doses WHO-PEP) versus 2.3 USD per 17 doses NTV-PEP). The non-healthcare costs will, however, be reduced due to the reduction in number of doses and therefore the required number of visits to the health centers ( 5 visits versus 17 visits). An estimation of the non-health costs under the WHO regimen can be obtained from the average non-health care costs spent per PEP dose (i.e. treatment day) under the NTV regime. Accordingly, the average estimated PET costs of the WHO 5 dose regimen the average PET costs would be equal to 71.8, 71.9 and 74.7 per sufficient treatment in Bishoftu, Lemuna-bilbilo, and Yabelo, respectively, or approximately 3.5 times higher than the current 
average PET costs under NTV. This increase in costs is primary related to the difference in price of the imported cell culture based PEP and the locally produced NTV. Comparable costs were also reported in Tanzania for 5 doses of WHO recommended cell culture based PEP (Shim et al., 2009; Sambo et al., 2013). Currently, the Ethiopian government is working towards establishing and producing cell culture based PEP locally that could lower the health care costs (Hurisa et al., 2013). While the costs of cell culture vaccines are high, we only estimated a small saving of DALYs as a result of avoided adverse NTV effects; 2.7, 1.3 and 1.3 DALYs per 100,000 inhabitants per year in, respectively, Bishoftu, Lemuna-bilbilo, and Yabelo.

Extrapolation to the national level weighed for the proportions of inhabitants in urban and rural areas of Ethiopia indicated an annual average of 3,000 human deaths (i.e. 3 rabies deaths per 100,000 inhabitants) and 97,000 rabies-exposed persons (101 exposure cases per 100,000 inhabitants) treated at an average costs of 21 USD per case, causing 2 million USD treatment costs per year and a health loss of about 93,000 DALYs. The national estimate for DALY lost due to adverse effects of the NTV equals 1500 DALY per year which makes the total burden 94,500 DALY per year. The estimated national health impact in this study is comparable to the estimation for Ethiopia as part of the global canine rabies burden costs (Hampson et al., 2015). Although the extrapolation to the national scale requires various assumptions the obtained estimates provide an indication of the extent of health and economic burden in the country and strengthen initiatives towards controlling rabies.

In Ethiopia, priority has been given to diseases like Malaria, HIV/AIDS, and TB that attract funding for achieving Millennium Development Goals (Hotez and Kamath, 2009). In addition, these diseases are rated as top priority in the current Ethiopian Growth and Transformation Plan (GTP). Although rabies is not comparable with these diseases that cause very high health and economic burden, our findings demonstrated that rabies causes a health and economic hardship and with a high likelihood of fatality for exposed rural inhabitants calling for national and global attention. Similarly, a recent study in Ethiopia (Pieracci et al., 2016) prioritized rabies as the most important zoonotic disease in Ethiopia. However, in practice rabies control is often overlooked by both public health and veterinary authorities. Considering the goal set by WHO to eliminate dog-mediated human rabies by 2030 (WHO, 2016) and meeting the Millennium Development Goals list of combating disease burden and reducing extreme poverty, including rabies control into the Ethiopian Growth and Transformation Plan would be very beneficial.

Studies in sub-Saharan Africa countries, where rabies is a public health concern, show that investments made to vaccinate a sufficient proportion of dogs would likely minimize the health burden and expenditures related to PET (Lembo et al., 2010; Fitzpatrick et al., 2014; Bilinski, et al., 2016). Strategies to reduce human rabies in Ethiopia should, therefore, include mass dog vaccination. However, the implementation of a sufficient mass dog vaccination requires 
extensive knowledge of the dog population, the expected cost-effectiveness of vaccination measures and above all an integration of public health and veterinary authorities for a progressive evaluation of one-health effectiveness in real time (Kahn et al., 2010).

\subsection{Conclusion}

In conclusion, this study evaluated the health and economic burden of rabies in three districts representing geographical diversity assuming possible variation in human exposure rates. Extrapolation of the results to the national level demonstrated that rabies, on average, causes three deaths per year per 100,000 inhabitants at 101 exposure cases per year per 100,000 inhabitants, causing 2 million USD treatment costs per year. This causes a relevant health and economic burden for Ethiopia. Human exposure rates to potential rabid dogs were higher in urban than in rural districts but the health impacts and post-exposure treatment costs were higher in rural districts. Consequently, the health and financial burden of rabies for rural victims are substantially higher than for urban victims. Under the current NTV regimen, non-health care costs are the major contributor of PET costs. The obtained estimations of the burden of rabies to the Ethiopian society provide decision makers with insights into the potential benefits of implementing cost-effective interventions, like mass dog vaccination.

\section{Acknowledgements}

We would like to thank the dog bite victims and their families who participated in the field survey and the health extension workers who collected the data. 


\subsection{References}

Ali, A., Mengistu, F., Hussen, K., Getahun, G., Deressa, A., Yimer, E., \& Tafese, K. 2010. Overview of Rabies in and around Addis Ababa, in Animals Examined in EHNRI Zoonoses Laboratory Between, 2003 and 2009. Ethiopian Veterinary Journal, 14(2), 91-101.

Ayele, W., Fekadu, M., Zewdie, B., Beyene, M., Bogale, Y., Mocha, K., Egziabher, F. 2001. Immunogenicity and efficacy of Fermi-type nerve tissue rabies vaccine in mice and in humans undergoing post-exposure prophylaxis for rabies in Ethiopia. Ethiopian Medical Journal, 39(4), 313-321.

Bilinski, A. M., Fitzpatrick, M. C., Rupprecht, C. E., Paltiel, A. D., Galvani, A. P. 2016. Optimal frequency of rabies vaccination campaigns in Sub-Saharan Africa. Proceedings of the Royal Society B: Biological Sciences, 283(1842). doi:10.1098/rspb.2016.1211

Cleaveland, S., Fevre, E. M., Kaare, M., Coleman, P. G. 2002. Estimating human rabies mortality in the united republic of Tanzania from dog bite injuries. Bull World Health Org, 80.

Coetzer, A., Kidane, A. H., Bekele, M., Hundera, A. D., Pieracci, E. G., Shiferaw, M. L., . . . Nel, L. H. 2016. The SARE tool for rabies control: Current experience in Ethiopia. Antiviral Research, 135, 74-80. doi:10.1016/j.antiviral.2016.09.011

CSA. 2012. Household Consumption andExpenditure (HCE) Survey 2010/11. Analytical report. Addis Ababa,Ethiopia.

CSA. 2013. Population Projection of Ethiopia for All Regions At Wereda Level from 2014 2017. Federal Democratic Republic of Ethiopia Central Statistical Agency, Addis Ababa, Ethiopia.

Deressa, A., Ali, A., Bayene, M., NiwayeSelassie, B., Yimer, E., Hussen, K. 2010. The status of rabies in Ethiopia: A retrospective record review. Ethiopian Journal of Health Development, 24(2).

Deressa, A., Sefir, D., Mamo, H., Ali, A., Akililu, M., Beyene, M., Getahun, G. 2013. Rabies diagnosis and surveillance for the period 2010-2012 in Ethiopia. 11th SEARG meeting, Dar es Salaam 11-15 February 2013, South Africa.

EHNRI. 2012. Proceding of the national workshop on Rabies prevention and control in Ethiopia, Oct 18-19, 2012, Addis ababa.

Fitzpatrick, M. C., Hampson, K., Cleaveland, et al., S., 2014. COst-effectiveness of canine vaccination to prevent human rabies in rural tanzania. Annals of Internal Medicine, 160(2), 91-100.

Hampson, K., Coudeville, L., Lembo, T., Sambo, M., Kieffer, A., Attlan, M., . . . Cleaveland, S. 2015. Estimating the Global Burden of Endemic Canine Rabies. 
Hampson, K., Dobson, A., Kaare, M., Dushoff, J., Magoto, M., Sindoya, E., Cleaveland, S. 2008. Rabies exposures, post-exposure prophylaxis and deaths in a region of endemic canine rabies. PLoS Negl Trop Dis, 2(11), e339.

Hossain, M., Ahmed, K., Bulbul, T., Hossain, S., Rahman, A., Biswas, M., Nishizono, A. 2012. Human rabies in rural Bangladesh. Epidemiology and Infection, 140(11), 19641971.

Hotez, P. J., Alvarado, M., Basáñez, M.-G., Bolliger, I., Bourne, R., Boussinesq, M., . . . Budke, C. M. 2014. The global burden of disease study 2010: interpretation and implications for the neglected tropical diseases.

Hotez, P. J., Kamath, A. 2009. Neglected tropical diseases in sub-Saharan Africa: review of their prevalence, distribution, and disease burden. PLoS Negl Trop Dis, 3(8), e412.

Hurisa, B., Mengesha, A., Newayesilassie, B., Kerga, S., Kebede, G., Bankovisky, D., . . . Urga, K. 2013. Production of Cell Culture Based Anti- rabies Vaccine in Ethiopia. Procedia in Vaccinology, 7, 2-7. doi:10.1016/j.provac.2013.06.002

Jo, C. 2014. Cost-of-illness studies: concepts, scopes, and methods. Clinical and molecular hepatology, 20(4), 327-337.

Kahn, C., Line, S., Co, M. 2010. The Merck Veterinary Manual. A handbook of diagnosis, therapy, and disease prevention and control for the veterinarian. Eds: . Inc., New Jersey, USA, 905-908.

Knobel, D. L., Cleaveland, S., Coleman, P. G., Fèvre, E. M., Meltzer, M. I., Miranda, M. E. G., . . . Meslin, F.-X. 2005. Re-evaluating the burden of rabies in Africa and Asia. Bulletin of the World Health Organization, 83(5), 360-368.

Lea, R. A. 1993. World Development Report 1993:'Investing in Health'.

Lembo, T., Hampson, K., Kaare, M. T., Ernest, E., Knobel, D., Kazwala, R. R., . . . Cleaveland, S. 2010. The feasibility of canine rabies elimination in Africa: dispelling doubts with data. PLoS Negl Trop Dis, 4(2), e626. doi:10.1371/journal.pntd.0000626

Ly, S., Buchy, P., Heng, N. Y., Ong, S., Chhor, N., Bourhy, H., Vong, S. 2009. Rabies situation in Cambodia. PLoS Negl Trop Dis, 3(9), e511.

Mathers, C. D., Vos, T., Lopez, A., Salomon, J., Ezzati, M. 2001. National burden of disease studies: a practical guide. Geneva: World Health Organization.

Mengistu, F., Hussen, K., Ali, A., Getahun, G., Sifer, D. 2011. Dog bite as a public health concern in Addis Ababa. Ethiopian Journal of Health Development, 25(1), 58-60.

Moges, N. (2015). Rabies in Ethiopia: Review Article.

Murray, C. (1994). Quantifying the burden of disease: the technical basis for disabilityadjusted life years. Bulletin of the World Health Organization, 72(3), 429.

Murray, C., \& Acharya, A. K. (1997). Understanding DALYs. Journal of health economics, 16(6), 703-730.

Murray, C., Lopez, A. D. 1996. Global burden of disease (Vol. 1): Harvard University Press Cambridge, MA. 
Murray, C. J., Ezzati, M., Flaxman, A. D., Lim, S., Lozano, R., Michaud, C., . . Vos, T. 2013. GBD 2010: design, definitions, and metrics. The Lancet, 380(9859), 2063-2066.

NBE. 2014. National Bank of Ethiopia, Exchange rates USD to EThiopian Birr on January 2014 (access date September 27, 2015).

Pieracci, E. G., Hall, A. J., Gharpure, R., Haile, A., Walelign, E., Deressa, A., . . Belay, E. 2016. Prioritizing zoonotic diseases in Ethiopia using a one health approach. One Health, 2, 131-135. doi:10.1016/j.onehlt.2016.09.001

Piyasirisilp, S., Hemachudha, T., Griffin, D. E. 1999. B-cell responses to myelin basic protein and its epitopes in autoimmune encephalomyelitis induced by Semple rabies vaccine. Journal of neuroimmunology, 98(2), 96-104.

Prüss-Ustün, A., Mathers, C., Corvalán, C., Woodward, A. 2003. Introduction and methods: assessing the environmental burden of disease at national and local levels (Vol. 1): OMS.

R Core Team. 2000. R language definition. Vienna, Austria: $R$ foundation for statistical computing.

Rupprecht, C. E., Briggs, D., Brown, C. M., Franka, R., Katz, S. L., Kerr, H. D., . . . Schaffner, W. (2010). Use of a reduced (4-dose) vaccine schedule for postexposure prophylaxis to prevent human rabies: recommendations of the advisory committee on immunization practices: Department of Health and Human Services, Centers for Disease Control and Prevention.

Sambo, M., Cleaveland, S., Ferguson, H., Lembo, T., Simon, C., Urassa, H., Hampson, K. 2013. The Burden of Rabies in Tanzania and Its Impact on Local Communities. Plos Neglected Tropical Diseases, 7(11), e2510.

Shim, E., Hampson, K., Cleaveland, S., Galvani, A. P. 2009. Evaluating the cost-effectiveness of rabies post-exposure prophylaxis: A case study in Tanzania. Vaccine, 27(51), 71677172. doi:10.1016/j.vaccine.2009.09.027

Taylor, L., Knopf, L. 2015. Surveillance of Human Rabies by National Authorities-a global survey. Zoonoses and Public Health, 62(7), 543-552.

Tenzin, T., Dhand, N. K., Gyeltshen, T., Firestone, S., Zangmo, C., Dema, C., . . W Ward, M. P. 2011. Dog bites in humans and estimating human rabies mortality in rabies endemic areas of Bhutan. Plos Neglected Tropical Diseases, 5(11), e1391.

Tepsumethanon, V., Wilde, H., Meslin, F. X. 2005. Six criteria for rabies diagnosis in living dogs. J Med Assoc Thai, 88(3), 419-422.

Teshome, E., Zenebe, M., Metaferia, H., Biadgilign, S. 2014. Participation and significance of self-help groups for social development: exploring the community capacity in Ethiopia. SpringerPlus, 3(1), 189.

WHO. (1984). WHO Expert Committee on Rabies. WHO Technical report series 709, Geneve, 1984. 
WHO. 2002. Current WHO GUIDE for Rabies Pre and Post-exposure Treatment in Humans Rabies Pre and Post-exposure Treatment in Humans. World Health Organization Department of Communicable Diseases Surveillance and Response. Last update: November 2002.

WHO. 2007. World Health Organization WHO Technical Report Series No 941.

WHO. 2013. Ethiopia life table estimate for 2013

(http://apps.who.int/gho/data/view.main.60550?lang=en. Accessed date 1/9/2015.

WHO. 2015. http://www.who.int/entity/healthinfo/bodreferencedalycalculationtemplate.xls accessed data 3/9/2015.

WHO. 2016. (World Health Organization) http://www.who.int/mediacentre/factsheets/fs099/en/ accesed date 15/4/2016.

WHO. 2017. Human rabies: 2016 updates and call for data. Wkly Epidemiol Rec, 92(7), 7786.

World Bank. 2015. GDP per capita for Ethiopia. http://data.worldbank.org/indicator/NY.GDP.PCAP.CD/countries/ET?display=graph access date- 3/9/2015.

Wunner, W. H., Jackson, A. C. 2010. Rabies: scientific basis of the disease and its management: Academic Press.

Yibrah, M., \& Damtie, D. (2015). Incidence of human rabies exposure and associated factors at the Gondar Health Center, Ethiopia: a three-year retrospective study. Infectious diseases of poverty, 4(1), 3. doi:10.1186/2049-9957-4-3

Yimer, E., Newayeselassie, B., Teferra, G., Mekonnen, Y., Bogale, Y., Zewde, B., . . Bekele, A. 2002. Situation of rabies in Ethiopia: a retrospective study 1990-2000. Ethiopian Journal of Health Development, 16(1), 105-112. 



\section{Chapter 6}

Determinants of health seeking behaviour following rabies exposure in Ethiopia

Tariku Jibat Beyene, Monique C. M. Mourits, Crawford W. Revie, Henk Hogeveen

Under review by Public health and Zoonoses journal 


\begin{abstract}
The objective of this study was to identify factors that determine medical treatment seeking behaviour following potential rabies exposure after being bitten by a suspected dog and the likelihood of compliance to receive sufficient doses of post-exposure prophylaxis after the visit to a health centre visit. An extensive survey based on case investigation was conducted on suspected rabid dog bite cases in three areas of Ethiopia. Two multivariable logistic regression models were created with a set of putative variables to explain treatment seeking and compliance outcomes. Among the putative factors tested, ownership status of the biting dog, severity of the bite, body part bitten, monthly spending (which was used as a proxy for income) and distance from health centre were found to be significant predictors of the likelihood of seeking medical treatment; while ownership status of the biting dog, monthly spending and distance to the health centre were found to be significant predictors of the likelihood of compliance in receiving sufficient doses of post-exposure prophylaxis. The overall likelihood of seeking medical services following rabies exposure was higher for people bitten by dogs of unknown ownership, where the bite was severe, being bitten on the leg, spend of more than 100 USD per month and where the victim lived close to the nearest health centre. Upon inclusion of district as an additional explanatory variable in the model, the effect of distance became less important while living in the urban district increased the likelihood of seeking treatment. In contrast, in the model to predict likelihood of compliance to treatment regimen, inclusion of district did not improve the model fit. Given the insights obtained from this study, improvements in the rural districts with regard to accessibility of post-exposure prophylaxis delivering health centres in shorter distance could improve health seeking behaviour.
\end{abstract}




\subsection{Introduction}

Neglected tropical diseases (NTDs) affect around a billion people each year globally and have been associated with an annual mortality of around 9 million people (Frew et al., 2009). These diseases occur primarily in rural and poor urban areas of sub-Saharan Africa, South Asia and Latin America (Hotez et al., 2007). NTDs are largely absent from industrialized countries and in wealthy societies within developing countries (Franka et al., 2013). This disproportionate burden of NTDs among the poor has been attributed to various socioeconomic, religious, demographic, geographic and disease related determinants (Bishwajit et al., 2014).

Rabies is considered to be one of these neglected tropical diseases, caused by a virus that affects all mammals including human beings (Garg, 2014). Domestic dogs serve as a reservoir and cause over $90 \%$ of all human deaths from rabies, while bats and wildlife contribute the remainder (WHO, 2005; Franka et al., 2013). Despite being a vaccine-preventable disease, rabies is still a significant public health problem in many developing countries within Asia and Africa (Sugiyama and Ito, 2007; WHO, 2013). Globally, canine rabies causes around 60,000 human deaths, over 3.7 million disability-adjusted life years (DALYs) and 8.6 billion USD economic losses annually. About $75 \%$ of these economic losses are due to premature death and costs associated with seeking treatment (Hampson et al., 2015).

Human infection can in principle be eliminated at the source through sustained mass vaccination of reservoir domestic dog populations. Once exposed by a bite from a rabid animal, human rabies can only be prevented through prompt post-exposure treatment, which includes wound washing, administration of an antibiotic, tetanus antitoxin (in severe bites) and postexposure prophylaxis (PEP) (Hemachudha et al., 2002; WHO, 2013). Otherwise, rabies is invariably a killer disease once clinical signs are manifested. This means that urgent postexposure treatment and prophylaxis are needed to reduce the likelihood of human mortality (Hemachudha et al., 2013).

In Ethiopia, a selected urban district, a rural highland district and a rural lowland district, the annual rabid dog exposures were estimated to be 135, 101 and 86 bites per 100,000 inhabitants, respectively (Beyene et al., 2017). That study revealed that $71 \%$ of bite victims reported to health centres seeking medical service upon exposure, while only $57 \%$ of the bite victims received sufficient doses of PEP. At the district levels, 97\%, 64\%, and 66\% of the victims reported to health centres seeking medical treatment, while $78 \%, 50 \%$, and $29 \%$ received sufficient doses of PEP in urban, rural highland and rural lowland districts respectively. In Ethiopia, a complete PEP treatment consists of 17 doses of a nervous tissue vaccine, administered consecutively during the first 14 days after exposure, with the remaining 3 doses being given at intervals of 10 days; i.e. at Day 24, 34 and 44. 
Most of the studies concerning rabies in Ethiopia have focused on levels of public awareness, attitudes and practices in cases of rabies exposure (Digafe et al., 2015; Ramos et al., 2015; Kabeta et al., 2015; G/hiwot et al., 2016; Jemberu et al., 2013; Yibrah and Damtie, 2015; Teklu et al., 2017). None of these studies specifically identified factors which explain the medical treatment seeking behaviour or the likelihood of receiving sufficient treatment doses among affected individuals. It is well understood that whilst such studies are helpful to understand the current levels of knowledge and practice, they are typically not able to infer causal associations or predict the consequence of particular actions taken. Understanding these factors is essential to support decisions in mitigating the hindrances that could lead to a reduced burden of rabies.

The decision to engage with medical channels following exposure or illness is influenced by a variety of geographical, social, economic, cultural and organizational factors (Tipping and Segall, 1995). A range of these factors including, age (Chibwana et al., 2009), gender and education level (Mwangome et al., 2010), cultural beliefs and religion (Feyisetan et al., 1997), fate of person or animal responsible for transmitting the disease (Tepsumethanon et al., 2005), were explored in the current study in terms of their effect on the decision to seek treatment. Specific to rabies, the severity of bite and the body part bitten have been shown to affect the likelihood of rabies disease development (Knobel et al., 2005). Source of income for treatment and knowledge about the vaccination status of the biting dog (Sambo et al., 2013) have also been described as factors influencing an individual's decision to seek medical treatment upon rabies exposure. Anthropological studies have also shown that medical treatment seeking behaviours within communities in developing countries depend on the accessibility of health facilities (Kroeger, 1983; Shaikh and Hatcher, 2004; Bichmann et al., 1991). Yet, there is not much knowledge about health seeking behaviour after dog bites in Ethiopia and about the differences between rural and urban areas.

Thus, the objective of this study was to identify factors that determine the likelihood of seeking medical treatment behaviour at a health centre following rabies exposure and the likelihood of compliance to the required PEP treatments in different districts in Ethiopia.

\subsection{Materials and methods}

\subsubsection{Study design}

An extensive survey based upon case investigation was conducted. Suspected rabid animal bite cases were identified in three districts of Ethiopia, namely: Bishoftu, Lemuna-bilbilo, and Yabelo. These districts represent urban, rural highland and rural lowland areas with populations of 140,039, 187,222 and 100,737 inhabitants, respectively (CSA, 2013). Bishoftu is an urban district $45 \mathrm{~km}$ southeast of Addis Ababa, the capital of Ethiopia. Lemuna-bilbilo is a rural district located in the central highlands of Ethiopia where most people adopt a mixed crop- 
livestock farming system. Yabelo is a district in the lowlands of the southern part of Ethiopia where the majority of people are pastoralists. In Ethiopia, each district has at least one health centre delivering human post-exposure prophylaxis services to individuals exposed to rabies.

Data on animal bite victims who visited health centres after being bitten by an animal in the period between September 2013 and August 2014 were collected from the records of health centres in the three districts. Local health extension workers practicing in each village of the districts were trained on techniques to carry out interviews using a structured questionnaire as well as in searching for cases that were not reported to health centres, using the contact tracing method described in our previous work (Beyene et al., 2017). The questionnaire was pre-tested with 30 bite victims, 10 in each of the three districts. Based on these pre-tests, the questionnaire was adjusted mainly for language and clarity. The list of bite cases collected from the health centres was then sorted into victim's village and given to local health extension workers. A local health extension worker went to each victim's house, asked for and confirmed the victim's oral consent to participate in the study, and then interviewed them using the structured questionnaire. In cases where the victim was deceased or was a child, an adult family member was interviewed.

The questionnaire contained sections on: (1) demographics (age, sex, educational level), adulthood (if someone is older than fifteen years of age s/he was considered responsible for the decision to visit a health centre, otherwise s/he was assumed to be cared for by other family members) and religion of the victim; (2) average monthly spending of the victim (as a proxy for monthly income) (Uzochukwu and Onwujekwe, 2004); (3) characteristics of the bite in terms of severity (classified according to WHO categories for exposure (WHO, 2013)) and body part bitten; (4) behavioural manifestations, ownership, and fate of the biting animal; 5) linear distance to the nearest health centre that could deliver PEP, (6) whether the victim sought medical or traditional treatment or none at all, and (7) if medical treatment was sought, the number of PEP doses subsequently received. The health extension workers also asked the bite victims and their families whether they knew of anyone who had been bitten by an animal within their community but reported this to another health centre or went to a traditional/spiritual healer or did nothing. Subsequently, any potential victims identified in this manner were contacted and interviewed using the same structured questionnaire (Beyene et al., 2017).

\subsubsection{Statistical analyses}

The 655 collected questionnaires were checked for completeness and then entered into Microsoft Excel. Of these 655 cases, 23 were due to bites from animals other than dogs. Because of their low number, these bite cases were excluded from further analyses. The behavioural manifestation and signs of the biting dogs as described by the bite victims were used to categorise each dog as potentially rabid or non-rabid, based on the "six-step" method 
(Tepsumethanon et al., 2005). Only bite cases by dogs fulfilling all six criteria were retained for further analyses, leaving in total 465 observations of exposures by suspected rabid dogs.

Descriptive statistics (counts and means) were used to describe the bite victims' sex, religion, adulthood, educational level, dog ownership, the severity of the bite, body part bitten, fate of the biting dog, monthly spending, places sought for treatment (medical health centre, traditional/spiritual, nowhere), and sufficiency of PEP doses received, by district and overall. In cases where multiple parts of the body were bitten, the part of the body nearest to the head was used for categorisation (Knobel et al., 2005). Chi-square tests were used to test for significant differences between proportions in categorical groups. One-way ANOVA and pairwise posthoc comparison using Tukey's HSD test were carried out on continuous variables (victim's age and distance from the nearest health centre) across the three districts.

To explore factors that predict the likelihoods of 'a health centre visit' and 'receiving sufficient doses of PEP', two separate multivariable logistic regression models were built. In the first model the outcome variable was defined as follows: a victim bitten by a suspected rabid dog either visited a health centre or did not. In the second model the outcome variable was defined as: given that the victim visited a health centre, either s/he received the minimum required PEP doses or did not. A treatment consisting of at least 14 of the advised 17 PEP doses was considered to be "sufficient", while any treatment consisting of less than 14 doses was considered to be "insufficient", based on the minimum required number of doses to produce the neutralizing antibody level (WHO, 1984; Piyasirisilp et al., 1999; Ayele et al., 2001).

Potential determinants for the likelihoods of seeking medical treatment following rabies exposure and of compliance to the treatment regimen were derived from literature (Tipping and Segall, 1995; Chibwana et al., 2009; Mwangome et al., 2010; Feyisetan et al., 1997; Tepsumethanon et al., 2005; Knobel et al., 2005; Sambo et al., 2013). An important question to explore is whether these general determinants are able to explain the observed differences among districts in terms of health seeking behaviour (Beyene et al., 2017) or whether some differences are caused by district specific features. In order to evaluate each dependent variables, two models were created. The first was using a set of 11 independent predictor variables namely: age, sex, educational level, adulthood, religion, monthly spending, ownership of biting dog, the severity of dog bite, body part bitten, the fate of the dog after bite, and distance of the village from the nearest health centre delivering PEP services. The second model was using the set of 11 aforementioned variables to which a district variable was added to account for district-specific features.

As a first step, the association between the candidate variables and the outcome variables were evaluated using univariable logistic regression. Any variable that had a p-value of $<0.20$ in this 
univariable analysis was considered for inclusion in the multivariable logistic regression model. Next, the candidate variables were checked for collinearity by means of variance inflation factors (VIF). Multicollinearity was considered to be present if VIF > 10 (Dohoo et al., 2003) for any pair. Since all VIF values were $<2.1$ all candidate variables were explored during the multivariable logistic regression modelling process. The final models were built using backward stepwise elimination of those variables which had a p-value greater than 0.05 . The generic final multivariable logistic regression models had the following form:

$\ln [Y /(1-Y)]=\beta_{0}+\beta_{1} X_{1}+\beta_{2} X_{2}+\beta_{3} X_{3}+\ldots+\beta_{\mathrm{p}} \mathrm{X}_{\mathrm{p}}$

where $\ln [\mathrm{Y} /(1-\mathrm{Y})]$ is the $\log$ of the odds of the outcome (e.g. visiting a health centre or not; represented by (Y) and (1 - Y), respectively), the constant $\beta_{0}$ is the estimated intercept, while $\beta_{1}, \beta_{2}, \beta_{3}, \ldots, \beta \mathrm{p}$ represent the regression coefficients for each predictor variable. Exponentiation of the relevant regression coefficient $\left(\mathrm{e}^{\beta 1, \ldots, \beta \mathrm{p}}\right)$ provides the odds ratio (OR) for each of the predictor variables (Ott \& Longnecker, 2008).

The goodness of fit of the final multivariable logistic models was assessed using the Hosmer and Lemeshow test, where $\mathrm{p}$ - values higher than 0.05 indicating no evidence of poor fit), while their predictive power was assessed by means of the Pseudo $\mathrm{R}^{2}$ measure (McFadden, 1978). All statistical analyses were performed using the R software package, Version 3.2.1 (R Core Team).

\subsection{Results}

\subsubsection{Rabies exposures}

In total 655 victims bitten by animals between Sept 2013 and August 2014 were found and interviewed with 210 cases in the urban district (Bishoftu), 262 cases in the rural highland district (Lemuna-bilbilo), and 183 cases in the rural lowland district (Yabelo). We identified a total of 465 suspected rabid dog bite cases of which 189, 189 and 87 cases were from Bishoftu, Lemuna-bilbilo and Yabelo districts respectively. Overall, out of the 465 people bitten by a suspected rabid dog, 360 (77.4\%) visited a health centre, while 267 (57.4\%) of the exposed persons received sufficient doses of PEP. The proportions in terms of type of treatment sought and doses of PEP received differed across evaluated districts $(\mathrm{P}<0.01)$ (Table 6.1). 
Table 6.1 Description and comparisons on types of treatment sought, and level of PEP treatment by district and overall.

\begin{tabular}{|c|c|c|c|c|c|c|c|c|}
\hline \multirow[t]{2}{*}{ Variables } & \multicolumn{2}{|c|}{ Bishoftu } & \multicolumn{2}{|c|}{$\begin{array}{l}\text { Lemuna- } \\
\text { bilbilo }\end{array}$} & \multicolumn{2}{|c|}{ Yabelo } & \multicolumn{2}{|l|}{$\begin{array}{l}\text { Ove } \\
\text { rall }\end{array}$} \\
\hline & $\mathbf{n}$ & $\begin{array}{r}\text { p- } \\
\text { value }^{1}\end{array}$ & $\mathbf{n}$ & $\begin{array}{r}\text { p- } \\
\text { value }^{1}\end{array}$ & $\mathbf{N}$ & $\begin{array}{r}\text { p- } \\
\text { value }^{1}\end{array}$ & $\mathbf{N}$ & $\begin{array}{l}\text { p- } \\
\text { value }^{2}\end{array}$ \\
\hline Potentially rabid cases (n) & 189 & & 189 & & 87 & & 465 & \\
\hline Treatment types & & $<0.01$ & & $<0.01$ & & $<0.01$ & & $<0.01$ \\
\hline Medical by health centre & 183 & & 120 & & 57 & & 360 & \\
\hline Traditional & 5 & & 67 & & 27 & & 99 & \\
\hline $\begin{array}{r}\text { Neither medical nor } \\
\text { traditional }\end{array}$ & 1 & & 2 & & 3 & & 6 & \\
\hline Sufficient doses of PEP & & $<0.01$ & & $<0.01$ & & $<0.01$ & & $<0.01$ \\
\hline Did not visit centre & 6 & & 69 & & 30 & & 105 & \\
\hline Insufficient & 36 & & 18 & & 31 & & 85 & \\
\hline Sufficient & 147 & & 95 & & 25 & & 267 & \\
\hline NA & 0 & & 7 & & 1 & & 8 & \\
\hline
\end{tabular}

${ }^{1}$ Chi-square (P value) comparison across categories within district

${ }^{2}$ Chi-square (P value) comparisons across categories between districts

\subsubsection{Characteristics of variables across rabid dog bite victims}

Table 6.2 compares the socioeconomic, demographic, geographic and exposure-related factors between variables categories within districts and across districts. There were no statistical difference between proportions of categories across the three districts for the variables adulthood $(\mathrm{p}=0.20)$, monthly spending/income $(\mathrm{p}=0.1)$, body part bitten $(\mathrm{p}=0.79)$ and mean age at exposure $(\mathrm{p}=0.67)$. The mean age of the bite victims was 23 years (range: $1-85$, median $=19$ years). There were significantly higher bite cases in males (259/465) than in females (206/465) ( $\mathrm{p}$ < 0.05), but female exposures tended to be higher than male exposures in Bishoftu district. The monthly spending, which was assumed to be a proxy for income, was found to be significantly different across categories within the same district as well as across districts $(\mathrm{p}<$ 0.01). The highest bite rates were recorded on the leg/thigh (324), followed by hands/arm (88), trunk (27) and head/neck (26). The majority of the bites were single bites, though multiple bites of the hand or leg were recorded in $13.5 \%$ of the cases. The average distance to the nearest health centre delivering PEP was 15 km, while 2.5 (0.4-4) km, 18.4 (3-60) km, and 35.7 (0.5$70) \mathrm{km}$ were the averages for the urban, rural highland and rural lowland districts, respectively. 
Table 6.2 Description and comparisons on socioeconomic, demographic, geographic and exposure-related factors explaining likelihoods of visiting health centre and compliance with receiving sufficient doses of PEP upon potential rabies exposure by district and overall.

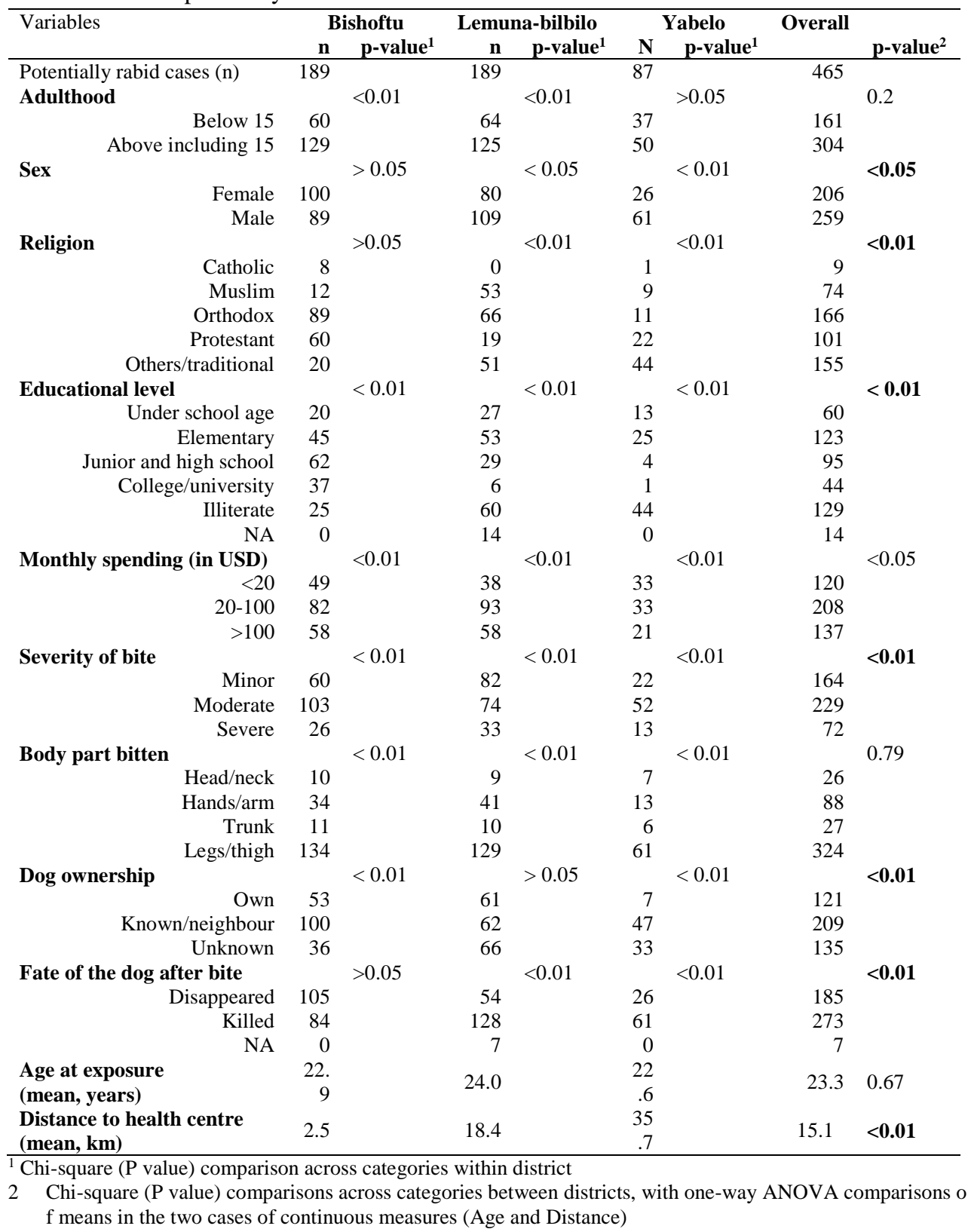


Predictors of health seeking behaviour after suspected rabid dog bite

Out of the total 465 suspected rabid dogs, 14 entries were missed a value for the variable "Educational level" while a further 7 entries were missing data from the variable "Fate of the biting dog". Thus the logistic model for the likelihood of seeking treatment at a health centre was based on 444 observations. Out of the first set of eleven variables considered as putative determinants of whether a visit would be made to a health centre after exposure to a suspected rabid dog, the univariate logistic regression analysis indicated no significance for the variables: age, adulthood, and religion ( $\mathrm{p}>0.2$ ). Subsequent analysis of the remaining eight variables using multivariable logistic regression identified only four significant $(p<0.05)$ variables, namely: ownership of the biting dog, the severity of the bite, body part bitten, monthly spending and straight line distance to the nearest health centre delivering PEP. The final multivariable logistic regression model indicated that victims bitten by a suspected dog with 'unknown' ownership status were more likely $(\mathrm{OR}=2.40,95 \% \mathrm{CI}=1.22-4.83)$ to visit a health centre compared to those bitten by a dog that was considered to be their 'own'. Victims of 'severe' and 'moderate' dog bites were also more almost two times likely to visit a health centre than people with 'minor' bite. Victims bitten on the 'head/neck' were less likely (OR $=0.35,95 \%$ $\mathrm{CI}=0.14-0.95)$ to visit a health centre compared to those bitten on the 'leg'. Victims with higher monthly spending (> 100 USD) were also found to be around two times more likely $(\mathrm{OR}=2.06,95 \% \mathrm{CI}=1.08-4.03)$ to visit health centre compared to those spend a lower amount ( $<20$ USD) of monthly spending. Distance to the health centre was shown to be negatively correlated to the likelihood of a visit to the health centre ( $\mathrm{OR}=0.96,95 \% \mathrm{CI}=0.94-0.97)$. The shorter the distance to the nearest health centre the higher the likelihood that a visit would be undertaken. For instance, a 1 KM shorter distance to a health centre increased the likelihood of visiting the health centre by $4 \%(1 / 0.96)$. The fitted multivariable model had acceptable goodness of fit (a Hosmer and Lemeshow goodness of fit p-value of 0.18 ) but low predictive value (a McFadden Pseudo $\mathrm{R}^{2}$ of 0.12) (Table 6.3).

After including district as an explanatory variable in the model, the variables ownership of the biting dog and severity of the bite were still significant; however, monthly spending, body part bitten and distance to the nearest health centre were no longer significant ( $p>0.05$ ). The added district variable was highly significant $(p<0.01)$; that is, those living in an urban district (Bishoftu) were much more likely to visit a health centre compared to those living in rural districts (both in Lemuna-bilbilo and Yabelo, with an $\mathrm{OR}=0.06,95 \% \mathrm{CI}=0.02-0.19$ ). The model fit indices after the inclusion of the district variable improved significantly as indicated by a Hosmer and Lemeshow goodness of fit p-value of 0.46 and a McFadden Pseudo $\mathrm{R}^{2}$ of 0.22 (Table 6.3). 


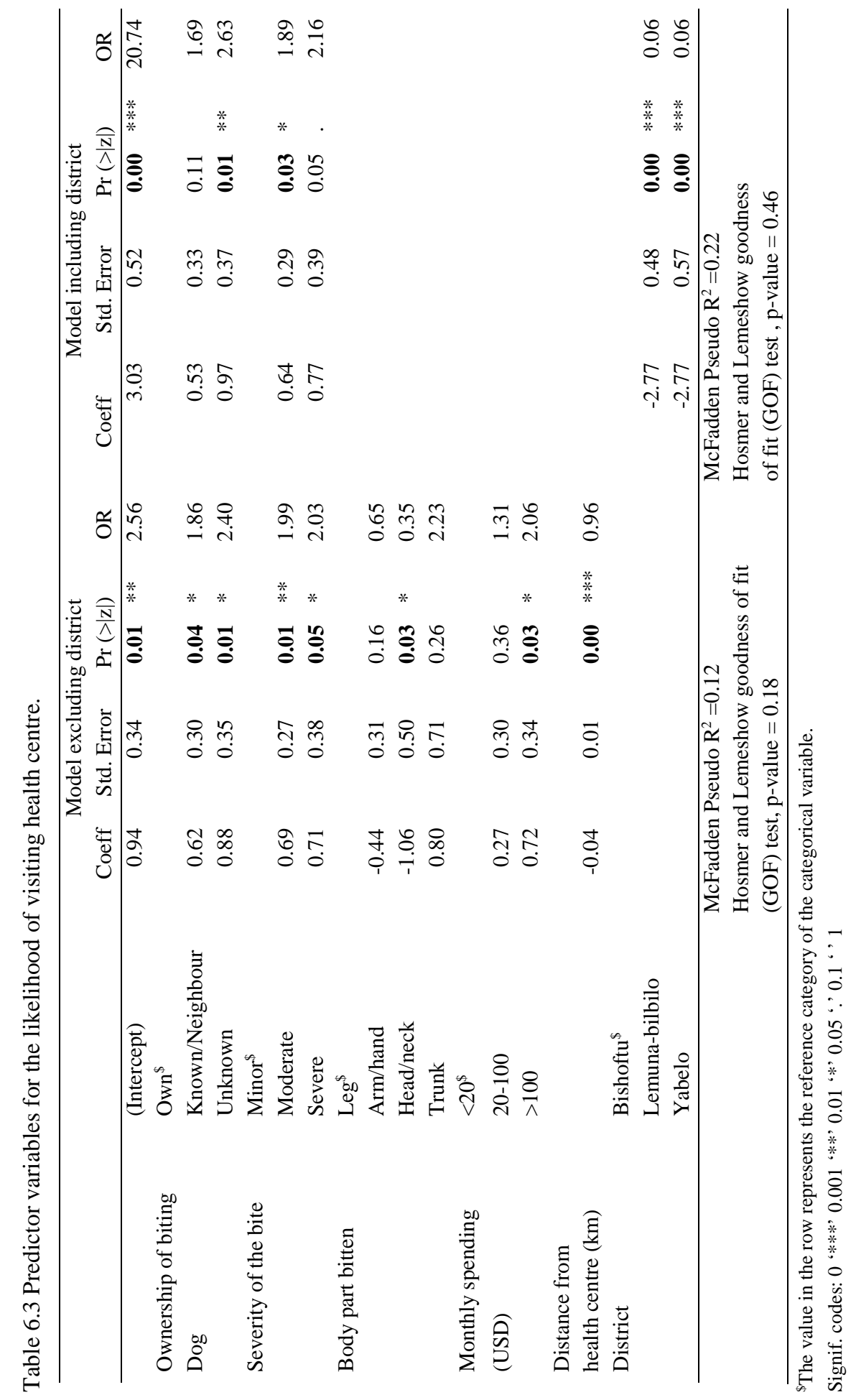


Predictors of likelihood of receiving sufficient doses of PEP after a suspected rabid dog bite About 77.4\% (360/465) of the suspected rabid dog bite victims visited a health centre. After excluding cases with missing values, data on 342 bite victim observations were used to identify determinants of likelihood to receive sufficient doses of PEP. Out of the set of 11 predictive variables as run in the first model, the univariate logistic regression analysis revealed no significant effect on the likelihood of receiving sufficient PEP for the variables: sex, religion, adulthood, fate of the biting dog, or body part bitten ( $p>0.2$ ). The inclusion of the remaining six variables in the multivariable logistic regression model, indicated that only monthly spending and distance to the health centre were significant determinants of the likelihood of receiving sufficient doses of PEP $(\mathrm{P}<0.05)$ (Table 6.4). Victims living in villages further away from the health centre were less likely to receive a sufficient dose of PEP (OR $=0.96,95 \% \mathrm{CI}$ $=0.95-0.98)$ i.e. for every one $\mathrm{km}$ distance closer to the health centre, the likelihood of completing the PEP treatment increased by 4\% (1/0.96). Victims with higher monthly spending ( $>100$ USD) were also found to be almost three times more likely (OR=2.72, 95\% CI $=1.33$ 5.71) to receive sufficient doses of PEP compared to those having a low level of monthly spending (< 20 USD). The multivariable logistic regression model was found to have a poor goodness of fit (Hosmer and Lemeshow p-value of 0.00) as well as limited predictive power (McFadden Pseudo $\mathrm{R}^{2}$ of 0.08 ).

When the multivariable model was extended to include the district variable, ownership of the biting dog became significant predictors $(\mathrm{p}<0.05)$. There were also significant differences among districts. Victims who lived in the Lemuna-bilbilo district had the same likelihood of receiving sufficient PEP as those living in Bishoftu. Living is Yabelo district was, however, linked to a significantly lower likelihood of receiving sufficient PEP when compared to the other two districts ( $\mathrm{OR}=0.37,95 \% \mathrm{CI}=0.14-0.96)$ i.e. victims from Bishoftu or Lemuna-bilbilo were three times more likely to complete the PEP treatment compared to victims from Yabelo. The model fit indicated no improvement with a Hosmer and Lemeshow goodness of fit p-value of 0.00 , while the McFadden Pseudo $\mathrm{R}^{2}$ increased to 0.12 (Table 6.4). 


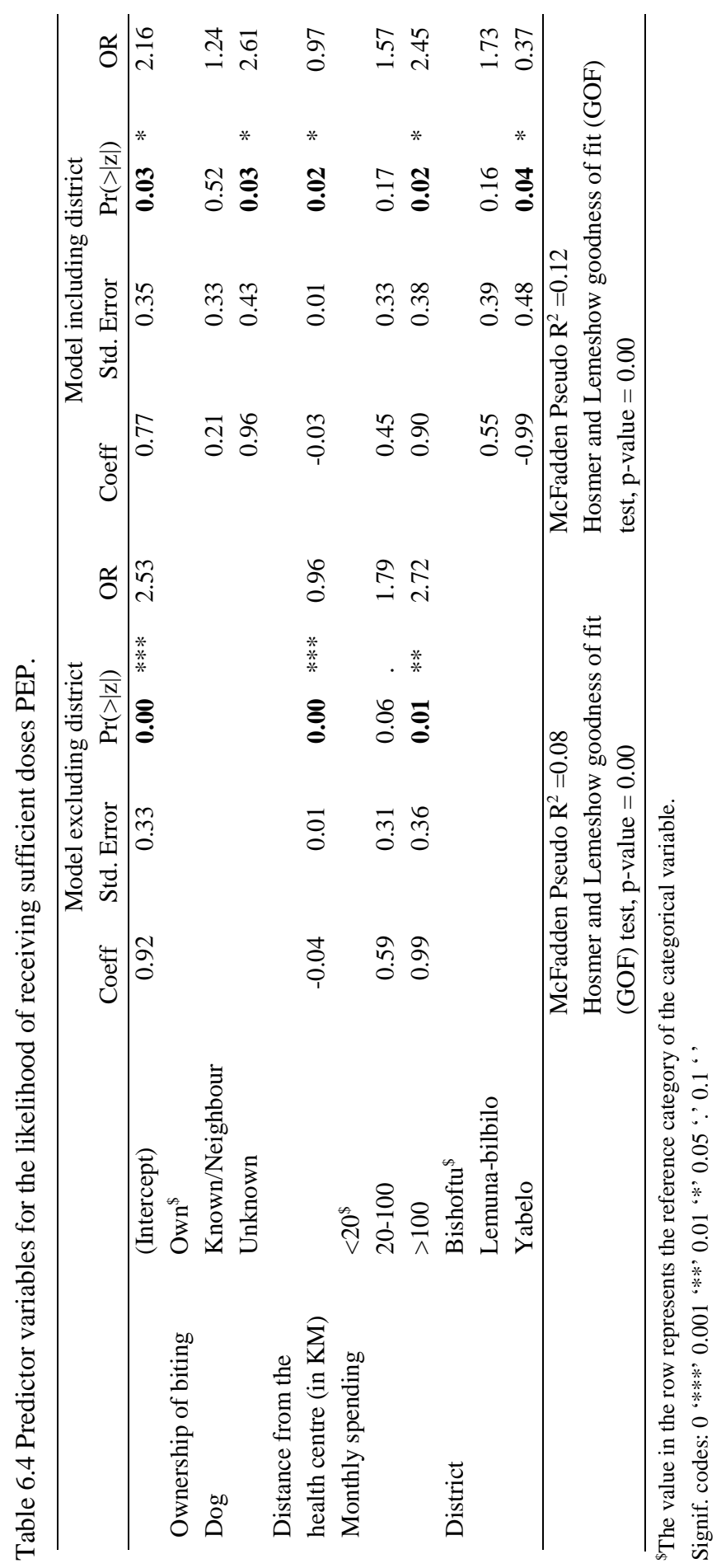




\subsection{Discussion}

Our data indicate that not everyone exposed to rabies seeks medical treatment, nor do those who do necessarily comply with recommended treatment doses. Consequently, rabies caused 1, 4 and 3 human deaths per 100,000 inhabitants in Bishoftu, Lemuna-bilbilo and Yabelo districts (Beyene et al., 2017) during the year under study. Therefore, identification of factors influencing medical treatment seeking behaviour is important in generating evidence that supports decision making to mitigate the hindrances, which will ultimately lead to a reduced health burden due to rabies. For illnesses such as rabies that require swift attention, reduction of complications that arise from delays in seeking medical care can be addressed by identifying factors that guide intervention (Mackian et al., 2004).

In this study the selection of relevant factors that might explain health seeking behaviour (i.e. sex, age, educational level, adulthood, religion, monthly spending as proxy of income, severity of bite, body part bitten, ownership and fate of the biting dog, and distance from the nearest health centre delivering PEP) was based on literature findings (Chibwana et al., 2009; Mwangome et al., 2010; Feyisetan et al., 1997; Tepsumethanon et al., 2005; Knobel et al., 2005; Sambo et al., 2013; Kroeger, 1983; Shaikh and Hatcher, 2004; Bachmann et al., 1991). Of these factors, ownership status of the biting dog, the severity of the bite, body part bitten, monthly spending and the distance to the nearest health centre delivering PEP were found to significantly influence the likelihood that a victim would visit a health centre after being bitten by a suspected rabid dog.

Being bitten by a dog of "unknown" ownership more than doubled the likelihood that a victim would visit a health centre. It is intuitive that people bitten by a dog of unknown ownership are less confident about the history and health status of the dog compared to a bite from a dog that they own or of one owned by their neighbour of whom they could make an enquiry regarding, for example, vaccination status. In the cases where victims received either 'severe' or 'moderate' grade bites they were around twice as likely to visit a health centre visit compared to those receiving only 'minor' grade bites. This practice should be encouraged as bites graded as 'severe' create a favorable environment for the rabies virus to penetrate the skin barrier and increase the likelihood of developing the disease. Therefore, the World Health Organization recommends immediate medical attention that includes administration of rabies immunoglobulin for severe bites. Such wounds are also liable to other microbial infection and often require antibiotic treatment and tetanus antitoxin (WHO, 2016). The fact that 'moderate' bites were also much more likely to be associated with a health centre visit could also be seen as a positive outcome particularly in terms of public awareness, as a 'better safe than sorry' policy makes good sense if there is any doubt as to the severity of the bite. 
Differing from our expectations, based on the perceived high risk of developing rabies when a bite is made to the head/neck, and in contrast to findings elsewhere (Knobel et al., 2005; Cleaveland et al., 2002; Shim et al., 2009), it appeared that being bitten on the head/neck was actually associated with a lower likelihood $(\mathrm{OR}=0.35)$ that a visit would be made to a health centre when compared to a bite on the leg. However, the contribution of head/neck bites to the total number of cases was minor (5.7\% of the total exposure cases) and as such is rather sensitive to small changes in number of data entries.

The logistic regression model predicting that the likelihood that a visit would be made to a health centre reduced by $4 \%$ for each additional $\mathrm{km}$ of distance from the victim's place of residence. Reports in the literature have also noted geographic accessibility, in terms of the physical distance or travel time to the health services delivery point, to be a major hindering factor to accessing health care in developing countries (Peters et al., 2008; Sambo et al., 2013).

Interestingly, upon inclusion of district as a variable in our models, the predictive effect of distance to the nearest health centre became non-significant. In addition, the goodness of fit, as well as the predictive power of the model improved, indicating that the district-specific information led to more robust model. This could potentially be due to the fact that variables specific to a district such as the availability of traditional healers, attitudes towards PEP treatment, and knowledge about rabies vary among districts and were not captured in the other specific case-level variables. Traditional and spiritual treatments against rabies are generally more common in rural than in urban communities (Tolossa et al., 2013; Digafe et al., 2015; Kabeta et al., 2015; Teklu et al., 2017). This difference was also found in our study. However, as only minor variations were seen in the proportion of followers of different religions across districts, visiting a traditional healer can, most probably, be seen to be a cultural rather than a religious practice.

People in urban areas have better access to mass media such as television, radio, and newspapers, which could increase their awareness about the disease and the necessity of seeking medical treatment subsequent to possible exposure. Infrastructures such as road and transportation facilities from rural areas to health centres delivering PEP will also tend not to be as developed as is the case in urban areas. Furthermore, the perceived effectiveness of rabies treatment by people from rural areas may be lower as these people tend to arrive at health centres later than is the case for those in urban areas, due to the longer distances and reduced transportation facilities. This could lead to a higher likelihood that rabies will develop, despite the PEP treatment (WHO, 2016).

The variables monthly spending and distance to health centre were found to significantly influence the likelihood of receiving sufficient doses of PEP. This indicates that once a victim 
has visited a health centre to seek medical treatment, a shorter distance to that centre, and a higher level of monthly spending (measured as a proxy for income) remain factors that increase the likelihood of compliance with the PEP regimen. The inclusion of the district variable did not have such a marked effect on model performance for this outcome, though the effect of distance was reduced, while ownership of the biting dog became significant. In contrast to the results for likelihood of seeking medical treatment, we found difference between the two rural districts, Lemuna-bilbilo and Yabelo, in terms of likelihood of receiving sufficient doses of PEP. Victims from Lemuna-bilbilo demonstrated similar likelihoods to those from the urban district (Bishoftu) and were found to be 3 times more likely to receive sufficient doses of PEP than were those from Yabelo. The difference between these two rural districts in terms of completion rates may be explained by differences in the means of livelihoods. In the pastoral rural lowland district people move often in search of feed and water for their livestock, while in the rural highlands people are relatively permanently resident, growing crops and keeping livestock in permanent place (Desta \& Coppock, 2004). The mobile nature of victims in the rural lowlands may have reduced the likelihood that they would receive sufficient doses of PEP.

Increasing the coverage of PEP delivering services by involving private health centres may improve medical treatment seeking and PEP compliance rates (personal communication with Dr. Abraham Haile Kidane, head of zoonosis research at the Ethiopian Public Health Institute), mostly by reducing the average distance that a victim would need to travel to receive medical treatment and hence of the time to the start of treatment.

In the present study, the highest level (>100 USD) of monthly spending, which was used as a proxy for income, was found to double the odds of visiting a health centre; however, it tripled the odds of PEP compliance compared to the lowest level (<20 USD) of monthly income. Before a victim visits a health centre upon exposure, s/he may not be aware that at least 14 doses of PEP are required as part of the treatment (EHNRI, 2012). Visiting a health centre with such frequency may be economically challenging, as the average total cost of post-exposure treatment is comparable to $4 \%$ of the average annual Ethiopian income, which would explain the influence of income level on the odds of treatment compliance. Out of the total costs associated with post-exposure treatment, non-health related expenses (mainly travel and time) contribute up to $70 \%$ of the total cost. Relaxation of the non-health related expenses could be achieved by introducing the 5 dose WHO-recommended PEP vaccine. However, as the WHOrecommended vaccine is more expensive than the nervous tissue vaccine which is currently used, the total costs would be around three times higher (Beyene et al., 2017).

Besides the variables we tested, length of treatment days which is 14-17 doses in medical treatment as opposed to 1-3 days for traditional/spiritual treatment and vaccine availability at the time of visit, may also have influenced/impacted the likelihood of seeking medical treatment 
and receiving sufficient doses of PEP (Deressa et al., 2010; Ramos et al., 2015). However, due to the lack of relevant records, it was not possible to retrospectively check vaccine availability for the times at which the victims sought treatment in the various health centres. It was also observed that a higher proportion of potential cases were deemed to be by rabid dogs in Bishoftu than was the case in Yabelo. It seems unlikely that this difference was due purely to chance and could be due to the level of exposure to unfriendly or aggressive biting dogs (those not under ownership) but still not rabid while evaluated based on the six criteria for assessing the rabid status retrospectively. For instance, the proportion of bites from dogs of unknown ownership in Yabelo and Lemuna-bilbilo (rural districts) was much higher than was the case for bites in Bishoftu (urban district).

In this study, we identified important factors influencing people's medical treatment seeking and treatment regimen behaviour. However, the factors we considered only partly explain the variability in the data, especially with respect to the likelihood of treatment compliance. A part of the unknown factors is linked to district, especially for the probability to visit a health centre. Since most socio-demographic variables that are known from literature were accounted for in this study, the factors that might explain the likelihoold to go to a health centre or to complete the treatment might be sought in perceived psychological factors, such as attitude or social norms. Further exploration of these factors can be done using methods like the theory of planned behaviour or the health belief theory (Askelson et al., 2010; Gerend and Shepherd, 2012).

\subsection{Conclusion}

In conclusion, about $77 \%$ of the suspected rabid dog bite victims visited a health centre while $57 \%$ receive sufficient doses of PEP. The important factors that influence victim's medical treatment seeking behaviour were found to be ownership status of the biting dog, the severity of the bite, body part bitten, monthly spending and distance to the nearest health centre delivering PEP whereas the likelihood of receiving sufficient doses of PEP were determined by monthly spending and distance to health centre. However, these factors did not explain all the variation among the three districts. The district in which victims lived appeared to have a relevant influence on the likelihood of seeking medical treatment but did not improve the prediciton on the likelihood of treatment compliance. Given the insights obtained from this study, policy directions that favor increased accessibility of health centres delivering PEP services could improve health seeking behaviour. In urban and rural highland districts, around four fifths of exposed persons who seek medical treatment comply with treatment regimen, indicating that the promotion of medical treatment tends to be beneficial. The district in which victims lived appeared to have importance in terms of the likelihood that they would seek medical treatment but was of less importance when attempting to predicit the likelihood of treatment compliance. 


\section{Ethics approval and consent to participate}

The study protocol was approved by the scientific and ethical review committee of Ethiopian Public Health Institute (Reference No. EPHI-6-13-824). Oral consent was obtained from participants prior to data collection.

\subsection{References}

Askelson, N. M., Campo, S., Lowe, J. B., Smith, S., Dennis, L. K., Andsager, J. 2010. Using the Theory of Planned Behavior to Predict Mothers’ Intentions to Vaccinate Their Daughters Against HPV. The Journal of School Nursing, 26(3), 194-202. doi:10.1177/1059840510366022.

Ayele, W., Fekadu, M., Zewdie, B., Beyene, M., Bogale, Y., Mocha, K., Egziabher, F. 2001. Immunogenicity and efficacy of Fermi-type nerve tissue rabies vaccine in mice and in humans undergoing post-exposure prophylaxis for rabies in Ethiopia. Ethiopian Medical Journal, 39(4), 313-321.

Beyene, T. J., Kidane, A. H., Mourits, M. C., Hogeveen, H. 2017. Public health burden and exposure costs of rabies in Ethiopia. SVEPM conference, March 29-31, 2017. Inverness, Scotland, UK.

Bichmann, W., Diesfeld, H.-J., Agboton, Y., Gbaguidi, E. A., SimshÄUser, U. 1991. District health systems: users' preferences for services in Benin. Health policy and planning, 6(4), 361-370.

Bishwajit, G., Ide, S., \& Ghosh, S. (2014). Social Determinants of Infectious Diseases in South Asia. International Scholarly Research Notices, 2014, 10. doi:10.1155/2014/135243

Chibwana, A. I., Mathanga, D. P., Chinkhumba, J., Campbell, C. H. 2009. Socio-cultural predictors of health-seeking behaviour for febrile under-five children in MwanzaNeno district, Malawi. Malaria Journal, 8(1), 219.

Cleaveland, S., Fevre, E. M., Kaare, M., Coleman, P. G. 2002. Estimating human rabies mortality in the United Republic of Tanzania from dog bite injuries. Bulletin of the World Health Organization, 80(4), 304-310.

CSA. 2013. Population Projection of Ethiopia for All Regions At Wereda Level from 2014 2017. Federal Democratic Republic of Ethiopia Central Statistical Agency,, Addis Ababa, Ethiopia.

Deressa, A., Ali, A., Bayene, M., Selassie, B. N., Yimer, E., Hussen, K. 2010. The status of rabies in Ethiopia: A retrospective record review. Ethiopian Journal of Health Development, 24(2).

Desta, S and Coppock, DL.2004. Pastoralism under pressure: Tracking system change in southern Ethiopia. Human Ecology 32(4): 465-486. 
Digafe, R. T., Kifelew, L. G., Mechesso, A. F. 2015. Knowledge, attitudes and practices towards rabies: questionnaire survey in rural household heads of Gondar Zuria District, Ethiopia. BMC Res Notes, 8(1), 400.

Dohoo, I. R., Martin, W., Stryhn, H. E. 2003. Veterinary epidemiologic research.

EHNRI. 2012. Proceding of the national workshop on Rabies prevention and control in Ethiopia, Oct 18-19, 2012, Addis ababa.

Feyisetan, B. J., Asa, S., Ebigbola, J. A. 1997. Mothers' management of childhood diseases in Yorubaland: the influence of cultural beliefs. Health Transition Review, 7(2), 221-234.

Franka, R., Smith, T. G., Dyer, J. L., Wu, X., Niezgoda, M., \& Rupprecht, C. E. 2013. Current and future tools for global canine rabies elimination. Antiviral Research, 100(1), 220-225. doi:http://dx.doi.org/10.1016/j.antiviral.2013.07.004.

Frew, S. E., Liu, V. Y., Singer, P. A. 2009. A business plan to help the 'global South'in its fight against neglected diseases. Health Affairs, 28(6), 1760-1773.

Gerend, M. A., Shepherd, J. E. 2012. Predicting human papillomavirus vaccine uptake in young adult women: Comparing the Health Belief Model and Theory of Planned Behavior. Annals of behavioral medicine: a publication of the Society of Behavioral Medicine, 44(2), 171-180. doi:10.1007/s12160-012-9366-5.

G/hiwot, T. T., Sime, A. G., Deresa, B., Tafese, W., Hajito, K. W., Gemeda, D. H. 2016. Community Health Seeking Behaviour for Suspected Human and Animal Rabies Cases, Gomma District, Southwest Ethiopia. Plos One, 11(3), e0149363. doi:10.1371/journal.pone.0149363

Garg, S. R. 2014. Rabies in Man and Animals: Springer.

Hampson, K., Coudeville, L., Lembo, T., Sambo, M., Kieffer, A., Attlan, M., . . Cleaveland, S. 2015. Estimating the Global Burden of Endemic Canine Rabies.

Hampson, K., Dushoff, J., Cleaveland, S., Haydon, D. T., Kaare, M., Packer, C., Dobson, A. 2009. Transmission dynamics and prospects for the elimination of canine rabies. Plos Biology, 7(3), e1000053.

Hemachudha, T., Laothamatas, J., Rupprecht, C. E. 2002. Human rabies: a disease of complex neuropathogenetic mechanisms and diagnostic challenges. The Lancet Neurology, 1(2), 101-109.

Hemachudha, T., Ugolini, G., Wacharapluesadee, S., Sungkarat, W., Shuangshoti, S., Laothamatas, J. 2013. Human rabies: neuropathogenesis, diagnosis, and management. The Lancet Neurology, 12(5), 498-513.

Hotez, P. J., Molyneux, D. H., Fenwick, A., Kumaresan, J., Sachs, S. E., Sachs, J. D., Savioli, L. 2007. Control of neglected tropical diseases. New England Journal of Medicine, 357(10), 1018-1027.

Jemberu, W. T., Molla, W., Almaw, G., Alemu, S. 2013. Incidence of rabies in humans and domestic animals and people's awareness in North Gondar Zone, Ethiopia. 
Kabeta, T., Deresa, B., Tigre, W., Ward, M. P., Mor, S. M. 2015. Knowledge, Attitudes and Practices of Animal Bite Victims Attending an Anti-rabies Health Centre in Jimma Town, Ethiopia. PLoS Negl Trop Dis, 9(6), e0003867.

Knobel, D. L., Cleaveland, S., Coleman, P. G., Fèvre, E. M., Meltzer, M. I., Miranda, M. E. G., . . . Meslin, F.-X. 2005. Re-evaluating the burden of rabies in Africa and Asia. Bulletin of the World Health Organization, 83(5), 360-368.

Kroeger, A. 1983. Anthropological and socio-medical health care research in developing countries. Social Science \& Medicine, 17(3), 147-161. doi:http://dx.doi.org/10.1016/0277-9536(83)90248-4.

Mackian, S., Bedri, N., Lovel, H. 2004. Up the garden path and over the edge: where might health-seeking behaviour take us? Health policy and planning, 19(3), 137-146. doi:10.1093/heapol/czh017

McFadden, D. 1978. Quantitative Methods for Analysing Travel Behaviour of Individuals: Some Recent Developments',(in) Hensher. DA and Stopher, PR (eds) Behavioural Travel Modelling, Croom Helm, London.

Mwangome, M., Prentice, A., Plugge, E., Nweneka, C. 2010. Determinants of appropriate child health and nutrition practices among women in rural Gambia. Journal of Health, Population and Nutrition, 167-172.

Ott, R., Longnecker, M. 2008. An introduction to statistical methods and data analysis: Cengage Learning.

Peters, D. H., Garg, A., Bloom, G., Walker, D. G., Brieger, W. R., Rahman, M. H. 2008. Poverty and access to health care in developing countries. Ann N Y Acad Sci, 1136, 161-171. doi:10.1196/annals.1425.011

Piyasirisilp, S., Hemachudha, T., Griffin, D. E. 1999. B-cell responses to myelin basic protein and its epitopes in autoimmune encephalomyelitis induced by Semple rabies vaccine. Journal of neuroimmunology, 98(2), 96-104.

Ramos, J. M., Melendez, N., Reyes, F., Gudiso, G., Biru, D., Fano, G., . . Balcha, S. 2015. Epidemiology of animal bites and other potential rabies exposures and anti-rabies vaccine utilization in a rural area in Southern Ethiopia. Annals of agricultural and environmental medicine: AAEM, 22(1), 76-79.

Sambo, M., Cleaveland, S., Ferguson, H., Lembo, T., Simon, C., Urassa, H., Hampson, K. 2013. The Burden of Rabies in Tanzania and Its Impact on Local Communities. Plos Neglected Tropical Diseases, 7(11), e2510.

Shaikh, B. T., Hatcher, J. 2004. Health seeking behaviour and health service utilization in Pakistan: challenging the policy makers. Journal of Public Health, 27(1), 49-54.

Shim, E., Hampson, K., Cleaveland, S., Galvani, A. P. 2009. Evaluating the cost-effectiveness of rabies post-exposure prophylaxis: A case study in Tanzania. Vaccine, 27(51), 71677172. doi:10.1016/j.vaccine.2009.09.027 
Sugiyama, M., Ito, N. 2007. Control of rabies: epidemiology of rabies in Asia and development of new-generation vaccines for rabies. Comparative Immunology, Microbiology and Infectious Diseases, 30(5), 273-286.

Teklu, G. G., Hailu, T. G., Eshetu, G. R. 2017. High Incidence of Human Rabies Exposure in Northwestern Tigray, Ethiopia: A Four-Year Retrospective Study. Plos Neglected Tropical Diseases, 11(1), e0005271.

Tepsumethanon, V., Wilde, H., Meslin, F. X. 2005. Six criteria for rabies diagnosis in living dogs. J Med Assoc Thai, 88(3), 419-422.

Tipping, G., Segall, M. 1995. Health Care Seeking Behaviour in Developing Countries: An Annotated Bibliography and Literature Review: Health Unit, Institute of Development Studies at the University of Sussex.

Tolossa, K., Debela, E., Athanasiadou, S., Tolera, A., Ganga, G., Houdijk, J. G. 2013. Ethnomedicinal study of plants used for treatment of human and livestock ailments by traditional healers in South Omo, Southern Ethiopia. Journal of Ethnobiology and Ethnomedicine, 9(1), 32.

Tschopp, R., Bekele, S., Aseffa, A. 2016. Dog Demography, Animal Bite Management and Rabies Knowledge-Attitude and Practices in the Awash Basin, Eastern Ethiopia. PLoS Negl Trop Dis, 10(2), e0004471.

Uzochukwu, B. S., Onwujekwe, O. E. 2004. Socio-economic differences and health seeking behaviour for the diagnosis and treatment of malaria: a case study of four local government areas operating the Bamako initiative programme in south-east Nigeria. International Journal for Equity in Health, 3(1), 6. doi:10.1186/1475-9276-3-6

WHO. 1984. WHO Expert Committee on Rabies. WHO Technical report series 709, Geneve, 1984.

WHO. (2005). WHO technical report series 931: WHO expert consultation on rabies; first report. Geneva Switzerland: WHO, p13.

WHO. (2013). WHO Expert Consultation on Rabies. Second report. World Health Organization technical report series(982), 1.

WHO. (2016). (World Health Organization) http://www.who.int/mediacentre/factsheets/fs099/en/ accesed date 15/4/2016.

Yibrah, M., \& Damtie, D. (2015). Incidence of human rabies exposure and associated factors at the Gondar Health Centre, Ethiopia: a three-year retrospective study. Infectious diseases of poverty, 4(1), 3. doi:10.1186/2049-9957-4-3 

Chapter 7

\section{Evaluation of canine vaccination strategies to control rabies in Ethiopia}

Tariku Jibat Beyene, Meagan C Fitzpatrick, Alison P Galvani, Monique CM Mourits, Henk Hogeveen

Under preparation to be submitted to The Lancet Global Health journal 


\begin{abstract}
In Ethiopia, rabies leads to approximately 3,000 human deaths every year. The economic burden is also high: one in five livestock-raising households lose cattle due to an attack from a rabid dog, jeopardizing livelihoods and food security for affected households. Currently, canine rabies vaccination coverage is only $18 \%$ in urban areas and non-existent in rural areas, contributing to an incidence of human rabies that is more than three times higher in the latter. The objective of this study was to evaluate the cost-effectiveness of canine vaccination in two Ethiopian districts, urban and rural. We built a dynamic epidemiological model, fit to districtspecific data on human rabies exposures and canine demography using a Bayesian Monte-Carlo Markov chain (MCMC) process. Epidemiological model output was coupled with an economic analysis to predict the human health impact (in disability-adjusted life-years (DALY)) and economic benefit (reducing human treatment costs and livestock rabies-related losses) across a range of vaccination scenarios. Human exposures, human deaths, and rabies-related livestock losses decreased monotonically with increasing vaccination coverage. In the rural district, all vaccination scenarios were found to be cost-saving compared to the current situation (status quo) of no vaccination. In both urban and rural districts, $50 \%$ coverage was identified as most likely scenario to provide the greatest net health benefits at the WHO-recommended willingness-to-pay threshold over a time frame of 10 years. A shorter analytical time frame leads to recommendations for higher coverage in both districts, as does even a slight threat of rabies reintroduction. This study showed a different cost-effective canine vaccination coverage between urban and rural district under strict consideration the benefit from human health alone In rural Ethiopia, the burden of rabies would not be complete without incorporating the livestock rabies, highlighting the importance of applying a broad perspective with regard to the benefit of vaccination. Such type of analysis including cattle and other rabies susceptible livestock species and the recommendation made are likely to apply for many rural communities in Africa maximizing the benefits of canine vaccination.
\end{abstract}




\subsection{Introduction}

Rabies claims the lives of 24,000 people in Africa annually (Yousaf et al., 2012), but efforts to control the disease are still lacking, particularly in sub-Saharan African nations such as Ethiopia. Rural communities are especially impacted by rabies outbreaks, through the combination of limited access to healthcare and the threat to their livelihoods through rabies attacks on livestock (Jibat et al., 2016; Hampson et al., 2015).

In Ethiopia, approximately 97,000 people are exposed to rabies annually, resulting in \$2 million spent for post-exposure treatment and approximately 3,000 deaths (Beyene et al., 2017). In addition, Ethiopia incurs one of the highest burdens of livestock rabies in the world, with annual national economic loss from cattle rabies estimated between \$209 million (Jibat et al., 2016) and \$412 million (Hampson et al., 2015).

While post-exposure prophylaxis effectively prevents human deaths following a rabies exposure, it can be expensive for those in resource-limited settings, and it has little impact on the primary source of the disease (Lavan et al., 2017). As more than 99\% of all human cases worldwide result from the bite of a domestic dog (WHO, 2005 ) the World Health Organization (WHO) recommends canine rabies vaccination to eliminate the disease in canine populations and consequently from humans. However, canine vaccination coverage in Ethiopia remains one of the lowest in the world (Deressa et al., 2010; Hampson et al., 2015; Jibat et al., 2016). Canine vaccination has never been enforced as compulsory nor promoted as a public good, thus very few dog owners vaccinate their dogs in Ethiopia (Ali et al., 2010; Kabeta et al., 2015).

Cost-effectiveness analyses are relevant when there are competing public health interests, especially in resource-limited situations (Lembo et al., 2010). Cost-effectiveness studies conducted for canine vaccination programs in Africa have found $70 \%$ coverage to be optimal (recommended) to eliminate rabies (Zinsstag et al., 2009; Fitzpatrick et al., 2014). However, African communities can differ from each other with regard to human settlement, dog density, and other features relevant to the success and efficiency of vaccination campaigns. Even within Ethiopia, variation between urban and rural agro-ecologies with regard to human and canine densities and in pet-owning practices (Gsell et al.,2012) could lead to differences in the epidemiological benefits and cost-effectiveness of vaccination. Therefore, setting-specific evaluation is imperative.

Previous studies focused on the direct impacts with respect to human health and not its indirect effects on livelihoods by affecting livestock. The additional economic benefit from rabies control in livestock could potentially justify the additional costs of vaccination campaigns with higher coverages than would otherwise be efficient from a strict human health perspective. Therefore, to evaluate the optimal (recommended and cost-effective) program for rabies control 
in Ethiopia, there is a need for an overarching One-Health oriented cost-effectiveness analysis that includes the benefits of eliminating rabies in the domestic dog population, preventing human rabies cases, and minimizing rabies-related livestock losses in cattle-keeping communities. In this study, we evaluate the epidemiological benefits and cost-effectiveness of different canine vaccination strategies in two districts, urban and rural, of Ethiopia.

\subsection{Materials and Methods}

We adopted a canine rabies transmission model previously developed by Fitzpatrick and colleagues (Fitzpatrick et al., 2014) fitted to district-specific retrospective case finding data on human rabies exposures and deaths. We integrated this epidemiologic model with an economic model of human and cattle rabies related and canine vaccination campaign costs. The combined model was simulated over 10 years of canine vaccination campaigns varying from status quo (i.e. current vaccination coverage) to $90 \%$ coverage, quantifying the impact of each coverage on human exposure, health burden (DALY), health costs, cattle related economic loses and campaign costs. The district-specific optimal strategy, i.e a strategy with the highest net health benefit at a given a willingness-to-pay threshold, was chosen based on cost-effectiveness thresholds recommended by WHO (WHO, 2010).

We compared two Ethiopian districts, Bishoftu and Lemuna-bilbilo, representative of two different degrees of urbanization in Ethiopia. Bishoftu is an urban district with a human density of 3500 inhabitants $/ \mathrm{km}^{2}$ (CSA, 2013), an average canine density of $340 \mathrm{dogs} / \mathrm{km}^{2}$, and a human rabies exposure rate of 135 rabid dog bites per 100,000 inhabitants (Beyene et al., 2017). Lemuna-bilbilo is a rural district, substantially less dense that Bishoftu at 158 inhabitants $/ \mathrm{km}^{2}$ (CSA, 2013), an average dog density of $35.4 \mathrm{dogs} / \mathrm{km}^{2}$ and a human rabies exposure rate of 101 per 100,000 inhabitants (Beyene et al., 2017).

\subsubsection{Epidemiological model}

\section{Model structure}

We constructed a stochastic compartmental rabies transmission model for domestic dogs (Figure 7.1). The canine population $(\mathrm{N})$ is divided among five classes: susceptible (S), exposed $(\mathrm{E})$, infectious (I), vaccinated (V) and dead (R). Dogs are born into the vaccinated class at rate $b v(S+V+E)$, and into the susceptible class at rate $b(1-v)(S+V+E)$. Infectious and exposed dogs were excluded from the reproductive rate, as the virus typically has a short incubation period and puppies born to rabid animals are unlikely to survive (Fitzpatrick et al., 2014). Upon exposure to rabies at rate $\beta \mathrm{SI} / \mathrm{K}$, dogs move to the exposed class and then transition to the infectious class at rate $\sigma, \beta$ being dog-to-dog transmission rate. Death from rabies occurs at rate $\alpha$. Death from all other causes, excluding rabies but including carrying capacity and resource constraints, removes animals from all compartments, proportional to compartment size, at a density-dependent death rate $\gamma$ that increases as the population size approaches its carrying 
capacity, $K$, according to the Verhulst logistic equation (Bacar, 2011).

Humans and cattle are dead-end hosts and are exposed to rabid dogs at rates $\varepsilon$ and $\omega$, respectively. Following human exposure, a human receives sufficient doses of prophylaxis vaccine with probability $p$. We assume all recipients of vaccine survive, but that non-recipients have a probability $m$ of death, which itself relies on the distribution of bite wounds across the body and the probability of death associated with exposure to each wound type. For this model, cattle exposure is equivalent to cattle loss, as we calculate $\omega$ from observed cattle loss with no intermediate events.

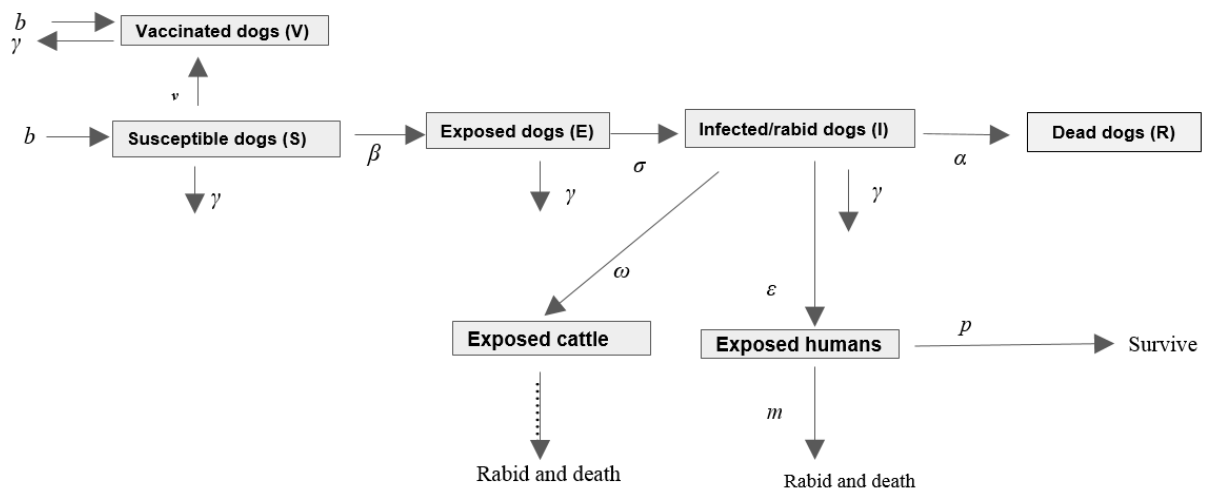

Figure 7:1: Rabies transmission model schematic.

( $b=$ birth rate; $\gamma=$ death rate; $\beta=$ dog-to-dog transmission rate; $\sigma=$ transition rate to the infectious class, $\alpha=$ rate of death from rabies; $\varepsilon=$ human exposure rate to rabid dogs; $\omega=$ cattle exposure rate to rabies; $p=$ probability of exposed human receives sufficient doses of the post-exposure prophylaxis (PEP); $v=$ proportion of susceptible dogs vaccinated, $m=$ probability of death after exposure and non-recipients of the PEP)

\section{Parameterization and fitting}

We used a Bayesian Monte-Carlo Markov chain (MCMC) process to fit the epidemiological and demographic parameters to district-specific empirical data on human rabies incidence and canine population size. Flat priors were specified for $\beta$, the dog-to-dog rabies transmission rate, and $K$, the canine population carrying capacity. For all other parameters, prior distributions were defined with empirical data, either collected specifically for this study or published in the literature (see Supplementary information 7.1: Table 1). As several parameters differ between districts, fitting was conducted separately for each district.

The per capita birth rate $(b)$ was assumed to be the product of the sex ratio $(h)$, the average litter size (l) and litter frequency ( $f$ ), and pup survival (s) (Hampson et al., 2009). A district-specific 
sex ratio was estimated from a field survey conducted in both districts in April and May 2015 (see Supplementary information 7.1: Dog demographics). Canine density was estimated from the same survey which used techniques of capture-mark and re sight (Hiby et al., 2011). Assuming similarities between the agro-ecologies, we derived estimates of litter size, litter frequency, and pup survival from studies in Tanzania (Hampson et al., 2009). Prior distributions for the rabies incubation period, $1 / \sigma$, and the infectious period, $1 / \alpha$, and the dog-to-human bite rate, $\delta$, were also derived from Tanzania (Hampson et al., 2009).

District-specific human rabies exposures and death cases were collected from health centers in both districts from Sept 2013 through August of 2014 (Beyene et al., 2017). Contact tracing further identified victims who did not report to health centers through 'snowballing" exercise. Exposures and deaths from potentially rabid dogs were identified using six retrospective diagnostic criteria for the biting dogs (Tepsumethanon et al., 2005). In Bishoftu, 189 likely human exposures and 1 death, and in Lemuna-Bilbilo, 189 likely human exposures and 8 deaths were identified to be from potentially rabid dogs (see Supplementary information 7.1: Human rabies exposure data collection and contact tracing). With this survey data, we set the prior distribution for the post-exposure treatment probability $p$ as Beta $(147,42)$ for Bishoftu and Beta $(95,94)$ for Lemuna-Bilbilo. We drew from a multinomial distribution characterizing wound location, and assumed Beta distributions for wound-specific probabilities of developing rabies and then death from our previous study (Beyene et al., 2017) and Tanzania (Shim et al., 2009) (see Supplementary information 7.1: Table 1).

We constructed a likelihood function to evaluate the probability of the data on human exposure, human death, and canine population size, using Poisson distributions around the human exposure and death data and a normal distribution around canine population size. For each district:

$$
\mathrm{L}(x, d, n, q \mid \lambda, \mu, \varphi)=\frac{\lambda^{x} e^{-\lambda}}{x !} * \frac{\mu^{d} e^{-\mu}}{d !} * \frac{1}{q \sqrt{2 \pi}} e^{\frac{(\varphi-n)^{2}}{2 q^{2}}}
$$

Where $x$ is the number of human rabies exposures, $d$ is the number of human deaths, $n$ and $q$ are the mean and standard error for canine density. The parameters $\lambda, \mu$, and $\varphi$ represent the model estimates for annual human rabies exposures, annual human rabies deaths, and canine density respectively.

The differential equations underlying the SEIVR model were solved in continuous time in $\mathrm{R}$ statistical program (R Core Team, 2014) using the package “deSolve” (Soetaert et al., 2010). For each district, we used the Metropolis-Hasting algorithm (Carlo, 2004) to generate four chains of 20,000 iterations each, initialized at dispersed values for $\beta$ and $K$. We applied the 
Gelman-Rubin diagnostic (Brooks and Gelman, 1998; Gelman and Rubin, 1992) in the R package “coda” (Plummer et al., 2008) to test for convergence. We thinned each chain by a factor of 25 and combined the final 250 parameter sets from each thinned chain to create each district-specific joint posterior distribution. The parameter set in the joint distribution with the highest likelihood was designated as the representative set, and used for base-case analyses.

\section{Interventions}

For each of the 1000 parameter sets in the joint posterior distribution, and for each district separately, we simulated annual rabies mass vaccination campaigns, at coverage varying from status quo (i.e. 18 in Bishoftu/Urban and no vaccination in Lemuna-bilbilo/rural districts) to $90 \%$ in increments of $10 \%$. Annual canine vaccination campaigns were assumed to be delivered once a year under an intensive campaign executed within a few days or weeks per district. We also assumed previously vaccinated dogs would be revaccinated, and no vaccine waning from an initial efficacy of $100 \%$ would occur within the year.

\subsubsection{Economic model}

We evaluated the total costs of rabies under each strategy consisting of the costs associated with the vaccination campaign, human post-exposure treatment (PET), and the rabies-related cattle losses.

\section{Vaccination campaign costs}

The costs of canine vaccination were based on a previously standardized economic model (Wera et al., 2013). Accordingly, the costs of mass canine vaccination include the costs of the vaccine, consumables (needle and syringe, ice bar, disinfectant and swab, certificate and collar), temporary vaccinators and supervisors (per-diem, training, and transportation), advertising, and capital costs (refrigerator, muzzle, and cool bags). We assumed that a short-acting canine rabies vaccine currently produced by the National Veterinary Institute in Ethiopia would be purchased at its currently proposed price and would provide at least one year of full protection. Other costs of vaccination campaign were established through a market survey. We incorporated increasing dog search costs for coverage rising above $50 \%$ in both districts. We also incorporated additional transportation costs at low coverage below 50\% in Lemuna-bilbilo, as teams must travel longer distances between villages/dogs to ensure an even geographic distribution of vaccinated dogs. Detailed procedures of costs estimation for dog vaccination campaigns and inputs are listed in Supplementary information 7.1: Table 2.

\section{Human health and economic burden}

We quantified the human rabies burden in terms of both the costs of treating rabies-exposed people with PET as well as the disability-adjusted life years (DALY) incurred from rabies mortality. PET costs consist of health care costs for wound care, tetanus anti-toxin and post- 
exposure prophylaxis vaccine, as well as the non-healthcare costs of lost productivity for the victim and any accompanying family associated with seeking care (WHO, 2014). Our previous study (Beyene et al., 2017) estimated that the average PET costs for individuals who completed the regimen recommended by Ethiopian Public Health Institute (EHNRI, 2012) were \$23.2 and \$30.4 in urban and rural districts, respectively.

To estimate health burden, we considered only years of life lost (YLL) by individuals who developed rabies. We did not consider morbidity (temporary disability) as the disease is acute and swiftly fatal (Hotez et al., 2014). We estimated the YLL per human rabies case from the primary data on age from rabies deaths in each district, assuming a multinomial distribution across 5-year age intervals. The average years of life lost incurred per human rabies death was calculated by multiplying the number of deaths within an age category multiplied by the life expectancy of the age group (i). The average life expectancy for Ethiopia is derived from the life table estimate of 2013 as published by WHO (WHO, 2013a).

We calculated the PET costs per rabid dog as the product of the rate at which a rabid dog bites a human $(\delta)$, the post-exposure treatment probability $(p)$, and PET costs. Similarly, the health burden per rabid dog was estimated by multiplying the probability of a rabid dog biting a human $(\delta)$, a probability that the victim will not receive sufficient treatment (1-p) and the life-years lost per human rabies death (see Supplementary information 7.1: Table 3). To estimate the total health and economic costs of rabies under each canine vaccination strategy, we multiplied these per-rabid-dog losses by the number of rabid dogs in the epidemiological model predictions.

\section{Costs of cattle rabies}

In rural Ethiopia, the economic impact of rabies is largely mediated by cattle exposure. Nearly all cattle rabies exposures are from rabid dogs and canine vaccination is currently non-existent (Okell, et al., 2013). Because cattle are dead-end hosts and not active members of the rabies transmission cycle, we assumed that cattle rabies is reduced commensurately with dog rabies through a canine vaccination campaign. Specifically, the cattle rabies-related economic loss under status quo $(L s q)$ equals the product of herd-level rabies incidence (Hinc) under status quo, the number of herds in the district (Nherd), and the economic loss per affected herd (Lpah). Under a specific canine vaccination strategy, this product is modified by the proportion of dogs which are rabid compared to the status quo ( rabid $\propto$ ). Economic parameters and status quo cattle rabies incidence were extracted from our previous study on economic impact of cattle rabies as described in supplementary information (see Supplementary information 7.1: Table 4).

\subsubsection{Cost-effectiveness evaluation}

We conducted a cost-effectiveness evaluation to identify the most efficient strategies for rabies 
control in Ethiopia. We assessed each district separately, tailoring the analysis to urban and rural settings. All base case analyses were conducted on a time frame of 10 years with a $3 \%$ discount rate, as recommended by the World Bank Disease Control Priorities Study and the Global Burden of Disease project (Mathers et al., 2001).

With the base-case predictions for each district, we first identified the non-dominated vaccination strategies, or those for which greater health benefits could not be achieved at a lower costs per life-year. Then, we calculated the incremental cost-effectiveness ratio (ICER) of moving from one specific canine vaccination coverage to the next more-costly nondominated coverage (Jha et al., 2006). Those strategies with a cost-per-DALY falling at or below Ethiopia's per-capita GDP of 568\$ (World Bank, 2015), or three times this value (i.e. \$1704), are considered “very cost-effective” or “cost-effective,” respectively (WHO, 2010).

To incorporate parameter uncertainty into our identification of the optimal rabies control strategies, we also applied a net health benefit (NHB) framework (Briggs et al., 2002). Net benefits are calculated for a given strategy as the difference between the health benefit (in DALY) provided by that strategy compared to status quo and its associated campaign costs, divided by the societal willingness-to-pay for DALYs. This framework gives a single outcome measure that identifies the strategy which provides the largest overall benefit at that willingnessto-pay. For each of 1000 parameter sets drawn, we identified the strategy that conferred the most net benefits at a value of willingness-to-pay three times the GDP per capita. We then tabulated the probability that each strategy would confer the most net benefits, and the strategy with the highest probability was considered “optimal” at that threshold (Barton et al., 2008).We conducted this analysis across societal willingness-to-pay thresholds ranging from \$1 to \$5000 per DALY saved.

\section{Sensitivity analyses}

A series of one-way sensitivity analyses were performed using the net benefits framework, assessing the sensitivity of the optimal strategy choice to changes in dog rabies vaccine price, number of dogs vaccinated daily per vaccinator team, training and advertising costs during vaccination campaign, PET costs, and cattle rabies herd level incidence. For each, the specific input parameter was altered at its alternative point estimate, maintaining the base case for all other parameters. Two-way sensitivity analyses were similarly performed on the time frame and discount rate.

For the rabies transmission parameters, we also performed sensitivity analyses around the bite rate and rabies reintroduction through rabid dogs from neighbouring villages. We assumed a lower (0.2) and a higher (0.99) rabid dog-human bite rate in case more rabid dogs are killed or not killed and consequently having lower or higher chance to bite humans, respectively. 
Considering the possibility of rabies reintroduction from neighbouring villages, we assumed an equivalent of $2 \%$ and $4 \%$ of the rabid dogs are reintroduced to the districts every year. For each scenario of re-introduction, the MCMC process was re-fitted and run with the new parameters and the new optimal coverage around the Incremental cost-effectiveness ratios were estimated.

\subsection{Results}

\subsubsection{Estimated model parameters}

Estimation of epidemiological parameters and model validation

Fitting the rabies epidemiological model to the data, the most likely carrying capacity for dogs (K) was estimated as $356 \mathrm{dogs} / \mathrm{km}^{2}$ (95\% CI: 345-371) and $43 \mathrm{dogs} / \mathrm{km}^{2}$ (95\% CI: 41.8-45.2), for Bishoftu and Lemuna-bilbilo districts respectively. The dog-to-dog transmission rates $(\beta)$ were estimated to be 0.39 (95\% CI: 0.39-0.47) and 0.40 (95\% CI: 0.36-0.44) daily transmission events per rabid dog, and dog-to-human bite rate $(\delta)$ estimated as 0.56 (95\% CI: 0.45-0.57) daily transmission events per rabid animal, both in Bishoftu and Lemuna-bilbilo districts. The incubation period $(1 / \sigma)$ was estimated as 22.5 (95 \% CI: 19.9-24.6) and 21.6 (95 \% CI: 19.824.8) days while the infectious period $(1 / \alpha)$ as 3.13 (95\% CI: 2.87-3.38) and 3.08 (95\% CI: 2.88-3.35), for Bishoftu and Lemuna-bilbilo respectively. The probability of a human developing rabies following exposure and without treatment was 0.16 (95\% CI: 0.15-0.17) in both districts. The post-exposure prophylaxis coverage was estimated to be 0.83 (95\% CI: 0.740.84 ) and 0.59 (95\% CI 0.45-0.59) for Bishoftu and Lemuna-bilbilo districts respectively (Table 7.1).

The joint posterior distribution produced estimates of human rabies exposure consistent with the data from each district. In Bishoftu, the model predicted 190 annual human exposures (95\% CI: 188-193) and 6 deaths (95\% CI: 4.5-8.0 deaths), compared with an observed 189 exposures and one death. In Lemuna-bilbilo the model predicted 184 annual human exposures (95\% CI:182-187) and 11.6 deaths (95\% CI: 11.4-11.7), compared with an observed 189 exposures, and 8 deaths. Under status quo coverage, the fitted epidemiological model estimated the annual number of rabid dogs to be 340 (95\% CI: 336-345) and 330 (95\% CI: 325-335) in Bishoftu and Lemuna-bilbilo districts respectively. 
Table 7.1 Posterior distribution of epidemiological parameters).

\begin{tabular}{llll}
\hline Parameters & Description & \multicolumn{2}{l}{ ML* estimates and 95\% CI } \\
\hline \multirow{2}{*}{$\beta$} & dog to dog transmission/day & $0.39(0.39-0.47)$ & $0.40(0.37-0.44)$ \\
$\mathrm{K}$ & Carrying capacity (dogs/km²) & $356(345-371)$ & $42.6(41.8-45.2)$ \\
$1 / \sigma$ & Incubation period (days) & $22.5(19.9-24.6)$ & $21.6(19.8-24.8)$ \\
$1 / \alpha$ & Infectious period (days) & $3.13(2.87-3.38)$ & $3.08(2.88-3.35)$ \\
$\delta$ & Rabid dog-human bite rate & $0.56(0.45-0.57)$ & $0.56(0.46-0.57)$ \\
$\mathrm{m}$ & Probability of developing rabies & $0.16(0.15-0.17)$ & $0.16(0.15-0.17)$ \\
$\mathrm{p}$ & Post-exposure prophylaxis & $0.83(0.74-0.84)$ & $0.59(0.45-0.59)$ \\
\hline
\end{tabular}

*ML= maximum likelihood

\section{Human rabies exposure predictions}

The predicted number of rabid dog cases and consequently human exposures decreased monotonically as vaccination coverage increased (see Supplementary information 7.1: Figure 1a and 1b). In Bishoftu, the mean annual number of rabid dogs declines to 0.18 (95\% CI: 0-1.4) at $90 \%$ coverage (see Supplementary information 7.1: Figure 1a). In Lemuna-bilbilo, the mean annual number of rabid dogs drops to 0.27 (95\% CI: 0-2.08) at $90 \%$ coverage (see Supplementary information 7.1: Figure 1b). In Bishoftu, all vaccination campaigns above $40 \%$ coverage lead to fewer than 1 rabid dog annually by the end of 4 years, while in Lemuna-bilbilo coverage above $20 \%$ leads to fewer than 1 rabid dogs annually by the end of 4 years. In both districts, high coverage achieves faster reductions in rabies than low coverage (see Supplementary information 7.1: Figure 7.1c-f). In urban Bishoftu, for the status quo (18\%) and $90 \%$ coverage, the cumulative number of human exposures over 10 years are 1907 and 0.98 (Figure 7.2c). Similarly, in the rural district, the cumulative number of human exposures over 10 years are 11840 and 0.67 under status quo (0\%) and 90\% coverage (Figure 7.2d).

\section{Human health burden in DALY lost}

As human deaths are the consequence of delay or failure to receive appropriate PET, these deaths were predicted to decrease proportionately with canine rabies incidence as canine vaccination coverage increases. Adjusting for district-specific data on the age of death, a single human death corresponded to 46.7 (95\% CI: 46.7-46.7) and 47.7 (95\% CI: 45.7-55.4) DALYs in Bishoftu and Lemuna-bilbilo, respectively. Adjusting further for PET access, each rabid dog is associated an estimated of 0.64 and 1.67 DALYs. The cumulative number of DALYs over 10 years under status quo and 90\% coverage are 2554.75 and 1.32 for Bishoftu and 7402.6 and 2.72 for Lemuna-bilbilo districts respectively. 


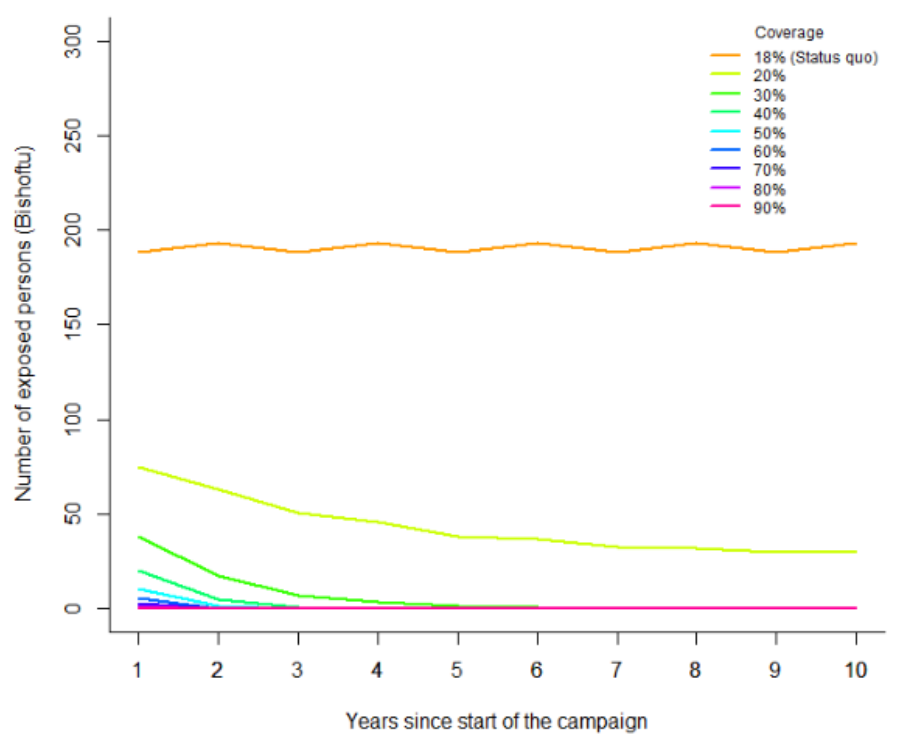

a)

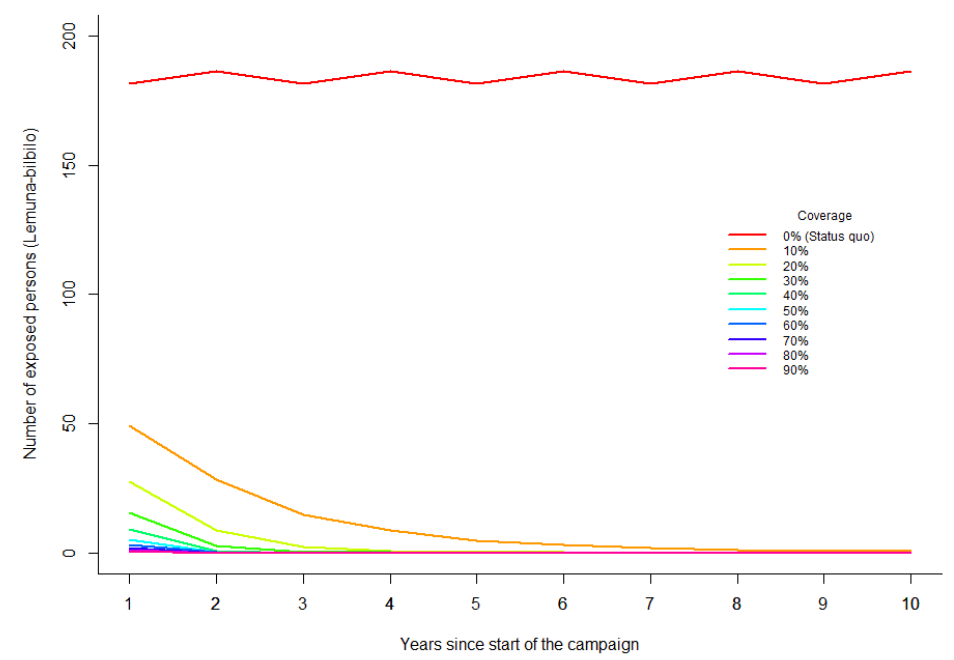

b) 


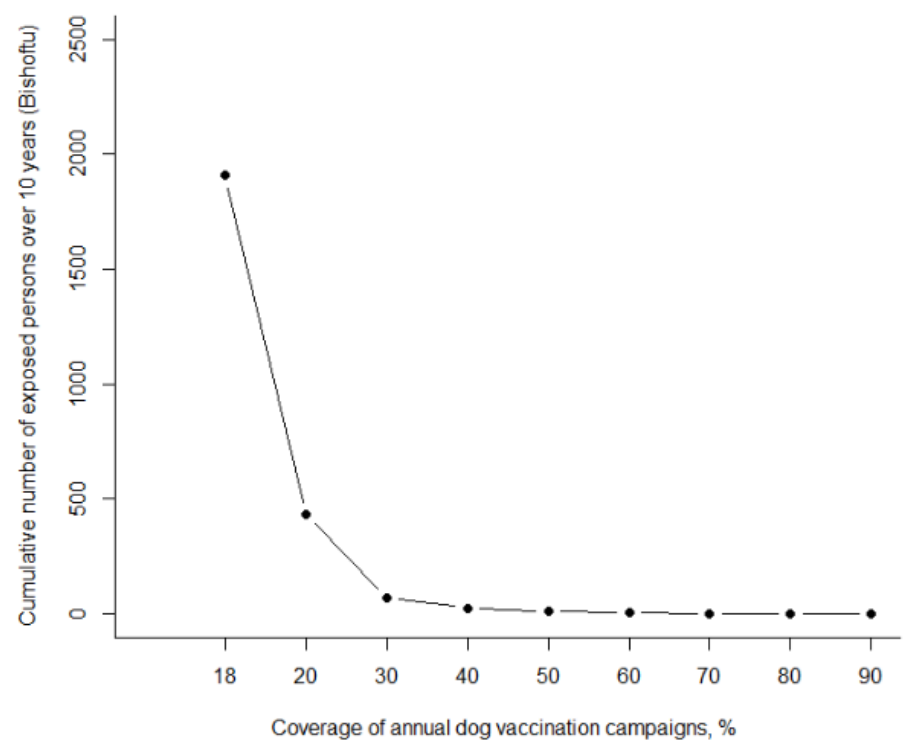

c)

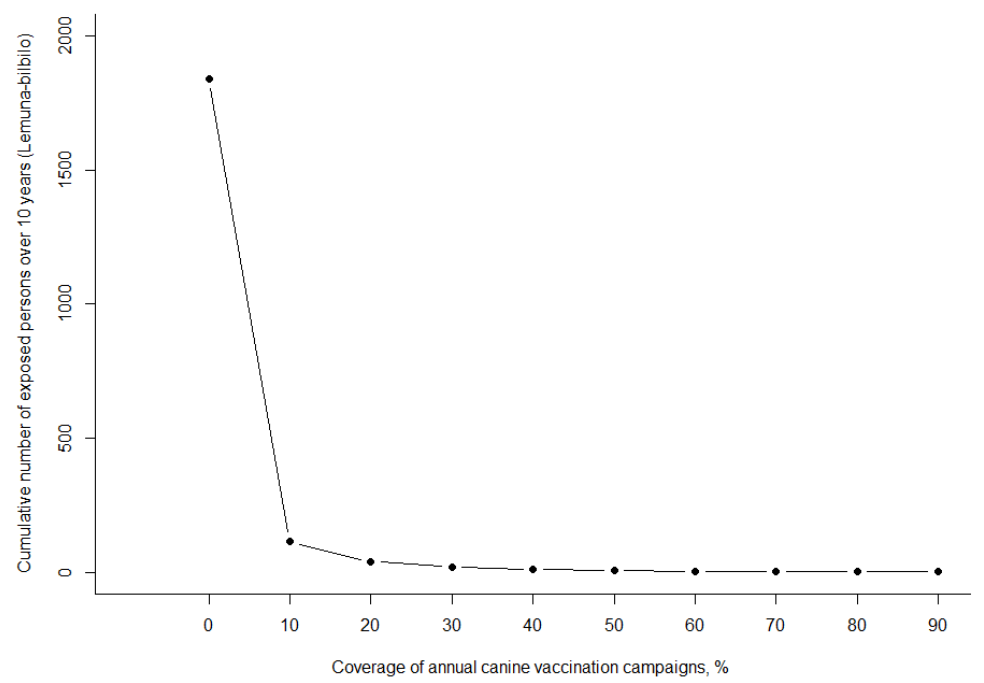

d)

Figure 7.2 Total number of human rabies exposures over 10 years for each vaccination coverage in a) Bishoftu and b) Lemuna-bilbilo, and cumulative number of human rabies exposures over 10 years in c) Bishoftu and d) Lemuna-bilbilo under different canine vaccination coverage strategies. 


\subsubsection{Economic burden of rabies and costs of intervention strategies}

Under the status quo, the mean annual costs for rabies control in Bishoftu (including canine vaccination and PET) were estimated at $\$ 9239\left(\$ 231 / \mathrm{km}^{2}\right)$. These mean annual costs reached a maximum of $\$ 20426\left(\$ 511 / \mathrm{km}^{2}\right)$ under 90\% coverage and a minimum of $\$ 7003\left(\$ 175 / \mathrm{km}^{2}\right)$ under $20 \%$ coverage. With the increase in vaccination coverage, the costs of PET decreased while the costs of dog vaccination increased. The total annual costs increased monotonically with coverages rising above $20 \%$, as vaccination campaign costs outstrip savings from reduced PET use (Figure 7.3a). All strategies, except for the vaccination coverage at $20 \%$,were more expensive than the status quo situation.

In Lemuna-bilbilo, besides the costs of providing PET , the economic losses due to cattle rabies declined with an increase in vaccination coverage as a result of a declined incidence in canine rabies (Figure 7.3b). The mean annual costs of rabies under the status quo (no vaccination) were estimated at $\$ 2,443,563\left(\$ 2063 / \mathrm{km}^{2}\right)$, consisting of $\$ 3323\left(\$ 2.8 / \mathrm{km}^{2}\right)$ PET costs, and $\$ 2,440,240\left(6854 / \mathrm{km}^{2}\right)$ of economic losses due to cattle rabies. The lowest costs strategy was shown to be at $50 \%$ coverage. All vaccination strategies had lower total costs compared to costs of the status quo situation with no vaccination. 


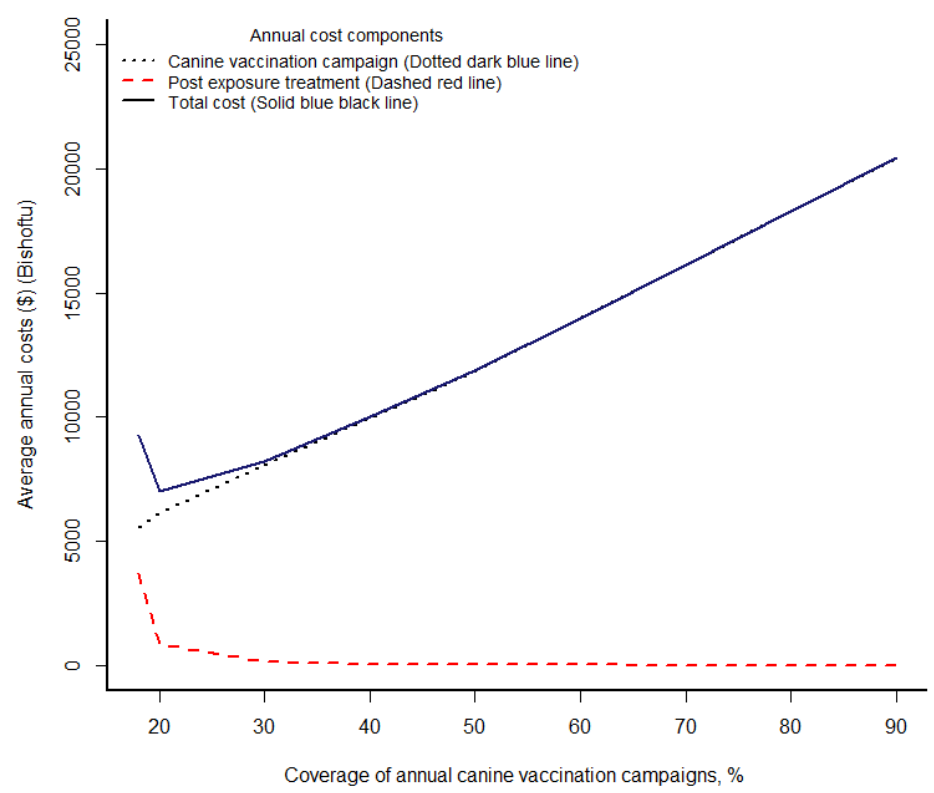

a)

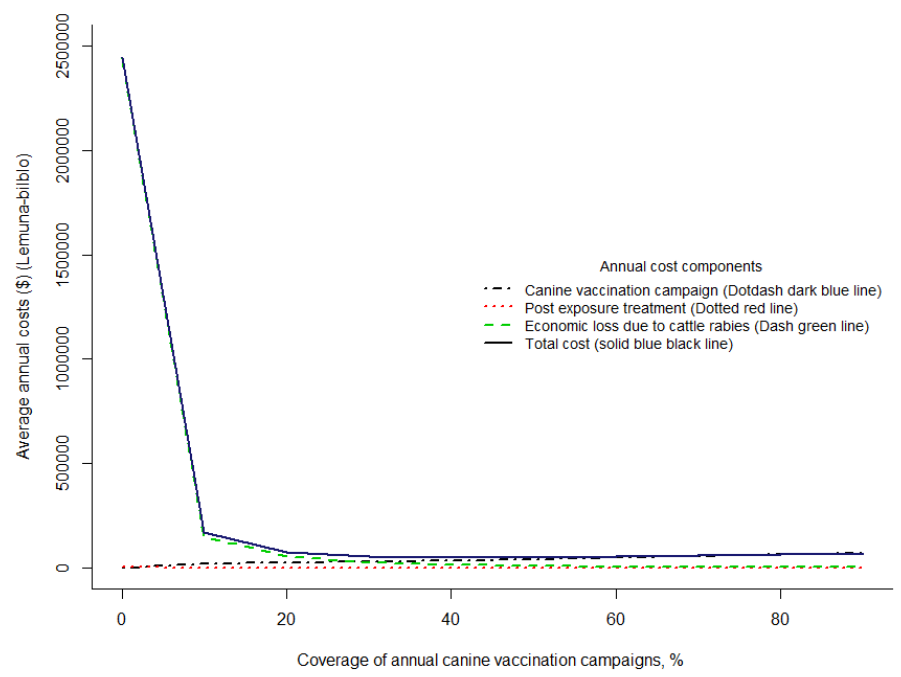

b)

Figure 7.3 Cost component and average annual (undiscounted) costs of rabies across different vaccination coverage a) Bishoftu and b) Lemuna-bilbilo. NB: The scales on the y axis are different as the average annual costs of rabies in Bishoftu has max of about 20,000 while Lemuna-bilbilo has max of 2,500,000. 


\subsubsection{Incremental cost-effectiveness ratios}

A canine vaccination coverage of $20 \%$ in Bishoftu and 50\% in Lemuna-bilbilo reduced more DALYs for less costs than campaigns at lower coverages. Accordingly, coverages less than the indicated coverage levels in the respective districts, are considered dominated. These coverage levels also represent the minimum cost strategy for each district. WHO-recommended thresholds for "very cost-effective" and "cost-effective" strategies in Ethiopia equal to \$568 and $\$ 1710$ per DALY saved, respectively. According to these thresholds, vaccination coverages of $30 \%$ and $40 \%$ are very cost-effective in Bishoftu, while a coverage of $50 \%$ reflects a costeffective strategy. In Lemuna-bilbilo, vaccination coverage of $50 \%$ is the only cost-effective strategy, as the ICER between $50 \%$ and $60 \%$ coverage exceeds the WHO-recommended threshold (Table 7.2).

Table 7.2. Cumulative costs over 10 years, and discounted to present value (3\% discount rate) in a) Bishoftu and b) Lemuna-bilbilo districts.

\begin{tabular}{|c|c|c|c|c|c|c|}
\hline \multicolumn{7}{|c|}{ a) Bishoftu/ urban district } \\
\hline $\begin{array}{l}\text { Vaccination } \\
\text { coverage (\%) }\end{array}$ & $\begin{array}{l}\text { Costs of } \\
\text { vaccination }\end{array}$ & $\begin{array}{l}\text { Costs of } \\
\text { PET }\end{array}$ & $\begin{array}{l}\text { Cattle rabies- } \\
\text { related loss }\end{array}$ & $\begin{array}{l}\text { DALY } \\
\text { s saved }\end{array}$ & $\begin{array}{l}\text { Total } \\
\text { economic } \\
\text { burden (\$) }\end{array}$ & $\begin{array}{l}\text { ICER } \\
\text { (\$/DALY) }\end{array}$ \\
\hline 18 & 48618 & 32557 & $N A$ & 0 & 81175 & Dominated \\
\hline 20 & 54096 & 7612 & $N A$ & 1466 & 61708 & Minimum cost \\
\hline 30 & 70840 & 1336 & $N A$ & 1835 & 72176 & 29 \\
\hline 40 & 87385 & 503 & $N A$ & 1884 & 87888 & 321 \\
\hline 50 & 103898 & 234 & $N A$ & 1900 & 104132 & 1027 \\
\hline 60 & 122757 & 119 & NA & 1905 & 122876 & 2770 \\
\hline 70 & 141612 & 63 & NA & 1910 & 141675 & 5752 \\
\hline 80 & 160464 & 35 & $N A$ & 1912 & 160499 & 11148 \\
\hline 90 & 179316 & 19 & NA & 1913 & 179335 & 20741 \\
\hline \multicolumn{7}{|c|}{ b) Lemuna-bilbilo/ rural district } \\
\hline $\begin{array}{l}\text { Vaccination } \\
\text { coverage (\%) }\end{array}$ & $\begin{array}{l}\text { Costs of } \\
\text { vaccination }\end{array}$ & $\begin{array}{l}\text { Costs of } \\
\text { PET }\end{array}$ & $\begin{array}{l}\text { Cattle rabies- } \\
\text { related loss }\end{array}$ & $\begin{array}{l}\text { DALY } \\
\text { saved }\end{array}$ & $\begin{array}{l}\text { Total } \\
\text { economic } \\
\text { burden (\$) }\end{array}$ & $\begin{array}{l}\text { ICER } \\
\text { (\$/DALY) }\end{array}$ \\
\hline $\begin{array}{r}\text { No } \\
\text { vaccination }\end{array}$ & 0 & 29493 & 21440219 & 0 & 21469712 & Dominated \\
\hline 10 & 152948 & 2238 & 1643717 & 3882 & 1798903 & Dominated \\
\hline 20 & 204058 & 824 & 608113 & 4083 & 812996 & Dominated \\
\hline 30 & 255180 & 403 & 297990 & 4143 & 553573 & Dominated \\
\hline 40 & 300446 & 220 & 163039 & 4169 & 463705 & Dominated \\
\hline 50 & 351082 & 127 & 94536 & 4182 & 445745 & Minimum cost \\
\hline 60 & 415283 & 77 & 56753 & 4190 & 472112 & 3631 \\
\hline 70 & 479483 & 47 & 34820 & 4194 & 514350 & 10030 \\
\hline 80 & 544885 & 29 & 21653 & 4196 & 566567 & 20667 \\
\hline 90 & 610359 & 18 & 13569 & 4198 & 623946 & 37002 \\
\hline
\end{tabular}




\section{Net Health Benefit uncertainty analysis}

Incorporating parameter uncertainty, we assessed the probability that each strategy would be optimal (i.e., provide the most net health benefits) across a range of willingness-to-pay values (Figure 7.4). We found that 50\% coverage was most likely to be optimal at the "cost-effective" threshold in both districts (Figure 7.4a and 7.4b). At the "very cost-effective" threshold, the optimal strategy would drop to $40 \%$ for Bishoftu but remain at $50 \%$ in Lemuna-bilbilo. We found zero probability that status quo would be optimal/recommended at all willingness-to-pay values in both districts. In both districts $50 \%$ vaccination coverage was the recommended/optimal choice but at a different probability of 0.71 and 0.91 in Bishoftu and Lemuna-bilbilo districts, respectively.

To allow direct comparison with Bishoftu, we analysed a scenario for Lemuna-bilbilo where we considered only human health and economic benefits, excluding the economic benefit of cattle rabies. We found that the recommended/optimal coverage would drop to $30 \%$ at costeffective threshold (Figure 7.4c).

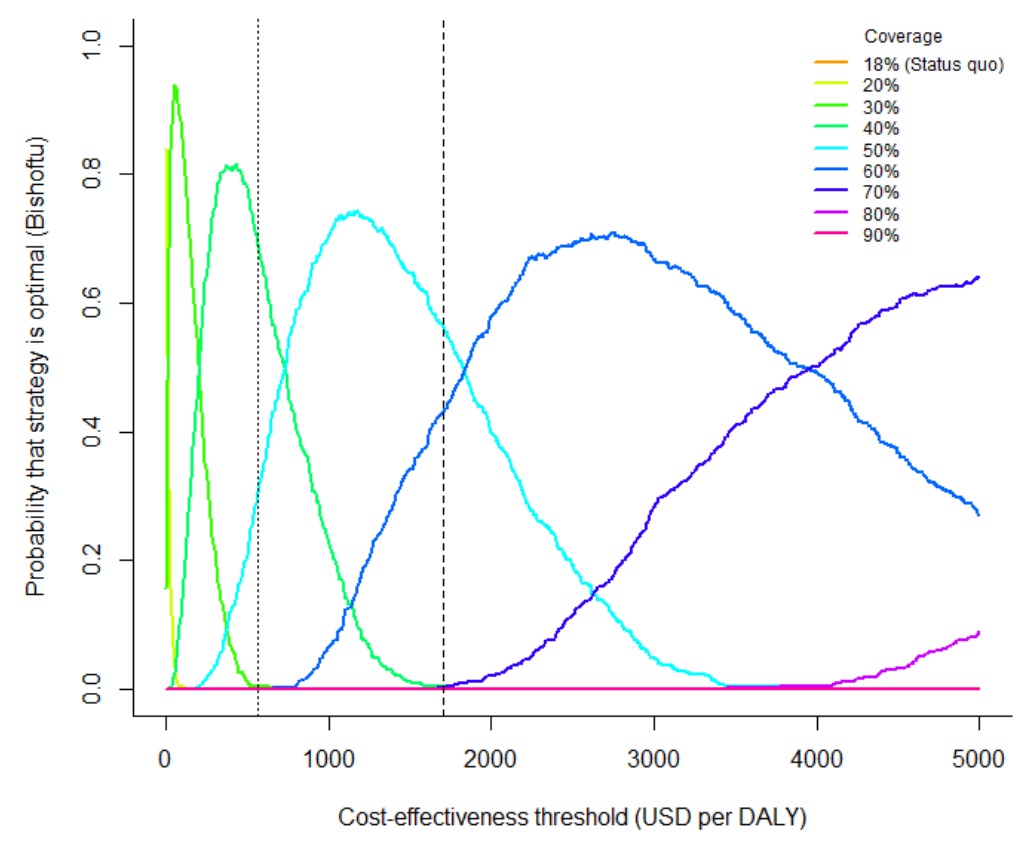

a) 


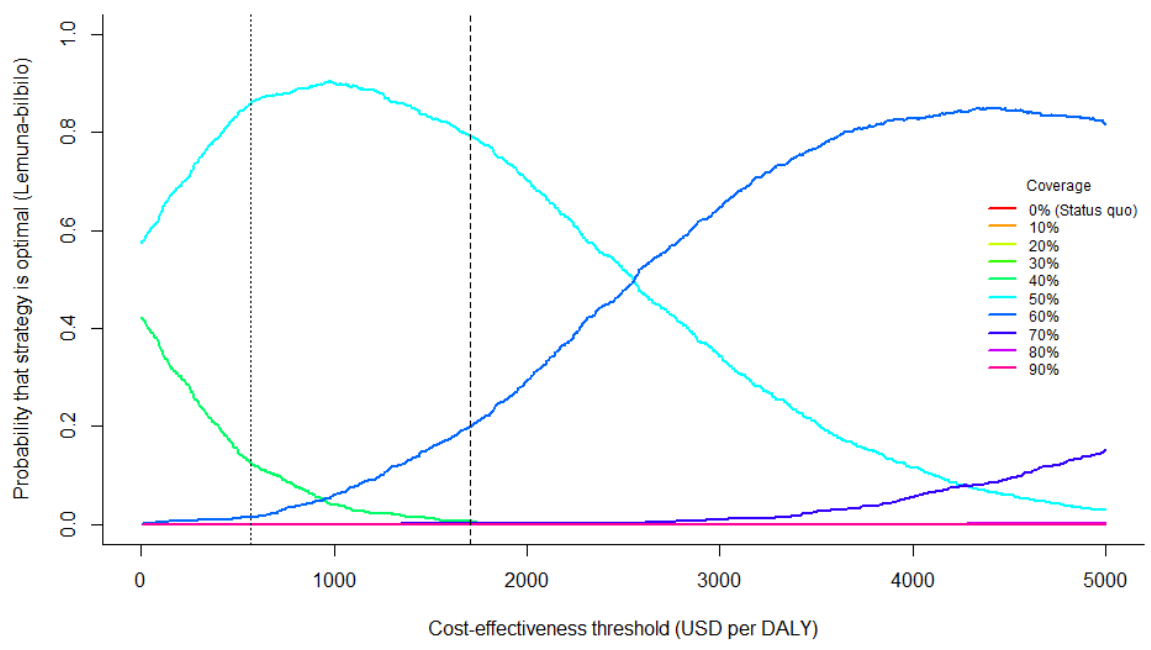

b)

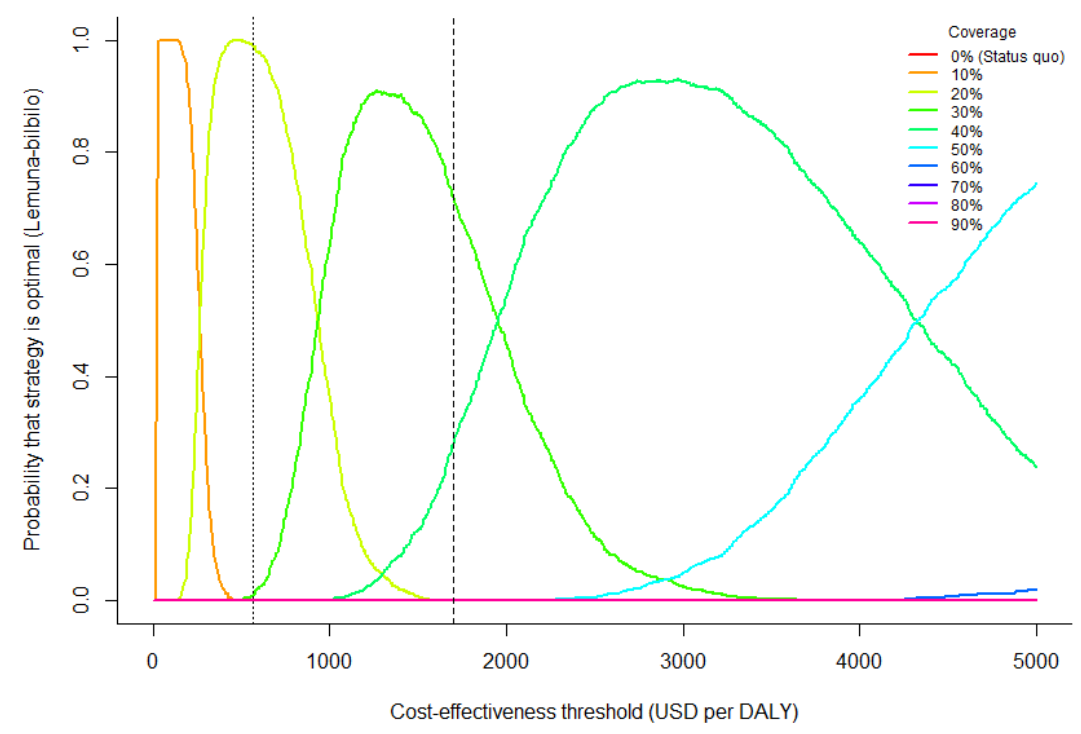

c)

Figure 7.4 Acceptability curves, showing the probability that each strategy would be optimal across a range of willingness-to-pay values for a) Bishoftu b) Lemuna-bilbilo district including cattle-related losses c) Lemuna-bilbilo district excluding cattle-related losses 


\section{Sensitivity and scenario analyses}

We investigated how the recommended strategy, i.e., the strategy most likely to be optimal at the cost-effectiveness threshold, would shift in response to changes in economic parameters. For Bishoftu, the recommended strategy was found to be insensitive to most economic parameters, including the number of dogs vaccinated per team, PET costs, and vaccination campaign training and advertising costs. However, if the price of a canine vaccine increases from a base case of $\$ 0.55$ to beyond $\$ 2$ per dog, the recommended coverage declines (see Supplementary information 7.1: Figure 2a). We found greater sensitivity to changes in economic parameters in Lemuna-bilbilo. Reducing the canine vaccine price to $\$ 0.1$ increases the recommended coverage to $60 \%$, and a price above $\$ 1.5$ reduces the recommended coverage to $40 \%$. The number of dogs vaccinated per day by a campaign team also influenced the recommended strategy. For instance, if less than 20 dogs are vaccinated per team per day, the recommended coverage declines to $40 \%$.

This recommended strategy in Lemuna-bilbilo was also sensitive to the herd-level cattle rabies incidence, and decreases to $40 \%$ coverage if the annual cattle herd level incidence drops from its current estimate of $20.9 \%$ to below $10 \%$. However, the recommended/optimal vaccination coverage in this district remains insensitive to changes in training and advertising costs, or in costs for post-exposure treatment (see Supplementary information 7.1: Figure 2b).

The two-way scenario analyses showed that a shorter time frame for analysis (5yrs) coupled with larger discounting rate (5\%) shifts the recommended strategy to $70 \%$ coverage in both districts (see Supplementary information 7.1: Figure 3a and b). A longer time frame (15yrs) and lower discount rate (1\%) did not change the recommended coverage of 50\% (Supplementary information 7.1: Figure 3c and 3d).

The scenario analysis around the recommended coverage for both lower (0.2) and higher (0.99) dog-to-human bite rates resulted in an unaltered recommended coverage of $50 \%$ for both districts (see Supplementary information 7.1: Figure 4a-d). The recommended coverage shifts to $70 \%$ and $90 \%$ in Bishoftu if rabid dogs are introduced at rates equivalent to $2 \%$ and $4 \%$ of current canine rabies, respectively, and to $90 \%$ for both scenarios in Lemuna-bilbilo (see Supplementary information 7.1: Figure 5a-d).

\subsection{Discussion}

We evaluated the cost-effectiveness of canine vaccination for reducing human rabies death, post-exposure treatment costs, and cattle rabies-related economic loss in Ethiopia. This costeffectiveness analysis is the first study to include cattle rabies-related losses, particularly important for livestock-dependent communities. Previous canine vaccination cost-effectiveness studies in Africa and elsewhere have focused primarily on the cost-effectiveness of preventing 
human rabies (Fitzpatrick et al., 2016; Shim et al., 2009; Fitzpatrick et al., 2014; Wera et al., 2016, Zinsstag et al., 2009; Dumas et al., 2015; Bilinski et al., 2016).

Cattle are one of the most important and numerous livestock species in sub-Saharan Africa including Ethiopia. They represent a major source of animal protein (dairy product and beef), provide draft power, thus support crop farming, are a source of cash, and fertilizer through manure, which is also used as fuel by some communities besides its socio-cultural roles (Mwai et al., 2015).

Given the difference among regions within Ethiopia with regard to canine demographics, and dependence on livestock for livelihoods, we considered control options tailored to specific types of human settlements, i.e. urban and rural districts. The inclusion of district specific inputs including dog demographics, human exposure cases, and campaign costs ensure that the recommended strategy will be impactful for each district.

In both districts, both canine and human rabies are expected to decline monotonically as vaccination coverage increases. Our analysis indicated that a canine vaccination coverage of $50 \%$ is the recommended strategy in both districts from a cost-effectiveness perspective. This coverage is lower than the WHO recommendation of 70\% coverage, which aims to eliminate rabies at without considering the costs of control (Coleman and Dye, 1996; WHO, 2013b). Our sensitivity analysis highlights, however, that the optimal coverage simulated over a shorter period of 5 years coincided with the coverage recommended by WHO. This result suggests that rabies control could be efficiently achieved by an intensive program over a shorter period of time, possibly followed by a less intensive (i.e. lower coverage) program sustained over a longer period of time, as has been suggested (Bogel and Meslin, 1990). A full analytical exploration and optimization of these combinations in Ethiopia or other resource-constrained contexts would be a valuable contribution to rabies control policy.

Surveys throughout Ethiopia have indicated that the current canine vaccination practices are far below this optimal coverage (Ali et al., 2010; Jemberu et al., 2013). In rural Lemuna-bilbilo, the current status quo of non-existent vaccination is costlier than any of the vaccination strategies evaluated, due to the high cattle rabies-related losses. This economic analysis provides a strong incentive for the immediate initiation of canine rabies vaccination, as the entire financial investment will be recouped. In urban Bishoftu, canine vaccination represents a financial outlay, but with a very efficient return on investment in terms of human health. In addition, as PEP is less accessible for most part of rural Ethiopia, canine vaccination is preferred to prevent human rabies. Canine vaccination is a one-time campaign (or at least scheduled periodically) based operation while PEP service requires continuous years round activity and cold chain for the vaccine as exposure can happen anytime. 
While we incorporated parameter uncertainty into our analysis whenever possible, it was necessary to make some parameter assumptions. Currently, there are no canine vaccination campaigns in Ethiopia from which we could extract epidemiological and economic data. This could make our cost estimations biased unpredictably. Further, survey-based human rabies case investigation can never be perfectly complete, as not all cases are reported to health centers and thus human exposures are likely be underestimated, and higher estimates of human exposure could justify greater vaccination coverage. However, coverage recommendations were robust as small deviations from the base case economic inputs did not change the cost-effective coverage. Of particular relevance, the Ethiopian government plans to replace the nervous tissue vaccine widely used as post-exposure prophylactic vaccine with safer cell culture vaccine, and there are speculations that the price could increase dramatically. Nevertheless, our analysis found that the recommended coverage remained insensitive to PET costs in both districts, which implying that the intended switch would not impact plans for canine vaccination coverage. Similarly, the recommended coverage was not sensitive to changes in the dog-to-human bite rate. This may be due to the fact that PET costs are small compared to the costs of vaccination programs.

The current practice of killing rabid dogs seen in Ethiopia supposed to reduce the public health burden (Deressa et al., 2010; Kabeta et al., 2015) but a lower (0.2) and higher (0.99) rabid dog human bite rates simulated in this study did not seem to change the recommended coverage from 50\%. This insensitivity could be due to the facts that PET costs, which are directly determined by the bite rate, represent an insignificant part of the total costs $(<3 \%)$. So, killing rabid dogs might reduce the number of rabid dogs as well as the number of human exposures but does not affect the optimal canine vaccination coverage.

We assumed a concerted vaccination campaign applied to all possible adjacent districts and no risk of reintroduction which otherwise require a vaccination campaign of larger coverage. In reality reintroduction of rabies from neighbouring areas is one of the potential challenges of rabies elimination (Fitzpatrick et al., 2012). Accordingly, sensitivity analysis around the optimal coverage revealed that a reintroduction at an equivalent of $2-4 \%$ of rabid cases increases the optimal vaccination coverage up to $90 \%$. This reintroduction could be from neighbouring districts and or wild animals. Though we didn't consider wild animals in the transmission dynamics of rabies as we had no data on the transmission between wildlife rabies, the assumption would not compromise the optimal coverage estimated in this study as it is unlikely for the wildlife contribution to change the dynamics significantly as less than $1 \%$ of human rabies exposures were from wild animals in Ethiopia (Beyene et al., 2017; Ramos et al., 2015).

Given that our analysis considered specific situations in Ethiopia, there are cost components that we conservatively did not consider. For example, the opportunity costs of dog owners to 
bring their dogs to vaccination center was not considered assuming that campaigns would be conducted door to door in rural districts and village level central-point based executed during weekends in urban districts where at least one person would be staying at home. If these costs were considered, the cost-effective coverage would be higher.

The recommended cost-effective annual coverage of 50\% in Bishoftu would require an average annual budget of $\$ 11,857$ over 10 years of which $99.8 \%$ goes to canine vaccination campaign costs and the rest to PET costs. Similarly, in the rural district/Lemuna-bilbilo, an optimal annual coverage of $50 \%$ would require on average $\$ 40,079$ annually over 10 years, of which $\$ 40,069$ for canine vaccination and the rest for PET. Knowing that Ethiopia has 529 districts, of which $423(80 \%)$ are rural districts and 106 (20\%) are urban districts, the estimate for a national annual canine vaccination budget would be $\$ 18.2$ million, which is far less than the national annual loss due to cattle rabies, which is estimated to be $\$ 209$ million (Jibat et al., 2016).

A concerted effort in implementing our recommended annual vaccination would significantly minimize, if not eliminate, rabid dogs, and consequently human exposure as well as livestockrelated loss within the first few years. This has also been demonstrated in many practical campaigns in Africa (Fitzpatrick et al., 2014; Zinsstag et al., 2009). However, in African region or East African sub-region, rabies elimination would require international and regional collaborations, without which elimination would not be possible to meet the 2030 target to eliminate dog mediated human rabies (WHO, 2015). Such studies on optimal cost-effective coverage are very useful in a resource-scarce setting as higher vaccination coverages don't necessarily give the best result. For instance in rural areas of Tanzania, vaccination coverages as high as $90 \%$ have been shown to have similar epidemiologic impacts as coverages at $70 \%$ (Bilinski et al., 2016), implying that vaccination with larger coverage may not pay back consistently.

\subsection{Conclusion}

Results from this study indicates that canine rabies vaccination in Ethiopia will be a costeffective measure against rabies by reducing the human health burden and by saving costs compared to the status quo of $18 \%$ canine vaccination coverage in urban districts and no vaccination in rural districts. With a primary focus on human health benefits (preventing human cases), the cost-effective canine vaccination coverages for the urban and rural district differed i.e. 50\% for the urban district and 30\% for rural the district. With the inclusion of the livestock rabies related losses in the rural district, the cost-effective coverage increased to $50 \%$. This highlights the relevance of applying a broad perspective of rabies burden with regard to the benefits of canine mass vaccination. The performed type of study is very useful in resourcescarce settings as higher vaccination coverages does not necessarily give the best result. For both districts, $50 \%$ coverage was identified as the most likely scenario to provide the greatest 
net health benefits at the WHO-recommended willingness-to-pay threshold over a time frame of 10 years.

\section{Acknowledgments}

The authors would like to thank Dr. Abraham Haile Kidane and Zoonosis research team at Ethiopian Public Health Institute (EPHI), the team at Ethiopian Ministry of Livestock and Fisheries Veterinary Services and Abishek Pandley at Yale School of Public Health for their contribution to this study. 


\subsection{References}

Ali, A., Mengistu, F., Hussen, K., Getahun, G., Deressa, A., Yimer, E., Tafese, K. 2010. Overview of Rabies in and around Addis Ababa, in Animals Examined in EHNRI Zoonoses Laboratory Between, 2003 and 2009. Ethiopian Veterinary Journal, 14(2), 91-101.

Bacar, N. 2011. Verhulst and the logistic equation (1838) in the book: A Short History of Mathematical Population Dynamics. 35-39.

Barton, G. R., Briggs, A. H., Fenwick, E. A. 2008. Optimal cost-effectiveness decisions: the role of the cost-effectiveness acceptability curve (CEAC), the cost-effectiveness acceptability frontier (CEAF), and the expected value of perfection information (EVPI). Value Health, 11(5), 886-897. doi:10.1111/j.1524-4733.2008.00358.x

Beyene, T. J., Kidane, A. H., Mourits, M. C., Hogeveen, H. 2017. Public health burden and exposure costs of rabies in Ethiopia. SVEPM conference, March 29-31, 2017. Inverness, Scotland, UK.

Bilinski, A. M., Fitzpatrick, M. C., Rupprecht, C. E., Paltiel, A. D., Galvani, A. P. 2016. Optimal frequency of rabies vaccination campaigns in Sub-Saharan Africa. Proceedings of the Royal Society B: Biological Sciences, 283(1842). doi:10.1098/rspb.2016.1211

Bogel, K., \& Meslin, F. X. 1990. Economics of human and canine rabies elimination: guidelines for programme orientation. Bulletin of the World Health Organization, 68(3), 281-291.

Briggs, A. H., Goeree, R., Blackhouse, G., O’Brien, B. J. 2002. Probabilistic analysis of costeffectiveness models: choosing between treatment strategies for gastroesophageal reflux disease. Medical decision making, 22(4), 290-308.

Brooks, S. P., \& Gelman, A. 1998. General methods for monitoring convergence of iterative simulations. Journal of computational and graphical statistics, 7(4), 434-455.

Carlo, C. M. 2004. Markov chain monte carlo and gibbs sampling. Notes,(April). 連結.

Coleman, P. G., Dye, C. 1996. Immunization coverage required to prevent outbreaks of dog rabies. Vaccine, 14(3), 185-186.

CSA. 2013. Population Projection of Ethiopia for All Regions At Wereda Level from 2014 2017. Federal Democratic Republic of Ethiopia Central Statistical Agency,, Addis Ababa, Ethiopia.

Deressa, A., Ali, A., Bayene, M., Selassie, B. N., Yimer, E., Hussen, K. 2010. The status of rabies in Ethiopia: A retrospective record review. Ethiopian Journal of Health Development, 24(2).

EHNRI. 2012. The Ethiopian Health and Nutrition Research Institute. Proceedings of The National Workshop on Rabies Prevention and Control in Ethiopia. October 18-19, 2012 Adama, Ethiopia.

Fitzpatrick, M. C., Hampson, K., Cleaveland, S., Meyers, L. A., Townsend, J. P., Galvani, A. P. 2012. Potential for Rabies Control through Dog Vaccination in Wildlife-Abundant Communities of Tanzania. Plos Neglected Tropical Diseases, 6(8), e1796. 
Fitzpatrick, M. C., Hampson, K., Cleaveland, S., Mzimbiri, I., Lankester, F., Lembo, T., . . . Galvani, A. P. 2014. Cost-effectiveness of canine vaccination to prevent human rabies in rural Tanzania. Annals of Internal Medicine, 160(2), 91-100.

Fitzpatrick, M. C., Shah, H. A., Pandey, A., Bilinski, A. M., Kakkar, M., Clark, A. D., . . . Galvani, A. P. 2016. One Health approach to cost-effective rabies control in India. Proceedings of the National Academy of Sciences, 113(51), 14574-14581. doi:10.1073/pnas.1604975113

Gelman, A., Rubin, D. B. 1992. Inference from iterative simulation using multiple sequences. Statistical science, 457-472.

Gsell, A. S., Knobel, D. L., Cleaveland, S., Kazwala, R. R., Vounatsou, P., Zinsstag, J. 2012. Domestic dog demographic structure and dynamics relevant to rabies control planning in urban areas in Africa: the case of Iringa, Tanzania. BMC Vet Res, 8(1), 236. doi:10.1186/1746-6148-8-236

Hampson, K., Coudeville, L., Lembo, T., Sambo, M., Kieffer, A., Attlan, M., . . . Global Alliance for Rabies Control Partners for Rabies, P. 2015. Correction: Estimating the global burden of endemic canine rabies. PLoS Negl Trop Dis, 9(5), e0003786. doi:10.1371/journal.pntd.0003786

Hampson, K., Dushoff, J., Cleaveland, S., Haydon, D. T., Kaare, M., Packer, C., Dobson, A. 2009. Transmission dynamics and prospects for the elimination of canine rabies. Plos Biology, 7(3), e53. doi:10.1371/journal.pbio.1000053

Hiby, L. R., Reece, J. F., Wright, R., Jaisinghani, R., Singh, B., Hiby, E. F. 2011. A markresight survey method to estimate the roaming dog population in three cities in Rajasthan, India. Bmc Veterinary Research, 7(1), 1-10. doi:10.1186/1746-6148-7-46

Hotez, P. J., Alvarado, M., Basáñez, M.-G., Bolliger, I., Bourne, R., Boussinesq, M., . . Budke, C. M. 2014. The global burden of disease study 2010: interpretation and implications for the neglected tropical diseases. PLoS Neg Tropl Diss, 8(7), e2865.

Jemberu, W. T., Molla, W., Almaw, G., Alemu, S. 2013. Incidence of Rabies in Humans and Domestic Animals and People's Awareness in North Gondar Zone, Ethiopia. PLoS Neg Trop Dis, 7(5), e2216.

Jha, P., Chaloupka, F. J., Moore, J., Gajalakshmi, V., Gupta, P. C., Peck, R., . . A Alleyne, G. 2006. Cost-Effectiveness Analysis for Priority Setting in Disease control priorities in developing countries. Disease control priorities in developing countries. 3rd Edition Working Paper \#2. Copenhagen Consensus 2012 Challenge Paper Diseases Chronic Disease Prevention and Control.

Jibat, T., Hogeveen, H., Mourits, M. C. M. 2015. Review on Dog Rabies Vaccination Coverage in Africa: A Question of Dog Accessibility or Cost Recovery? PLoS Negl Trop Dis, 9(2), e0003447. 
Jibat, T., Mourits, M. C., Hogeveen, H. 2016. Incidence and economic impact of rabies in the cattle population of Ethiopia. Prev Vet Med, 130, 67-76. doi:10.1016/j.prevetmed.2016.06.005.

Kabeta, T., Deresa, B., Tigre, W., Ward, M. P., Mor, S. M. 2015. Knowledge, Attitudes and Practices of Animal Bite Victims Attending an Anti-rabies Health Center in Jimma Town, Ethiopia. PLoS Negl Trop Dis, 9(6), e0003867.

Lavan, R. P., King, A. I. M., Sutton, D. J., Tunceli, K. 2017. Rationale and support for a One Health program for canine vaccination as the most cost-effective means of controlling zoonotic rabies in endemic settings. Vaccine, 35(13), 1668-1674. doi:http://dx.doi.org/10.1016/j.vaccine.2017.02.014.

Lembo, T., Hampson, K., Kaare, M. T., Ernest, E., Knobel, D., Kazwala, R. R., . . Cleaveland, S. 2010. The feasibility of canine rabies elimination in Africa: dispelling doubts with data. PLoS Negl Trop Dis, 4(2), e626.

Mathers, C., T, V., AD, L., J, S., ., E. M. e. 2001. National Burden of Disease Studies: A Practical Guide. Edition 2.0. Global Program on Evidence for Health Policy. Geneva: World Health Organization.

Mwai, O., Hanotte, O., Kwon, Y.-J., Cho, S. 2015. African Indigenous Cattle: Unique Genetic Resources in a Rapidly Changing World. Asian-Australasian Journal of Animal Sciences, 28(7), 911-921. doi:10.5713/ajas.15.0002R.

Okell, C. N., Pinchbeck, G. P., Stringer, A. P., Tefera, G., Christley, R. M. 2013. A communitybased participatory study investigating the epidemiology and effects of rabies to livestock owners in rural Ethiopia. Prev Vet Med, 108(1), 1-9. doi:10.1016/j.prevetmed.2012.07.003.

Plummer, M., Best, N., Cowles, K., Vines, K. 2008. coda: Output analysis and diagnostics for MCMC. $R$ package version 0.13-3, URL http://CRAN. R-project. org/package $=$ coda.

$\mathrm{R}$ core Team. 2014. The R project for statistical computing. Available at www. R-project. org/. Accessed October, 31, 2014.

Ramos, J. M., Melendez, N., Reyes, F., Gudiso, G., Biru, D., Fano, G., . . . Balcha, S. 2015. Epidemiology of animal bites and other potential rabies exposures and anti-rabies vaccine utilization in a rural area in Southern Ethiopia. Annals of Agricultural and Environmental Medicine, 22(1).

Shim, E., Hampson, K., Cleaveland, S., Galvani, A. P. 2009. Evaluating the cost-effectiveness of rabies post-exposure prophylaxis: a case study in Tanzania. Vaccine, 27(51), 71677172.

Soetaert, K., Petzoldt, T., Setzer, R. W. 2010. Package deSolve: solving initial value differential equations in R. J. Stat. Softw, 33, 1-25.

Tepsumethanon, V., Wilde, H., Meslin, F. X. 2005. Six criteria for rabies diagnosis in living dogs. J Med Assoc Thai, 88(3), 419-422. 
Wera, E., Mourits, M., Siko, M., Hogeveen, H. 2016. Cost-Effectiveness of Mass Dog Vaccination Campaigns against Rabies in Flores Island, Indonesia. Transboundary and Emerging Diseases. DOI: 10.1111/tbed.12590.

Wera, E., Velthuis, A. G. J., Geong, M., Hogeveen, H. 2013. Costs of Rabies Control: An Economic Calculation Method Applied to Flores Island. Plos One, 8(12), e83654.

WHO. 2013a. WHO Expert Consultation on Rabies. Second report. World Health Organization technical report series(982), 1.

WHO.2013b. WHO Expert Consultation on Rabies: second report: World Health Organization.WHO. (2014). WHO Guide for Rabies Pre and Post Exposure Prophylaxis in

Humans.

http://www.who.int/rabies/PEP_Prophylaxis_guideline_15_12_2014.pdf?ua=1.

Accessed date 6/1/2017

WHO.2010. Choosing Interventions That Are Cost-Effective (WHO-CHOICE). Geneva: World Health Organization;. Accessed at www.who.int/choice/en on 29/5/2017.

World Bank. 2015. GDP per capita for Ethiopia. http://data.worldbank.org/indicator/NY.GDP.PCAP.CD/countries/ET?display=graph access date- 3/9/2015.

WHO, 2005. WHO expert consultation on rabies: first report. WHO Technical Report Series 931. Geneva, Switzerland.

WHO. 2014. WHO Guide for Rabies Pre and Post Exposure Prophylaxis in Humans. http://www.who.int/rabies/PEP_Prophylaxis_guideline_15_12_2014.pdf?ua=1.

Accessed date 6/1/2017.

WHO 2015. Global elimination of dog-mediated human rabies: report of the rabies global conference, 10-11 December 2015, Geneva, Switzerland.

Yousaf, M. Z., Qasim, M., Zia, S., Rehman Khan, M. u., Ashfaq, U. A., Khan, S. 2012. Rabies molecular virology, diagnosis, prevention and treatment. Virology Journal, 9(1), 50. doi:10.1186/1743-422x-9-50.

Zinsstag, J., Durr, S., Penny, M. A., Mindekem, R., Roth, F., Gonzalez, S. M., . . Hattendorf, J. 2009. Transmission dynamics and economics of rabies control in dogs and humans in an African city. Proceedings of the National Academy of Sciences of the United States of America, 106(35), 14996-15001. doi:http://dx.doi.org/10.1073/pnas.0904740106. 


\section{Supplementary information 7.1}

\section{Transmission dynamics model equations}

$\frac{d_{s}}{d_{t}}=(1-v) * b *(S+E+V)-\beta * S * \frac{I}{K}-\gamma * S * N$

$\frac{d_{E}}{d_{t}}=\beta * S * I / K-\sigma * E-\gamma * E * N$

$\frac{d_{I}}{d_{t}}=\sigma * E-\alpha * I-\gamma * I * N$

$\frac{d_{V}}{d_{t}}=v * b *(S+E+V)-\gamma * V * N$

$\frac{d_{N t}}{d_{t}}=\frac{d_{s}}{d_{t}}+\frac{d_{e}}{d_{t}}+\frac{d_{I}}{d_{t}}+\frac{d_{V}}{d_{t}}$

\section{Dog demographics}

In April and May 2015, we conducted a dog population survey in two districts representing urban and rural highland districts namely Bishoftu and Lemuna-bilbilo districts, respectively (detailed results of the survey will be published separately). Following recommended guidelines for surveying roaming dog population by World Society for Animal Protection (WSAP, 2009) we systematically selected 13 non-contagious wards bounded by roads in Bishoftu town district and 12 non-contagious wards in Lemuna-bilbilo district. We then adopted a sight-mark-resight approach for studying population abundance and modified it to markresight as previously applied by Hiby and colleagues (Hiby et al., 2011). The dog counting (resight) was done for both restricted and freely roaming dogs twice after marking. For estimation of dog density (i.e. carrying capacity, $\mathrm{K} / \mathrm{km}^{2}$ ), the logit-normal mixed effects models were carried out in the freely available Program MARK, version 6.2, developed by Gary White, Colorado State University (http://www.phidot.org/software/mark/docs/book/) (White, 2017). District specific sex ratios (h) were derived from a dog demography survey. We were, however, not able to collect accurate information on average litter size (l), and litter frequency (/) and pup survival (s) estimates. Due to the potential similarities of agro-ecologies of Tanzania and Ethiopia, we adopted these missing parameter published from Tanzania study (Hampson et al., 2009). The per capita birth rate (b) was assumed to be the product of the sex ratio (h), the average litter size (l), and frequency (f) and pup survival (s) (b =hlfs) (Hampson et al., 2009).

\section{Human rabies exposure data collection and contact tracing}

A retrospective case study was conducted to compile data on the incidence of human rabies exposure over the period of one year (September 2013 to August 2014) through an extensive animal bite case search in the two districts of Ethiopia. The bite victims were traced using data collected from the recorded cases at health centers as well as by information obtained from questioning the local community to trace unregistered bite cases. After the tracing both 
registered and unregistered cases, victims (or their family) were contacted and questioned about their use of PET, the incurred costs, the behavioral manifestation of the biting animal and if the victim died after the bite. Based on these data the health burden of people who died after being bitten by potentially rabid dogs was assessed as well as the costs of the applied post-exposure treatment were estimated (detailed description of the human health burden and costs of treatment following rabies exposure is published elsewhere (Beyene et al., 2017).

Table 1 Rabies transmission dynamics model parameters, distribution, prior/initial input estimates

\begin{tabular}{|c|c|c|c|c|c|}
\hline $\begin{array}{l}\text { Parameter } \\
\text { s }\end{array}$ & Description & Distribution & $\begin{array}{l}\text { Estimate } \\
\text { Bishoft } \\
\text { u/ } \\
\text { Urban }\end{array}$ & $\begin{array}{l}\text { Lemuna- } \\
\text { bilbilo/Rur } \\
\text { al }\end{array}$ & Source \\
\hline$\beta$ & $\begin{array}{l}\text { Dog to dog bite } \\
\text { rate/day }\end{array}$ & normal & 0.42 & 0.4 & $\begin{array}{l}\text { (Hampson et al., } \\
\text { 2009) }\end{array}$ \\
\hline $\mathrm{K}$ & $\begin{array}{l}\text { Carrying capacity } \\
\left(\text { dogs } / \mathrm{km}^{2}\right)\end{array}$ & logistic & 340 & 35.4 & Field survey \\
\hline $\mathrm{b}$ & Birth rate/day & normal & 0.0015 & 0.0011 & Field survey \\
\hline $1 / \sigma$ & $\begin{array}{l}\text { Incubation period } \\
\text { (in days) }\end{array}$ & normal & 22.3 & 22.3 & $\begin{array}{l}\text { (Hampson et al., } \\
\text { 2009) }\end{array}$ \\
\hline $1 / \alpha$ & $\begin{array}{l}\text { Infectious period } \\
\text { (in days) }\end{array}$ & gamma & 3.1 & 3.1 & $\begin{array}{l}\text { (Hampson et al., } \\
\text { 2009) }\end{array}$ \\
\hline \multirow[t]{2}{*}{$\mathrm{v}$} & $\begin{array}{l}\text { Vaccination } \\
\text { coverage status quo }\end{array}$ & & 0 & $18 \%$ & Field survey \\
\hline & Dog density/km2 & normal & 340 & 35.5 & Field survey \\
\hline \multirow[t]{4}{*}{$\delta$} & $\begin{array}{l}\text { Human bite } \\
\text { rate/rabid dog }\end{array}$ & normal & 0.51 & 0.51 & $\begin{array}{l}\text { (Hampson et al., } \\
\text { 2009) }\end{array}$ \\
\hline & Human density $/ \mathrm{km}^{2}$ & & 3500 & 158 & (CSA, 2013) \\
\hline & $\begin{array}{l}\text { Human } \\
\text { exposure/year }\end{array}$ & & 189 & 189 & $\begin{array}{l}\text { (Beyene et al., } \\
\text { 2017) }\end{array}$ \\
\hline & $\begin{array}{l}\text { Number of human } \\
\text { deaths/year }\end{array}$ & & 1 & 8 & $\begin{array}{l}\text { (Beyene et al., } \\
\text { 2017) }\end{array}$ \\
\hline$p$ & $\begin{array}{l}\text { Probability to } \\
\text { receive sufficient } \\
\text { doses of PEP* }\end{array}$ & Beta & 0.77 & 0.5 & $\begin{array}{l}\text { (Beyene et al., } \\
\text { 2017)) }\end{array}$ \\
\hline \multirow[t]{2}{*}{$m$} & $\begin{array}{l}\text { Probability of death } \\
\text { due to rabies }\end{array}$ & Multinomial & 0.16 & 0.15 & $\begin{array}{l}\text { (Beyene et al., } \\
\text { 2017) } \\
\text { (Shim et al., } \\
\text { 2009) }\end{array}$ \\
\hline & $\begin{array}{l}\text { District size (in } \\
\mathrm{km}^{2} \text { ) }\end{array}$ & & 40 & 1184 & $\begin{array}{l}\text { (Beyene et al., } \\
\text { 2017) }\end{array}$ \\
\hline
\end{tabular}

PEP*=Post-Exposure Prophylaxis 


\section{Cost estimation for dog vaccination campaigns}

The costs of rabies control by dog mass vaccination campaign were estimated by modifying an economic model developed by Wera et al., (2013). Accordingly, the costs of mass canine vaccination (CMCV) campaign include costs of the vaccine $\left(\mathrm{C}_{v a}\right)$, costs of consumables (needle and syringe, ice bar, disinfectant and swab, certificate and collar) $\left(C_{c o}\right)$, costs of temporary vaccinators and supervisors (perdium and transportation) $\left(C_{v s}\right)$, costs of training vaccinators and costs of information/advertising the campaign $\left(\mathrm{C}_{\mathrm{ti}}\right)$, capital costs (refrigerator, muzzle and cool bags) $\left(C_{c c}\right)$. The total costs of mass dog vaccination given by Equation 1 .

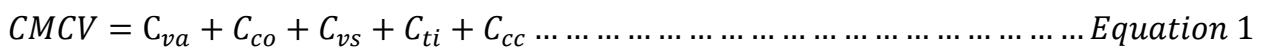

To estimate the total campaign costs under each vaccination coverage strategy, the costs of vaccine and consumables which were estimated per vaccinated dog and multiplied by the number of dogs to be vaccinated under each vaccination coverage/strategy as predicted by the transmission model simulation. Costs of vaccinators and supervisors were estimated for the number of days required to vaccinate the required number of dogs under each strategy. While costs of training vaccinators and information/advertising the campaign and capital costs were assumed to be same irrespective of the vaccination coverage strategy. The vaccination campaign is assumed to be at village level central point in urban (Bishoftu district) and door-to-door in rural (Lemuna-bilbilo district).

\section{Costs of the vaccine}

Costs of the vaccine, $C_{v a}$ depend on the price of the vaccine per dose $\left(p_{v a}\right)$, costs of transportation of the vaccine from manufacturer to each village $\left(t_{v a}\right)$, and the number of dogs to be vaccinated $\left(n_{d v}\right)$ under each strategy (Equation 2$)$. The rabies vaccine was supposed to be sourced from the National Veterinary Institute in Ethiopia and assured to confer protection for at least one year (personal communication with Dr Berecha Bayissa, Rabies vaccine production department head at National Veterinary Institute, Debre zeit, Ethiopia).

$C_{v a}=\left(p_{v a}+t_{v a}\right) \times n_{d v}$ Equation 2

\section{Costs of consumables}

Costs of consumables, $C_{c o}$ depends on the price of syringes and needles $\left(P_{s n}\right)$, ice bars $\left(P_{i b}\right)$ required per vaccinated dog per day, disinfectant and swabs $\left(P_{d s}\right)$, price of collar $\left(P_{c l}\right)$ and price of certificate $\left(P_{c r}\right)$ (Equation 3). A collar to distinguish between a vaccinated and an unvaccinated dog was assumed to be applied at the vaccination spot. 
$C_{c o}=\left(P_{s n}+\left(\frac{P_{i b}}{n_{c a p v}}\right)+P_{d s}+P_{c l}+P_{c r}\right) \times n_{d v}$ Equation 3

where $n_{\text {capv }}$ is the number of dogs to be vaccinated by one vaccinator team per day. The vaccination of dogs was assumed to be administered by a team of temporary vaccinators under close supervision of a veterinarian. A recent central point vaccination campaign in Malawi recorded that a team of vaccinators was able to vaccinate150 owned dogs daily at most (Gibson et al., 2016), supporting the assumption of a four-person vaccination team being able to vaccinate as many as 75 dogs daily in a rural area and 150 in an urban district.

\section{Costs of vaccinators and supervisors}

Costs of vaccinators, $C_{v s}$ consist of the costs for temporary vaccinators $\left(C_{t v}\right)$ and costs for supervisor of the vaccinators, $C_{s v}$ (Equation 4).

$C_{v s}=C_{t v}+C_{s}$

Equation 4

Where, $C_{t v}$ were calculated per day by the number of vaccination days required to vaccinate the dogs estimated under each vaccination strategy $\left(n_{v \text { days }}\right)$ multiplied by the number of vaccination villages or teams $\left(n_{v v}\right)$, the daily rate (perdium) for temporary vaccinators in the team $\left(P_{t v}\right)$, the number of vaccinator persons per vaccination team $\left(v_{t}\right)$ and the costs of fuel per vaccination team per day $\left(f_{t v}\right)$ (Equation 5$)$.

$C_{t v}=n_{v d a y s} \times\left(\left(P_{t v} \times v_{t}\right)+f_{t v}\right) \times n_{v v}$ Equation 5

Costs for supervisors, $\mathrm{C}_{\mathrm{sv}}$ were calculated based on the number of vaccination days $\left(n_{v \text { days }}\right)$, the perdium for a supervisor $\left(P_{s p}\right)$ and the fuel costs for a supervisor per day $\left(f_{s p}\right)$ (Equation 6).

$C_{s v}=n_{\text {vays }} \times\left(P_{s p}+f_{s p}\right)$ Equation 6

The number of vaccination days $\left(n_{v \text { days }}\right)$ was estimated based on the assumption that each village is visited by one vaccination team. Thus, the number of vaccination days equals the total number of dogs to be vaccinated $\left(n_{v d}\right)$ under each vaccination strategy divided by the number of dogs vaccinated each day across the number of villages $\left(n_{v}\right)$ (Equation 7$)$.

Operationally, for both districts, we assumed that vaccination campaigns with a coverage higher than 50\% require extra "dog search days", incurring extra costs for vaccinators and supervisors. We considered 1, 2, 3, and 4 extra days for a coverage of $60 \%, 70 \%, 80 \%$ and $90 \%$, respectively. In addition, for Lemuna-bilbilo, an increase in vaccination time (e.g. travel time) 
per dog was considered for coverages lower than $50 \%$, as fewer dogs per square kilometre can be covered to ensure an even geographic distribution of vaccinated dogs. Based on this assumption 4, 3, 2 and 1 additional days were considered to realise a coverage of $10 \%$, 20\%, $30 \%$ and $40 \%$, respectively. Along with an increase in travel time, the transportation costs of the vaccination team $\left(f_{t v}\right)$ were considered to be increased with a factor 4 per coverage between $10 \%$ and $40 \%$.

The number of vaccination days $\left(\mathrm{n}_{\mathrm{v} d a y s}\right)$ for supervisors was extended by $50 \%$ for each coverage as they complete documentations and reports following the campaign and same additional transportation cost was also applied in both districts.

$n_{\text {vays }}=n_{v d} /\left(n_{\text {capv }} \times n_{v}\right)$

Equation 7

\section{Costs of training vaccinators and costs of information/advertising the campaign $\left(C_{t i}\right)$}

Costs of training vaccinators and costs of information/advertising the campaign $\left(\mathrm{C}_{t i}\right)$ consist of the costs of meeting and training temporary vaccinators and costs of information campaign through printing and distribution of the leaflets and posters, and the development and broadcast of radio and television advertisements. We assumed a lump sum of 2000 Ethiopian birr (ETB) assuming 1000 ETB (72 USD) for training vaccinators and 1000 ETB (72 USD) for information/advertising the campaign per village.

\section{Capital costs}

Capital costs, $C_{c c}$ includes the yearly depreciation costs for cool bags, refrigerators, and muzzles. We assumed the same capital cost for all mass dog coverage strategies irrespective of the number of vaccination days in use (Equation 8).

$C_{c c}=\left(\left(n_{c b} \times p_{c b}+n_{r f} \times P_{r f}+n_{m z} \times p_{m z}\right) / l_{c m r}\right)$ Equation 8

Where $n_{c b}$ is the number of cool bags needed each year per district accounting for the number of teams, $p_{c b}$ the price of a cool bag, $n_{r f}$ the number of refrigerators per district, $P_{r f}$ the price of a refrigerator, $n_{m z}$ the number of muzzles, $p_{m z}$ the price of a muzzle, and $l_{c m r}$ the number of life years of capital goods (cool bags, refrigerators, and muzzle). For simplification, we assumed the number of life years of capital goods to be 5 years and the salvage value afterwards equal to zero. 
Table 2 Economic input parameters used in estimating the costs of dog vaccination campaigns

\begin{tabular}{|c|c|c|c|c|c|}
\hline \multirow[t]{2}{*}{$\begin{array}{l}\text { Param } \\
\text { eters }\end{array}$} & \multirow[t]{2}{*}{ Description } & \multirow[t]{2}{*}{$\begin{array}{l}\text { Type of } \\
\text { distribution }\end{array}$} & \multicolumn{2}{|c|}{$\begin{array}{l}\text { Value } \\
(95 \% \mathrm{CI})\end{array}$} & \multirow[t]{2}{*}{ Source } \\
\hline & & & Urban & Rural & \\
\hline $\mathrm{C}_{\mathrm{va}}$ & Cost of rabies vaccine (in USD) & Point & 0.56 & 0.56 & NVI \\
\hline $\mathrm{T}_{\mathrm{va}}$ & $\begin{array}{l}\text { Cost of transporting a dose of vaccine } \\
\text { to a district (in USD) }\end{array}$ & $\begin{array}{l}\text { truncated } \\
\text { normal }\end{array}$ & 0.35 & 0.09 & Market survey \\
\hline $\mathrm{P}_{\mathrm{sn}}$ & $\begin{array}{l}\text { Cost of syringe, needle, certificate, } \\
\text { and collar (in USD) }\end{array}$ & $\begin{array}{l}\text { truncated } \\
\text { normal }\end{array}$ & 0.55 & 0.52 & Market survey \\
\hline $\mathrm{P}_{\mathrm{ib}}$ & $\begin{array}{l}\text { Cost of ice bar for keeping vaccine } \\
\text { cold in icebox (in USD) }\end{array}$ & $\begin{array}{l}\text { truncated } \\
\text { normal }\end{array}$ & 0.93 & 0.93 & Market survey \\
\hline $\mathrm{n}_{\text {capv }}$ & Number of dogs vaccinated/day/team & triangular* & 150 & 75 & Expert opinion \\
\hline $\mathrm{n}_{\mathrm{vv}}$ & Number of vaccination villages & Point & 9 & 27 & assumption \\
\hline $\mathrm{P}_{\mathrm{tv}}$ & $\begin{array}{l}\text { Daily perdium rate of temporary } \\
\text { vaccinators (in USD) }\end{array}$ & $\begin{array}{l}\text { truncated } \\
\text { normal }\end{array}$ & 6.9 & 6.9 & Survey \\
\hline $\mathrm{P}_{\mathrm{sp}}$ & $\begin{array}{l}\text { Daily perdium rate of supervisors (in } \\
\text { USD) }\end{array}$ & $\begin{array}{l}\text { truncated } \\
\text { normal }\end{array}$ & 7.8 & 7.8 & Survey \\
\hline $\mathrm{v}_{\mathrm{t}}$ & Number of vaccinators per team & Point & 4 & 4 & assumption \\
\hline $\mathrm{f}_{\mathrm{tv}}$ & $\begin{array}{l}\text { Cost of fuel for temporary } \\
\text { vaccinators per day (in USD) }\end{array}$ & $\begin{array}{l}\text { truncated } \\
\text { normal }\end{array}$ & 9.3 & 18.5 & Survey \\
\hline $\mathrm{f}_{\mathrm{sp}}$ & $\begin{array}{l}\text { Cost of fuel for supervisors } \\
\text { vaccinators per day (in USD) }\end{array}$ & $\begin{array}{l}\text { truncated } \\
\text { normal }\end{array}$ & 20.1 & 37.1 & Survey \\
\hline $\mathrm{C}_{\mathrm{ti}}$ & $\begin{array}{l}\text { Cost of training and campaign, lump } \\
\text { sum (in USD) }\end{array}$ & Point & 833 & 2500 & Assumption \\
\hline$n_{c b}$ & Number of cool bags required & Point & 9 & 27 & Assumption \\
\hline$p_{r f}$ & Number of refrigerators required & Point & 9 & 27 & Assumption \\
\hline$p_{m z}$ & Number of muzzles required & Point & 9 & 27 & Assumption \\
\hline Lcmr & Lifetime of capital goods & Point & 5 & 5 & Assumption \\
\hline$p_{c b}$ & Price per cool bag (in USD) & $\begin{array}{l}\text { truncated } \\
\text { normal }\end{array}$ & 23.15 & 23.15 & Market survey \\
\hline$P_{r f}$ & Price per refrigerator (in USD) & $\begin{array}{l}\text { truncated } \\
\text { normal }\end{array}$ & 694.4 & 694.44 & Market survey \\
\hline$p_{m z}$ & Price per muzzle (USD) & $\begin{array}{l}\text { truncated } \\
\text { normal }\end{array}$ & 23.1 & 23.1 & Market survey \\
\hline
\end{tabular}


Table 3 Health burden parameters, distributions and input estimates

\begin{tabular}{|c|c|c|c|c|c|}
\hline \multirow[t]{2}{*}{ Parameters } & \multirow[t]{2}{*}{ Description } & \multirow{2}{*}{$\begin{array}{l}\text { Type of } \\
\text { distribution }\end{array}$} & \multicolumn{2}{|c|}{ Values } & \multirow[t]{2}{*}{ Source } \\
\hline & & & Urban & Rural & \\
\hline PET cost & $\begin{array}{l}\text { Cost of PET per sufficient } \\
\text { PEP dose (in USD) }\end{array}$ & Beta & 23.2 & 30.4 & $\begin{array}{l}\text { (Beyene et al., } \\
\text { 2017) }\end{array}$ \\
\hline${ }^{1} \mathrm{P} 1$ & $\begin{array}{l}\text { Likelihood of a suspected } \\
\text { rabid dog biting a person }\end{array}$ & multinomial & 0.5 & 0.5 & $\begin{array}{l}\text { Computation } \\
\text { after Hampson } \\
\text { et al., } 2009\end{array}$ \\
\hline P2 & $\begin{array}{l}\text { Likelihood of a suspected } \\
\text { rabid dog bite victim visit } \\
\text { health centre and receive } \\
\text { sufficient doses of PEP }\end{array}$ & multinomial & 0.85 & 0.55 & $\begin{array}{l}\text { Computation } \\
\text { after } \\
\text { (Beyene et al., } \\
\text { 2017) }\end{array}$ \\
\hline${ }^{1} \mathrm{P} 3$ & $\begin{array}{l}\text { Likelihood of victim not } \\
\text { receiving PEP and dying } \\
\text { due to rabies }\end{array}$ & multinomial & 0.16 & 0.16 & $\begin{array}{l}\text { Computation } \\
\text { after (Beyene } \\
\text { et al., 2017) }\end{array}$ \\
\hline $\begin{array}{l}\text { DALY per } \\
\text { human rabies } \\
\text { case }\end{array}$ & $\begin{array}{l}\text { Average DALY per } \\
\text { human rabies case }\end{array}$ & multinomial & 46.7 & 47.7 & $\begin{array}{l}\text { Computation } \\
\text { after (Beyene } \\
\text { et al., 2017) }\end{array}$ \\
\hline $\begin{array}{l}\text { DALY per } \\
\text { rabid dog }\end{array}$ & $\begin{array}{l}\mathrm{P} 1 *(1- \\
\mathrm{P} 2) * \mathrm{P} 3 * \mathrm{DALY}(\text { human })\end{array}$ & & 0.64 & 1.67 & Computation \\
\hline $\begin{array}{l}\text { Costs of PET } \\
\text { per rabid dog }\end{array}$ & $\begin{array}{l}\mathrm{P} 1 * \mathrm{P} 2 * \text { PET costs (in } \\
\text { USD) }\end{array}$ & & 9.86 & 10.79 & $\begin{array}{l}\text { Computation } \\
\text { after (Beyene } \\
\text { et al., 2017) }\end{array}$ \\
\hline
\end{tabular}

Table 4 Parameters and inputs used to estimate economic loss related to livestock rabies (for rural district only)

\begin{tabular}{llll}
\hline Parameters & Description & Value & Source \\
\hline$H_{\text {inc }}$ & Herd level rabies incidence (\%) & 19.4 & (Jibat et al., 2016) \\
$N_{\text {herd }}$ & Cattle per herd & 14.5 & (Jibat et al., 2016) \\
$L_{\text {pah }}$ & Loss per affected herd & 147 & (Jibat et al., 2016) \\
\hline
\end{tabular}




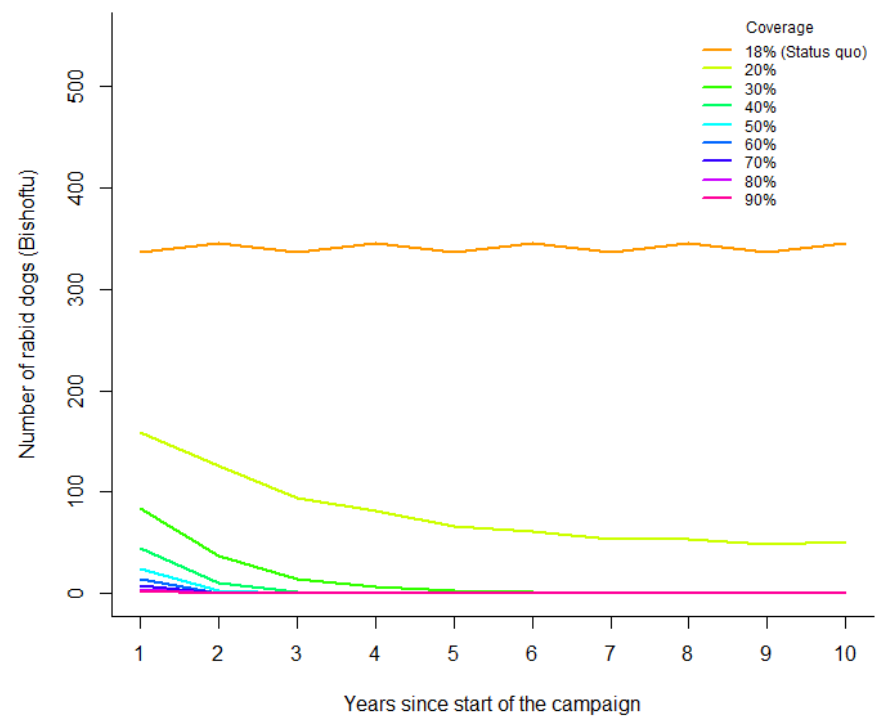

a)

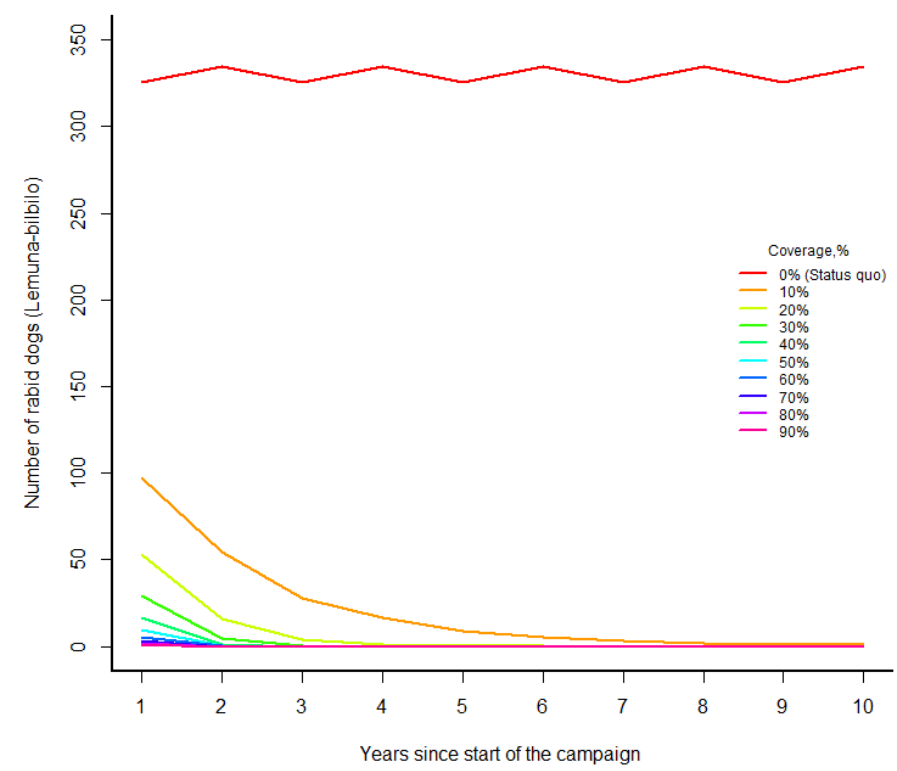

b) 


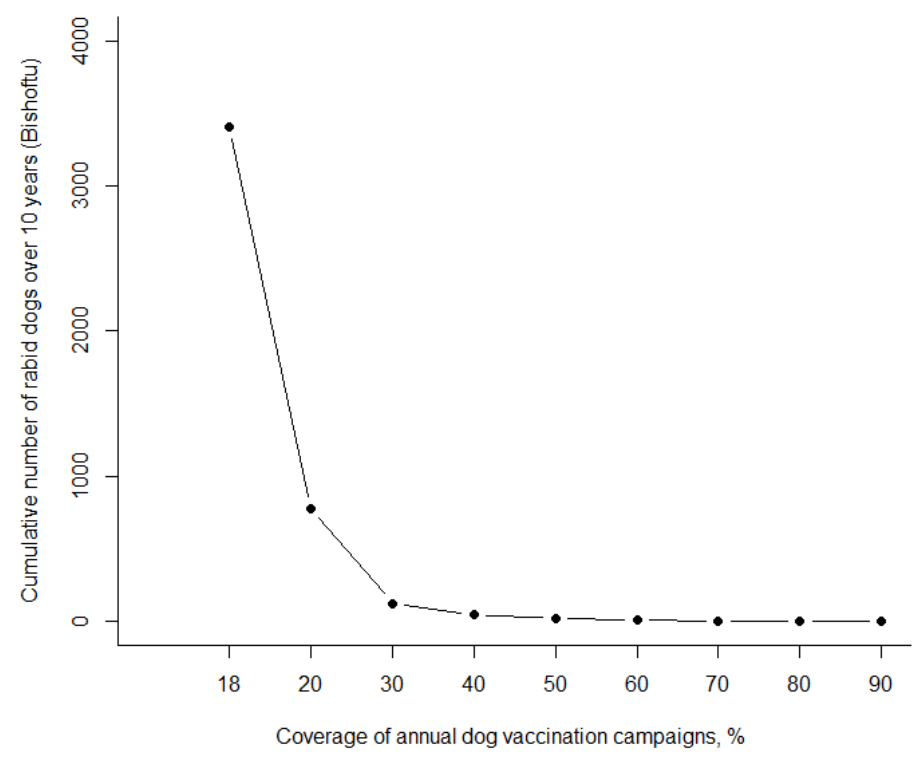

c)

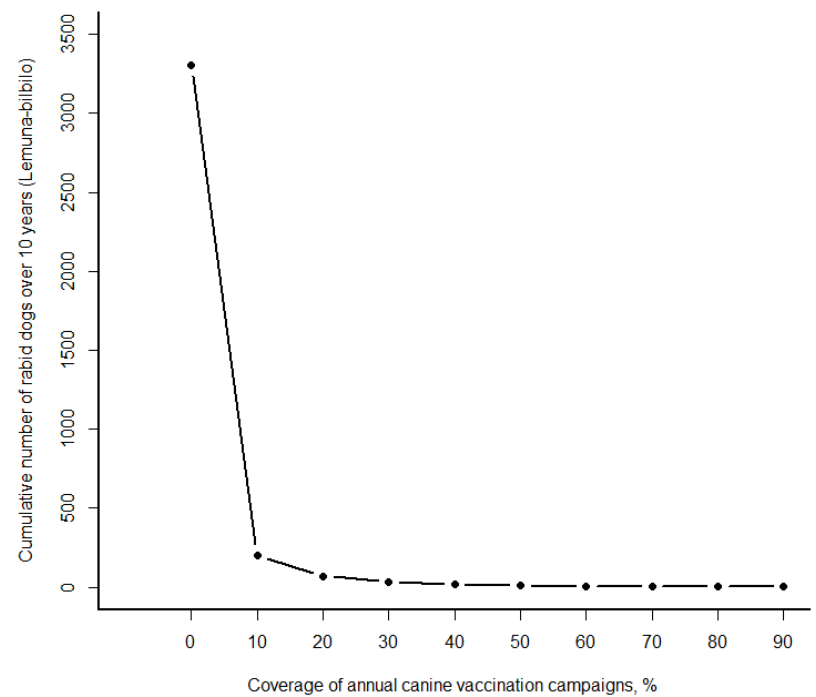

d) 


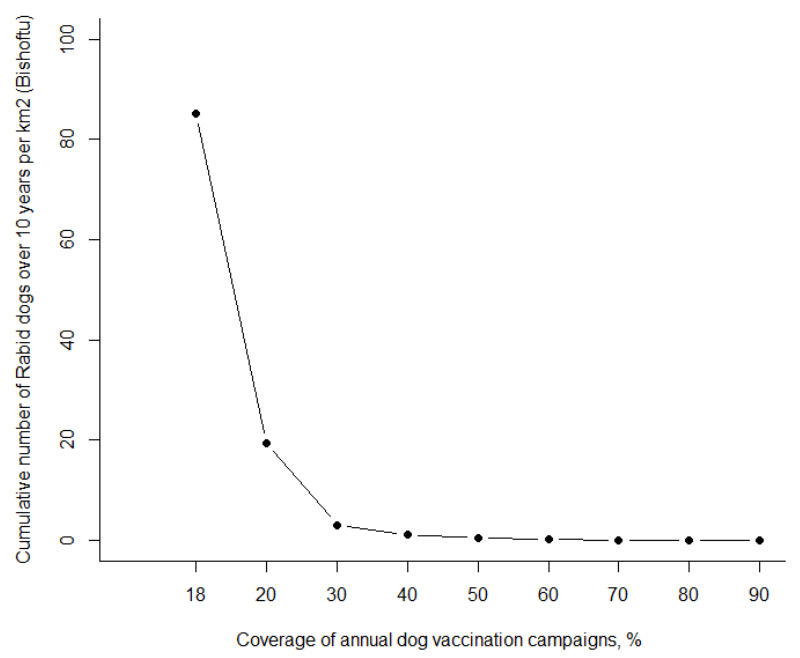

e)

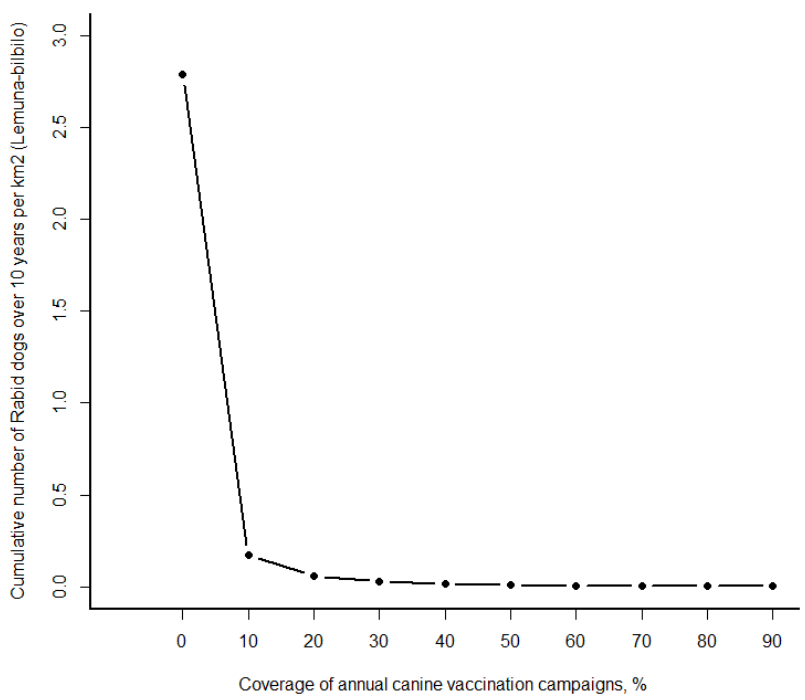

f)

Figure 1: Total number of rabid dogs over 10 years for each vaccination coverage a) Bishoftu b) Lemuna-bilbilo, cumulative number of rabies dogs over a 10-year in c) Bishoftu d) Lemunabilbilo, and cumulative number of rabies dogs per $\mathrm{km}^{2}$ in e) Bishoftu and f) Lemuna Bilbilo under different canine vaccination coverage strategies 


\begin{tabular}{|l|c|c|c|c|c|c|c|}
\hline Vaccine price (\$) & 0.1 & $\mathbf{0 . 5 5 *}$ & 1 & 1.5 & 2 & 2.5 & 3 \\
\hline & $50 \%$ & $50 \%$ & $50 \%$ & $50 \%$ & $40 \%$ & $40 \%$ & $40 \%$ \\
\hline Dogs vaccinated/day/team & 25 & 50 & 100 & $\mathbf{1 5 0}$ & 200 & 250 & 300 \\
\hline & $50 \%$ & $50 \%$ & $50 \%$ & $50 \%$ & $50 \%$ & $50 \%$ & $50 \%$ \\
\hline PET cost (\$) & 10 & $\mathbf{2 3}$ & 50 & 75 & 100 & 150 & 200 \\
\hline & $50 \%$ & $50 \%$ & $50 \%$ & $50 \%$ & $50 \%$ & $50 \%$ & $50 \%$ \\
\hline $\begin{array}{l}\text { Canine vaccination campaign } \\
\text { training and advertisement cost (\$) }\end{array}$ & 100 & $\mathbf{1 0 0 *}$ & 2000 & 4000 & 8000 & 16000 & 32000 \\
\hline
\end{tabular}

a) Urban /Bishoftu district

\begin{tabular}{|l|l|l|l|l|l|l|l|}
\hline & 0.1 & $\mathbf{0 . 5}$ & 1 & 1.5 & 2 & 2.5 & 3 \\
\hline & $60 \%$ & $50 \%$ & $50 \%$ & $40 \%$ & $40 \%$ & $40 \%$ & $40 \%$ \\
\hline Vaccine price (\$) & 10 & 20 & 35 & $75 *$ & 105 & 140 & 175 \\
\hline & $40 \%$ & $40 \%$ & $50 \%$ & $50 \%$ & $50 \%$ & $50 \%$ & $50 \%$ \\
\hline PET cost (\$) & 5 & 10 & 20 & $\mathbf{3 0}$ & 50 & 60 & 80 \\
\hline Canine vaccination campaign & $50 \%$ & $50 \%$ & $50 \%$ & $50 \%$ & $50 \%$ & $50 \%$ & $50 \%$ \\
\hline training and advertisement cost (\$) & 100 & $\mathbf{2 5 0 *}$ & 5000 & 10000 & 20000 & 40000 & 80000 \\
\hline & $50 \%$ & $50 \%$ & $50 \%$ & $50 \%$ & $50 \%$ & $50 \%$ & $50 \%$ \\
\hline Herd level incidence (\%) & 0 & 5 & 10 & 15 & $\mathbf{2 0} *$ & 30 & 40 \\
\hline
\end{tabular}

b) Rural/ Lemuna-bilbilo

Figure 2: One-way sensitivity analysis on the impact of economic parameters on recommended rabies vaccination coverage for a) Bishoftu b) Lemuna-bilbilo district. Values bolded with asterisk are base case values. Vaccination coverage with colour gradient goes from $60 \%$ coverage (dark red) to light $40 \%$ coverage (light pink) 


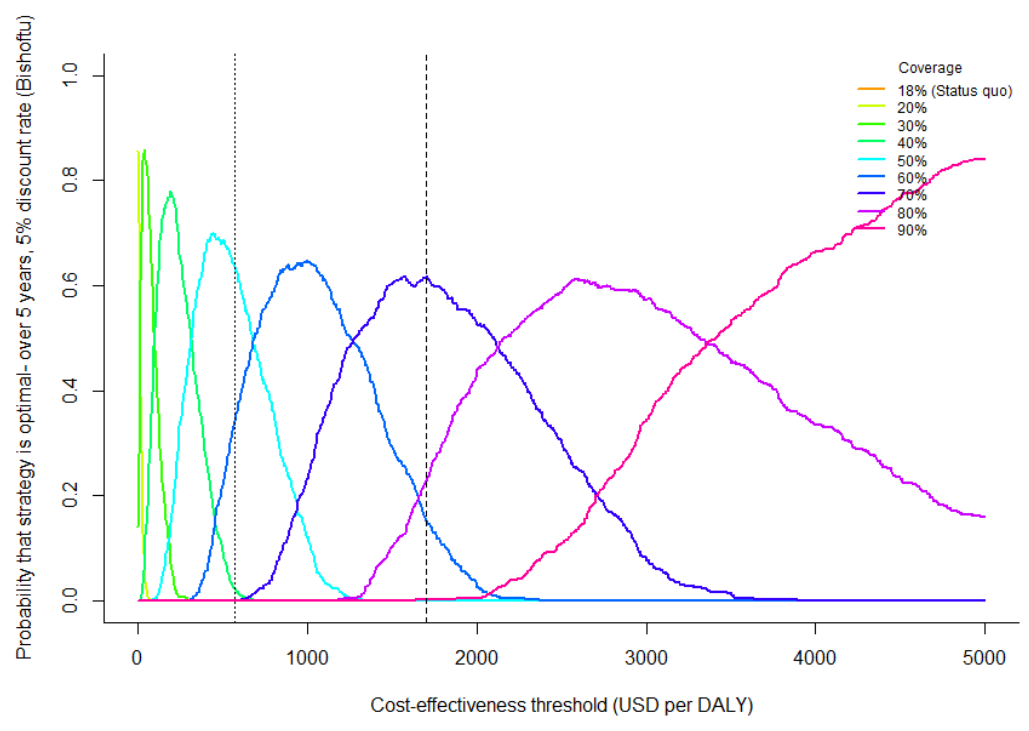

a)

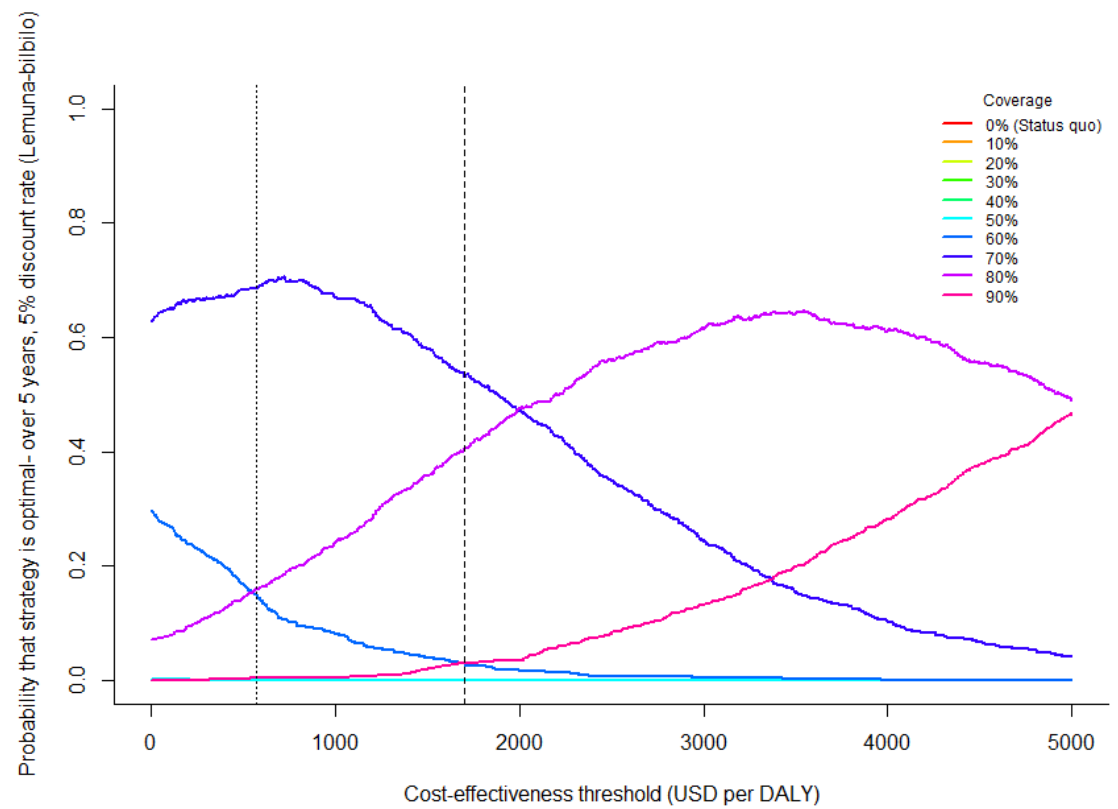

b) 


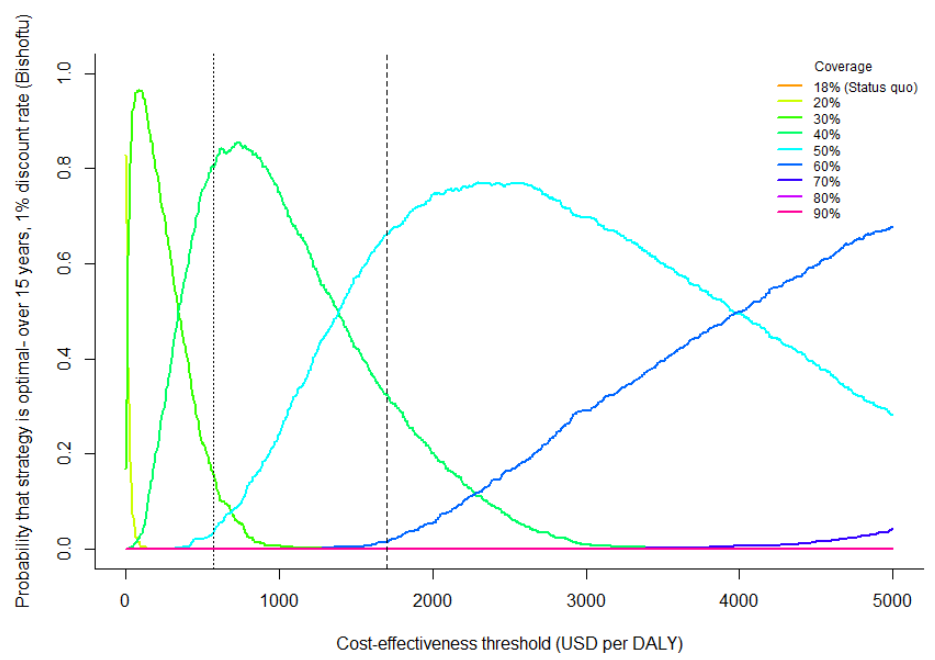

c)

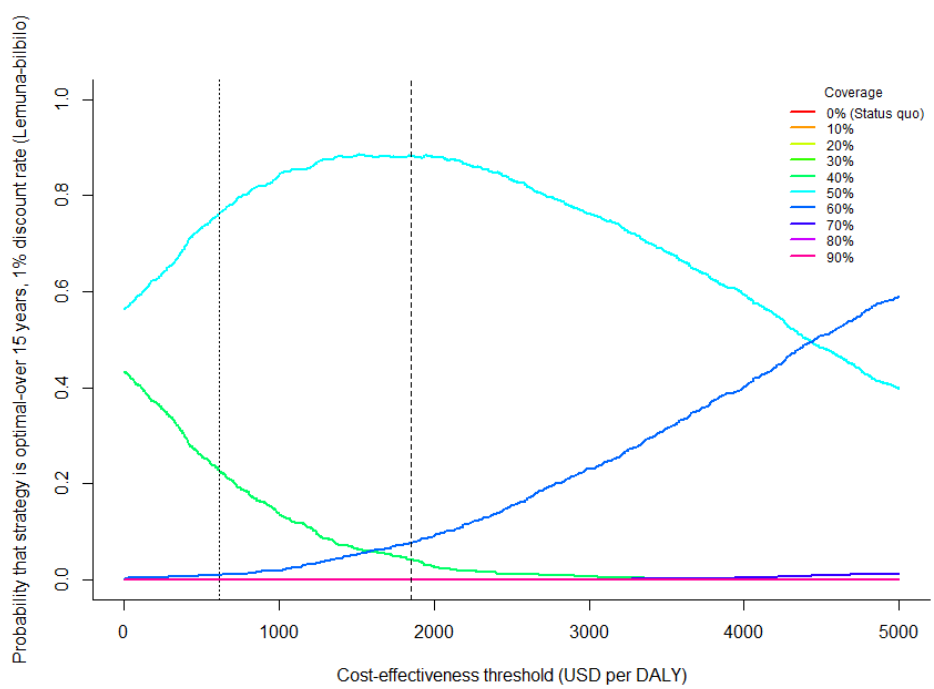

d)

Figure 3: Two-way sensitivity analysis for recommended canine vaccination coverage strategy under a) short term plan of annual vaccination campaign (5 years and $5 \%$ discount rate) a) Bishoftu, b) Lemuna-bilbilo, and long term plan of annual vaccination campaign (15 years and $1 \%$ discount rate) for c) Bishoftu and d) Lemuna-bilbilo districts. The vertical lines on the left is very cost-effective threshold (equivalent of GDP per capita) while the vertical line on the right is cost-effective threshold (equivalent of three times GDP per capita). 


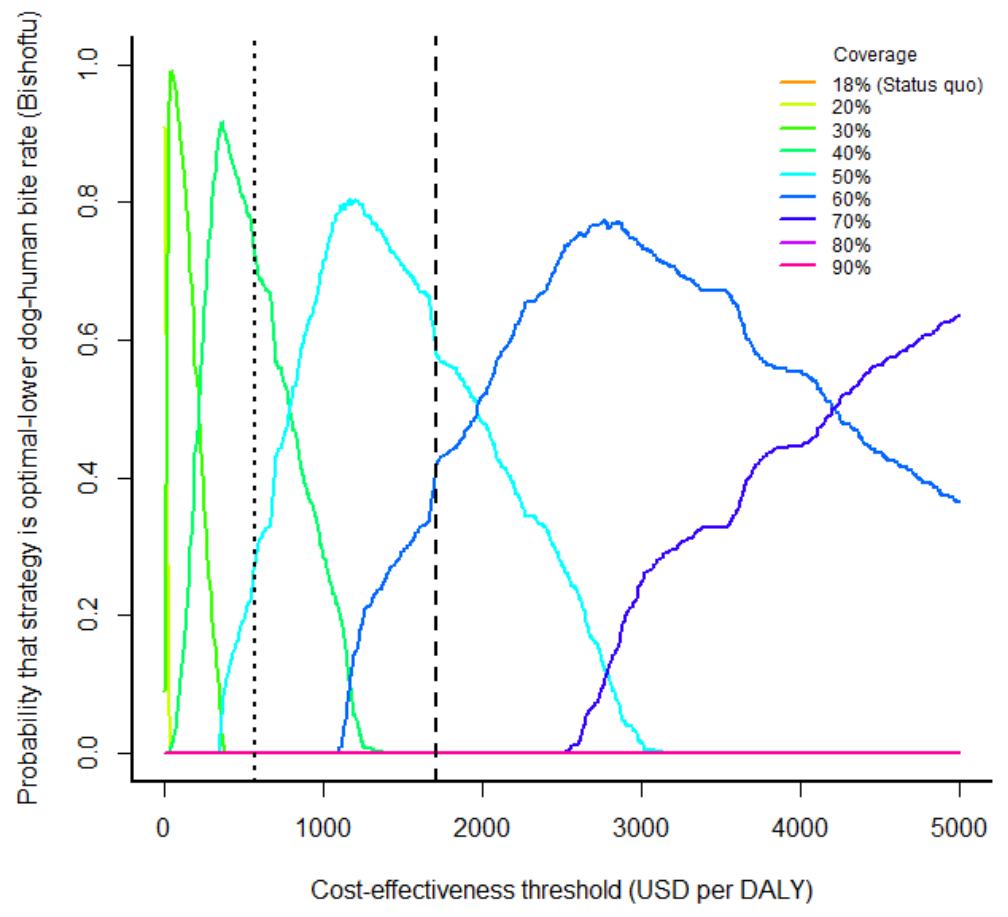

a)

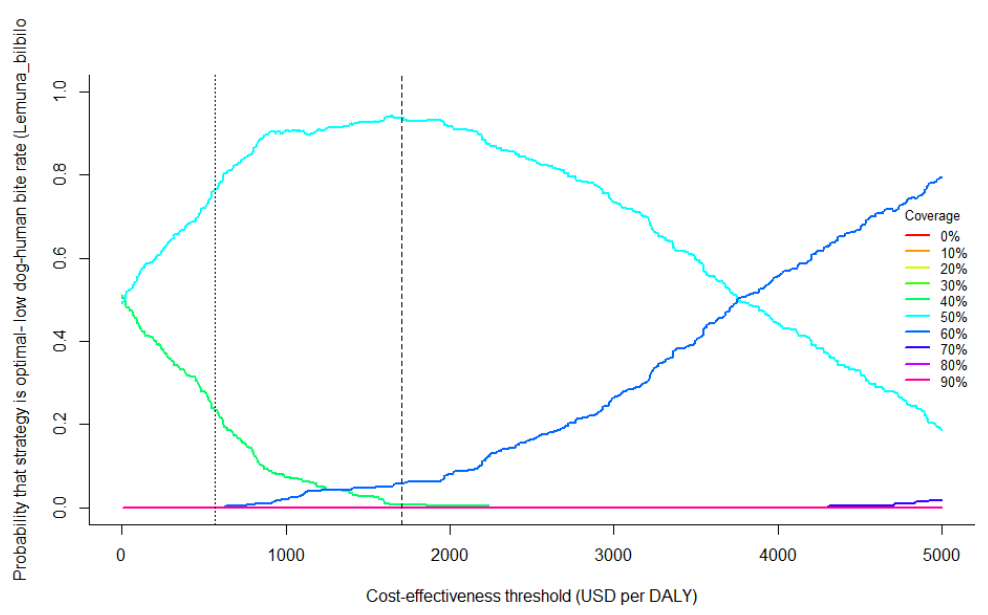

b) 


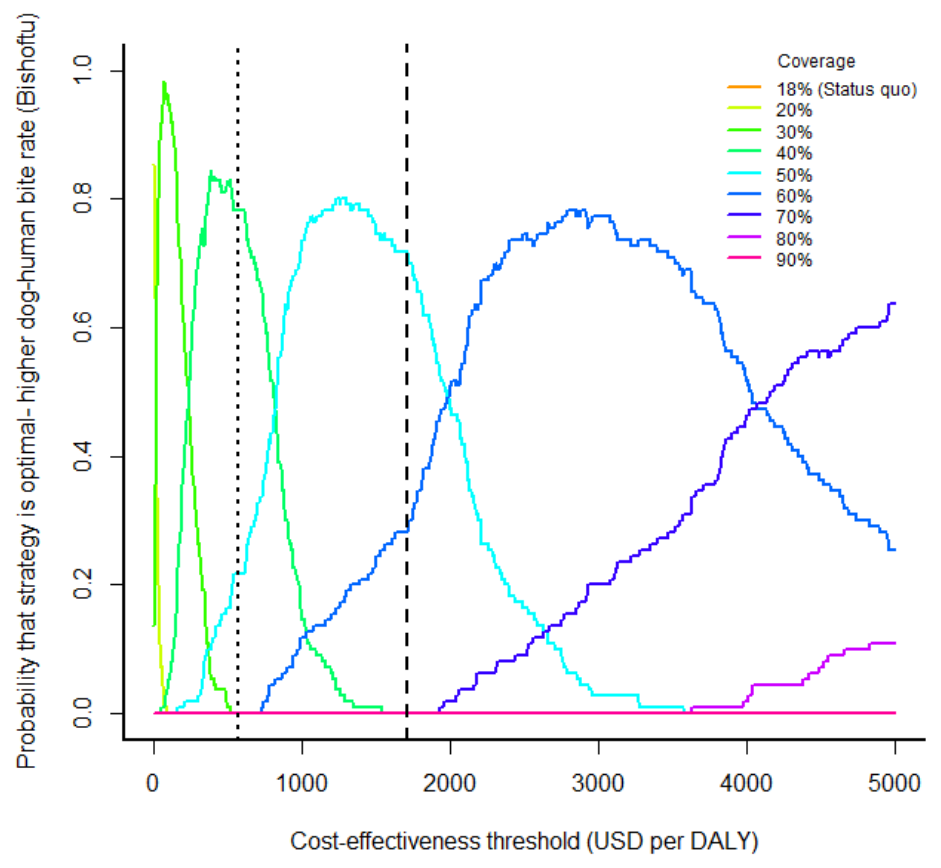

c)

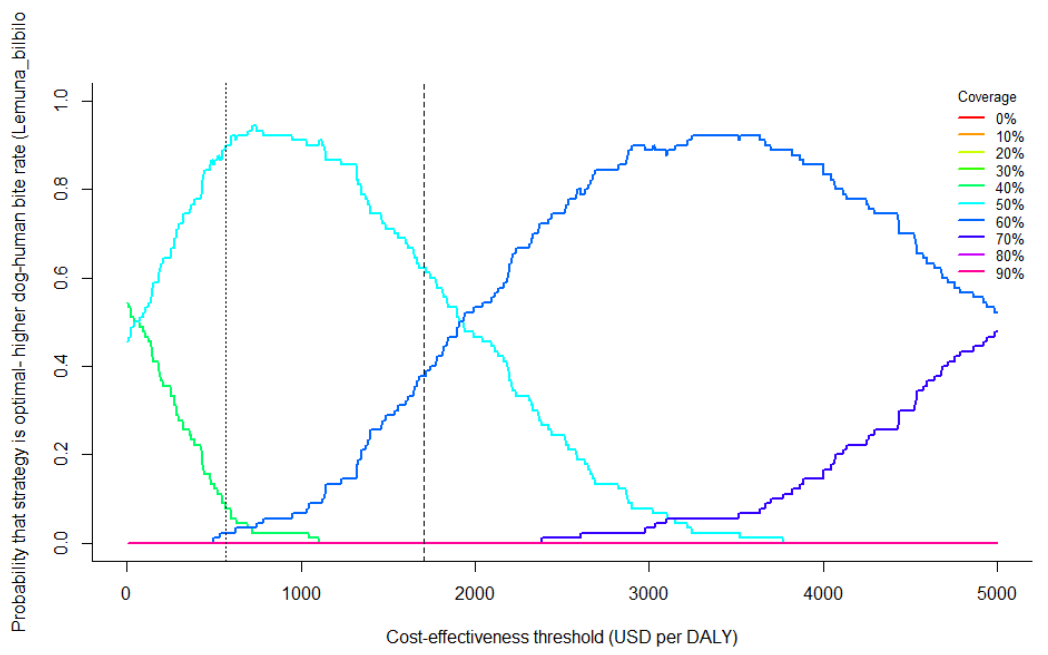

d)

Figure 4: One-way sensitivity analysis on the impact of lower (0.2) bite rate on recommended rabies vaccination coverage in a) Bishoftu b) Lemuna-bilbilo district and higher (0.99) bite rate on recommended rabies vaccination coverage in c) Bishoftu d) Lemuna-bilbilo district 


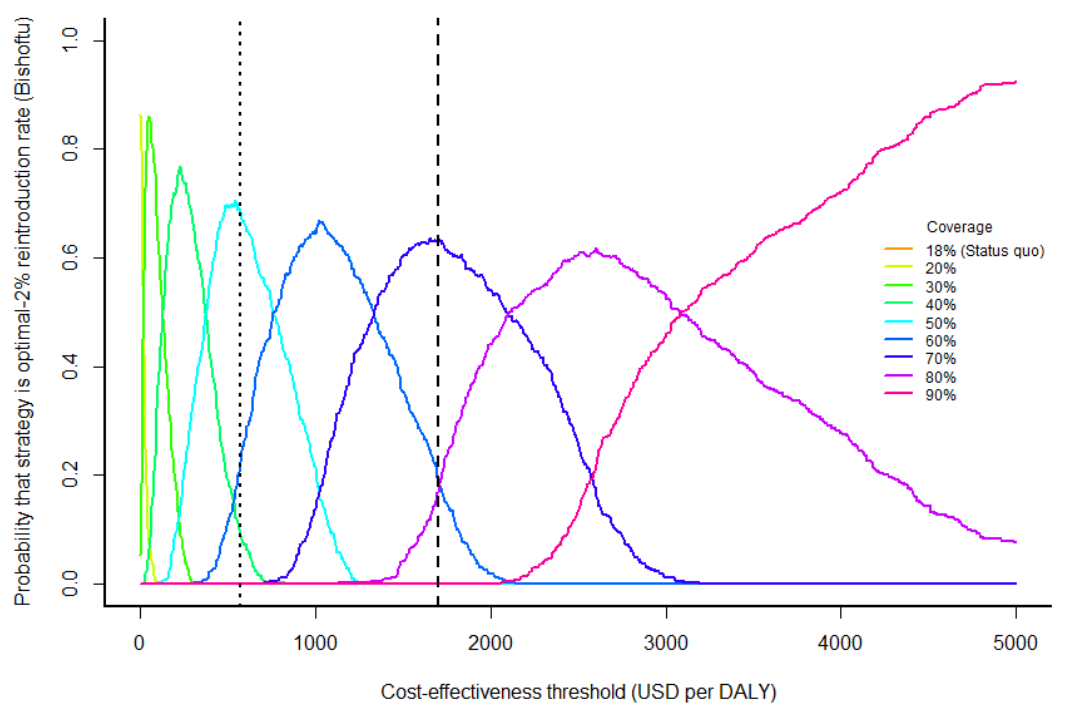

a)

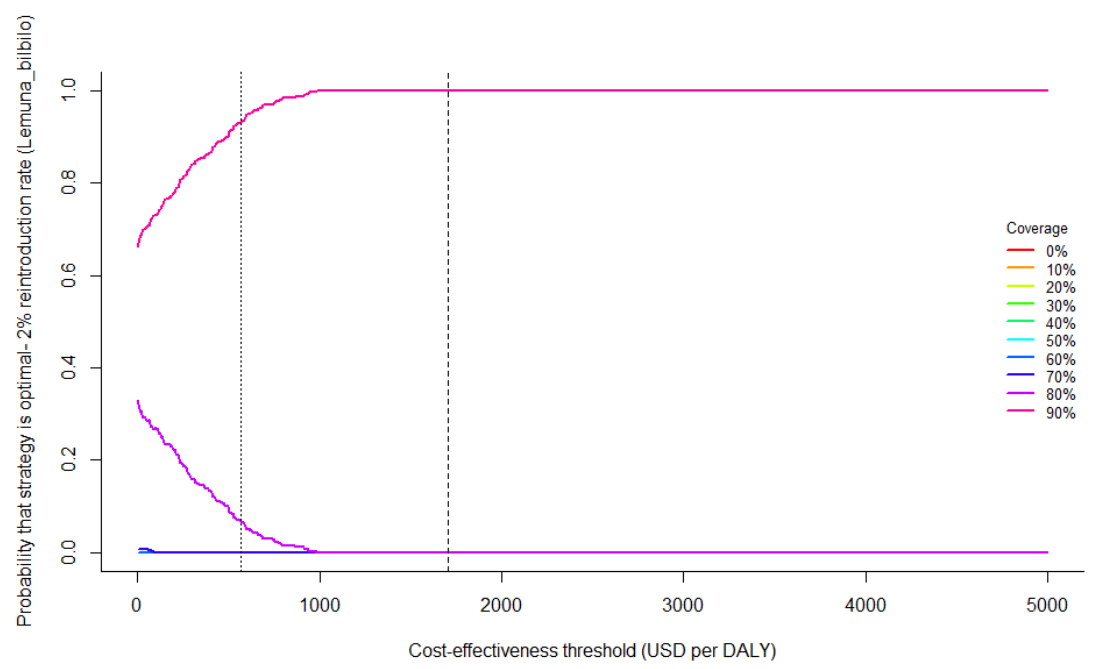

b) 


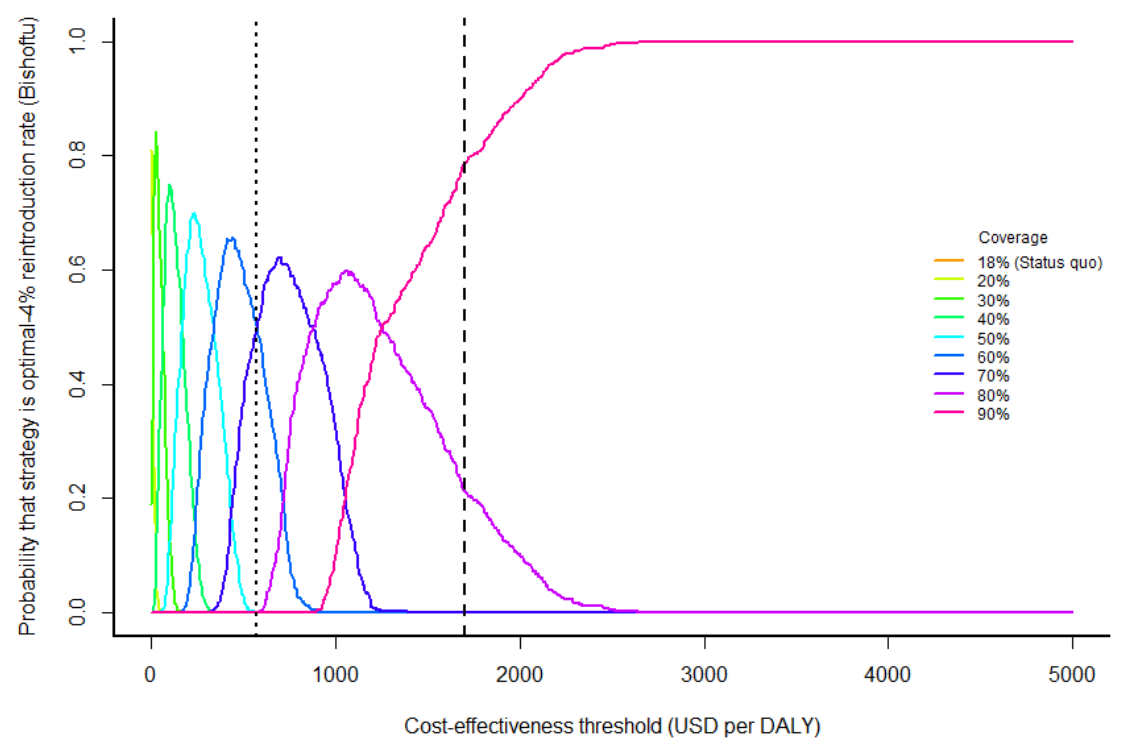

c)

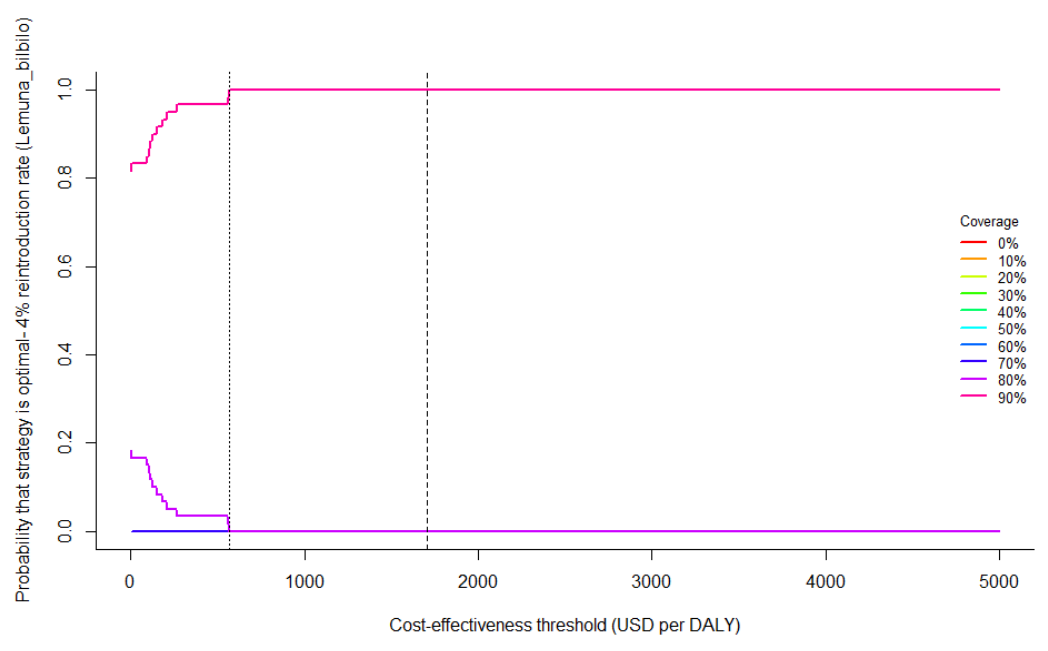

d)

Figure 5: One-way sensitivity analysis on the impact of $2 \%$ rabies re-introduction on recommended rabies vaccination coverage in a) Bishoftu b) Lemuna-bilbilo district and 4\% rabies re-introduction on recommended optimal rabies vaccination coverage in c) Bishoftu d) Lemuna-bilbilo district 


\section{References}

Beyene, T. J., Kidane, A. H., Mourits, M. C., Hogeveen, H. 2017. Public health burden and exposure costs of rabies in Ethiopia. SVEPM conference, March 29-31, 2017. Inverness, Scotland, UK.

CSA. 2013. Population Projection of Ethiopia for All Regions At Wereda Level from 2014 2017. Federal Democratic Republic of Ethiopia Central Statistical Agency,, Addis Ababa, Ethiopia.

White G. 2017. Program MARK, version 6.2, developed by Gary White, Colorado State University (http://www.phidot.org/software/mark/docs/book/). Access date 27/7/2017.

Gibson, A. D., Handel, I. G., Shervell, K., Roux, T., Mayer, D., Muyila, S., . . . Gamble, L. 2016. The Vaccination of 35,000 Dogs in 20 Working Days Using Combined Static Point and Door-to-Door Methods in Blantyre, Malawi. PLoS Neg Trop Dis, 10(7), e0004824. doi:10.1371/journal.pntd.0004824

Hampson, K., Dushoff, J., Cleaveland, S., Haydon, D. T., Kaare, M., Packer, C., Dobson, A. 2009. Transmission dynamics and prospects for the elimination of canine rabies. Plos Biology, 7(3), e53. doi:10.1371/journal.pbio.1000053

Hiby, L. R., Reece, J. F., Wright, R., Jaisinghani, R., Singh, B., Hiby, E. F. 2011. A markresight survey method to estimate the roaming dog population in three cities in Rajasthan, India. BMC Vet Res, 7(1), 46.

Jibat, T., Mourits, M. C., Hogeveen, H. 2016. Incidence and economic impact of rabies in the cattle population of Ethiopia. Prev Vet Med, 130, 67-76. doi:10.1016/j.prevetmed.2016.06.005.

Shim, E., Hampson, K., Cleaveland, S., Galvani, A. P. 2009. Evaluating the cost-effectiveness of rabies post-exposure prophylaxis: a case study in Tanzania. Vaccine, 27(51), 71677172.

Wera, E., Velthuis, A. G. J., Geong, M., Hogeveen, H. 2013. Costs of Rabies Control: An Economic Calculation Method Applied to Flores Island. PLoS One, 8(12), e83654.

WSAP. 2009. World Society for the Protection of Animals Surveying roaming dog populations: guidelines on methodology. Companion \& Working Animals Unit. World Society for the Protection of Animals, 89 Albert Embankment. London. 

Chapter 8

General Discussion 
The overall objective of this study was to support the decision-making process on the control of rabies in Ethiopia. This thesis provides quantitative evidence regarding the human health burden due to rabies as well as its economic impacts on livestock, and ultimately evaluates the cost-effectiveness of adopting canine rabies control strategies. Obtained insights inform policymakers about an optimal use of resources in reducing the impact of the disease (Nel et al., 2015; WHO, 2017).

The general objective of this study was divided into six specific sub-objectives as described in Chapter 1. Each sub-objective is addressed in detail in the Chapters 2 - 7 of this thesis. Chapter 2 and 3 systematically explores literature on canine rabies vaccination coverage and rabies datareporting in Africa. Chapter 4 presents the impact of rabies in the cattle population, while in Chapter 5 the public health burden of rabies and the costs associated with exposure to rabies are evaluated. In Chapter 6, determinants of medical treatment-seeking behaviour and of compliance to treatment regimen after being exposed to rabies are studied. Chapter 7 presents an evaluation of different canine vaccination strategies as to their cost-effectiveness. In this concluding chapter, Chapter 8 , the results from all the sub-objectives are synthesised (section 8.1), methodological approaches are discussed (section 8.2), possible policy implications of the results on rabies control are indicated (section 8.3), policy implications are provided (section 8.4) and an outlook on future research is given (section 8.5). The chapter ends with the main conclusions of this study (section 8.6).

\subsection{Synthesis of the results}

The basic idea behind this thesis was to integrate relevant aspects of epidemiology with (health) economics to produce knowledge on human as well as on livestock rabies in Ethiopia, and hence to support decision-making regarding rabies control under varying conditions of human settlement, livestock and dog densities in rural and urban settings (Figure 1.1). The most interesting results of this study are synthesised as follows.

\subsubsection{The burden of rabies}

In order to make evidence-based decisions on the control of rabies, governments need reasonably good data. Timely, accurate and complete reporting is, therefore, an essential aspect for the establishment and monitoring of disease control (Townsend et al., 2013). In Chapter 3, rabies data reported from Southern and Eastern African countries to a consortium of veterinary and public health officials called SEARG (Southern and Eastern African Rabies Group) (SEARG, 2013) and to the OIE-World Animal Health Information Database (OIE, 2014) were compared. Despite differences in entities by which data have been reported to the two organisations, reported numbers were significantly correlated. However, inconsistencies were also shown in the reported numbers of dog cases. This is possibly due to the fact that human 
and animal health authorities report separately to these organisations/consortia. Moreover, the use of ambiguous definitions of report categories set by the report receiving organisations also play a role in the inconsistencies (Nel, 2013). Yet most human health and veterinary offices in Southern and Eastern African countries seem to work solitary and are often not collaborating (Gebreyes et al., 2014; Nyatanyi et al., 2017), despite the benefits of a One-Health approach. 'One Health' is supposed to be effective in terms of cost-savings, and avoidance of duplication of efforts for diseases that are shared between animals, humans and the ecosystem (Zinsstag et al., 2005; Zinsstag et al., 2009a; Zinsstag et al., 2011; Baum et al., 2017). Rabies is one of the best examples where the One-Health approach of controlling zoonotic diseases can be applied. Whilst many African countries understand the advantages of the one-health approach and are willing to apply the principles, the reality of planning, budgeting for joint actions and defining responsibilities, particularly at the national or regional level, does not meet these ambitions (Okello et al., 2014).

Besides DALY as assessment measurement of the burden of disease in humans, we estimated the costs incurred while seeking treatment upon exposure under situations in three districts representing urban, rural highland and rural lowland, as well as the economic impacts of cattle rabies for the two rural districts (Chapters 4 and 5). In the rural highland district the mixed croplivestock production system is primarily practiced, while the rural lowland district is representative of the pastoral production system (i.e. people live mainly on keeping livestock). Based on the data that were collected, the annual average Ethiopian human death rate due to rabies was estimated at 3 deaths per 100,000 inhabitants. However, variations existed across the agro-ecology representative districts. The annual death rates and consequently DALYs were higher in rural districts (4.2 in Lemuna-bilbilo and 3 in Yabelo per 100,000 inhabitants) (Chapter 5). Conversely, the medical health-seeking behaviour and compliance to treatment regimen were lower in the rural districts. Infrastructure, specifically the distance to a health centre, was identified as one of the reasons for the disproportionate burden. Possibly other district-related factors, such as availability of other treatment options, and perception of the effectiveness of treatment could also have played a role (Chapter 6).

It is generally known that rabies also affects cattle (Okell at al., 2013), a fact that has, however, not widely been studied yet. Therefore, to make an evaluation of the burden of rabies in livestock-keeping communities, economic impact of rabies on livestock should also be accounted in order to obtain a more realistic estimation of the true burden. Livestock are a direct source of livelihood, in terms of food and cash, for the majority of Ethiopians. In addition, about 80\% of Ethiopian crop farmers use animal traction to plough their crop fields (Behnke and Metaferia, 2011) and their crop production will be affected when their oxen are diseased or lost due to rabies. Thus, a broader notion of the total disease burden by the inclusion of livestock losses would be more relevant when designing a proper and cost-effective control strategy for 
these communities than based on the human health burden alone. Chapter 4, therefore, devoted extra attention to the economic impacts of rabies in cattle and is as such innovative by providing one of the first quantitative estimations. The average annual losses due to cattle-related rabies at herd level as well as to affected households were severe. The economic burden of rabies in cattle was especially severe for farmers in a pastoral production system (rural lowland districts) (Thornton et al., 2007).

Based on this broader disease burden notion, the simulated cost-effectiveness of canine mass vaccination scenarios in a rural district, varying from a coverage of no vaccination (status quo) to a coverage of $90 \%$, showed that all tested vaccination scenarios resulted in a positive net monetary benefit (Chapter 7). Vaccination not only reduced the (economic) burden of rabies in humans but also the economic burden of rabies in cattle. Consequently, the costs of mass canine vaccination were more than compensated by a reduced burden of rabies in cattle (Chapter 7). The inclusion of benefits of canine vaccination by the reduction of the impact posed on cattle health and productivity shifted the incremental cost-effective coverage from $30 \%$ to $50 \%$, which suggests that the economic burden of rabies in cattle represents a relevant financial incentive for canine vaccination. As a result of shifting from 30\% to 50\% coverage, 39 DALYs were additionally saved on an annual base, while the economic impact decreased with 350,000 USD/year due to reduced costs on post-exposure treatment and reduced cattle-related rabies losses. Also in the urban district, canine mass vaccination had positive health impacts and savings in the long run, but these were less distinct compared to the rural district due to the absence of dependency on livestock.

Governments often use DALY or QALY estimations to rank diseases and to set priorities in health investments (GBD, 2016). Global funds also often prioritise public health-related grants following the DALY/QALY approach (Zelman et al., 2016). Rabies is an invariably fatal disease, yet it is not on the top list of 25 diseases in countries like Ethiopia where there are many diseases with a higher DALY/QALY burden (GBD, 2015). However, the burden of zoonotic diseases such as rabies encompasses not only DALYs but also productivity/income losses, treatment-related costs, and societal costs in terms of psychological and emotional anxiety. The burden also extends to its impacts on livestock health and production, and biodiversity loss due to affected wildlife, and costs of control (Hampson et al., 2015; Haydon et al., 2002; Okell et al., 2013). As such the use of only DALY/QALY measures to set priorities in health investments is not serving the overall societal interest in a best way; a broader approach is necessary and includes accounting for other relevant burden components.

\subsubsection{Evaluation of rabies control strategies}

The majority of rabies cases in humans and cattle are contracted from dogs (Chapter 4 and 5). This is a confirmation of several other studies that also reported that dogs are the major source 
of rabies in most African countries including Ethiopia (Cleaveland et al., 2003; Jemberu et al., 2013). While canine mass vaccination has been proven to be a cost-effective control option (Shim et al., 2009; Fitzpatrick et al., 2014), most African countries have failed to achieve the levels of coverage that are necessary to interrupt rabies transmission (Hampson et al., 2015; Kaare et al., 2009). One of the major reasons for this failure is the (perceived) high costs of vaccine (Lembo et al., 2010). Our findings in Chapter 2 showed that dog rabies vaccination coverage following ''free-of-charge”' vaccination schemes was higher (on average 68\%) than the coverage rate under '’owner-charged'” dog rabies vaccination schemes (on average 18\%).

Similarly, in Ethiopia, canine vaccination coverage is approximately $18 \%$ in urban districts located close to the capital city. Coverages are lower in city districts further away and in rural districts. While the impacts of rabies on human health and livelihoods are higher in rural districts (Chapter 5), canine vaccination against rabies is almost non-existent in rural districts (Ali et al., 2010; Kabeta et al., 2015). Ethiopia has no compulsory dog vaccination regulation and dog owners do not have a personal incentive to have their dogs vaccinated as it only offers limited reduction on the risk of obtaining rabies. Vaccination of one's own dog does not guarantee protection against exposure with rabies by other dogs. Another reason for the low coverage under the "'owner-charged" vaccination scheme could be the inaccessibility of private or public vaccinators on demand. In the case of 'free-of-charge'" vaccination schemes, campaign organisers have a door-to-door campaign or create a temporary central point for vaccinations (Cleaveland et al., 2003; Kebede et al., 2014).

Assuming an annual cost-effective canine mass vaccination campaign with a coverage of $50 \%$, the total investment for the whole of Ethiopia is estimated to be 18.2 million USD/year (Chapter 7). Although the benefits of such a campaign, in terms of improved cattle health and reduced costs of post-exposure treatments, are higher than the costs, the costs and benefits are not equally distributed. The costs for the vaccination campaign requires public resources, while the benefits are specifically for cattle owners and rabies-exposed individuals. Moreover, an investment of 18.2 million USD is a large investment for the Ethiopian government to allocate to rabies control compared to the amount of budget allocated to the health sector in general. Following the global target to eliminate dog-mediated human rabies by 2030, there is pressure for international donors and charitable organisations to support governments in coordinated actions (OIE, 2011; WHO, 2017). Currently no global affirmative action has been taken. Moreover, a sustained, multiple-year intervention programme and hence investment is needed as a single investment will not be enough to control rabies. This thesis showed that a probability of re-introduction as low as $2 \%$ can already significantly hamper the progress of rabies control (Chapter 7). 
As rabies control leads to savings both in public health as well as in veterinary health, a joint approach, also referred to as "separable costs-remaining benefits" by public health and veterinary sectors, to allocate the expenditures to both sectors proportional to the benefits gained by both sectors, can be used (Gittinger, 1972). Such a proportional allocation of resources was also simulated for Rift Valley Fever control in Kenya (Kimani et al., 2016) and Brucellosis control in Mongolia (Roth et al., 2003). A more continuous rabies control programme was demonstrated in Bohol (Indonesia) through legalising the control framework (i.e. compulsory dog registration to establish responsible pet ownership and accountability in combination with mass vaccination to establish dog-herd immunity), mobilising local resources and involving the local community (Lapiz et al., 2012). Alternatively, governments can apply for loan options such as the development impact fund (Welburn et al., 2016). This approach is a form of social impact bond, whereby initial costs of disease control are supported by private investors and repaid by donors and governments once agreed outcomes are achieved. These schemes were demonstrated to work for the control of sleeping sickness in Uganda (Fyfe et al., 2017; Welburn et al., 2016). Given that rabies has a readily available effective vaccine but requiring a relatively large initial investment, rabies control would be a perfect candidate for such financing in countries like Ethiopia.

\subsection{Methodological approaches}

The overall approach of this study was interdisciplinary, involving methodologies from different scientific fields in the range of epidemiology and (health) economics. To address the different specific objectives of this study, methodologies such as systematic review and metaanalysis (Chapter 2 and 3), deterministic economic modelling (Chapter 4), disease burden analysis (DALYs and cost of illness analyses) (Chapter 5), multivariable logistic regression modelling (Chapter 6) and integrated bioeconomic modelling (Chapter 7) were applied. In this section, emphasis will be given to some interesting methodological aspects from the thesis: the main approaches of human and livestock rabies data collection and bioeconomic modelling techniques used in this study with respect to their competitive advantages within the context of this study.

Registered rabies cases, both by veterinary and human authorities, are underreported in many African and Asian countries. Consequently, officially registered data on rabies underestimate the true burden of the disease (Cleaveland et al., 2002; Tenzin et al., 2011; Taylor et al., 2017). In Ethiopia, hardly any incentive mechanisms exist for public reporting of health-related incidences, unless people report while seeking medical treatment in health centres. The healthseeking practices are shown to vary between rural and urban areas (Chapter 6). The wide presence of traditional/spiritual healers in especially the rural areas (Kabeta et al., 2015), which neither have any working connection with medical health centres nor share reports, if any, might also contribute to the underreporting. Therefore, to prevent underreporting, in this study, a 
survey-based case investigation was utilised to fill the gap of underreporting. Despite the difficulty of assessing the accuracy of such an approach, we reported more cases than those recorded at the health centres. Further improvement towards reliable data could only have been made through a detailed community-based survey which is very resource intensive.

In this study, those human exposure cases that were not registered at health centres were contacted and investigated using the contact-tracing method which can be seen as a 'snowballing', approach. The seed cases in this method were received from health centre records and through these seed cases other cases, not known to the health centres, could be identified. This method of data collection was also used in various other studies for a range of diseases in countries where disease cases are not fully registered (e.g., Riley et al., 2003; Hampson et al., 2009; Smith and Aerts, 2014). The contact-tracing method we used in this study gave the best possible opportunity to access unregistered rabies exposures and deaths. It was, however, not possible to confirm all deaths.

In this research, three districts were purposefully selected and evaluated. Each district represented one of the three different agro-ecological systems of Ethiopia. Given that the data we collected covered the incidence of rabies in each district for only one year, there could be temporal variation in rabies incidence between years. The public health burden estimation in life years lost is also susceptible to small changes in the number of data entries. In addition, there is a possibility of spatial heterogeneity of the distribution of rabies incidence within a specific setting, i.e., urban and rural districts. Better national estimates could be produced on data collected over more years from multiple districts within each specific setting. However, given the resource constraints we had in our research, the results of this study give the best possible estimate of the rabies situation in Ethiopia, although care should be taken when extrapolating the burden estimation to the national level.

Until now, no reliable quantified information on the health and economic impacts of rabies in livestock has been available for Ethiopia. Such quantification has been a challenge due to lack of data. The use of a participatory approach by involving the farming community to generate livestock health data in this thesis, has shown to be useful to obtain the best possible estimate of the health and economic impact of rabies on cattle (Jost et al., 2007). The fact that we had to rely on the ability of the farming community to recognise rabies did not compromise the estimate we made, because also in livestock rabies has very typical clinical signs. The response bias was minimised by not informing the livestock owners and local facilitators that the main disease of interest was rabies. Also, each reported exposure incident by suspected dogs was examined with the standard six-criteria method which was reported to have a high accuracy of 96\% (Tepsumethanon et al., 2005). In case of rabies in cattle, the clinical signs manifested by 
rabid cattle was compared to the clinical signs mentioned in clinical text book (Kahn et al., 2010).

There are various approaches of deriving input parameters for transmission models to simulate the effectiveness of control strategies. In this study a data-driven approach was used to generate input parameters, which are generic and potentially possible to be customised based on specific situations of human and canine density. Such a data-driven approach requires extensive data and advanced Bayesian expertise. Most of the time, researchers depend on literature and expert opinions to define the required transmission input parameters due to a lack of the required data (Wera et al., 2016). However, cost-effectiveness analyses based on assumptions estimating district-specific transmission parameters would not be feasible to serve context-specific decision support. In situations where there could be different motivations of controlling rabies such as presence of susceptible livestock, wildlife or tourism motives, and varying human and canine densities, situation-specific cost-effectiveness analysis requires attention. In such circumstances, specific transmission parameter values may need to be estimated from observed data (Woolhouse, 2011). In this study the transmission parameter inputs were derived from a data-driven rabies transmission model to fit the human rabies and canine demography data from the aforementioned two districts. Parameters were generated based on a Bayesian Monte-Carlo Markov chain (MCMC) approach by updating prior parameters to fit the observed data on human rabies incidence and canine population size, while accounting for uncertainty around the mean. .

A proper cost-effectiveness analysis of disease-control options requires well-designed epidemiological and economic models with realistic parameter inputs that reasonably predict the health and economic outcomes under each option considered. In this study we adjusted an existing SEIVR compartmental rabies transmission model (Fitzpatrick et al., 2014) for two typical Ethiopian urban and rural highland districts, namely Bishoftu and Lemuna-bilbilo. The advantage of such a model is the stochasticity and the closer-to-reality simulation which incorporate the dynamics of rabies transmission and demography within the domestic dog population, and generates useful insights into a range of outcomes arising from variations and uncertainty in input parameters. Compared to a deterministic transmission model which is often based on literature and expert opinion, the model used in this study is tailor-made and capable of providing context-specific insights. Hence, it helps policy makers to understand the specific differences on the epidemiology of the disease across districts. Such transmission models have been used to evaluate cost-effectiveness of canine mass vaccination strategies in Chad (Zinsstag et al., 2009a), Tanzania (Fitzpatrick et al., 2014) and India (Fitzpatrick et al., 2016). As such, a deterministic modelling approach is good for a first exploration on vaccination strategies, while the applied stochastic modelling approach is preferred for fine tuning to gather detailed and context specific information. 


\subsection{Policy Implications}

In many developing countries, including Ethiopia, there is neither a working policy nor enforced regulation on rabies control (EHNRI, 2012). In Ethiopia, the country is divided into nine regions and two city administrations, each consisting of several districts. As regions in Ethiopia are autonomous, they are meant to be responsible for the control of rabies within their political boundaries (Coetzer et al., 2016). In all regions, canine vaccination is voluntarilybased, dog-owner charged and the vaccination coverage varies from $18 \%$ in urban areas to nonexistent in rural areas. Inhabitants of urban and rural areas in Ethiopia form approximately $20 \%$ and $80 \%$ of the total population, respectively (CSA, 2013). In Africa, in general, owner-charged canine vaccination schemes have been shown to have far lower coverage than free or sponsored mass vaccination campaigns (Chapter 2).

Based on our findings, 50\% canine rabies vaccination coverage is a cost-effective strategy to control rabies. However, given the situation in Africa in general and in Ethiopia specifically, owner-charged dog vaccination is expected to result in a far lower coverage. This would require government involvement for covering the costs of vaccination. The Ethiopian government allocated about $7-11 \%$ of the total fiscal budget to the health sector (WHO, 2009) which equals 388 million USD for the year 2009. Allocating an estimated 18 million USD annually for canine vaccination (Chapter 7) alone, could be challenging, rabies being not the priority of the health sector, which suggest that there have to be other means of financing dog vaccination.

In urban districts there are more free-roaming dogs (owned and/or without owners) compared to rural areas. Systematic culling of unowned dogs and setting up dog-ownership regulations could be used to enforce legal responsibility. Furthermore, various options could be used to bridge the gap of limited financial resources. A partial dog-owner contribution could be applied as demonstrated in Asia (Miranda et al., 2015). In rural livestock-owning communities, the impact of rabies on cattle health and productivity in addition to its public health impacts could be viewed as an incentive. Awareness creation stating that dog vaccination is cheaper than the related loss in cattle could convince farmers to participate in either owner-charged or cofunding vaccination campaigns.

Despite the fact that the benefits of rabies control outweighs the costs of control, the beneficiaries and those who bear the costs (dog owners) are not necessarily the same. This indicates rabies control to be a public good. As indicated in section 8.1, governments could follow financing strategies such as joint financing including the "separable costs-remaining benefits" method of cost sharing (Gittinger, 1972) between the veterinary and public health sectors. Other potential synergistic funding options for vaccination campaigns could include a loan through development-impact funding, where investments are paid back over several years once savings are noticed as a result of benefits from disease control (Welburn et al., 2016). 
Collaboration between public and animal health authorities in terms of expertise and resources could also be a more sustainable option. An USAID (United States of Agency for International Development)-funded project in Ethiopia recently recommended the establishment of a OneHealth focused zoonotic disease unit, comprising of officers from the Ministry of Health (represented by the Ethiopian Public Health Institute) and the Ministry of Livestock and Fisheries (Pieracci et al., 2016). Establishment of such units at different administrative levels, including practicing veterinarians and medical doctors would improve communication about specific risks, and could contribute to a practical One-Health-oriented cooperation. Such collaboration between medical doctors and veterinarians can also avoid unnecessary expenses due to post-exposure prophylaxis costs in the case that biting dogs are not suspected of rabies.

Re-introduction of rabies is one of the challenges in controlling rabies, as is shown in Chapter 7. In this study we simulated the cost-effectiveness analysis with 2-4\% annual re-introduction and found cost-effective vaccination coverage to be higher than the scenario without reintroduction risk. This implies the need for a concerted collaboration with municipalities to ensure responsible dog ownership and movement control, and with wildlife authorities for regular surveillance at domestic-wildlife interfaces to prevent re-introduction.

Besides reducing the risk of being bitten by a rabid dog through dog rabies control programmes, the health burden of rabies can also be alleviated by improving the health-seeking behaviour of people that have been bitten by a suspected rabid dog. Chapter 4 showed that the likelihood of visiting a health centre after having been exposed to rabies and the likelihood to receive sufficient doses of treatment are heavily influenced by the distance from the victim's village to the health centres delivering post-exposure prophylaxis (PEP). This calls for availing health centres delivering PEP within short distances. In urban areas, as most private health centres do not deliver post-exposure prophylaxis services, establishing a system that involves all public and private health centres to participate in delivering PEP service could alleviate the problem of availability in the short run. In rural areas, there are a few private public health centres while the public health centres are sparsely distributed, which means more health centres should be established.

In this study we found that the rabies' health and economic burden (Chapter 5), the medical treatment-seeking behaviour (Chapter 6), and the benefits of control (Chapters 7) differ across rural and urban districts. Therefore, decision makers need to account for these differences between districts in attempts to prioritise and control the disease. 


\subsection{Research gaps and future perspectives}

This thesis provides relevant insights on the burden and cost-effectiveness of control strategies to support decision-making to control rabies in Ethiopia. Although we have produced most important information on the burden of rabies and its control strategies, there are, however, still knowledge gaps that hinder policymakers to take the best possible informed decisions. Below we describe some recommended areas of research in the field of epidemiology and economics of the disease, that need to be addressed to produce more evidence to provide for a wellinformed decision-support process in the control of rabies.

In Chapter 2, we showed that, in Africa, owner-charged dog vaccination schemes demonstrated low coverage and we forwarded the policy implication to devise a means of financing dog vaccination. The study we carried out on the consistency of rabies data reported by African public and animal health authorities showed that these were inconsistent. Cross-sectoral collaborations such as the One-Health approach are advocated as an effective and cost-saving means of managing such diseases of public and animal health interest. Operationalising such a cross-sectoral agenda is challenging in most countries (Okello et al., 2014). However, some countries, Kenya for instance, have successfully established a zoonotic disease unit (Mbabu et al., 2014). Apart from rabies, in Ethiopia some of the top-listed diseases in terms of health burden like diarrhoeal diseases are partly zoonotic (GBD, 2015), indicating a broader benefit for operationalising an One Health approach than from rabies alone.

In our study on the health-seeking behaviour of victims of a rabid dog bite, we considered potential determinant factors affecting likelihoods of health-seeking behaviours and compliance with treatment regimen. Similar studies in other countries show that the level of knowledge on the disease and the availability of PEP at health centres varied, based on level of infrastructure and the agro-ecology (Sambo et al., 2013). Hence, further research on the impact of districtspecific situations can be explored using field experiments and/or behavioural-framework studies based on theories such as the theory of planned behaviour or the health belief framework.

In situations where ongoing disease interventions and related information are lacking, simulation models are essential tools (Willeberg et al., 2011). In the simulation models we built in Chapter 7, assumptions were used for some input parameters. However, important input assumptions which could impact the final outputs can be validated and improved, if necessary, through incorporating field effectiveness of rabies vaccination under district-specific conditions. For instance, the level of protective immunity development varies based on the health and nutritional status of dogs (Hampson et al., 2009). The specific turnover rate of dogs also has an influence on the optimal frequency of vaccination. The inclusion of livestock in the cost-effectiveness model of rabies control in a rural area has shifted the cost-effective 
vaccination coverage from 30 to $50 \%$ which means that inclusion of livestock aspect of rabies burden could significantly contribute to the ratio. This might also be the case if wildlife/tourism/conservation, and burden of rabies in other livestock species are added.

\subsection{Main conclusions}

Based on the results described in this thesis, the following main conclusions are drawn:

- In Africa, canine vaccination coverage against rabies under owner-charged scheme (18\%) is far below the free-of-charge scheme (68\%) (Chapter 2). On the other hand, most dogs in Africa are owned and accessible for vaccination against rabies if campaigns are performed at reduced costs or free-of-charge.

- Based on the available data from African regional (SEARG) and international (OIE) organisations, there is no evidence of an increasing or decreasing trend of rabies occurrence in Southern and Eastern African countries (Chapter 3).

- On average household level, rabies in cattle causes comparable economic losses (49-52 USD/year) in mixed crop-livestock and in pastoral farming systems. Losses in pastoral households (477 USD/year) are significantly higher than in mixed crop-livestock affected households (228 USD/year) signifying a serious impact as they mainly rely on their livestock for livelihoods (Chapter 4).

- Based on data collected from three representative districts in Ethiopia, the highest human rabies exposure rate is in urban district (135/100,000 inhabitants), while the health burdens are higher in rural districts (3-4 deaths per 100,000 inhabitants) than in urban (1 death per 100,000 inhabitants) districts (Chapter 5).

- Annually, rabies causes the death of about 3,000 people and leads to treatment-related expenditures of about 2 million USD nationally (Chapter 5).

- The likelihood of seeking medical care following rabies exposure is higher in people bitten by potentially rabid dogs of unknown ownership, with severe bites, and who are living in a short distance from the nearest health centre (Chapter 6).

- The shorter the distance to the nearest health centre and the higher the income, the more likely bite victims comply with the treatment regimen (Chapter 6).

- Annual canine mass vaccination at 50\% coverage is the most cost-effective strategy to control canine rabies in urban districts as well as rural districts of Ethiopia (Chapter 7). 


\subsection{References}

Addis, G., Abebe, D., Genebo, T., and Urga, K. 2002. Perceptions and practices of modern and traditional health practitioners about traditional medicine in Shirka District, Arsi Zone, Ethiopia. Ethiopian Journal of Health Development, 16(1), 19-23.

Ali, A., Mengistu, F., Hussen, K., Getahun, G., Deressa, A., Yimer, E., and Tafese, K. 2010. Overview of Rabies in and around Addis Ababa, in Animals Examined in EHNRI Zoonoses Laboratory Between, 2003 and 2009. Ethiopian Veterinary Journal, 14(2), 91-101.

Baum, S. E., Machalaba, C., Daszak, P., Salerno, R. H., and Karesh, W. B. 2017. Evaluating one health: Are we demonstrating effectiveness? One Health, 3, 5-10. doi:10.1016/j.onehlt.2016.10.004.

Behnke, R.,and Metaferia, F., 2011. The contribution of livestock to the Ethiopian economyPart II. IGAD LPI Working Paper No. 02 - 11, Addis Ababa, Ethiopia.

Cleaveland, S., Fevre, E. M., Kaare, M., and Coleman, P. G. 2002. Estimating human rabies mortality in the united republic of Tanzania from dog bite injuries. Bull World Health Org, 80.

Cleaveland, S., Kaare, M., Tiringa, P., Mlengeya, T., and Barrat, J. 2003. A dog rabies vaccination campaign in rural Africa: impact on the incidence of dog rabies and human dog-bite injuries. Vaccine, 21(17), 1965-1973. doi:http://dx.doi.org/10.1016/S0264410X(02)00778-8

Coetzer, A., Kidane, A. H., Bekele, M., Hundera, A. D., Pieracci, E. G., Shiferaw, M. L., . . . Nel, L. H. 2016. The SARE tool for rabies control: Current experience in Ethiopia. Antiviral Research, 135, 74-80. doi:10.1016/j.antiviral.2016.09.011

CSA. 2013. Population Projection of Ethiopia for All Regions At Wereda Level from 2014 2017. Federal Democratic Republic of Ethiopia Central Statistical Agency,, Addis Ababa, Ethiopia.

EHNRI. 2012. Proceding of the national workshop on Rabies prevention and control in Ethiopia, Oct 18-19, 2012, Addis ababa.

Fitzpatrick, M. C., Hampson, K., Cleaveland, S., Mzimbiri, I., Lankester, F., Lembo, T., ... . Galvani, A. P. 2014. Cost-effectiveness of canine vaccination to prevent human rabies in rural Tanzania. Annals of Internal Medicine, 160(2), 91-100.

Fitzpatrick, M. C., Shah, H. A., Pandey, A., Bilinski, A. M., Kakkar, M., Clark, A. D., . . . Galvani, A. P. 2016. One Health approach to cost-effective rabies control in India. Proceedings of the National Academy of Sciences, 113(51), 14574-14581.

Fyfe, J., Picozzi, K., Waiswa, C., Bardosh, K. L., and Welburn, S. C. 2017. Impact of mass chemotherapy in domestic livestock for control of zoonotic T. b. rhodesiense human African trypanosomiasis in Eastern Uganda. Acta Tropica, 165, 216-229.

GBD.2015.http://www.healthdata.org/sites/default/files/files/country_profiles/GBD/ihme_gbd _country_report_ethiopia.pdf. access date June 21, 2017. 
GBD. 2016. Global, regional, and national disability-adjusted life-years (DALYs) for 315 diseases and injuries and healthy life expectancy (HALE), 1990-2015: a systematic analysis for the Global Burden of Disease Study 2015. The Lancet, 388(10053), 16031658. doi:http://dx.doi.org/10.1016/S0140-6736(16)31460-X

Gebreyes, W. A., Dupouy-Camet, J., Newport, M. J., Oliveira, C. J., Schlesinger, L. S., Saif, Y. M., . . . Wittum, T. 2014. The global one health paradigm: challenges and opportunities for tackling infectious diseases at the human, animal, and environment interface in low-resource settings. PLoS Negl Trop Dis, 8(11), e3257.

Gittinger, J. 1972. Economic analysis of agricultural projects. The Economic Institute, International Bank for Reconstruction of Development: The Johns Hopkins University Press, Baltimore-London.

Hampson, K., Coudeville, L., Lembo, T., Sambo, M., Kieffer, A., Attlan, M., . . . Global Alliance for Rabies Control Partners for Rabies, P. 2015. Correction: Estimating the global burden of endemic canine rabies. PLoS Negl Trop Dis, 9(5), e0003786. doi:10.1371/journal.pntd.0003786

Hampson, K., Dushoff, J., Cleaveland, S., Haydon, D. T., Kaare, M., Packer, C., and Dobson, A. 2009. Transmission dynamics and prospects for the elimination of canine rabies. Plos Biology, 7(3), e1000053.

Haydon, D., Laurenson, M., and Sillero-Zubiri, C. 2002. Integrating epidemiology into population viability analysis: managing the risk posed by rabies and canine distemper to the Ethiopian wolf. Conservation Biology, 16(5), 1372-1385.

Jemberu, W. T., Molla, W., Almaw, G., and Alemu, S. 2013. Incidence of Rabies in Humans and Domestic Animals and People's Awareness in North Gondar Zone, Ethiopia. Plos Neglected Tropical Diseases, 7(5), e2216. doi:10.1371/journal.pntd.0002216

Jost, C., Mariner, J., Roeder, P., Sawitri, E., and Macgregor-Skinner, G. 2007. Participatory epidemiology in disease surveillance and research. Revue Scientifique Et TechniqueOffice International Des Epizooties, 26(3), 537.

Kaare, M., Lembo, T., Hampson, K., Ernest, E., Estes, A., Mentzel, C., and Cleaveland, S. 2009. Rabies control in rural Africa: evaluating strategies for effective domestic dog vaccination. Vaccine, 27(1), 152-160.

Kabeta, T., Deresa, B., Tigre, W., Ward, M. P., and Mor, S. M. 2015. Knowledge, attitudes and practices of animal bite victims attending an anti-rabies health center in Jimma Town, Ethiopia. PLoS Negl Trop Dis, 9(6), e0003867.

Kahn, C., Line, S., and Co, M. 2010) The Merck Veterinary Manual. A handbook of diagnosis, therapy, and disease prevention and control for the veterinarian. Eds: . Inc., New Jersey, USA, 905-908.

Kebede, H., Melaku, A., and Kebede, E. 2014. Constraints in animal health service delivery and sustainable improvement alternatives in North Gondar, Ethiopia: original research. Onderstepoort Journal of Veterinary Research, 81(1), 1-10. 
Kimani, T., Schelling, E., Bett, B., Ngigi, M., Randolph, T., and Fuhrimann, S. 2016. Public Health Benefits from Livestock Rift Valley Fever Control: A Simulation of Two Epidemics in Kenya. Ecohealth, 13(4), 729-742. doi:10.1007/s10393-016-1192-y

Lapiz, S. M. D., Miranda, M. E. G., Garcia, R. G., Daguro, L. I., Paman, M. D., Madrinan, F. P., . . . Briggs, D. J. 2012. Implementation of an Intersectoral Program to Eliminate Human and Canine Rabies: The Bohol Rabies Prevention and Elimination Project. Plos Neglected Tropical Diseases, 6(12), e1891.

Lembo, T., Hampson, K., Kaare, M. T., Ernest, E., Knobel, D., Kazwala, R. R., . . Cleaveland, S. 2010. The feasibility of canine rabies elimination in Africa: dispelling doubts with data. PLoS Negl Trop Dis, 4(2), e626. doi:10.1371/journal.pntd.0000626

Mbabu, M., Njeru, I., File, S., Osoro, E., Kiambi, S., Bitek, A., . . . Gogstad, E. 2014. Establishing a one health office in Kenya. The Pan African medical journal, 19.

Miranda, L., Miranda, M., Hatch, B., Deray, R., Shwiff, S., Roces, M., and Rupprecht, C. 2015. Towards canine rabies elimination in Cebu, Philippines: assessment of health economic data. Transboundary and Emerging Diseases.

Nel, L. H. 2013. Discrepancies in data reporting for rabies, Africa. Emerging Infectious Diseases, 19(4), 529-533. doi:10.3201/eid1904.120185

Nel, L. H., Taylor, L. H., Balaram, D., and Doyle, K. A. 2015. Global partnerships are critical to advance the control of Neglected Zoonotic Diseases: The case of the Global Alliance for Rabies Control. Acta Tropica.

Nyatanyi, T., Wilkes, M., McDermott, H., Nzietchueng, S., Gafarasi, I., Mudakikwa, A., . . . Mupfasoni, D. 2017. Implementing One Health as an integrated approach to health in Rwanda. BMJ Global Health, 2(1), e000121.

OIE. 2011. World Organization for Animal Health. Rabies Control-Towards Sustainable Prevention at the Source." Compendium of the OIE Global Conference on Rabies Control. September 7-9, 2011. Incheon-Seoul (Republic of Korea).

OIE. 2014. http://www.oie.int/wahis_2/public/wahid.php/Diseaseinformation/statusdetail. Date accessed January 07, 2014.

Okell, C., Pinchbeck, G., Stringer, A., Tefera, G., and Christley, R. 2013. A community-based participatory study investigating the epidemiology and effects of rabies to livestock owners in rural Ethiopia. Preventive Veterinary Medicine, 108(1), 1-9.

Okello, A. L., Bardosh, K., Smith, J., and Welburn, S. C. 2014. One health: past successes and future challenges in three African contexts. PLoS Negl Trop Dis, 8(5), e2884.

Pieracci, E. G., Hall, A. J., Gharpure, R., Haile, A., Walelign, E., Deressa, A., . . . Ermias, B. 2016. Prioritizing zoonotic diseases in Ethiopia using a one health approach. One Health, 2, 131-135. doi:https://doi.org/10.1016/j.onehlt.2016.09.001

Riley, S., Fraser, C., Donnelly, C. A., Ghani, A. C., Abu-Raddad, L. J., Hedley, A. J., . . Thach, T. Q. 2003. Transmission dynamics of the etiological agent of SARS in Hong Kong: impact of public health interventions. Science, 300(5627), 1961-1966. 
Roth, F., Zinsstag, J., Orkhon, D., Chimed-Ochir, G., Hutton, G., Cosivi, O., . . Otte, J. 2003. Human health benefits from livestock vaccination for brucellosis: case study. Bulletin of the World Health Organization, 81(12), 867-876.

Sambo, M., Cleaveland, S., Ferguson, H., Lembo, T., Simon, C., Urassa, H., and Hampson, K. 2013. The burden of rabies in Tanzania and its impact on local communities. PLoS Negl Trop Dis, 7(11), e2510.

SEARG. 2013. http://www.searg.info/doku.php, Date accessed December 20, 2013.

Shim, E., Hampson, K., Cleaveland, S., and Galvani, A. P. 2009. Evaluating the costeffectiveness of rabies post-exposure prophylaxis: A case study in Tanzania. Vaccine, 27(51), 7167-7172. doi:10.1016/j.vaccine.2009.09.027

Smith, W., and Aerts, A. 2014. Role of contact tracing and prevention strategies in the interruption of leprosy transmission. Lepr Rev, 85(1), 2-17.

Taylor, L. H., Hampson, K., Fahrion, A., Abela-Ridder, B., and Nel, L. H. 2017. Difficulties in estimating the human burden of canine rabies. Acta Tropica, 165, 133-140. doi:https://doi.org/10.1016/j.actatropica.2015.12.007

Tenzin, Dhand, N. K., Gyeltshen, T., Firestone, S., Zangmo, C., Dema, C., . . . Ward, M. P. 2011. Dog Bites in Humans and Estimating Human Rabies Mortality in Rabies Endemic Areas of Bhutan. Plos Neglected Tropical Diseases, 5(11), e1391.

Tepsumethanon, V., Wilde, H., and Meslin, F. X. 2005. Six criteria for rabies diagnosis in living dogs. J Med Assoc Thai, 88(3), 419-422.

Thornton, P. K., Boone, R. B., Galvin, K. A., BurnSilver, S. B., Waithaka, M. M., Kuyiah, J., . .. Herrero, M. 2007. Coping Strategies in Livestock-dependent Households in East and Southern Africa: A Synthesis of Four Case Studies. Human Ecology, 35(4), 461-476. doi:10.1007/s10745-007-9118-5

Townsend, S. E., Lembo, T., Cleaveland, S., Meslin, F. X., Miranda, M. E., Putra, A. A. G., . . . Hampson, K. 2013. Surveillance guidelines for disease elimination: A case study of canine rabies. Comparative Immunology, Microbiology and Infectious Diseases, 36(3), 249-261. doi:http://doi.org/10.1016/j.cimid.2012.10.008

Welburn, S. C., Bardosh, K. L., and Coleman, P. G. 2016. Novel Financing Model for Neglected Tropical Diseases: Development Impact Bonds Applied to Sleeping Sickness and Rabies Control. Plos Neglected Tropical Diseases, 10(11), e0005000.

Wera, E., Mourits, M., Siko, M., and Hogeveen, H. 2016. Cost-Effectiveness of Mass Dog Vaccination Campaigns against Rabies in Flores Island, Indonesia. Transboundary and Emerging Diseases.

WHO. 2009. Ethiopia extends health to its people. Bulletin of the World Health Organization, 87(7), 495-496. doi:10.2471/blt.09.050709.

WHO, 2017. Human rabies: 2016 updates and call for data= Données sur la rage: mise à jour de 2016 et appel à la communication. Weekly Epidemiological Record= Relevé épidémiologique hebdomadaire, 92(7), 77-86. 
Willeberg, P., Paisley, L., and Lind, P. 2011. Epidemiological models to support animal disease surveillance activities. Rev. sci. tech. Off. int. Epiz, 30(2), 603-614.

Woolhouse, M. 2011. How to make predictions about future infectious disease risks. Philosophical Transactions of the Royal Society B: Biological Sciences, 366(1573), 2045-2054. doi:10.1098/rstb.2010.0387

Zelman, B., Melgar, M., Larson, E., Phillips, A., and Shretta, R. 2016. Global fund financing to the 34 malaria-eliminating countries under the new funding model 2014-2017: an analysis of national allocations and regional grants. Malaria Journal, 15, 118. doi:10.1186/s12936-016-1171-3

Zinsstag, J., Schelling, E., Wyss, K., \& Mahamat, M. B. 2005. Potential of cooperation between human and animal health to strengthen health systems. The Lancet, 366(9503), 2142.

Zinsstag, J., Dürr, S., Penny, M., Mindekem, R., Roth, F., Gonzalez, S. M., . . . Hattendorf, J. 2009a. Transmission dynamics and economics of rabies control in dogs and humans in an African city. Proceedings of the National Academy of Sciences, 106(35), 1499615001.

Zinsstag, J., Schelling, E., Bonfoh, B., Fooks, A. R., Kasymbekov, J., Waltner-Toews, D., and Tanner, M. 2009b. Towards a 'One Health'research and application tool box. Veterinaria Italiana, 45(1), 121-133.

Zinsstag, J., Schelling, E., Waltner-Toews, D., \& Tanner, M. 2011. From “one medicine” to "one health" and systemic approaches to health and well-being. Preventive Veterinary Medicine, 101(3), 148-156. 

Summary 
Rabies is among the oldest and most recognised diseases affecting both humans and animals. Every year, rabies is responsible worldwide for an estimated 60,000 human deaths and 9 billion USD economic losses. The economic burden is caused by premature death, direct and indirect costs of treatment, costs to the veterinary sector due to dog vaccination, and losses due to livestock deaths.

Accurate information on the incidences of rabies in humans, dogs and livestock is scarce in Ethiopia, due to the absence of proper registration systems. Based on official records, the annual human exposure rate in Ethiopia is estimated at 12 exposed human cases per 100,000 population, resulting in a death rate of 1.6 deaths per 100,000 population. The true burden of the disease is, however, expected to be higher, as a considerable proportion of rabies exposures, especially in rural communities, does not report to health centres.

Moreover, as the livestock sector in Ethiopia provides livelihoods to 65\% of the human population occurrence of rabies in cattle can also have considerable economic consequences for smallholder subsistence farmers. To date, insights on the economic impacts of livestock rabies is seriously lacking.

Domestic dogs are major sources for both human and livestock rabies in Africa and thus, mass canine vaccination is proven to be an effective means of elimination of rabies. Current canine vaccination coverages are extremely low in the cities and even non-existent in the rural communities of Ethiopia.

Over the last decade, there has been an increasing interest in Ethiopia to launch a national control programme to mitigate the impact of the disease on human and animal health, as well as to minimise the economic burden. Designing an appropriate cost-effective rabies control policy requires a clear understanding of the burden of the disease, its economic impacts, the factors associated with its burden, as well as understanding the relationship between intervention levels, the cost of intervention and potential returns.

In Chapter 1 of this study, the motivation of the study and detailed objectives were described. The overall objective of this study was to support decision-making in the control of rabies in Ethiopia by providing evidence on its health and economic burden in human as well as in cattle, and to evaluate canine vaccination strategies in Ethiopia. The broader objective was addressed through six sub-objectives. 1) to systematically assess the level of canine vaccination coverage against rabies in Africa, 2) to examine the trend of rabies incidences in African countries based on data reported to regional and international organisations through human and veterinary health authorities, 3) to estimate the incidence and economic impacts of cattle rabies in two representative livestock production systems, 4) to estimate the public health burden and postexposure treatment costs due to rabies in three representative human settlement settings, 5) to 
assess factors influencing likelihood of medical treatment-seeking and compliance to treatment regimen behaviour following rabies exposure, and 6) to evaluate cost-effectiveness of canine vaccination strategies to control rabies.

By means of an extensive literature review dog rabies vaccination coverage in Africa, in relation to dog accessibility and vaccination costs recovery schemes, was studied in Chapter 2. It showed that the majority (up to 98.1\%) of the dogs in African countries are owned and as such accessible for vaccination. Dog vaccination coverage following a 'free-of-charge", vaccination scheme was higher (68\%) than coverage under an 'owner-charged"' scheme (18\%). As most dogs in Africa are owned and therefore accessible for vaccination, a high vaccination coverage is achievable but depending on the implemented costs recovery schemes. In Chapter 3, we systematically extracted dog rabies data from reports of public health and veterinary authorities to the Southern and Eastern African Rabies Group (SEARG) and the World Organisation for Animal Health (OIE/WAHID), respectively. The reported incidence numbers from both authorities over a period of five years (2008-2012) were significantly correlated and did not indicate the presence of any trend of outbreak. However, serious inconsistencies in the reported date were observed, possibly due to the fact that human and animal health authorities report separately to the organisations involved in addition to the use of indefinite definitions of report categories set by report receiving organizations.

The burden of rabies was assessed both in cattle as well as in humans in Chapters 4-6. Chapter 4 presents estimates of the direct economic impacts of rabies at herd level in two representative subsistence cattle-farming systems in Ethiopia, namely the mixed crop-livestock and pastoral production systems. The economic impacts were assessed by means of a structured questionnaire administered to cattle-owning households selected from four districts within two administrative zones; each zone representing a cattle production system. Rabies incidence rates of $21 \%$ and $11 \%$ at herd level were calculated for the mixed crop-livestock and pastoral production systems, respectively. The incidence rate at cattle level was the same in both systems i.e. $2 \%$. Herd-level incidence rates were higher in the mixed crop-livestock system than in the pastoral system. Average economic losses per herd due to rabies were estimated at 49 USD per year for the mixed-crop livestock system, and at 52 USD per year for the pastoral system; whereas in affected herds the average losses per year were 228 USD (range 48-1016 USD) in the mixed crop-livestock system, and 477 USD (range 173-1140 USD) in the pastoral system. The average herd-level economic losses were not significantly different between the farming systems; however, once the herd was affected, the losses were significantly higher for herds in the pastoral system than for herds in the mixed crop-livestock system. As such, the losses due to rabies in cattle are relatively high for pastoral households due to their complete dependency on livestock for their livelihoods. 
In Chapter 5, we aimed at estimating the public health burden and post-exposure treatment costs of canine rabies in Ethiopia by an investigation of exposed human cases in three districts of Ethiopia representing different human settlements and agro-ecology. Annual rabid dog exposures were estimated per evaluated Bishoftu/urban, Lemuna-bilbilo/rural highland and Yabelo/rural lowland district at, respectively, 135, 101 and 86 bites, which led, respectively, to 1,4 and 3 deaths per 100,000 inhabitants. In the same district order, average costs per completed PET equalled to 23, 31 and 40 USD, which is significantly higher in the rural districts than in the urban district. A weighted extrapolation of the results to the national level indicated an annual estimate of approximately 3,000 human deaths resulting in about 93,000 disability adjusted life years (DALYs) per year and 97,000 exposed persons requiring on average 2 million USD treatment costs per year countrywide. These estimations of the burden of rabies provide decision makers insights into the potential benefits of implementing effective interventions.

Chapter 6 explored factors that determine the medical treatment-seeking behaviour following potential exposure after being bitten by a suspected dog, and the likelihood of compliance to receive sufficient doses of post-exposure prophylaxis after the visit to a health centre. An extensive survey based on case investigation was conducted on suspected rabid dog bite cases in three districts of Ethiopia. Two multivariable logistic regression models were created with a set of putative variables to explain treatment seeking and compliance outcomes. Among the putative factors tested, ownership status of the biting dog, severity of the bite, body part bitten, and distance from health centre were found to be significant predictors of the likelihood of seeking medical treatment; while household spending (which was used as a proxy for income) and distance to the health centre were found to be significant predictors of the likelihood of compliance in receiving sufficient doses of post-exposure prophylaxis. The overall likelihood of seeking medical services following rabies exposure was higher for people bitten by dogs of unknown ownership, whenever the bite was severe, when being bitten on the leg, and living at a short distance to the nearest health centre. Upon inclusion of district as an additional explanatory variable in the model, the effect of distance became less, while living in the urban district increased the likelihood of seeking treatment. In contrast, in the model to predict likelihood of compliance to treatment regimen, inclusion of district did not improve the predictive power nor the model fit. Given the insights obtained from this study, improvements in the rural districts with regard to accessibility of post-exposure prophylaxis delivering health centres in shorter distance could improve health-seeking behaviour.

Given the limited resources in developing countries, detailed cost-effectiveness analyses of canine rabies control strategies is important to support decision-making process. In this context Chapter 7 gave special attention to the evaluation of different mass vaccination strategies against rabies. Subsequently, we evaluated the cost-effectiveness of canine vaccination in two Ethiopian districts, urban and rural. We built a dynamic epidemiological model, fit to district- 
specific data on human rabies exposures and canine demography using a Bayesian Monte-Carlo Markov chain (MCMC) process. Epidemiological model output was coupled with an economic analysis to predict the human health impact (in disability-adjusted life-years (DALY)) and economic benefit (reducing human treatment costs and livestock rabies-related losses) across a range of vaccination scenarios. Human exposures, human deaths, and rabies-related livestock losses decreased consistently with increasing canine vaccination coverage. In the rural district, all vaccination scenarios were found to be cost-saving compared to the current situation (status quo) of no vaccination. In both urban and rural districts, 50\% coverage provided the greatest net health benefits at the WHO-recommended willingness-to-pay threshold over a time frame of ten years. A shorter analytical time frame led to recommendations for higher coverage in both districts, which was also the case when accounting for the threat of rabies re-introduction. Without incorporating benefits from the prevention of livestock rabies, vaccination would not have been considered to be cost-effective in rural Ethiopia, highlighting the importance of applying a broad perspective with regard to the benefit of vaccination.

Finally, in Chapter 8, we synthesised the results of this study to draw conclusions with respect to the overall objective. In this chapter we also reflected on the major findings and the methodological approaches, discussed policy implications of the results on rabies control in Ethiopia and beyond, and outlined possible research questions for future research directions.

Based on the results described in this thesis, the following main conclusions are drawn:

- In Africa, canine vaccination coverage against rabies under owner-charged scheme (18\%) is far below the free-of-charge scheme (68\%) (Chapter 2). On the other hand, most dogs in Africa are owned and accessible for vaccination against rabies if campaigns are performed at reduced costs or free-of-charge.

- Based on the available data from African regional (SEARG) and international (OIE) organisations, there is no evidence of an increasing or decreasing trend of rabies occurrence in Southern and Eastern African countries (Chapter 3).

- On average household level, rabies in cattle causes comparable economic losses (49-52 USD/year) in mixed crop-livestock and in pastoral farming systems. Losses in pastoral households (477 USD/year) are significantly higher than in mixed crop-livestock affected households (228 USD/year) signifying a serious impact as they mainly rely on their livestock for livelihoods (Chapter 4).

- Based on data collected from three representative districts in Ethiopia, the highest human rabies exposure rate is in urban district (135/100,000 inhabitants), while the health burdens are higher in rural districts (3-4 deaths per 100,000 inhabitants) than in urban (1 death per 100,000 inhabitants) districts (Chapter 5).

- Annually, rabies causes the death of about 3,000 people and leads to treatment-related expenditures of about 2 million USD nationally (Chapter 5). 
- The likelihood of seeking medical care following rabies exposure is higher in people bitten by potentially rabid dogs of unknown ownership, with severe bites, and who are living in a short distance from the nearest health centre (Chapter 6).

- The shorter the distance to the nearest health centre and the higher the income, the more likely bite victims comply with the treatment regimen (Chapter 6).

- Annual canine mass vaccination at 50\% coverage is the most cost-effective strategy to control canine rabies in urban districts as well as rural districts of Ethiopia (Chapter 7). 


\section{Acknowledgments}

The last four years resembled a long journey filled with mixed feelings of hope and frustrations. Finally, it is done! With only a limited number of pages to spend, it is not an easy task to acknowledge all the people who made this journey possible. However, I will try to do my best to extend my great appreciation to those who helped me in one way or another.

First of all, I owe special thanks to my promotor Henk, thank you for being a great mentor to me! I admire your encouragements and guidance throughout the course of this research work. Monique, you were also an incredible academic guide. Your overall advice, detailed orientation, commitment, and patience to repeatedly work on manuscripts until it gets right were few of the most important lessons. I learn a lot from your critical and meticulous scrutiny. I also extend my acknowledgment to Alfons who was willing to accept me as a student back in 2013 and later passed me to Henk. Ewaldus and Wudu were also my 'invisible advisors' as your 'old' templets saved my time that I otherwise would have spent on scratching my head.

The last chapter of this thesis could not have been completed without the guidance of Alison, Meagan, and Abhishek at Yale school of Public Health. Alison, thank you for hosting me during my visit and I really enjoyed Yale university facilities. Meagan, you were great and incredible at teaching me the techniques of modelling and R programming. I also enjoyed your hospitality during my visit to your latter home, University of Maryland in Baltimore. I really thank you very much! Crawford, I am grateful to your intellectual and overall advice throughout my study and incredible hospitality you showed me in Charlottetown of PEI. I am also grateful to the mission rabies team based in UK: Andy, Fred and Luke for sending me the dog markers which saved me lots of hardship finding the marker. Morters who sent me old hard copy publications from Cambridge University library also deserve thank for those publications of old times which I couldn't find digital version online.

I owe deep gratitude to Abraham Haile and the Zoonosis research team at the Ethiopian Public Health Institute (former Pasteur institute) who assisted me in facilitating ethical clearance and availing laboratory data. I am also thankful to the district officers and health extension workers who participated in this study with great patience, for allowing me to access local communities and availing logistic support during field data collection for the success of my research. My friends and colleagues at College of Veterinary Medicine and Agriculture, Addis Ababa University, who personally assisted me in several ways for the success of my study also deserve thank.

My pleasant and unforgettable stay at Business Economics Group (BEC) was also spiced by the company of all $\mathrm{PhD}$ students and staff members to which my special acknowledgment goes, especially to Anne, Jeanette, Claudia, Natasha, and Ilona for easing all essential administrative issues. Thank you so much for your hospitality, kindness and overall support. 
Although being away from my beloved ones for such long time was very challenging, I was also fortunate to be surrounded by friends to feel at ease. Office mates and BEC colleagues Ewaldus, Francesco, Luis, Farahnaz, Eva, Dikky, and Ethiopian fellows Wudu, Wassie, Tere, Tare,Wonde, Banchi, Mikinay, Kal, Beshir, Tade, Mekuanint, Abi, Dele, Sol, Mesi, Shime,..., and many more (not complete list) were good friends. Thank you for the great togetherness we had. I certainly think that your presence made my journey more enjoyable.

Special thanks goes to the Mahbere Kidusan community in Debre Zeit for taking care of my family especially Habte, Abe, Heni, Kiflu, Ante and their families. My parents especially Gashe, and 'Bro' Fike who always dreamed for my success deserve thank. Your constant encouragement and moral support throughout the long journey of my academic career and personal life were immense.

Finally, despite words cannot adequately express my appreciation to my best soul mate, Asni, I still would like to forward my most heartfelt gratefulness. "Enat", thank you for being such an amazing wife I could ever wish for! I would not have completed my study without your support. Each second I think of you, Afi and, Baby Sam, I felt encouraged to get this work done! and to get back home as soon as possible.

Tariku Jibat Beyene

December 18, 2017

Wageningen, the Netherlands 


\section{About the author}

Tariku Jibat Beyene was born on April 23, 1983, in Shambu, Ethiopia. He completed his primary and secondary education in Nekemte. In 2000, he joined Addis Ababa University from where he graduated with distinction as Doctor in Veterinary Medicine in 2006. Immediately after graduation, he was recruited as a lecturer at the University of Gondar where he served for a year and a half. Late in 2007 he re-joined his home university, Addis Ababa University Faculty of Veterinary Medicine, as a lecturer. Between 2010 and 2011 he followed a jointmaster's degree program hosted by FUB and AAU in International Animal Health speciality in Transboundary Animal Diseases Management. In 2013 he started a PhD study at Wageningen University and Research in the area of Epidemiology and applied (health) economics specifically on a research project entitled 'Rabies in Ethiopia: Modelling the burden and the effectiveness of control'. During his PhD tenure, he visited Yale University School of Public Health, Centre for Infectious Disease Modelling and Analysis twice as a post-graduate fellow where he was able to collaborate with renowned experts in the field of disease modelling. He also presented his findings in several international conferences and published in high impact peer reviewed journals. Besides his PhD project, Tariku has also involved in various research projects including the Global Burden of Diseases (GBD) analysis based at the Institute of Health Metrics and Evaluation in Seattle/USA, evaluation of smartphone applications for disease diagnosis and surveillance in Ethiopia in collaboration with Atlantic Veterinary College in Canada, and several local projects.

Tariku's research interest focuses on the integration of epidemiological, economic, and social aspects of disease control and prevention through providing practical and feasible tools that support decision-makers in the efficient allocation of resources. For more information, he can be reached at jibattariku@gmail.com. 


\section{Scientific publications from this thesis}

1. Tariku Jibat, Monique C. M. Mourits, Henk Hogeveen (2016). Incidence and Economic impact of rabies in cattle population in Ethiopia. Preventive Veterinary Medicine. 130 (2016) 67-76. http://dx.doi.org/doi:10.1016/j.prevetmed.2016.06.005.

2. Tariku Jibat, Henk Hogeveen. Monique C.M. Mourits (2015). Systematic review and metaanalysis on dog rabies vaccination coverage in Africa: A question of dog accessibility or cost recovery? PLoS Negl Trop Dis 9(2): e0003447. http://dx.doi.org/10.1371/journal.pntd.0003447

3. Tariku Jibat Beyene, Monique C. M. Mourits, Henk Hogeveen (2016). Dog rabies data reported to multinational organizations from Southern and Eastern African Countries. BMC Research Notes. 2017 10:199. https://doi.org/10.1186/s13104-017-2527-7

4. Tariku Jibat Beyene, Monique C. M. Mourits, Abraham Haile, Henk Hogeveen. Estimating the burden of rabies in Ethiopia by dog bite victims tracing (Under review by PLOS ONE journal).

5. Tariku Jibat Beyene, Monique C. M. Mourits, Crawford W Revie, Henk Hogeveen. Determinants of health seeking behavior following rabies exposure in Ethiopia (Under review by Zoonoses and public health journal).

\section{Contributed to Conferences and seminars}

1. Tariku Jibat Beyene, Monique C.M. Mourits, Abraham Haile Kidane, Henk Hogeveen. Public health burden of Rabies in Ethiopia' SVEPM Conference, Inverness, UK. March 20-31, 2017.

2. Tariku Jibat, Monique C. M. Mourits, Tassew Woldehanna, Henk Hogeveen. Impact assessment of livestock rabies in Ethiopia. Dutch Society for Veterinary Epidemiology \& Economics, Deventer, The Netherlands, October 2nd, 2015.

2. Tariku Jibat. A One health framework for quantifying burden and evaluating the economics of NTD control: Case of Rabies in Ethiopia. ARNDT conference, Blantyre, Malawi. November 17-19, 2015.

4. Tariku Jibat Beyene, Monique C. M. Mourits, Crawford W. Revie, Henk Hogeveen Determinants of health seeking behaviors following rabies exposure in Ethiopia. WASS multidisciplinary seminar (Ph.D. Day), May 18, 2017. 
Tariku Jibat Beyene

Wageningen School of Social Sciences (WASS)

Completed Training and Supervision Plan

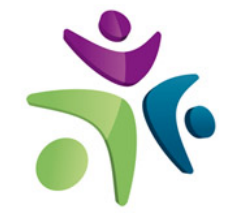

Wageningen School

of Social Sciences

\begin{tabular}{|c|c|c|c|}
\hline Name of the learning activity & Department/Institute & Year & ECTS* \\
\hline \multicolumn{4}{|l|}{ A) Project related competences } \\
\hline $\begin{array}{l}\text { Modern Statistics for the Life Sciences, ABG- } \\
30806\end{array}$ & WUR & 2014 & 6.0 \\
\hline $\begin{array}{l}\text { Applied Economic modelling for Veterinary } \\
\text { Science }\end{array}$ & $\begin{array}{l}\text { Graduate School of Life } \\
\text { Sciences, Utrecht University }\end{array}$ & 2014 & 3.0 \\
\hline Mathematical modelling of infectious diseases & $\begin{array}{l}\text { Graduate School of Life } \\
\text { Sciences, Utrecht University }\end{array}$ & 2014 & 3.0 \\
\hline $\begin{array}{l}\text { Economics of animal health and food safety, } \\
\text { BEC-52806 }\end{array}$ & WUR & 2014 & 6.0 \\
\hline Introduction to R for statistical analysis & PE\&RC & 2014 & 0.6 \\
\hline Bayesian statistics & PE\&RC & 2015 & 0.6 \\
\hline \multicolumn{4}{|l|}{ B) General research related competences } \\
\hline WASS Introduction course & WASS & 2013 & 1.0 \\
\hline Writing PhD proposal & WUR & 2013 & 6.0 \\
\hline Systematic approaches to reviewing literature & WASS & 2013 & 3.7 \\
\hline BEC PhD meetings & WUR & 2013-2017 & 2.0 \\
\hline $\begin{array}{l}\text { 'A One health framework for quantifying burden } \\
\text { and evaluating the economics of NTD control: } \\
\text { Case of Rabies in Ethiopia' }\end{array}$ & $\begin{array}{l}\text { The ARNTD } 3^{\text {nd }} \text {, Blantyre, } \\
\text { Malawi }\end{array}$ & 2015 & 1.0 \\
\hline $\begin{array}{l}\text { 'Impact assessment of rabies in livestock in } \\
\text { Ethiopia' }\end{array}$ & $\begin{array}{l}27^{\text {th }} \text { VEEC, Deventer, The } \\
\text { Netherlands }\end{array}$ & 2015 & 1.0 \\
\hline $\begin{array}{l}\text { 'Public health burden and exposure costs of } \\
\text { rabies in Ethiopia' }\end{array}$ & $\begin{array}{l}\text { SVEPM, Inverness, United } \\
\text { Kingdom }\end{array}$ & 2017 & 1.0 \\
\hline $\begin{array}{l}\text { 'Determinants of health seeking behaviour } \\
\text { following rabies exposure in Ethiopia' }\end{array}$ & $\begin{array}{l}\text { WASS PhD day, Wageningen } \\
\text { The Netherlands }\end{array}$ & 2017 & 1.0 \\
\hline \multicolumn{4}{|c|}{ C) Career related competences/personal development } \\
\hline WASS PhD council membership & WASS & 2013-2014 & 2.0 \\
\hline $\begin{array}{l}\text { Information Literacy including EndNote } \\
\text { Introduction }\end{array}$ & WGS & 2013 & 0.6 \\
\hline $\begin{array}{l}\text { Techniques for Writing and Presenting Scientific } \\
\text { Papers }\end{array}$ & WGS & 2014 & 1.2 \\
\hline Reviewing a Scientific Paper & WGS & 2017 & 0.1 \\
\hline $\begin{array}{l}\text { Quantitative data analysis: multivariate } \\
\text { techniques, YRM-60306 }\end{array}$ & WUR & 2013 & \\
\hline $\begin{array}{l}\text { Quantitative Veterinary Epidemiology, QVE- } \\
30306\end{array}$ & WUR & 2014 & \\
\hline
\end{tabular}

Total

* One credit according to ECTS is on average equivalent to 28 hours of study load

${ }^{1} \mathrm{BEC}=$ Business Economics Group; WUR= Wageningen University and Research centre; SVEPM= Society for Veterinary Epidemiology and Preventive Medicine; WASS= Wageningen School of Social Sciences; PE \& RC= Graduate School for Production Ecology and Resource Conservation; ARNTD= African Researchers Network for Neglected Tropical Diseases; WGS= Wageningen Graduate Schools, VEEC= Dutch Society for Veterinary Epidemiology \& Economics 
The research described in this thesis was funded by The Netherlands Organization for International Cooperation in Higher Education (NUFFIC).

Lay Out, Published and Printed by: Proefschriftmaken.nl 